\title{
Economic impact and inequalities in diabetes in South Africa
}

Citation for published version (APA):

Mutyambizi, C. (2020). Economic impact and inequalities in diabetes in South Africa. [Doctoral Thesis, Maastricht University]. Gildeprint en Universitaire Pers Maastricht.

https://doi.org/10.26481/dis.20200706cm

Document status and date:

Published: 01/01/2020

DOI:

$10.26481 /$ dis. $20200706 \mathrm{~cm}$

Document Version:

Publisher's PDF, also known as Version of record

\section{Please check the document version of this publication:}

- A submitted manuscript is the version of the article upon submission and before peer-review. There can be important differences between the submitted version and the official published version of record.

People interested in the research are advised to contact the author for the final version of the publication, or visit the DOI to the publisher's website.

- The final author version and the galley proof are versions of the publication after peer review.

- The final published version features the final layout of the paper including the volume, issue and page numbers.

Link to publication

\footnotetext{
General rights rights.

- You may freely distribute the URL identifying the publication in the public portal. please follow below link for the End User Agreement:

www.umlib.nl/taverne-license

Take down policy

If you believe that this document breaches copyright please contact us at:

repository@maastrichtuniversity.nl

providing details and we will investigate your claim.
}

Copyright and moral rights for the publications made accessible in the public portal are retained by the authors and/or other copyright owners and it is a condition of accessing publications that users recognise and abide by the legal requirements associated with these

- Users may download and print one copy of any publication from the public portal for the purpose of private study or research.

- You may not further distribute the material or use it for any profit-making activity or commercial gain

If the publication is distributed under the terms of Article $25 \mathrm{fa}$ of the Dutch Copyright Act, indicated by the "Taverne" license above, 


\section{Economic impact and inequalities in diabetes in South Africa}

\section{Citation for published version (APA):}

Mutyambizi, C. (2020). Economic impact and inequalities in diabetes in South Africa. Maastricht University. https://doi.org/10.26481/dis.20200706cm

Document status and date:

Published: 01/01/2020

DOI:

$10.26481 /$ dis. $20200706 \mathrm{~cm}$

Document Version:

Publisher's PDF, also known as Version of record

\section{Please check the document version of this publication:}

- A submitted manuscript is the version of the article upon submission and before peer-review. There can be important differences between the submitted version and the official published version of record.

People interested in the research are advised to contact the author for the final version of the publication, or visit the DOI to the publisher's website.

- The final author version and the galley proof are versions of the publication after peer review.

- The final published version features the final layout of the paper including the volume, issue and page numbers.

Link to publication

\footnotetext{
General rights rights.

- You may freely distribute the URL identifying the publication in the public portal. please follow below link for the End User Agreement:

www.umlib.nl/taverne-license

Take down policy

If you believe that this document breaches copyright please contact us at:

repository@maastrichtuniversity.nl

providing details and we will investigate your claim.
}

Copyright and moral rights for the publications made accessible in the public portal are retained by the authors and/or other copyright owners and it is a condition of accessing publications that users recognise and abide by the legal requirements associated with these

- Users may download and print one copy of any publication from the public portal for the purpose of private study or research.

- You may not further distribute the material or use it for any profit-making activity or commercial gain

If the publication is distributed under the terms of Article 25fa of the Dutch Copyright Act, indicated by the "Taverne" license above, 
Economic impact and inequalities in diabetes in South Africa

Chipo Mutyambizi 
Economic impact and inequalities in diabetes in South Africa (C) 2020, Chipo Mutyambizi

ISBN: 9789464023732

Cover design by: Tendai Mutyambizi

Published by Gildeprint

All rights reserved. No part of this publication may be reproduced, stored in a retrieval system, or transmitted in any form or by any means, electronic, mechanical, photocopying, recording or otherwise, without the written permission from the author. 


\title{
Economic impact and inequalities in diabetes in South Africa
}

\author{
DISSERTATION
}

To obtain the degree of Doctor at Maastricht University on the authority of the Rector Magnificus, Prof. Dr. Rianne M. Letschert in accordance with the decision of the Board of Deans, to be defended in public on Monday, 6 July, 2020, at $10.00 \mathrm{hrs}$ 
Promoters:

Prof. Dr. Wim Groot

Prof. Dr. Milena Pavlova

Assessment Committee:

Prof. Dr. Henriette Maassen van den Brink, Maastricht University (Chair)

Prof. Dr. Hein de Vries, Maastricht University

Prof. Dr. Carmen Dirksen, Maastricht University

Prof. Dr. Susan Cleary, University of Cape Town

Prof. Dr. Andre P Kengne, South African Medical Research Council

The research presented in this thesis was conducted at CAPHRI Care and Public Health Research Institute, Department Health Services Research of Maastricht University. CAPHRI participates in the Netherlands School of Public Health and Care Research CaRe. 
Table of Contents

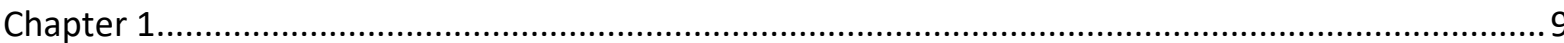

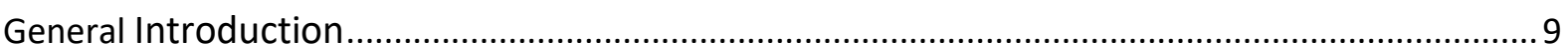

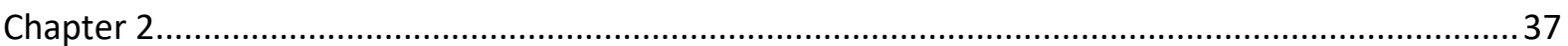

Cost of diabetes mellitus in Africa: a systematic review of existing literature .....................................37

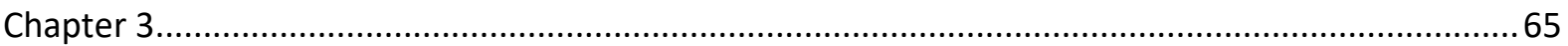

Incidence, socio-economic inequalities and determinants of catastrophic health expenditure and impoverishment for diabetes care in South Africa: a study at two public hospitals in Tshwane..........65

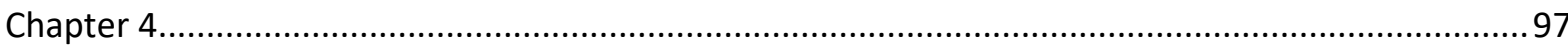

Lifestyle and socio-economic inequalities in diabetes prevalence in South Africa: A decomposition analysis

Chapter 5

Inequalities and factors associated with adherence to diabetes self-care practices amongst patients

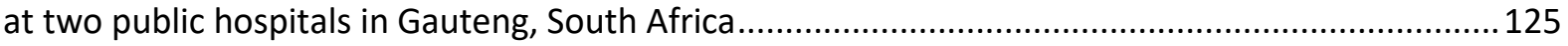

Chapter 6

Barriers and enablers of diabetes self-care - a qualitative study of people with diabetes in South

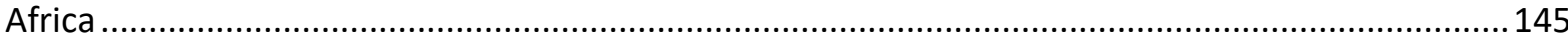

Chapter 7

The extent and determinants of diabetes and cardiovascular disease comorbidity in South Africa results from the South African National Health and Nutrition Examination Survey (SANHANES-1).. 169

Chapter 8

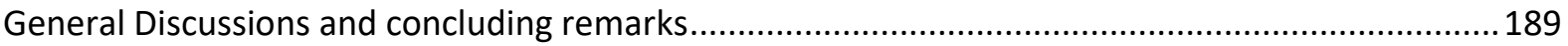

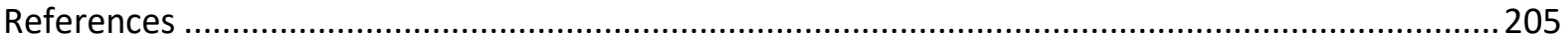

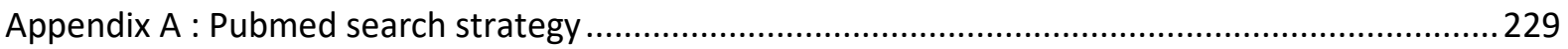

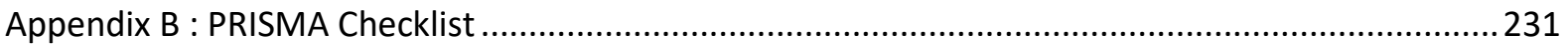

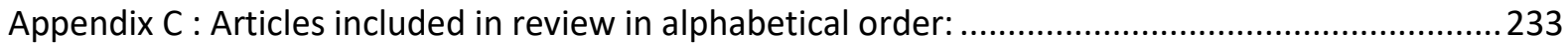

Appendix D : Cost ratio for individuals with and without diabetes mellitus complications...............237

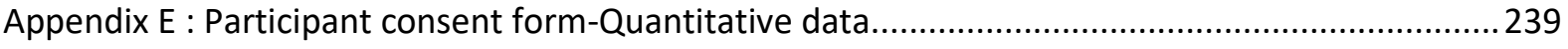

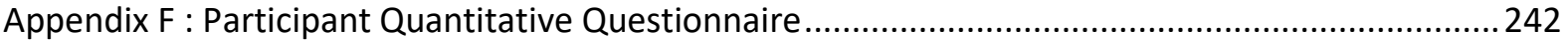

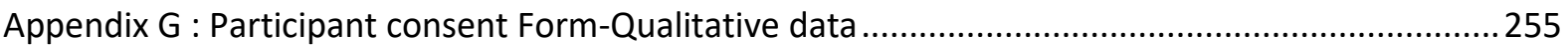

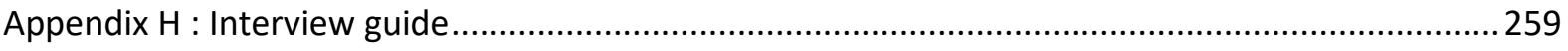

Appendix I : COREQ (COnsolidated criteria for REporting Qualitative research) Checklist ................263

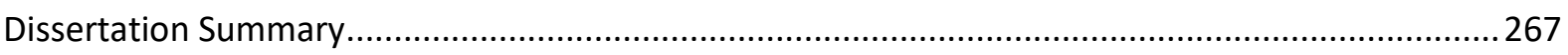

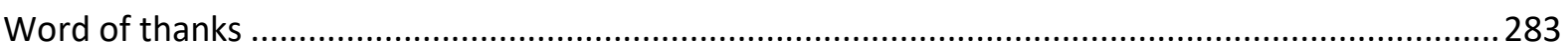

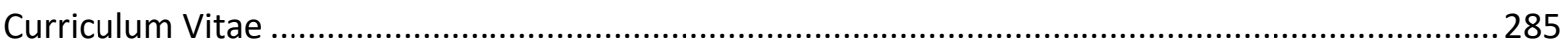

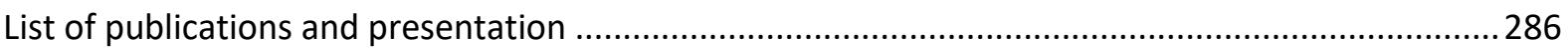




\section{List of Tables}

Table 2-1 General characteristics of studies included in the review 47

Table 2-2 Components of direct and indirect costs for diabetes mellitus and reflecting cost.............52

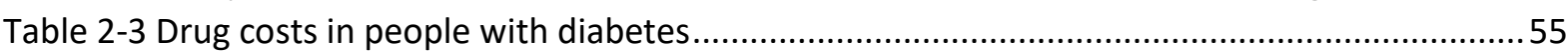

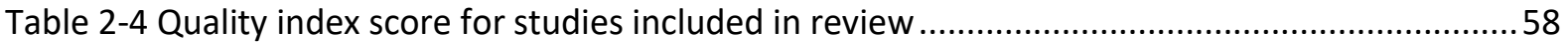

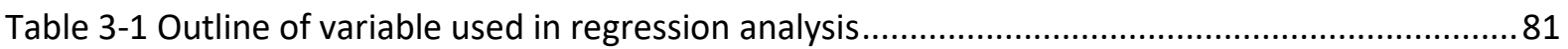

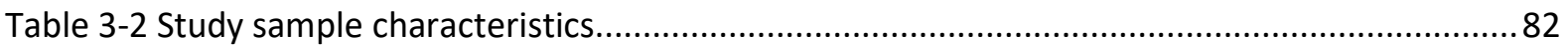

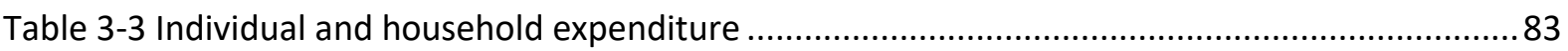

Table 3-4 Catastrophic health expenditure and impoverishment related to diabetes care (\%) ...........86

Table 3-5 Catastrophic health expenditure and impoverishment related to diabetes care, by wealth

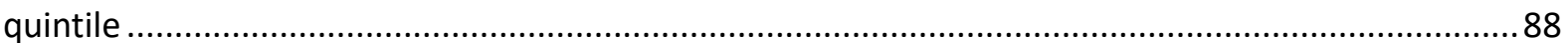

Table 3-6 Concentration indices for catastrophic health expenditure for diabetes care....................89

Table 3-7 Factors associated with catastrophic health expenditure for diabetes care ......................... 91

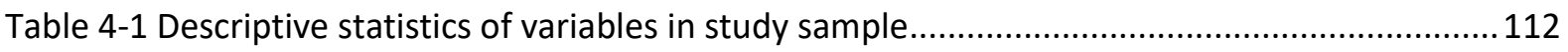

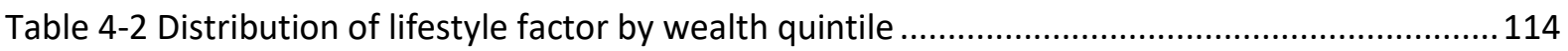

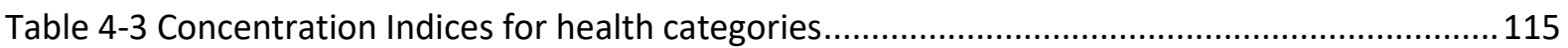

Table 4-4 Decomposition of inequality in self-reported diabetes and total diabetes.......................116

Table 5-1 Socio-demographic characteristics of people with diabetes $(\mathrm{N}=396)$................................134

Table 5-2 Diabetes self-care behaviours amongst people with diabetes..........................................135

Table 5-3 Distribution of self-care behaviour by wealth quintile .................................................136

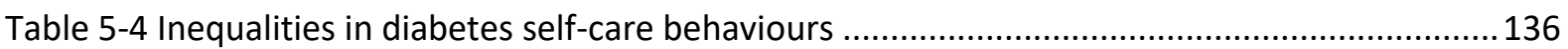

Table 5-5 Factors associated with diabetes self-care behaviours (multivariable logistic regressions)

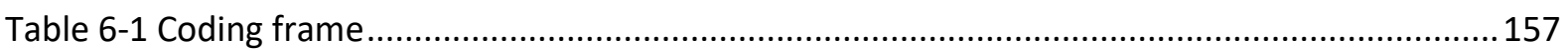

Table 7-1. Prevalence of self-reported diabetes and diabetic comorbidity ( $\mathrm{N}=12594)$....................178

Table 7-2 Logistic regression analyses of factors associated with diabetes without CVD comorbidity,

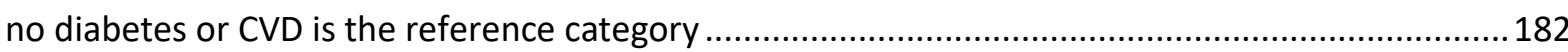

Table 7-3 Logistic regression analyses of factors associated with diabetes-CVD comorbidity, no

diabetes or CVD is the reference category 


\section{List of Figures}

Figure 1-1 Total number of adults living with diabetes (20-79 years old) ......................................13

Figure 1-2 Total health care expenditure by people with diabetes (20-79 years) (Billion USD) ..........16

Figure 1-3 Commission on the Social Determinants of Health conceptual framework ......................20

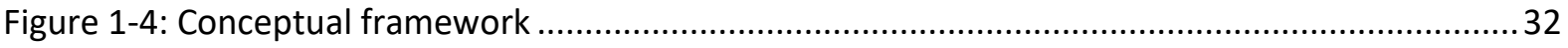

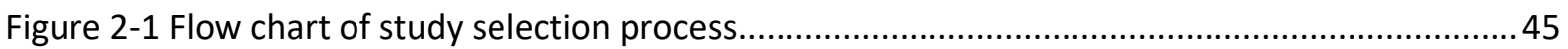

Figure 3-1 Mean of OOP health expenditure for diabetes care by socio-economic status................. 84

Figure 4-1 Flow chart for selecting the analytical sample from the general survey sample .............103

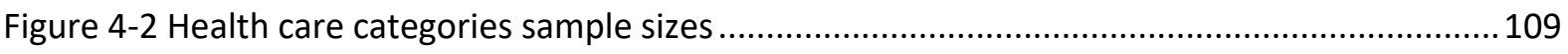

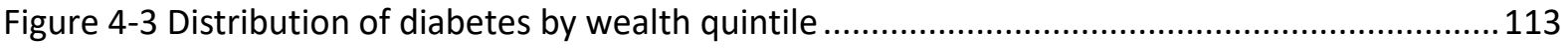

Figure 7-1 Cardiovascular comorbid conditions in people with diabetes .......................................179

Figure 7-2 Prevalence of self-reported cardiovascular comorbidities among people with diabetes

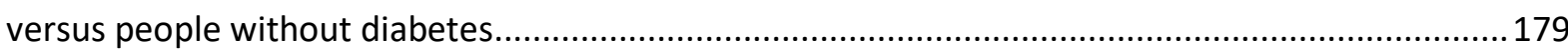




\section{Abbreviations}

ADA

BMI

$\mathrm{CC}$

$\mathrm{Cl}$

$\mathrm{COI}$

$\mathrm{CSDH}$

CVD

DDS

EA

GDP

HSRC

IDF

LMICs

MET

NCD

OOP

SANHANES-1

SDG

SEMDSA

SES

T1DM

T2DM

TTI

UPFS

USD

WHO
American Diabetes Association

Body Mass Index

Concentration Curve

Concentration Index

Cost of Illness

Commission on Social Determinants of Health

Cardiovascular disease

Dietary Diversity Score

Enumeration area

Gross Domestic Product

Human Sciences Research Council

International Diabetes Federation

Low- and Middle-Income Countries

Metabolic Equivalents

Non-Communicable Diseases

Out of pocket

South African National Health and Nutrition Examination Survey

Sustainable Development Goals

Society for Endocrinology, Metabolism and Diabetes of South Africa

Socio-economic Status

Type 1 Diabetes Mellitus

Type 2 Diabetes Mellitus

Theory of triadic influence

Uniform Payment Fee Schedule

United States Dollar

World Health Organisation 
Chapter 1

General Introduction 


\subsection{Scope of the dissertation}

If no measures are taken, a continued rise in diabetes is eminent and will have tremendous effects on health care systems, societies and economies at large (International Diabetes Federation [IDF], 2017; World Health Organisation [WHO], 2016a). As a result, global agendas such as the WHO Global Plan for the Prevention and Control of Non-Communicable Diseases (NCDs) 2013-2020 (WHO, 2013) and the Sustainable Development Goals (SDGs) targeted for 2030 (United Nations General Assembly, 2015) have turned their focus to the prevention and management of diabetes. Commonly referred to as the hidden scourge or the $21^{\text {st }}$ century challenge, chronic illnesses, such as diabetes, are becoming more common in developing countries and poorer societies (IDF, 2017).

Diabetes is a chronic illness that is better managed when diagnosed early. Management of diabetes also improves with self-care activities including frequent hospital check-ups (WHO, 2016a). Unfortunately, in many settings, particularly in low- and middle-income countries (LMICS), the resources necessary for the diagnosis, treatment and management of diabetes are not available, and in cases where they are, they remain out of reach for the marginalised poor individuals (Kengne, Mchiza, Amoah, \& Mbanya, 2013). Once diagnosed, people with diabetes require frequent medical check-ups (WHO, 2016a). In countries that do not offer adequate financial protection, this may mean that households with people with diabetes are at risk of being subjected to experiencing catastrophic health expenditure and ultimately impoverishment (IDF, 2017). Furthermore, the lack of effective policies that promote the adoption of healthy lifestyles necessary for the prevention and management of diabetes, means that abatement of the disease will not be achieved. This has dire consequences on individuals, households and economies. When diabetes is uncontrolled this negatively affects well-being and often leads to adverse health outcomes such as the development of diabetic complications and mortality (NCD Risk Factor Collaboration, 2016).

Whilst data from high-income countries show that diabetes is more concentrated amongst the poor, the few data from LMICs show diabetes is frequently higher amongst the rich (Biswas, Islam, Linton, \& Rawal, 2016; De Silva et al., 2018; Moradi et al., 2016). Nevertheless, evidence suggests that this trend is changing in some middle-income countries (WHO, 2016a). 
In an effort to address the rising prevalence of diabetes and improve diabetes care, it is important to advance our understanding of the economic impact and inequalities in various aspects of diabetes prevalence, cost, treatment, management and outcomes.

This dissertation looks at the costs and economic impact of diabetes in South Africa and seeks to assess the inequalities in the economic impact of diabetes and estimate inequalities across the multiple facets of diabetes prevalence and self-care management. Research on this topic is important for various reasons. The prevalence of diabetes is increasing and will place pressure on the health care system as the demand for health care services by people with diabetes increases. Given that the South African health care system is faced with many challenges, the upsurge in demand will place pressure on already strained public health care resources that support over $80 \%$ of the country's population (National Department of Health, 2011a). The rising prevalence of diabetes mainly affects the working age population. This has the potential to severely impact the livelihood of families and economic productivity. Furthermore, in South Africa, the prevailing income inequalities (Hurlbut, 2018) are accompanied by a disproportionate distribution of health across socio-economic groups (Elgar, 2010). In the case of diabetes, these socio-economic inequalities not only result in a disproportionate distribution of diabetes by socio-economic status but also to a disproportionate distribution of diabetes self-care practices which in turn impacts diabetes health outcomes.

In addition to providing new empirical evidence on the economic impact of and inequalities in diabetes, this dissertation also has policy relevance. Firstly, there is consensus amongst policy makers globally on the need to protect patients against catastrophic expenditure or impoverishment due to health care. This is evident in the promotion of National Health Insurance by various global and national institutional bodies (WHO and the World Bank, 2017). Furthermore, policy makers recognise the importance of diabetes management via the adoption of recommended self-care practices. The WHO Global Action Plan for the prevention and control of NCDs argues for a multi-sectoral approach at national, regional and global levels in the prevention and control of NCDs. By exploring the costs of diabetes in South Africa and establishing the inequalities in various aspects of diabetes, this dissertation seeks to provide evidence that can help policymakers to make informed decisions in the achievement 
of the targets set out in the SDGs and achievement of NHI in South Africa. South Africa will soon be implementing a $\mathrm{NHI}$, but there is limited information on the economic impact of diabetes on households to fully formulate mitigating measures and adequate social safety nets. Demands on the country's public finances call for prudent allocation of finances and well-planned cost-effective health interventions.

\subsection{The growing burden of diabetes}

This section provides an overview of the various aspects of diabetes; various types of diabetes, the prevalence, mortality and associated risk factors of diabetes, economic impact of diabetes and diabetes management.

\subsubsection{Diabetes mellitus: a definition}

Diabetes is described as a state of elevated blood glucose levels (hyperglycaemia), which is a result of the pancreas not producing enough insulin or when cells cannot use the insulin the body produces (Amod, 2012; IDF, 2001; WHO, 2016a). There are three main types of diabetes:

- Type 1 - is commonly referred to as insulin dependent diabetes and occurs when the body fails to produce insulin.

- Type 2 - is also called adult onset diabetes and results from a variable combination of inadequate insulin production and inability of the body to effectively use insulin. Globally this is the most common type of diabetes.

- Gestational diabetes - temporarily occurs in pregnant women who have never had diabetes before. This type of diabetes increases the risk of developing type 2 diabetes.

Because gestational diabetes is only a temporary illness, the scope of this dissertation covers individuals with type 1 and or type 2 diabetes.

\subsubsection{Diabetes indicators: prevalence, mortality and associated risk factors}

Diabetes is an NCD, which is fast becoming a world pandemic. Whilst global trends indicate a rise in the prevalence of diabetes, faster increases are evident in LMICs compared to high- 
income countries (Guariguata et al., 2014; NCD Risk Factor Collaboration, 2016). These regional differences are likely the result of higher increases in adiposity, differences in genetic susceptibility, lifestyle behaviours (e.g. physical activity) and health care system resources (NCD Risk Factor Collaboration, 2016). The disease, no longer associated with affluence, is common across all socio-economic groups (IDF, 2017).

The rise in the prevalence of diabetes is evident in Figure 1-1. Globally, the number of people between the age group of 20-79 years with diabetes, is projected to increase from 463 million in 2019 to 700 million in 2045 (IDF, 2019).

Figure 1-1 Total number of adults living with diabetes (20-79 years old)

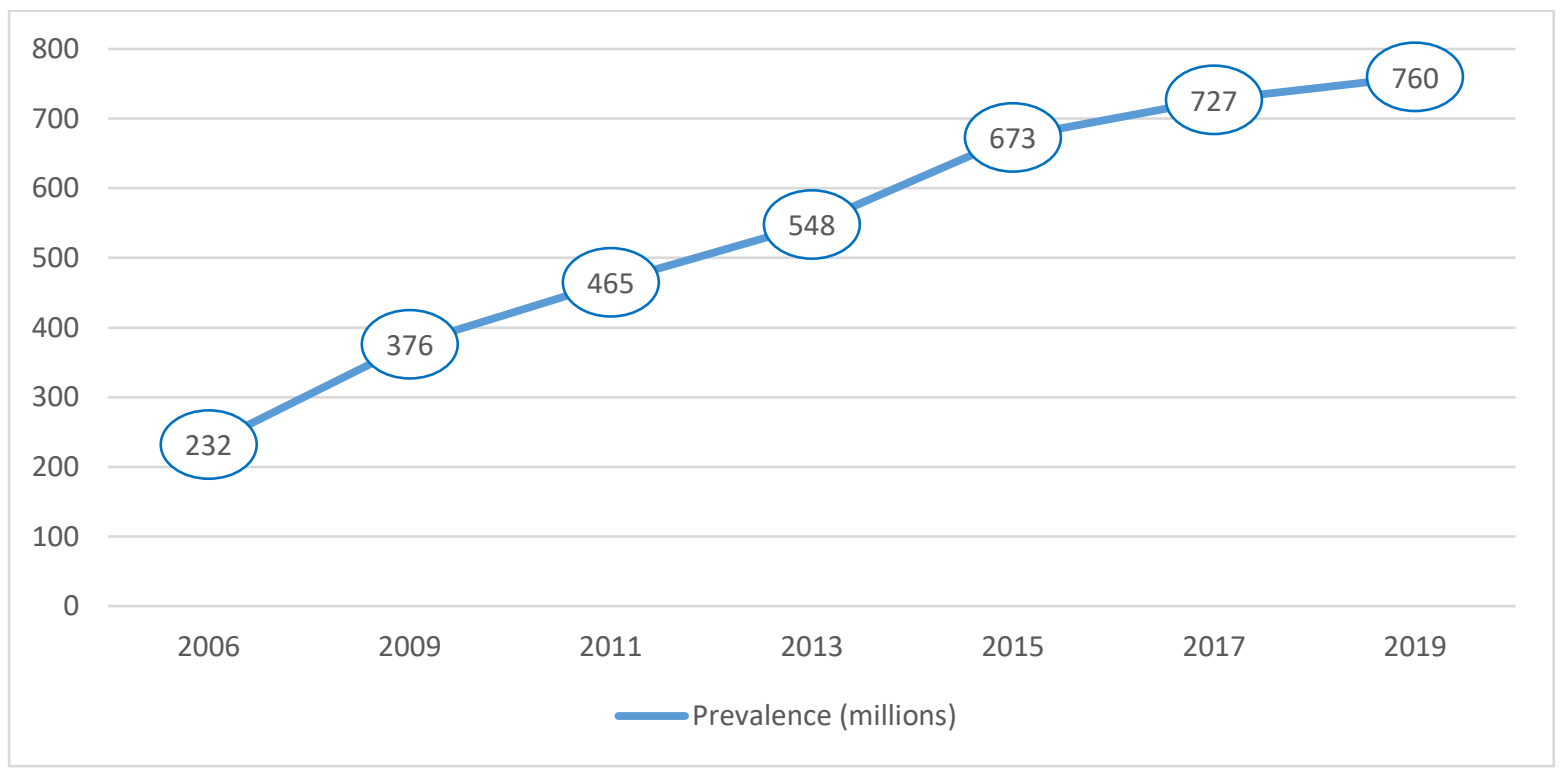

Source: Adapted from IDF, IDF Atlas, $9^{\text {th }}$ edition (IDF, 2019)

In South Africa, the prevalence of diabetes was estimated at 8.3\% in 2013 (approximately 2.6 million within the 20-79 year age group) (Guariguata et al., 2014). This prevalence is expected to increase to $9.9 \%$ in 2035 (approximately 3.8 million within the $20-79$ year age group) (Guariguata et al., 2014). Demographic and provincial variations with regards to the prevalence of diabetes in South Africa, have been reported in previous studies. A study by Stokes et al. finds that the prevalence of diabetes between the years 2011-2012 was higher amongst those of Indian descent (Stokes et al., 2017). The study also shows that the proportion of unscreened people living with diabetes is higher amongst Africans when compared to other races. Health Systems Trust provincial estimations based on the National 
Income Dynamics Study, show a prevalence of diabetes that varies from as high as $17.8 \%$ in the Western Cape to $6.9 \%$ in Mpumalanga (Massyn et al., 2019). When examined across different sub-groups of the South African population, the highest prevalence of people living with diabetes is amongst those older than 75 years, amongst females and those residing in urban formal areas (Stokes et al., 2017).

Diabetes is one of the leading causes of years-of-life lost globally (GBD 2015 Mortality and Causes of Death Collaborators, 2016). It is within the top ten leading causes of death and is estimated that within the 20-79 year age group, the number of deaths due to diabetes is 4 million globally (IDF, 2017). In South Africa, diabetes was the second biggest underlying cause of death in 2016 and the biggest underlying cause of death for both the Indian and coloured population groups (Statistics South Africa, 2018c). Amongst all NCDs, diabetes together with cancer, cardiovascular diseases (CVDs) and respiratory diseases account for over $80 \%$ of deaths due to NCDs. The increased mortality and morbidity of diabetes strikes mostly people within their productive years, reducing their economic productivity and impoverishing families. In LMICs, the percentage of deaths that occur prior to the age of 70 years, is higher than in high-income countries (WHO, 2016a). The burden of diabetes is amplified by the fact that globally, $50 \%$ of all people with diabetes are not diagnosed and hence not undergoing treatment (IDF, 2017). In South Africa, it is estimated that close to half of individuals with diabetes are undiagnosed (Stokes et al., 2017). Late diagnosis can be fatal and is often associated with the development of diabetic complications.

Along with the increases in the prevalence of diabetes as a single disease, there is increasing evidence that people with diabetes are at an increased risk of acute complications (such as seizures and diabetic commas) or chronic illnesses (such as heart disease and kidney disease) (Deshpande, Harris-Hayes, \& Schootman, 2008; IDF, 2017). People with diabetes are reportedly twice as likely to develop another CVD compared to people not living with diabetes (Emerging Risk Factors Collaboration, 2010; IDF, 2001) and are therefore at an increased risk of heart attack, stroke and limb amputations (IDF, 2001). Furthermore, people with diabetes and hypertension suffer from stroke twice as often as people with hypertension alone (IDF, 2001). Compared with people without diabetes, people with diabetes have a 2 to 3 times 
greater risk of heart failure, 15 to 50 -fold risk of lower-limb amputation and experience transient ischaemic attacks 2 to 6 times more frequently (IDF, 2001).

CVDs and renal diseases are the main cause of death in diabetes globally (IDF, 2017). Chronic kidney disease due to diabetes resulted in 418000 deaths globally in 2015 (GBD 2015 Mortality and Causes of Death Collaborators, 2016) whilst in high-income countries approximately 325000 vascular deaths per year have been attributed to diabetes (Emerging Risk Factors Collaboration, 2010). These mortality rates will continue to rise if the prevalence of diabetes continues to increase. These complications increase the complexity of the disease burden, directly worsening the health outcomes and accounting for the high mortality of diabetes (IDF, 2001). Furthermore, diabetes complications increase the costs related to inpatient and out-patient hospital visits, placing an increased strain on health care systems, individuals and households.

\subsubsection{Economic impact}

Several studies have examined the effects of NCDs on health care systems, economies, households and individuals. It is estimated that for every $10 \%$ rise in mortality due to NCDs, the annual economic growth reduces by $0.5 \%$ (Beaglehole et al., 2011). A literature review undertaken in 2006 revealed that the cost of chronic diseases ranges from $0.02 \%$ to $6.77 \%$ of a country's Gross Domestic Product (GDP) (Suhrcke, Nugent, Stuckler, \& Rocco, 2006). In many countries, it is estimated that diabetes consumes up to $10 \%$ of national health care budgets (IDF, 2001). Close to half of these costs is a result of diabetic complications (IDF, 2001).

The costs associated with diabetes include the direct costs such as those related to health care use, caregiver costs, travel costs and indirect costs such as productivity losses, work absenteeism and caregiver productivity losses (Bommer et al., 2018). The IDF estimates that total health care expenditure due to diabetes in 2019 will come up to US\$760 billion globally for the 20-79 years age group. This figure grew from a total of US\$232 billion in 2006 (see Figure 1-2) and is predicted to increase to US\$845 billion in 2045 (IDF, 2019). The burden of diabetes is disproportionately higher in LMICs when compared to high-income countries 
because those affected with diabetes in high-income countries are mostly over the age of 50 years and those affected most in LMICs are below the age of 50 years (i.e. the working age population) (Guariguata et al., 2014). The IDF forecasts that the second largest increase in diabetes expenditure between 2017 and 2045 will be experienced within the African region (IDF, 2017). The burden of diabetes on patients and families is intensified by the common use of out of pocket (OOP) payments for health care in African countries (Mapa-Tassou, Katte, Maadjhou, \& Mbanya, 2019; Smith-Spangler, Bhattacharya, \& Goldhaber-Fiebert, 2012). In the IDF African region, South Africa is one of the three countries with the largest average health expenditure on diabetes (IDF, 2017). A study by Erzse et al. (2019) estimated that the total public sector health care costs in 2018 due to diagnosed type 2 diabetes were R2.7 billion (United States Dollar [USD] 198 million) (Erzse et al., 2019). Ncube-Zulu and Danckwerts (2014) estimates that in 2009 the average hospitalisation costs for a patient with diabetes was approximately R27 216 (USD 6 253) whilst that for someone without diabetes was R 18 185 (USD 4 178) (Ncube-Zulu \& Danckwerts, 2014).

Figure 1-2 Total health care expenditure by people with diabetes (20-79 years) (Billion USD)

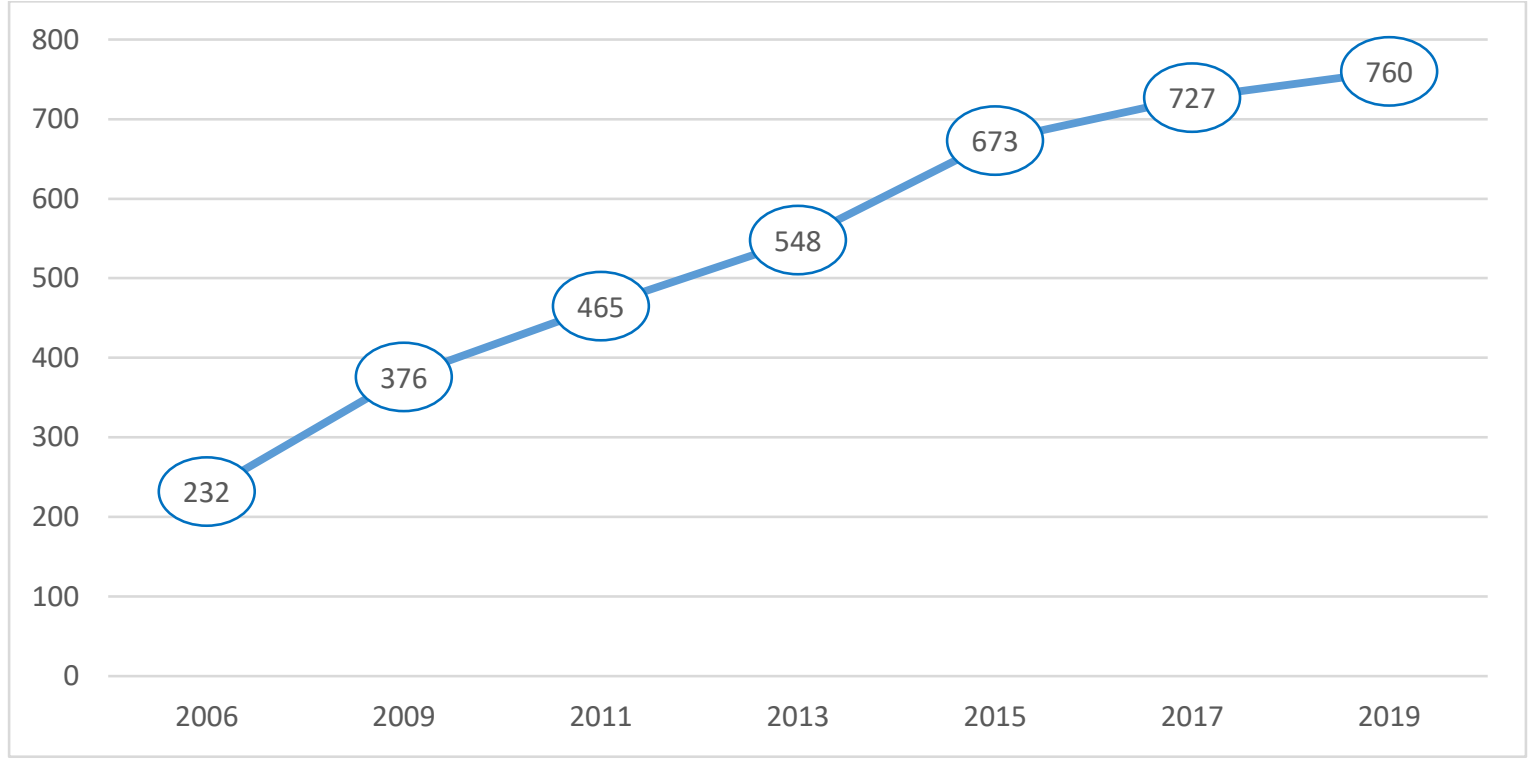

Source: Adapted from IDF, IDF Atlas, $9^{\text {th }}$ edition (IDF, 2019)

It is clear that the continued rise in the prevalence of diabetes could have enormous implications in economic terms. Reducing the rising tide of morbidity and mortality due to diabetes includes preventing diabetes itself through an adoption of healthier lifestyle by people not living with diabetes and where diabetes is present, an adoption of and adherence 
to recommended diabetes self-care practices in order to prevent or delay the onset of diabetic complications.

\subsubsection{Self-care in diabetes management}

Diabetes is a complex and progressive disease which can contribute to the damage and failure of various organs such as the heart, kidneys and nerves if glucose levels are not adequately managed (WHO, 2016a). In order to reduce the mortality, morbidity and health care costs associated with diabetes, focus needs to be placed on diabetes management. Diabetes control can be achieved through the use of diabetic medications, increasing diabetes awareness and knowledge, the adoption of healthy lifestyles (for example exercising, healthy diet) and regular access to health care for regular screening of diabetic complications (IDF, 2017; WHO, 2016a).

Diabetes care involves continuous management actions with over $95 \%$ of this care being done by the people with diabetes themselves (Funnell \& Anderson, 2000). Diabetes self-care is defined as a lifelong commitment to perform day to day behavioural routines that assist in glucose control, and avoid the occurrence of health complications (Clement, 1995; Shrivastava, Shrivastava, \& Ramasamy, 2013). Clinical guidelines such as those by the American Diabetes Association (ADA) and the Society for Endocrinology, Metabolism and Diabetes of South Africa (SEMDSA) recommend that people with diabetes must adhere to self-care behaviours (American Diabetes Association, 2017 \& SEMDSA Guideline Committee, 2017). SEMDSA recommends that diabetes education provided to people living with diabetes, should be considerate of individual needs, literacy, socio-economic, cultural and educational differences (SEMDSA Guideline Committee, 2017). Effective diabetes self-management should be centred around encouraging shared decision making between the person living with diabetes and health care professionals. The components of diabetes self-care involve healthy eating, physical activity, and tobacco smoking cessation, and weight management, medication adherence, self-monitoring of blood glucose and caring for feet. Due to the need for these continuous management actions, patients often describe diabetes as a demanding disease and many studies report non-compliance issues. 
A systematic review by Stephani et al. (2018) indicates poor diabetes management in subSaharan Africa (Stephani, Opoku, \& Beran, 2018). This is a serious threat to glycaemic and blood pressure control, which often leads to the development of diabetic complications, increased hospital admissions and premature mortality. Adherence to self-care behaviour is even more important in African countries, given the low resource settings in which patients are required to manage diabetes, and the lack of facilities and trained health care workers for diabetes management (Levitt, 2008). Thus, adherence to self-care behaviours is influenced by a variety of factors including resource settings, family, friends and health care providers (Shrivastava et al., 2013; WHO, 2016a). For example, health care providers are essential in keeping patients informed regarding effective care of the disease. Those with greater knowledge and understanding have greater chances of influencing the progression of the disease and the development of any diabetic complications. The role of family and friends in either inhibiting of enabling diabetes self-care, has also been recognised within the literature (Baig, Benitez, Quinn, \& Burnet, 2015; Bennich et al., 2017). For example, healthy eating amongst people with diabetes may be positively or negatively influenced by the family's diet choices. Various social determinants may act as enablers or inhibitors of self-care and influence variation in compliance to self-care activities by people with diabetes (Shrivastava et al., 2013). These include amongst others socio-economic status, health cost and education. These factors contribute to an unequal distribution in adherence to self-care practices and the distribution of diabetes across socio-economic status.

\subsection{Explaining health inequalities and its drivers}

Inequalities in health are intertwined with income inequalities (Elgar, 2010). Growing evidence points to the fact that countries with wider income distributions suffer more from socio-economic status related health problems (Elgar, 2010; Wagstaff, 2002) and that those with lower socio-economic status are affected by a multiplicity of deprivations (Ataguba, Akazili, \& McIntyre, 2011). Underlying inequalities in predisposing factors, commonly referred to as social determinants of health, are recognised as the driving force behind health inequalities. The WHO's Commission on the Social Determinants of Health points out that inequalities in health are often a result of the circumstances individuals find themselves in, where they are born, where they live, their ethnicity or socio-economic status (Solar \& Irwin, 
2010). The conceptual framework suggested by the WHO categorises these into structural (for example income, education, gender, race) and intermediary factors (material circumstance, health care system, biological factors and behavioural factors) that influence and explain ill-health (Solar \& Irwin, 2010) (see Figure 1-3). The literature suggests that observed inequalities in health are partly a reflection of inequalities in these social determinants of health (Wagstaff, 2002). This indicates that in order to reduce health inequalities, policies should target a reduction in the inequalities in these social determinants of health (Wagstaff, 2002).

In the case of diabetes, whilst there are no known modifiable risk factors for type 1 diabetes, type 2 diabetes risk factors include both modifiable and non-modifiable factors, such as age, ethnicity, unhealthy diet, urbanisation, physical inactivity, obesity and family history of diabetes (IDF, 2001). Although there is evidence that non-modifiable (genetic) risk factors such as age, ethnicity and family history of diabetes increase the risk of developing diabetes, the recent increases in diabetes prevalence are more closely linked to the modifiable (acquired) lifestyle risk factors such as unhealthy diets, obesity and physical inactivity (IDF, 2001). 
Figure 1-3 Commission on the Social Determinants of Health conceptual framework

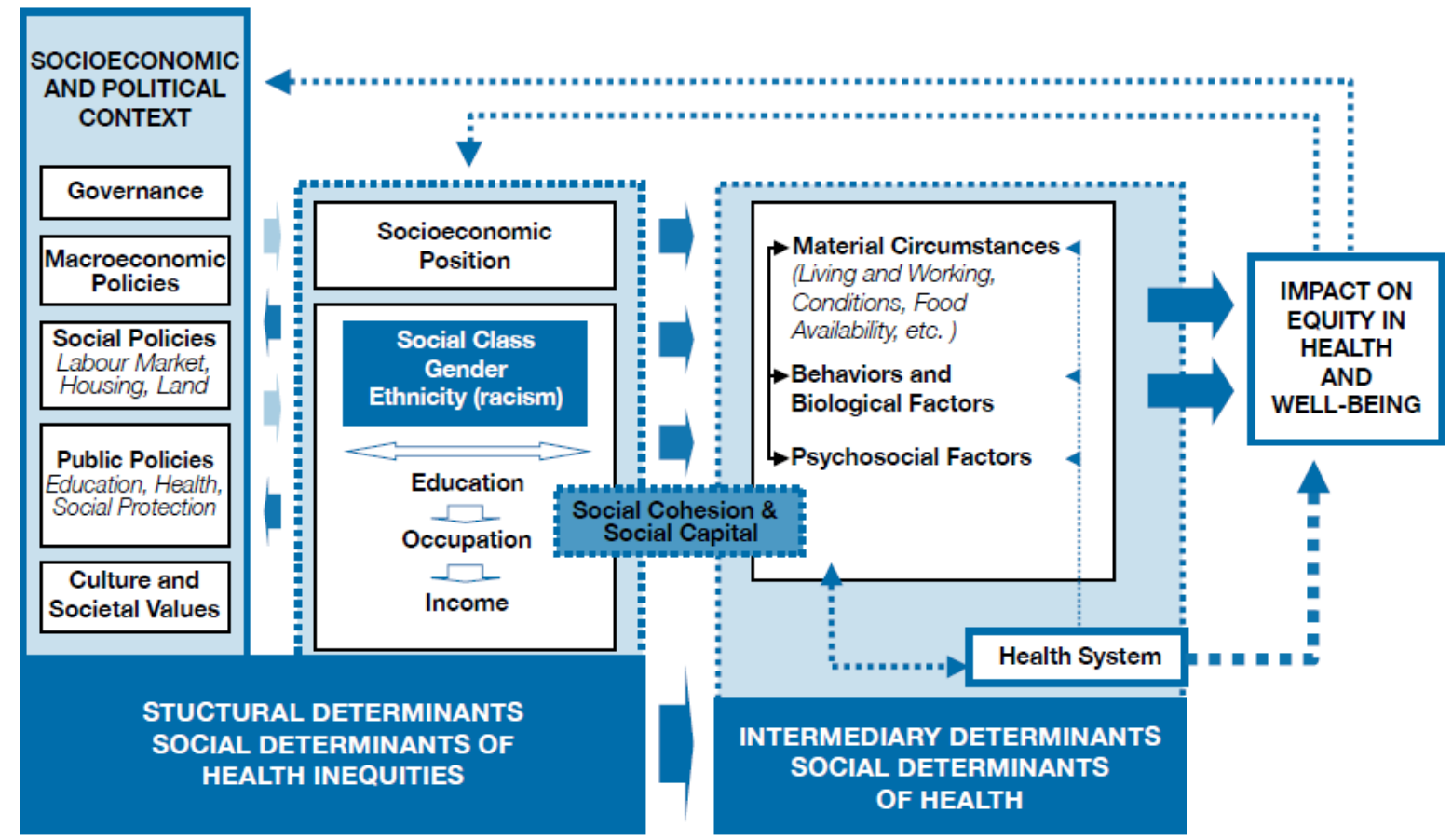

Source: Solar \& Irwin, 2010.

\subsubsection{Health Inequalities in South Africa}

The pervasive nature of health inequalities and their disproportionate burden on lower socioeconomic groups also holds true for South Africa. Persisting social disparities, poverty and inequality in socio-economic status continue to influence the disproportionate distribution of social determinants of health and ultimately exacerbate inequalities in health and well-being (Ataguba et al., 2011). Although much progress has been made in reversing apartheid's discriminatory policies, infectious diseases and NCDs continue to plague the health and wellbeing of most South Africans (Mayosi \& Benatar, 2014). Previous research in South Africa demonstrates the presence of inequalities in health, which favour the rich, especially so for the major categories of ill-health and disability (Ataguba et al., 2011). Even for NCDs, which were previously seen as diseases of affluence, research shows an increased burden of disease amongst the poor (Ataguba et al., 2011). In the case of South Africa and given its huge income inequalities, there are large disparities in the distribution of social determinant of health. For example, the country has high levels of poverty, unemployment and health care access. Whilst the right to health care is enshrined in the South African constitution, this is not the reality that many individuals face within the country. Variation in access to health care in 
South Africa is evident in the structure of the health care system, which is discussed in sections to follow. This has a huge impact on the poor and further translates into the marked health inequalities.

\subsection{Socioeconomic outlook and burden of disease in South Africa}

In order to contextualise the economic impact of diabetes and diabetes management in South Africa, it is important to obtain an understanding of the country's socio-economic outlook. Furthermore, a short description and contextualisation of the burden of disease in South Africa is necessary in order to understand the environment in which diabetes exists and is managed.

\subsubsection{General socioeconomic outlook in South Africa}

South Africa is described as a middle-income country with a per capita GDP of US\$6797 in 2018 (Bogoev, Dessus, Hanusch, Kwaramba, \& Whyte, 2018). According to Statistics South Africa, the country has a population estimate of 57.7, million in mid-2018, residing in 9 provinces, with the Black African population constituting approximately $81 \%$ of the population, with approximately $30 \%$ of the population being younger than 15 years (Statistics South Africa, 2018b). The country had an unemployment rate of $29 \%$ in quarter 2 of 2019 (Statistics South Africa, 2019b), and an annual consumer price inflation of 4.5\% in June 2019 (Statistics South Africa, 2019a). Over the last decade, the global economic crisis has also affected South Africa, leading to a deceleration in the economic growth rate. Real GDP growth went down to $0.8 \%$ in 2018 from 1.3\% in 2017 (Republic of South Africa National Treasury, 2019). Contractions in the agriculture, mining and construction sectors were the main reasons for the slowdown in the economic growth (Republic of South Africa National Treasury, 2019). Poverty is also marked with approximately $19.3 \%$ of the population living close to the poverty line (Bogoev et al., 2018). South Africa is a country with huge inequalities as reflected in the Gini coefficient, a standard metric of income inequality, of 0.63 (Hurlbut, 2018). Inequality within the country is perpetuated by the legacy of exclusion, lack of inclusive consumption growth and low intergenerational mobility. The levels of poverty and inequality within the 
country have nutritional and health outcome influences on the management of diabetes and other NCDs.

\subsubsection{The burden of disease in South Africa}

A country's burden of disease refers to the mortality, morbidity, injuries and disabilities that arise as a result of disease. South Africa has a life expectancy of 64 years in 2018, an infant mortality rate of 36.4 per 1000 live births in 2018 (Statistics South Africa, 2018b). According to the causes of death statistics released by South Africa, the top ten underlying leading causes of death in South Africa in 2016 were tuberculosis (6.5\%), diabetes mellitus (5.5\%), other forms of heart disease (5.1\%), cerebrovascular diseases (5.1\%), Human Immune Deficiency Virus (HIV) (4.8\%), hypertensive diseases (4.4\%), influenza and pneumonia (4.3\%), other viral diseases (3.6\%), ischaemic diseases (2.8\%) and chronic lower respiratory diseases (2.8\%) (Statistics South Africa, 2018c). Overall, 57.4\% of all deaths in 2016 were due to NCDs, $31.3 \%$ were due to communicable diseases and $11.2 \%$ were due to external causes such as accidents and suicide (Statistics South Africa, 2018c). The epidemiological change that South Africa is going through, is evident in the shift from communicable diseases being the biggest cause of death to NCDs (Statistics South Africa, 2018c). Whilst communicable diseases are transmissible directly or indirectly from one person to another, and are largely preventable and immediately treatable, NCDs are non-transmissible, last for longer periods of time and are known to progress with time.

Although communicable diseases are highly common in developing countries, the adoption of "Western" lifestyles and rapid urbanisation has resulted in the increased prevalence of NCDs. South Africa is described as undergoing a quadruple burden of disease, referring to the burden of both NCDs (such as diabetes), communicable diseases (such as HIV/AIDS), an epidemic of maternal, new-born and child health as well as violence and injury. Communicable diseases are more common in developing countries where poverty is high, patient education and health literacy are low. The main communicable disease health problems in South Africa are HIV and tuberculosis pandemics. In 2018, 7.52 million people were estimated to be living with HIV in South Africa. Also, approximately $19 \%$ of those within the age group 15-49 years were HIV positive (Statistics South Africa, 2018b). South Africa has 
made crucial strides in the fight against the spread of HIV, having the largest antiretroviral programme in the world with over 3 million people on treatment (South African National AIDS Council, 2015). This has placed unprecedented pressure on the provision of health care. The emergence of NCDs in both rural and urban areas places further strain on the health care system as the demand for chronic and long-term health care increases. Much of the disease burden is preventable as it is attributable to lifestyle factors, such as alcohol abuse, unhealthy diets, smoking and lack of physical activity. According to a national burden of disease study, NCDs accounted for $21 \%$ of years of life lost (Bradshaw et al., 2003). Without a strong health care system and disease prevention measures put in place, the country would only succeed in subverting efforts to meet the targets set out in the SDGs. Adoption of preventative measures and integration of NCD health care into the health care system is essential to preventing an NCD epidemic.

\subsubsection{National response to NCDs in South Africa}

The Strategic Plan for the Prevention and Control of NCDs in South Africa provides direction for the achievement of the targets set out by the 2011 Summit on the Prevention and Control of NCDs (National Department of Health, 2013). This plan is closely aligned with the WHO Global Health Action Plan for the prevention and control of NCDs (WHO, 2013). Both plans support the role of intersectoral collaborations in ensuring that cost-effective and evidencebased interventions are implemented. However, as stated in the Strategic Plan, detailed information on NCDs in South Africa is constrained due to inadequate surveillance and research. In order to influence the plan and related policy, further research into the patterns and implications of diabetes and chronic communicable disease comorbidity is imperative. As set out in the country's Strategic Plan for the Prevention and Control of NCDs, the three primary approaches identified to minimise the burden of NCDs such as diabetes are: (1) focusing on prevention and health promotion; (2) health care systems strengthening to enable diagnosis and control; (3) to expand monitoring, surveillance and research of NCDs and their risk factors (National Department of Health, 2013). Consistent information on the burden of diabetes, its associated risk factors becomes increasingly important in achieving the targets set out in these action plans and the achievement of Universal Health Coverage in South Africa. 


\subsection{Overview of the South African health care system}

The challenges associated with diabetes costs and diabetes management in South Africa can be better understood in relation to the country's general health care system.

\subsubsection{Health care provision in South Africa}

The South African health care system is often described as being two-tiered, consisting of a large public sector and a much smaller private sector (National Department of Health, 2011a, 2015a). The private and public health care sectors exist in parallel separated along socioeconomics lines resulting in inequitable access to health care (Health Policy Project, 2016). The roots of the health care system in South Africa can be found in the apartheid legacy of racial and economic inequalities (Ataguba \& Alaba, 2012). As embodied in the NHI policy document, the post-apartheid government inherited a health care system that was devised to create racial inequalities. The democratic government then initiated a process to create a non-discriminatory health-care system. Unfortunately, the current two-tier health care system is divided along socio-economic lines and has perpetuated inequalities in health outcomes (National Department of Health, 2015a). Although significant progress has been made in the delivery of health care, several drawbacks still exist.

The private sector is often described as offering world class services, staffed by approximately $70 \%$ of the county's medical doctors, serving approximately $16.2 \%$ of the population and financed mainly through private medical schemes (Mayosi \& Benatar, 2014; National Department of Health, 2011a). Private health care professionals provide health care directly to patients through private hospitals or private surgeries. Health care in the public sector is designed to be available to all South Africans and provided via a hierarchical referral health care system consisting of free primary health care and a three-tiered hospital structure (district, regional and central/tertiary). Primary health care clinics or community health care centres are the first point of contact for the patient. Patients are then referred to district, regional, provincial or academic hospitals depending on their needs. The public sector is poorly resourced, overstretched, faces human resources and aging infrastructure constraints (Mayosi \& Benatar, 2014; National Department of Health, 2011a; Ranchod et al., 2017). 
Patient experiences with public hospitals in South Africa include overcrowded facilities and long waiting times (National Department of Health, 2011a).

In an international comparison of the health care systems of forty-eight developed and developing countries, South Africa's public health care system was ranked number forty-one whilst its private health care system was ranked sixth (Burger \& Christian, 2018). Inequalities between the two sectors are not only visible in these ratings or in the number of health professionals within each sector but also in the disproportionate distribution of health care facilities. Those who access the private sector health care facilities, are reported to be much closer to a health care facility when compared to the public sector users that are more geographically dispersed and thus further away from health care facilities (Ranchod et al., 2017). The high inequalities between the private and public sectors manifest in the poor health outcomes visible in the country (African National Congress. 3rd National General Council, 2010; Council for Medical Schemes, 2017).

It is the public health care sector that has to carry the responsibility of diabetes management for the majority of people with diabetes in South Africa. Insufficient consultation time, poor patient compliance and overcrowding have been cited as barriers towards the implementation of national guidelines for diabetes in this sector (Daniels et al., 2000). Even after the liberation and restructuring, the South African public health care system has poor health outcomes as it struggles to meet the demands of its population and the increased burden of disease (National Department of Health, 2011a, 2015a). Therefore, much is still required of the health care system in terms of promotive, preventive, curative and rehabilitative care within various health programmes including diabetes care.

\subsubsection{Health care funding in South Africa}

The Department of Health notes that South Africa spends at least $8.5 \%$ of its GDP on health (National Department of Health, 2011a). This percentage is split between the private sector (4.2\%) and the public sector (4.3\%). The percentage allocated to the private sector serves $16.2 \%$ of the population whilst the remaining $4.3 \%$ of GDP is spent on $84 \%$ of the population (National Department of Health, 2011a). This shows the disproportionately lower share of 
health expenditure directed towards the public sector that serves the majority of the population. The WHO recommends that countries spend at least $5 \%$ of their GDP on health care. Despite the expenditure levels in South Africa being higher than those recommended by the WHO, South Africa's health care indicators are far worse than similar middle-income countries (National Department of Health, 2011a).

Health care in South Africa is funded mainly via OOP payments, medical insurance and general tax revenues (Ataguba \& Akazili, 2010; National Department of Health, 2011a). Within the private sector, health care is mainly funded via medical insurance $(83.5 \%)$ and OOP payments (13.0\%) and accounts for 49.8\% of total health care expenditure in 2015 (WHO, 2016b). The private health care system is available to non-insured individuals who can afford to pay the private sector fees or to individuals who can afford medical aid either through employers funding their medical aid or their own personal capacity. In instances where the service is not a part of the benefits package, private health sector requires medical scheme members to pay co-payments to service providers. This type of financing tends to cater for people that fall within the medium or high-income groups (Ataguba \& Akazili, 2010). Thus, medical scheme membership has remained unaffordable and inaccessible to the majority of the population. In recent years, increases in private health care costs have influenced medical scheme enrolments as evidenced by the decline in medical scheme coverage from $17.1 \%$ in 2016 to 16.4\% in 2018 (Statistics South Africa, 2018a). This places more strain on the public health care system, as many become largely dependent on the public health care system.

Public sources make up approximately $48.3 \%$ of total health expenditure and serve mostly informal sector workers, the poor and unemployed (WHO, 2016b). Whilst in the South African public sector primary health care is accessed for free, user fees within hospitals are charged according to a Uniform Payment Fee Schedule (UPFS) in which patients are grouped into fullpaying patients and subsidised patients. Disparities in the provision of health care within the private versus the public health care sectors, are also reflected in the annual per capita expenditure on health spent in the private sector (USD 1400) versus the public sector (USD 140) (Mayosi \& Benatar, 2014). The inequalities evident between the private and public health care sector in South Africa are a reflection of pervasive income inequalities within the 
country. It is within this system that those within the lowest socio-economics groups have poor access to health care (Harris et al., 2011).

\subsection{Aims and objectives of the dissertation}

An essential step in working towards meeting the targets set out in the SDGs, is the promotion of research which facilitates the development of policies that target the attainment of the SDGs. Out of the 17 SDGs, one specifically targets health with a goal to ensure healthy lives and promote well-being for all (i.e. SDG3). Because the pathways to achieving these targets are not pre-determined and may vary per country, scientific research is an important line of action in enabling achievement of the SDG targets. Eradicating health inequalities and financial risk protection from health care costs are some of the ways in which the targets set out on SDG3 may be met.

As the IDF observes, the prevalence of diabetes is increasing and will have huge economic burden. As discussed in section 1.2 of this introduction in African countries, including South Africa, the burden of diabetes is predicted to increase at an even faster rate when compared to high income countries. This increase in prevalence will place pressure on an already strained health care system. Furthermore, in South Africa, diabetes was the second biggest underlying cause of death in 2016 (Statistics South Africa, 2018c). The increased mortality and morbidity of diabetes strikes mostly people within their productive years. This has the potential to reduce economic productivity, affect the livelihood of households, and impoverish families. Furthermore, given the huge income inequalities in South Africa, which are intertwined with health inequalities, this increased diabetes prevalence will affect individuals and households disproportionately. In order to formulate effective mitigating measures and design measures to reduce the burden of diabetes, it is important to have relevant knowledge about the distribution, inequalities, and economic impact and management practices of diabetes.

\subsubsection{Aim}

The overall aim of this dissertation is to evaluate the economic impact and the inequality aspects of diabetes in South Africa. The study aims to provide knowledge and insight that may 
help guide the development of effective equitable interventions targeted at improving health for populations at high risk.

\subsubsection{Objectives}

In order to address the main aim of this dissertation, we define the following objectives:

Objective 1: To capture the evidence on the cost of diabetes in Africa, review the methods used to calculate costs and identify areas for future research

Given the chronic nature of diabetes and that diabetes management is characterised by frequent hospital visits, the first objective of this dissertation is to capture the evidence on the overall direct and indirect costs of diabetes. Given the projected increases in diabetes in Africa and its economic implications, the review focuses on studies conducted in Africa. Moreover, a systematic review on the costs associated with diabetes in Africa has not been conducted. The review seeks to update and expand on previous reviews that have been conducted on the costs associated with diabetes globally. The purpose of the review is also to establish methods and approaches that have been previously applied in the study of diabetes health care costs. The overall focus is on the question "what are the direct and indirect costs associated with diabetes in Africa and what methods have been applied in the calculation of these costs". The results from this review provide a base for a primary study on the costs associated with diabetes in South Africa.

Objective 2: To determine the extent of the catastrophic health expenditure and impoverishment amongst people with diabetes in South Africa

There is currently no study that looks at the economic impact of diabetes on patients in South Africa, therefore a study of this nature was necessary. In particular, a study that assesses financial protection by estimating financial protection measures of catastrophic health expenditure and impoverishing spending on diabetes health care. These two measures are also critical for tracking universal health coverage. Given the huge income inequalities in South Africa, this study sought to investigate the incidence, socio-economic inequalities and determinants of catastrophic health expenditure and impoverishment amongst people with diabetes visiting two hospitals in the Tshwane district of Gauteng. It is estimated that less 
than a third of the population within the district has health insurance, the district has an unemployment rate of approximately $20 \%$ and a Gini coefficient of 0.64 . For the purpose of the study, data were collected at two public hospitals that operate out-patient diabetes clinics. The diabetes clinics are accessible to the district urban population. Given that health care in public hospitals is subsidised and provided according to a UPFS, these two clinics were suitable case studies as they provided an opportunity to establish whether the government has been successful in protecting patients from financial hardships associated with accessing health care facilities.

Objective 3: To estimate socio-economic inequalities in diabetes and examine the contribution of lifestyle factors to diabetes inequalities in South Africa.

As explained above, previous research has revealed the existence of inequalities in the prevalence of diabetes in South Africa. Such research has however relied on self-reported data, thus, excluding individuals with undiagnosed diabetes. The IDF reports that in 2017, approximately half the people between the ages of 20-79 years with diabetes were undiagnosed. The proportion of undiagnosed people with diabetes is estimated to be over $60 \%$ of people with diabetes in Africa. Thus, this study sought to investigate the socioeconomic inequalities in diabetes by making use of both data on undiagnosed diabetes and self-reported data on a previous diabetes diagnosis. Given the role of modifiable lifestyle risk factors in diabetes prevalence, this study also sought to establish the contribution of lifestyle factors to the prevalence of diabetes in South Africa.

Objective 4: To determine the inequalities in diabetes self-care practices and the determinants of diabetes self-care practices in South Africa

Evidence on the burden of diabetes would be incomplete without considering how individuals cope with managing the chronic illness. Once diagnosed, the management of diabetes involves adhering to various diabetes self-care practices including but not limited to, healthy diet, self-monitoring of blood glucose levels, not smoking, limited alcohol consumption, routine foot care and adherence to diabetic medication as recommended by health care providers. The international literature provides evidence that adherence to these self-care behaviours varies and is influenced by a variety of factors such as socio-economic status. 
Therefore, using quantitative data, this study sought to explore the inequalities and factors associated with diabetes self-care practices amongst people with diabetes attending two public diabetes health care clinics in the Tshwane health district. In order to address the objectives of this study, findings from the quantitative data are complemented with findings from qualitative data on diabetes health management.

Objective 5: To investigate the self-reported prevalence of and factors associated with diabetes and cardiovascular comorbidity in South Africa

Diabetes self-care is an essential element to prevent or delay the occurrence of diabetes complications (IDF, 2017). Hence, after assessing self-care management practices amongst people with diabetes, it becomes necessary to establish the prevalence of factors associated with diabetes CVD comorbidities. Individuals with diabetes are at an increased risk of experiencing CVDs. Between the years 2005 and 2015, approximately USD 84 billion of GDP was consumed by CVD and diabetes in LMICs (IDF, 2017). The presence of CVD in-patients with diabetes leads to a reduction in the quality of life and an increase in health care related costs. More than $25 \%$ of all CVD expenditure is due to diabetes (IDF, 2017).

\subsection{Conceptual framework}

Figure 1-4 presents the conceptual framework adapted for this dissertation illustrating the influence of various determinants on the distribution of diabetes, and where diabetes is present, the role of self-care in influencing diabetes outcomes. The framework is influenced by (1) the WHO Commission on the Social Determinants of Health conceptual framework, (2) social cognitive theory, (3) SEMDSA diabetes management guidelines, and (4) cost of illness analysis. The framework recognises that the prevalence of and inequalities in diabetes are influenced by various behavioural factors (Chapter 4). The role of these factors is driven by income inequalities. The framework further recognises that once diagnosed, adherence to diabetes self-care practices is influenced by various factors, which too are driven by income inequalities (Chapter 5 and 6). These factors and others have a role to play in the development of diabetes complications (Chapter 7). Furthermore, the presence of diabetes is associated with various costs. The dissertation categorises these costs into indirect, direct medical, direct 
non-medical and intangible costs (Chapter 2), which may influence the development of and inequalities catastrophic heath expenditure and impoverishment (Chapter 3 ).

The key concepts covered within the dissertation are inequalities and economic burden. For the measurement of economic burden, the dissertation makes use of two methods that estimate catastrophic health expenditure and impoverishment. There are many methods that have been used within the literature for the estimation of catastrophic health expenditure and impoverishment. For robustness, our dissertation employs two methods, the WHO method and the 'Ataguba' method. These methods are discussed in detail in Chapter 3. For the estimation of health inequalities, the literature provides six methods, range, Gini coefficient and Lorenz curve, a pseudo-Gini coefficient and pseudo-Lorenz curve, the index of dissimilarity (ID), the slope index of inequality (SII), the relative index of inequality (RII) and the concentration index. It is established that the $\mathrm{Cl}$ satisfies the minimum criteria for a health inequality measure, as such we make use of the $\mathrm{Cl}$ throughout the dissertation. The $\mathrm{Cl}$ is discussed in detail in Chapter 3 of the dissertation. 
Figure 1-4: Conceptual framework

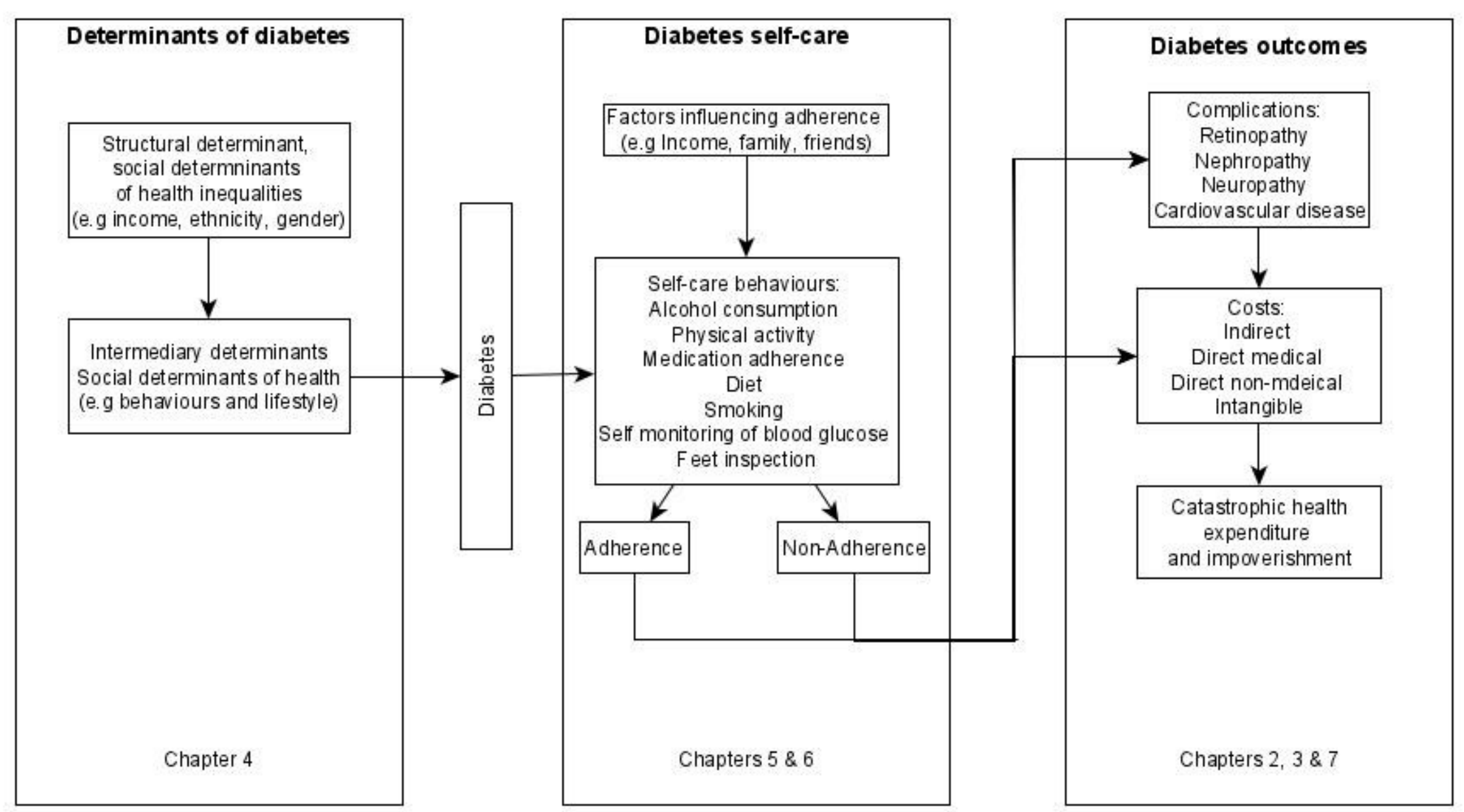




\section{$1.8 \quad$ Datasets}

The empirical analyses of this dissertation are based on two datasets: (1) the 2012 South African National Health and Nutrition Examination Survey (SANHANES-1); (2) primary data collected from three diabetes health care clinics in Tshwane health district in the Gauteng Province.

SANHANES-1 was conducted in 2012 to measure the health and nutrition status of the South African population. Ethical clearance was received from the Human Sciences Research Council (HSRC) (REC 6/16/11/11) and informed consent was provided by each participant. The survey was conducted by the HSRC. A multi-stage stratified cluster sampling approach was applied in the survey. Using the 2001 survey, a total of 1000 enumeration areas (EAs) were selected. The selection of EAs was stratified by province and locality type. A total of 500 EAs were then selected from the master sample of 1000 EAs based on the socio-demographic profile of South Africa. A random sample of 20 visiting points was then selected from the EAs yielding a sample of 10000 households. The final sample consisted of 6306 households that were valid and occupied. The dataset included 26806 individuals. The survey covered both a clinical and a questionnaire component. Three questionnaires were administered, a household questionnaire, an individual questionnaire and a child questionnaire. For the clinical component blood samples and physical examinations were conducted at various facilities such as school halls, city halls, community centres and primary health care facilities. The three questionnaires were administered at the participant's home. A detailed description of the data collection can be found in Chapter 3 of the dissertation.

The second dataset used in this dissertation, is collected at diabetes health care clinics in Tshwane. Tshwane is one of the five districts in the Gauteng province. Quantitative data were collected at two diabetes health care clinics located at two public hospitals. Both hospitals serve similar catchment populations and are accessible to the districts' urban population and outlying areas. The cross-sectional survey collected information of diabetes related issues, health expenditure and diabetes management practices. Face-to-face interviews were conducted with patients visiting the two hospitals during the data collection periods in 2017 and using a structured questionnaire. A total of 405 patients agreed to be interviewed. Ethical 
clearance for the study was obtained from the Research ethics committee of the HSRC (ref 14/23/11/16) and the University of Pretoria Research ethics committee (Protocol number 114/2017). Each participant provided written consent. A detailed description of the data collection can be found in Chapter 3 of the dissertation. To complement the findings from the quantitative data collected on diabetes management, qualitative data were collected from these same public sector diabetes clinics and one private diabetes health care clinic in Tshwane. A description of the methodologies applied in collecting this data can be found in Chapter 6 of the dissertation.

\subsection{Outline of the dissertation}

The dissertation is divided into eight chapters including this introductory chapter. Unlike the 'traditional type' dissertation that provides one chapter on the dissertation methods, the structure of this dissertation is such that each chapter comes with its own methods section and can be read as a stand-alone study. The chapters address the objectives set out in this introductory chapter. Below we provide an outline of each chapter.

Chapter 2 of the dissertation captures the evidence on the cost and economic burden of diabetes in Africa and the methods used to calculate these. The review is focused on articles that reported on original research findings on the cost of illness (COI) in diabetes published over the period 2006 - 2016. The chapter provides evidence on the huge direct costs associated with diabetes and also highlights the limited evidence on the indirect costs associated with diabetes in Africa. This corresponds with objective 1.

As outlined above, there is a lack of evidence on the costs associated with diabetes in South Africa. In particular, there are no studies that assess the economic impact of diabetes on people with diabetes in South Africa. Thus, Chapter $\mathbf{3}$ is focused on capturing the evidence on the OOP payments made by people with diabetes in South Africa. The chapter focuses on the public health sector, which is used by the majority of the South African population. This corresponds with objective 2. Using data collected from patients visiting two out-patient diabetic clinics, situated at two public hospitals in Tshwane health district, the study provides evidence on the incidence, socio-economic inequalities and determinants of catastrophic health expenditure due to diabetes. The differences in health care costs, catastrophic health 
expenditure and impoverishing effects of diabetes health care across socio-economic groups is analysed and discussed in the chapter.

Given the huge income inequalities observed in South Africa and their ties with health inequalities, Chapter 4 presents an analysis of the socio-economic inequalities in undiagnosed diabetes, diagnosed diabetes and total diabetes (diagnosed + undiagnosed). The chapter draws upon data from the 2012 SANHANES-1 and similar to Chapter 3 applies the Erreygers Concentration indices to access socio-economic inequalities. The chapter also investigates the contribution of lifestyle factors to diabetes inequalities in South Africa. This corresponds with objective 3 .

As explained above, lifestyle factors are not only important for the prevention of diabetes but also for its management. Chapter $\mathbf{5}$ is focused on assessing the self-care behaviours of people with diabetes. Just as chapter 3 , the analysis draws on data collected from the two out-patient diabetic clinics situated at two public hospitals in Tshwane. Consistent with chapter 4, the study places emphasis on inequality estimates by estimating the inequalities in diabetes selfcare practices of dietary diversity, medication adherence, physical activity, self-monitoring of blood glucose, avoiding smoking and limited alcohol consumption. The chapter also investigates the factors associated with adhering to these diabetes self-care practices. This corresponds with objective 4 .

The analysis presented in Chapter 5 is extended in Chapter 6 where we use qualitative data to explore the challenges people with diabetes face in diabetes self-care and the factors that promote and enable them to adopt recommended diabetes self-care practices. Like Chapter 5 , this chapter focuses on objective 4 and is devoted to the analysis of qualitative data collected from patients visiting one private diabetes clinic and data from the same diabetic clinics from which the quantitative data used in Chapter 3 and 5 were collected. The presented analysis allows us to draw conclusions regarding the importance of multi-sectoral approaches to diabetes self-care.

Non-adherence to diabetes self-care practices is linked to the emergence of diabetic complications. In Chapter 7, we fill in the research gap on diabetes and CVD comorbidity in South Africa. The study presents evidence on the self-reported prevalence of and factors 
associated with diabetes and CVD comorbidity in South Africa. Similar to Chapter 4, this study makes use of data from the 2012 SANHANES-1. The conclusions on addressing diabetes CVD comorbidity are drawn from this chapter. This corresponds with objective 5 .

The dissertation is concluded in Chapter $\mathbf{8}$ which presents the discussion of the main findings outlined in the preceding chapters. These findings are discussed in relation to the dissertation objectives. Policy implications in relation to the prevalence, costs, treatment and management of diabetes are also discussed. 
Chapter 2

Cost of diabetes mellitus in Africa: a systematic review of existing literature

This chapter draws upon:

Mutyambizi C, Pavlova M, Chola L, Hongoro C, Groot W. Cost of diabetes mellitus in Africa: a systematic review of existing literature. Globalization and Health. 2018;14(1):3 


\section{Abstract}

\section{Background:}

There is an increasing recognition that non communicable diseases impose large economic costs on households, societies and nations. The aim of this paper is to capture the evidence on the cost of diabetes in Africa, review the methods used to calculate costs and identify areas for future research.

\section{Methods:}

A desk search was conducted in Pubmed, Medline, Embase, and Science direct as well as through other databases, namely Google Scholar. The following eligibility criteria were used: peer reviewed English articles published between 2006 and 2016, articles that reported original research findings on the $\mathrm{COI}$ in diabetes, and studies that covered at least one African country.

\section{Results:}

Twenty-six articles are included in this review. Annual national direct costs of diabetes differed between countries and ranged from I\$3.5 billion to I\$ 4.5 billion per annum. Indirect costs per patient were generally higher than the direct costs per patient of diabetes. Outpatient costs varied by study design, data source, perspective and health care cost categories included in the total costs calculation. The most commonly included health care items were drug costs. In studies that reported both drug costs and total costs, drug costs took a significant portion of the total costs per patient. The highest burden due to the costs associated with diabetes was reported in individuals within the low-income group.

\section{Conclusion:}

Estimation of the costs associated with diabetes is crucial to make progress towards meeting the targets laid out in Sustainable Development Goal 3 set for 2030. The studies included in this review show that the presence of diabetes leads to elevated costs of treatment which further increase in the presence of complications. Various methods are used in the estimation of diabetes health care costs and the costs estimated between countries differ significantly.. 


\subsection{Background}

Diabetes mellitus is now one of the major challenges for many of the health care systems in Africa (A. P. Kengne, June-Rose McHiza, Amoah, \& Mbanya, 2013). For a long time, diabetes was considered a disease of affluence (M. B. Jakovljevic \& Milovanovic, 2015) and Africa was considered relatively free from the disease (André Pascal Kengne, Amoah, \& Mbanya, 2005). Epidemiological transition, demographic and nutrition changes have often been cited as the major driving forces in the rapid increase of the number of individuals with diabetes in Africa (M. Jakovljevic \& Getzen, 2016; A. P. Kengne et al., 2013). In 2015 diabetes was one of the leading causes of NCDs death, contributing 1.5 million deaths globally (Wang et al., 2016) and 321100 deaths in the African region (IDF, 2015). A staggering 79\% of these deaths in Africa occurred among people below the age of 60 (IDF, 2015). The IDF estimates that the number of people with diabetes in Africa will increase from 14.2 million in 2015 to 34.2 million in 2040 (IDF, 2015). More than half of the adults with diabetes in Africa live in some of the region's most populous countries: South Africa, the Democratic Republic of Congo, Nigeria and Ethiopia (IDF, 2015).

The increase in prevalence and premature mortality due to diabetes imposes huge financial costs to households and governments (WHO, 2014a) whist placing immense pressure on the already overstretched health care systems in Africa (J. C. Mbanya, 2007). The urgent need to address the NCD pandemic is now also entrenched in one of the 17 SDGs (United Nations General Assembly, 2015). Amongst other targets, SDG 3 affirms a commitment to ensure a reduction by one third in premature mortality due to NCDs and the achievement of universal health coverage by 2030 . The accomplishment of this target will amount to a reduction in NCD prevalence, which will potentially offset the costs associated with NCDs and will contribute to the elimination of inequalities in health care costs.

Individuals with diabetes are likely to experience one or more chronic illnesses such as heart disease, and kidney disease (IDF, 2015). As the prevalence of diabetes increases, the macro vascular and micro vascular complications associated with the disease will make it a very costly disease to manage, consuming an ever-increasing vast amount of resources and national health care budgets (WHO, 2016a). Notwithstanding the paucity of data on diabetes 
in Africa, the IDF estimates that Africa spends $7 \%$ of its health care budget on diabetes (IDF, 2015). Health care expenditure due to diabetes in 2015 was USD 3.4 billion and is estimated to increase to USD 5.5 billion in 2040 (IDF, 2015). However, these estimates are uncertain because $66.7 \%$ of people with diabetes in Africa are assumed to be undiagnosed (IDF, 2015). This unmet need for diabetes diagnosis is a result of weak health care systems in many African countries that fail to screen patients for diabetes (Manne-Goehler et al., 2016). Efforts to manage the disease in African countries are further hampered by a lack of diabetes education and the role of traditional healers (André Pascal Kengne et al., 2005). On a continent in which resources are limited and health finance is heavily reliant on OOP payments, African governments grapple with the costs of diabetes management (A. P. Kengne et al., 2013; J. C. Mbanya, 2007).

The usefulness of the results presented in COI studies has often been questioned due to the wide variation in methods used (Bloom, Bruno, Maman, \& Jayadevappa, 2001; Tarricone, 2006). Despite this COI studies have been conducted across a wide range of diseases and continue to play an important role in conducting full economic evaluations of treatments and other health care interventions (Bloom et al., 2001; Molinier et al., 2008). An assessment of diabetes costs in African countries is important for various reasons. The prevalence of diabetes is rapidly growing and mostly affects young adults. This has the potential to affect economic productivity and it also threatens the livelihood of many families within the region. The region has a high unmet need for diabetes diagnoses and treatment (Manne-Goehler et al., 2016). It is heavily reliant on OOP health care financing and is reported to have the lowest spending on diabetes when compared to other regions (IDF, 2015; J. C. Mbanya, 2007). An estimation of the costs associated with diabetes can give insight into the benefits of disease prevention and can facilitate the design and adoption of cost-effective treatment options. This is particularly important for countries like South Africa that are looking to achieve universal health coverage.

Recent reviews on costs of diabetes have almost exclusively focused on developed countries, the United States of America and European countries, with a small number of African countries included (Ettaro, Zhang, \& Engelgau, 2004; Ng, Lee, Toh, \& Ko, 2014; Seuring, Archangelidi, \& Suhrcke, 2015). Reviews that focused on diabetes in Africa did not strictly look 
into the COI but aimed to investigate the epidemiology of diabetes and its complications as well as the challenges in access to diagnosis and care for diabetes (Gill, Mbanya, Ramaiya, \& Tesfaye, 2009; Levitt, 2008; J. C. N. Mbanya, Motala, Sobngwi, Assah, \& Enoru, 2010). Hall et al. (2011) and Kengne et al. (2013) build up on these studies on diabetes in Africa by retrieving information on the costs associated with diabetes (Hall, Thomsen, Henriksen, \& Lohse, 2011; A. P. Kengne et al., 2013). A study published in 2007 by Jean Claude Mbanya concludes that the costs associated with diabetes consume a huge proportion of annual health budgets in Sub-Saharan African countries, many of which already run health care budget deficits (J. C. Mbanya, 2007). Although there was limited information on diabetes COI in Sub-Saharan Africa at the time, the authors draw from other studies and show that diabetes consumed up to 8.1\% of the health care budget in Tanzania for the year $1989-1990$ and 3.5\% in Cameroon for the year 2001-2002 (J. C. Mbanya, 2007). Our review expands on these previous studies and reviews the costs of diabetes within Africa. The review seeks to: (1) capture the evidence presented in the literature on the overall direct and indirect costs of diabetes in Africa that have been published since 2006; (2) review the methods that have been used to calculate the costs of diabetes in Africa; (3) identify areas for future research.

\section{$2.2 \quad$ Methods}

This literature review was guided by the Preferred Reporting Items for Systematic Reviews and Meta-Analysis (PRISMA) guidelines (Moher, Liberati, Tetzlaff, Altman, \& Group, 2010). The guidelines provide a minimum set of reporting items aimed at improving the quality of systematic reviews and meta-analyses. The collection and review of articles was conducted between January and May 2017.

\subsubsection{Literature search strategy}

A desk research was conducted in order to identify articles for inclusion. The literature search was undertaken in PubMed, EMBASE, Medline and Science Direct. Searches were also conducted in the WHO Global Health Library and IDF. Additional searches were also undertaken in Google Scholar and manual searches were also undertaken in Google in order to identify publications that may not be indexed in international databases. As an example, our PubMed search strategy is provided in Appendix A. All searches were conducted in 
January 2017. Keywords were carefully selected to ensure that all relevant material was included and to also avoid including unnecessary articles. The keywords and search strategy applied was checked by an experienced university librarian. The search terms were adapted for each database using (1) terms for African (Africa OR each country in Africa) AND (2) terms related to diabetes (diabetes OR diabetes mellitus OR diabetic) AND (3) terms denoting costs (expenditure OR cost OR economic burden OR health care cost OR cost of illness).

Following the search for articles, the next step involved duplicates removal, this was done using EndNote. In the first level of screening, article titles were screened by one reviewer (CM) to identify studies to include in the review. If based on the article title alone, it was unclear whether the study was relevant with regard to our research question, the article's abstract was then screened. The third and final step involved assessing for eligibility via fulltext review to determine if the inclusion and exclusion criteria were satisfied. Articles were then downloaded for a full-text review. In circumstances where the reviewer or a library assistant failed to access the full-text, emails were sent to the corresponding authors whose contact details could be obtained, requesting a copy of the missing studies. Reference lists of all eligible articles and reference lists of excluded reviews were also screened for additional relevant material. In every step of the selection process if there was any doubt regarding the inclusion/exclusion of an article another author was consulted.

\subsubsection{Inclusion criteria}

Eligibility criteria were determined by relevant elements of the PICOS guidance for undertaking literature reviews (Tacconelli, 2010). Papers were included if the population consisted of individuals with diabetes (type 1 or type 2), interventions and comparators was not applicable, outcomes consisted of the direct and indirect costs of diabetes and the study designs were observational or intervention studies, hypothetical studies and surveys. Articles were included if they: (1) were published between 2006 and 2016; (2) were in English language; (3) were published in peer-reviewed journals; (4) reported original research findings on diabetes $\mathrm{COI}$ or health expenditure data; (5) covered at least one African country as defined by the United Nations. Articles were excluded if they: (1) were economic evaluation studies that reported on costs derived from another study or publication; (2) only reported 
costs related to diabetes prevention; (3) were studies that did not provide original research or details on how costs were calculated; (4) did not provide patient specific costs; (5) were conference abstracts or poster presentations; (6) were animal studies.

\subsubsection{Extraction of information}

In order to extract information from studies, we developed two extraction tables. One table was used to extract details such as year published, research objectives, study design and types of costs estimated. To present information on cost data across studies, we grouped the costs as follows: outpatient costs, inpatient costs, cost of drugs and combined costs. The combined costs category included studies in which inpatient and outpatient costs per patient were not separated. This category also included indirect costs due to loss of income, disability and premature mortality.

Two adjustments were made in the presentations of costs. First, in cases where relevant unit costs were not provided, these were calculated wherever possible using the information provided in the study. Second, in order to increase comparability of costs across studies we converted costs into international dollars (further denoted with the I\$ sign) using the 2015 purchasing power parity estimates. We followed the approach used by Seuring et al. (2015). Adjustments were made for each study in which costs were not presented in the country's local currency. Costs for studies in which the USD exchange rate at the time of costing was provided, were adjusted using the provided exchange rate. For studies in which the exchange rate was not provided, we used the average exchange rate for the costing period based on the FX currency converter (OANDA). Costs presented in local currencies were immediately converted into international dollars. To estimate the purchasing power parity adjusted costs, we then applied a cost converter web-based tool developed by the Campbell and Cochrane Economics Methods Group Evidence for Policy (CCEMG) and Practice Information and Coordination Centre (EEPPI Centre). Costs in their original costing year and local currency were first adjusted for inflation to give costs in 2015 and then converted to I\$. Our study makes use of the IMF purchasing power parity method to make these adjustments to costs. For studies in which the costing year or year of data collection was not clear, we used the manuscript publication year as proxy. When a study was conducted over two different years 
(for example August 2012 to June 2013) we assumed the year of costing was the final year in which the study was concluded (i.e. 2013). In multi-country analysis studies that do not provide individual country costs estimates and reported costs in USD we applied the GDP deflator to adjust for inflation.

The second table was used to list the technical criteria applied in assessing the quality of each study. A number of check lists have been developed for the evaluation of health economics studies, most of which focus on full economic evaluations such as cost benefit analysis, costeffectiveness analysis and cost utility analysis (Higgins \& Green, 2008). Although various checklists have been developed for the appraisal of COI studies (Kleine-Budde et al., 2014; Larg \& Moss, 2011; Molinier et al., 2008; Stuhldreher et al., 2012) none of these checklists has been formally validated. The checklist used in this study is the one based on the ten-point checklist for economic evaluation developed by Drummond et al. (2005) and later adapted to COI studies by Molinier et al. (2008) and other COI studies (Becker, 2014; Costa et al., 2012; Nadège et al., 2011). We checked the quality of our systematic review based on the PRISMA 2009 checklist (see Appendix B).

\subsection{Results}

The initial search strategy yielded a total of 799 articles, from which 178 duplicates were excluded. Of the remaining 621 articles, 387 were removed during the first level of title screening and 177 removed during the second level of abstract screening leaving 57 articles for full-text evaluation. Of the 57 studies that underwent full-text review, 32 were excluded for one of the following reasons: studies were not in English, were conference presentations, full-text of articles was not available, were not peer reviewed, was a literature review of diabetes (not costs) in Nigeria, did not provide patient specific costs or presented costs that had been calculated in another included study (see Figure 2-1). Twenty-five studies were identified that met the inclusion criteria and one additional article that met the inclusion criteria, was identified after the reference screening giving the total of twenty-six articles included in the review. A list of the articles included in the review is provided in Appendix C in alphabetical order of the author's family name. 
Figure 2-1 Flow chart of study selection process

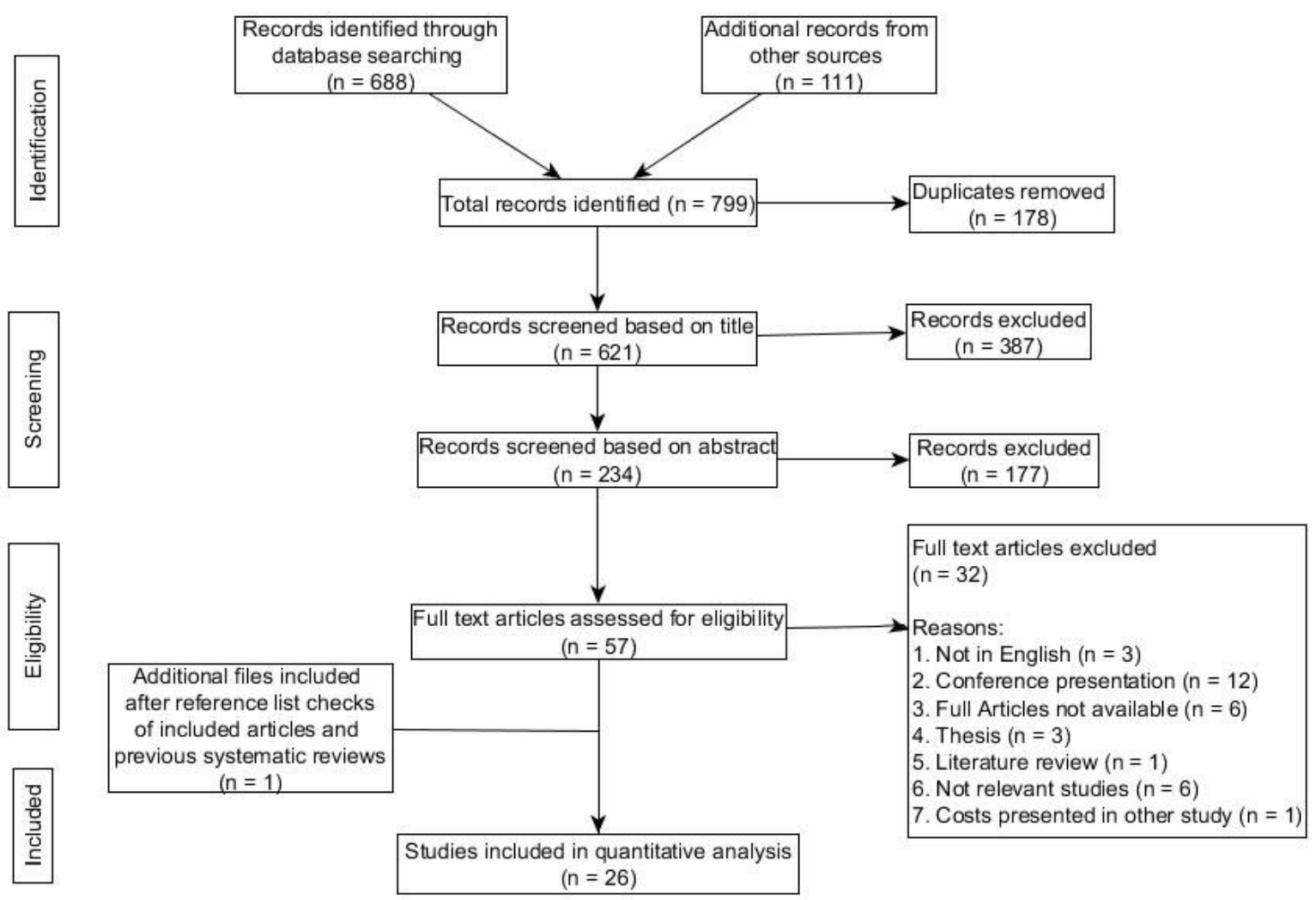

\subsubsection{General characteristics of the included studies}

The general characteristics of these twenty-six studies are provided in Table 2-1. A majority of the studies were published after the year 2010 (69\%) and the rest between 2006 and 2010 (31\%). Of the included studies, the year of costing was 2000 - 2005 for 7 studies (27\%), between 2006 and 2010 for 6 studies (23\%) between 2010 and 2016 for 9 studies (35\%) and unclear for 4 studies (15\%). It was also noticeable that most studies estimated the costs associated with diabetes for single countries (85\%) and some included multiple countries (15\%). Diabetes costs were mostly estimated for West African countries $(n=13)$ followed by East Africa $(n=6)$, Southern Africa $(n-5)$, Northern Africa $(n=2)$ and Central Africa $(n=2)$. One study estimated costs for three groups of countries within the WHO African Region.

In conducting COI studies, previous literature reviews have suggested that it is important for the study to firstly define the illness, epidemiological sources, type of costs and study perspective. After which resource consumption and unit cost data can be collected and results 
presented along with sensitivity analysis (Costa et al., 2012; Molinier et al., 2008; Nadège et al., 2011). These key methodological points are presented next.

\subsubsection{Defining the disease and population}

Overall, the majority of the studies included in this review focused on the cost of type 2 diabetes $(n=11)$, nine studies considered the costs of both type 1 and type 2 diabetes, only one study considered the costs of type 1 diabetes, whilst five studies did not clearly define the type of diabetes assessed (Table 2-1). Eighty percent of the studies focused on the general costs of diabetes, twelve percent focused on the diabetic complication of diabetic foot ulcer and eight percent focused on the cost of medication for people with diabetes. In addition to discussing the general costs of diabetes, three studies also estimated the costs of diabetic complications including but not limited to hypertension, stoke and nephropathy (Alouki et al., 2015; Ncube-Zulu \& Danckwerts, 2014; I. A. Suleiman \& Festus, 2015). The majority of the sample sizes ranged from 101-1000 (12 studies) followed by a sample size range of 1 - 100 (6 studies), > 1 million ( 3 studies) and 1001 - 2000 ( 1 study). Two studies did not provide the sample sizes (Ogle, Kim, Middlehurst, Silink, \& Jenkins, 2015; Settumba et al., 2015) and two

studies were hypothetical and calculated costs without making use of any samples (Alouki et al., 2015; Cavanagh et al., 2012). In selecting the sample to be included in the analysis, eight studies specified the age (Basu, Shankar, \& Yudkin, 2016; Elrayah-Eliadarous et al., 2010; Fadare, Olamoyegun, \& Gbadegesin, 2015; Mwavua, Ndungu, Mutai, \& Joshi, 2016; Ogle et al., 2015; Okoronkwo, Ekpemiro, Okwor, Okpala, \& Adeyemo, 2015) and three studies indicated the duration of illness (Elrayah-Eliadarous et al., 2010; Fadare et al., 2015; Mwavua et al., 2016). 
Table 2-1 General characteristics of studies included in the review

\begin{tabular}{|c|c|c|}
\hline Study characteristic & Number & Reference index in Appendix C \\
\hline \multicolumn{3}{|l|}{ Year of publication } \\
\hline $2006-2010$ & 8 & $5,8,9,11,13,18,22,25$ \\
\hline After 2010 & 18 & $1,2,3,6,7,4,10,12,14,15,16,17,18,20,21,23,24,26$ \\
\hline \multicolumn{3}{|l|}{ Year of costing } \\
\hline $2000-2005$ & 7 & $8,9,11,13,18,21,24$ \\
\hline $2006-2010$ & 6 & $1,6,12,14,17,22$ \\
\hline After 2010 & 9 & $3,7,10,15,16,19,23,25,26$ \\
\hline Not clear & 4 & $2,4,5,20$ \\
\hline \multicolumn{3}{|c|}{ No of African countries included in study } \\
\hline One & 22 & $1,4,5,6,7,8,9,10,11,12,14,15,16,17,18,19,21,22,23,25,25,26$ \\
\hline More than one & 4 & $2,3,13,19$ \\
\hline \multicolumn{3}{|l|}{ Region } \\
\hline Central Africa & 2 & 14.19 \\
\hline Eastern Africa & 6 & $5,6,11,16,19,23$ \\
\hline Northern Africa & 2 & 4.8 \\
\hline Southern Africa & 5 & $3,15,17,21,26$ \\
\hline Western Africa & 13 & $1,2,3,7,9,10,12,18,19,20,22,24,25$ \\
\hline WHO African region & 1 & 13 \\
\hline \multicolumn{3}{|l|}{ Cost Indicators } \\
\hline Direct & 26 & $1,2,3,4,5,6,7,8,9,10,11,12,13,14,15,16,17,18,19,20,21,22,23,24,25,26$ \\
\hline Indirect costs & 2 & 4.13 \\
\hline \multicolumn{3}{|l|}{ Perspective } \\
\hline Not specified & 10 & $4,5,7,8,9,11,16,17,21,24$ \\
\hline Family/patient & 7 & $2,10,12,14,18,19,20$ \\
\hline Societal & 4 & $3,13,15,25$ \\
\hline Health care system/institution & 2 & 22.23 \\
\hline Health care system and patient & 2 & 1.6 \\
\hline Government & 1 & 26 \\
\hline \multicolumn{3}{|l|}{ DM type } \\
\hline Type 1 & 1 & 19 \\
\hline Type 2 & 11 & $1,2,3,7,8,12,14,15,16,20,26$ \\
\hline Type 1 and Type 2 & 9 & $4,5,10,11,13,18,22,24,25$ \\
\hline Not specified & 5 & $6,9,17,21,23$ \\
\hline \multicolumn{3}{|l|}{ Sample sizes } \\
\hline n.a & 2 & 2.6 \\
\hline not specified & 2 & 19.23 \\
\hline $1-100$ & 6 & $7,12,15,18,21,24$ \\
\hline $101-1000$ & 12 & $1,8,9,10,11,14,16,17,20,22,25,26$ \\
\hline $1001-2000$ & 1 & 5 \\
\hline$>1000000$ & 3 & $3,4,13$ \\
\hline \multicolumn{3}{|l|}{ Epidemiological approaches } \\
\hline Prevalence & 25 & $1,2,4,5,6,7,8,9,10,11,12,13,14,15,16,17,18,19,20,21,22,23,24,25,26$ \\
\hline Incidence & 1 & 3 \\
\hline \multicolumn{3}{|l|}{ Study focus } \\
\hline General costs & 21 & $1,2,3,4,5,8,10,11,12,13,15,16,17,19,20,21,22,23,24,25,26$ \\
\hline Diabetic foot ulcer & 3 & $6,7,18$ \\
\hline Drugs & 2 & 9.14 \\
\hline \multicolumn{3}{|l|}{ Cost data source } \\
\hline Hospital or medical centre & 11 & $1,2,6,7,9,11,17,19,21,22,23,24$ \\
\hline Patients & 4 & $8,16,20$ \\
\hline Not clear & 4 & $5,14,18,25$ \\
\hline $\begin{array}{l}\text { Hospital plus other government } \\
\text { institutions }\end{array}$ & 2 & 12,24 \\
\hline Patients plus hospital & 1 & 10 \\
\hline $\begin{array}{l}\text { Who publications and various } \\
\text { individual country services }\end{array}$ & 1 & 24 \\
\hline Various data sources & 3 & $3,4,13,15,26$ \\
\hline
\end{tabular}

* One study can fall into more than one category 


\subsubsection{Epidemiological approaches}

Common epidemiological approaches in COI studies are the prevalence-based approach and the incidence-based approach (Tarricone, 2006). The prevalence-based approach is used to estimate the economic burden attributable to prevalent cases over a specific period usually one year. The incidence-based approach involves analysis of the costs of diabetes within a given period (Jo, 2014). The incidence-based approach usually follows people at similar stages of disease or diagnosis. Whilst the study by Basu et al. (2016) simulated the life course of present and new cases of diabetes over the period 2016-2025, the rest of the studies included in this review estimated the actual impact of existing cases over a period of 1 month to 2 years.

\subsubsection{Study perspective}

COI studies can be done from various perspectives. Common perspectives are the patient perspective (for example OOP payments), the employer perspective (loss of productivity), health care system perspective (hospital and primary care services), government perspective (infrastructure, support program costs) and societal perspective (loss of income while caring for the sick) (Larg \& Moss, 2011). The societal perspective is commonly applied due to its comprehensive nature. In this review, the most commonly adopted perspective was the patient perspective ( 8 studies) and respectively the societal perspective $(n=4)$, followed by the health care system perspective $(n=3)$, government perspective $(n=1)$ and the combined health care system and patient perspective $(n=1)$. Nine studies did not mention what sort of perspective was adopted, however based on reviewer interpretation we were able to classify these (see Table 2-1).

\subsubsection{Data resources}

The majority of the studies used medical centres or hospital data as their cost data sources (14 studies). In two studies cost data were obtained from patient interviews (ElrayahEliadarous et al., 2010; Okoronkwo et al., 2015), whilst one study used a combination of both patient and hospital cost data sources (Fadare et al., 2015) and three studies made use of various data sources such as published field surveys, international drug price indicators, NGOs, health insurance data, health care service providers and supplier catalogues (Basu et al., 2016; 
Boutayeb, Lamlili, Boutayeb, \& Boutayeb, 2013; Kirigia, Sambo, Sambo, \& Barry, 2009). One study that calculated the financial cost to families of children with type 1 diabetes, used cost data collected from IDF Life for a Child program centres (Ogle et al., 2015).

\subsubsection{Resource quantification}

The estimation of resource consumption can be prospectively or retrospectively performed (Tarricone, 2006). In prospective COI studies, the event would not have occurred prior to the initiation of the study. Therefore, prospective studies involve following up the patient over time. On the other hand, in retrospective studies, the events would have already occurred when the study is initiated. Three studies estimated costs prospectively (Bovet, Shamlaye, Gabriel, Riesen, \& Paccaud, 2006; Feleke \& Enquselassie, 2007; Pepper, Levitt, Cleary, \& Burch, 2007), one study used a modelling approach (Basu et al., 2016), two studies were hypothetical (Alouki et al., 2015; Cavanagh et al., 2012) and the rest of the studies estimated costs retrospectively. Eleven studies used a bottom-up approach in which either hospital records were reviewed (Abdulganiyu \& Fola, 2014; Danmusa, Terhile, Nasir, Ahmad, \& Muhammad, 2016; Enwere, Salako, \& Falade, 2006; Labhardt, Balo, Ndam, Manga, \& Stoll, 2011; Mutowo et al., 2016; Ncube-Zulu \& Danckwerts, 2014; Pepper et al., 2007; Quaye, Amporful, Akweongo, \& Aikins, 2015; Settumba et al., 2015; I. Suleiman, Fadeke, \& Okubanjo, 2006; I. A. Suleiman \& Festus, 2015) or patient interviews were conducted (Elrayah-Eliadarous et al., 2010; Fadare et al., 2015; Feleke \& Enquselassie, 2007; Ipingbemi \& Erhun, 2015; Mwavua et al., 2016; Okoronkwo et al., 2015) to gather activity data. In the study by Ogle et al. (2015), resource use was based on the IDF Life for a Child program clinical experience. Expert opinion or standard practice was used to estimate resource consumption in some studies (Alouki et al., 2015; Basu et al., 2016; Bovet et al., 2006; Cavanagh et al., 2012; Volmink, Bertram, Jina, Wade, \& Hofman, 2014). Resource consumption was estimated using various national indicators, national survey and published studies by Kirigia et al. (2009) and Boutayeb et al. (2013).

\subsubsection{Cost of diabetes mellitus}

All studies included in the review provided sufficient information to calculate per capita costs and four studies extended the national costs of diabetes (Abdulganiyu \& Fola, 2014; Boutayeb 
et al., 2013; I. Suleiman et al., 2006; I. A. Suleiman \& Festus, 2015). The national direct costs

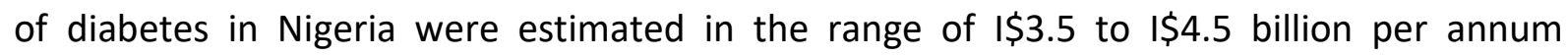
(Abdulganiyu \& Fola, 2014; I. Suleiman et al., 2006; I. A. Suleiman \& Festus, 2015) whilst in Morocco the estimated national costs (direct and indirect) are in the range I\$5.9 to I\$8.2 billion per annum (Boutayeb et al., 2013). Two studies under review quantified both direct and indirect costs (Boutayeb et al., 2013; Kirigia et al., 2009). The indirect costs were those costs associated with the loss of income, disability and premature mortality. In both studies, the human capital approach was applied in measuring costs. In both studies, the general consensus was that permanent disability accounted for the largest portion of the indirect costs. The studies that provided both direct and indirect costs show that indirect costs were higher than direct costs (Table 2-2).

The direct costs considered in the studies under review were medical and non-medical costs. Detailed costing of the studies under review is provided in Table 2-2. The table groups costs according to outpatient, inpatient and combined costs (i.e. outpatient and inpatient). Twelve studies presented outpatient costs per individual per annum (Abdulganiyu \& Fola, 2014; Alouki et al., 2015; Basu et al., 2016; Bovet et al., 2006; Elrayah-Eliadarous et al., 2010; Ipingbemi \& Erhun, 2015; Ogle et al., 2015; Okoronkwo et al., 2015; Quaye et al., 2015; I. Suleiman et al., 2006; I. A. Suleiman \& Festus, 2015; Volmink et al., 2014). It is important to note that during the estimation, cost studies used various health care components in calculating costs; therefore, results are not directly comparable. In calculating direct costs, the most commonly included health care items were drug costs, followed by diagnostic costs, medical supply or disposable costs and consultation costs, just to name a few (see Table 2-2). The presentation of out-patient costs varied across the studies. In order to facilitate comparability, we converted costs to per capita costs. Therefore, unless indicated otherwise, outpatient costs presented in Table 2-2 are average costs per individual per annum. From Table 2-2 no linear increase in costs is visible between 2002 and 2016 for individual country estimates. For example, the direct per capita out-patient costs in Nigeria varied from I\$1143 in 2004 to $\$ \$ 616$ in 2012. Costs also varied widely between countries. Wide differences are also observed for costs across the various country income groups in the study. The wide variation in costs is to a great extent a result of differences in costing methods and cost categories included in the cost estimation. When assessing costs in which costing methods 
applied and costing components included were similar, it is observed that Burkina Faso generally had higher out-patient's costs, followed by Mali, Benin and then Guinea (Alouki et al., 2015). Hospitalization costs also varied significantly within and across countries. For South Africa a notable increase in costs is visible between 2005 and 2009 (I\$ 1813 to I\$6871). Once again, the costs presented in Table 2-2 are however not directly comparable due to differences in costing methods and cost components included in the calculation of costs.

One crucial challenge highlighted in some of the studies was the OOP expenses incurred by patients. This challenge has also been reported elsewhere (J. C. Mbanya, 2007; WHO, 2014b). OOP health care costs are a hindrance to health care access and could lead to catastrophic health expenditure and impoverishment (M. Jakovljevic \& Getzen, 2016). According to the WHO African region 2014 expenditure atlas, catastrophic health expenditure is low in countries where OOP expenditure is below $20 \%$ of total health expenditure (WHO, 2014b). Where data are available, Table 2-2 also presents data from the World Bank showing OOP expenditure as a percentage of total health expenditure in each country. As shown in Table $2-2$, in 2014 South Africa was the only country in which OOP expenditure was less than $20 \%$. This is very concerning given that diabetes is a chronic illness that requires frequent health care access (M. B. Jakovljevic \& Milovanovic, 2015).

The cost of drugs was reported separately in a majority of the studies (65\%). In studies reporting both drug costs and total costs of treatment, drug costs often took up a significant portion of total costs of treatment costs, $14 \%$ - 90\% in Nigeria, $64 \%$ in Ethiopia, $53 \%$ in Sudan, $14 \%$ in the Seychelles, $4 \%$ to $7 \%$ in South Africa and $5 \%$ in Uganda (see Table 2-3). Other cost components that were commonly reported separately were diagnostic costs $(n=12)$, transportation costs $(n=9)$, and consultation costs $(n=7)$. Less than four studies reported on the costs associated with each of the following health care components separately; surgery, insurance premiums, service costs, physiotherapy, disposables, personnel, capital and diabetic diet costs. 
Table 2-2 Components of direct and indirect costs for diabetes mellitus and reflecting cost

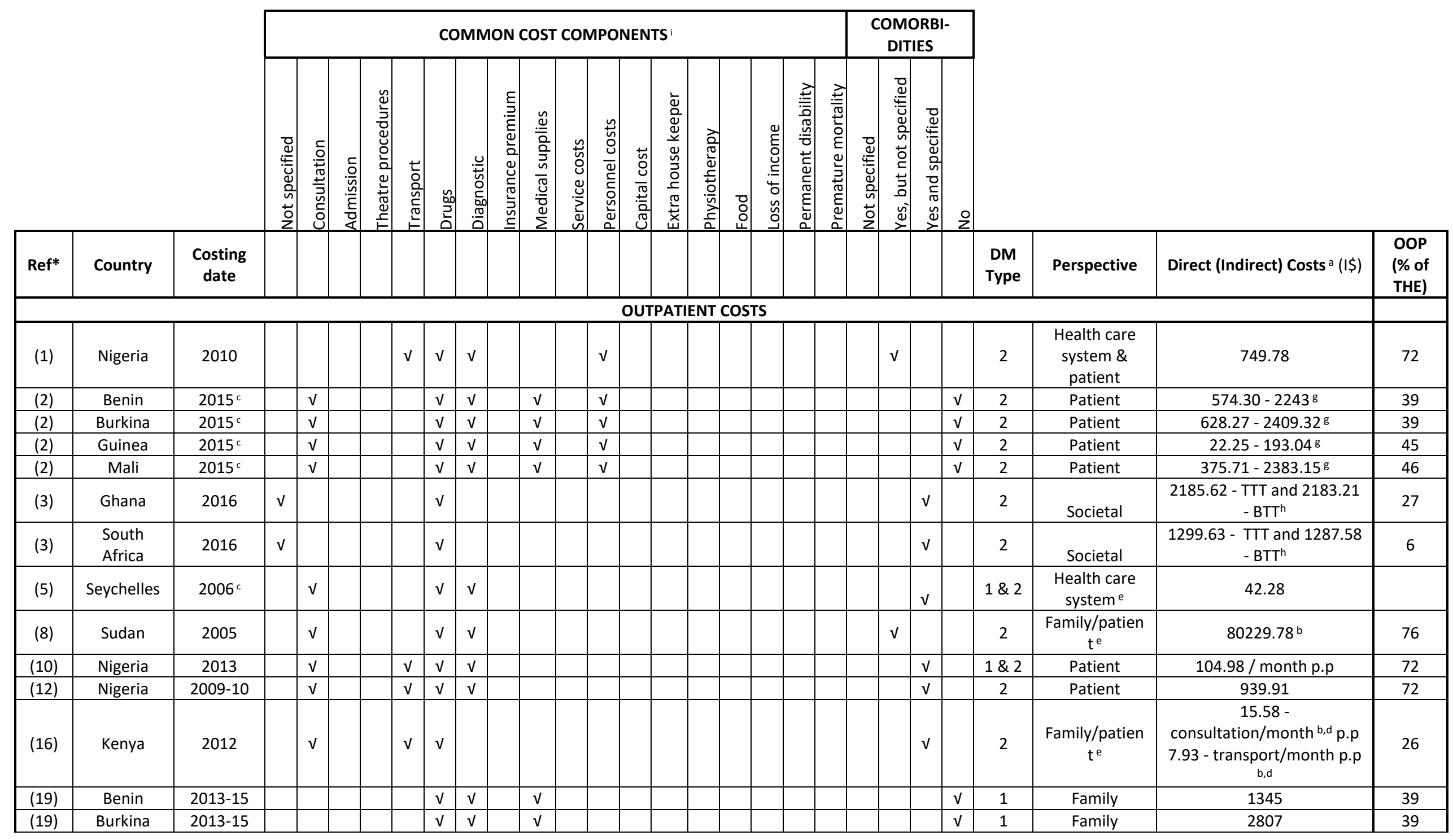




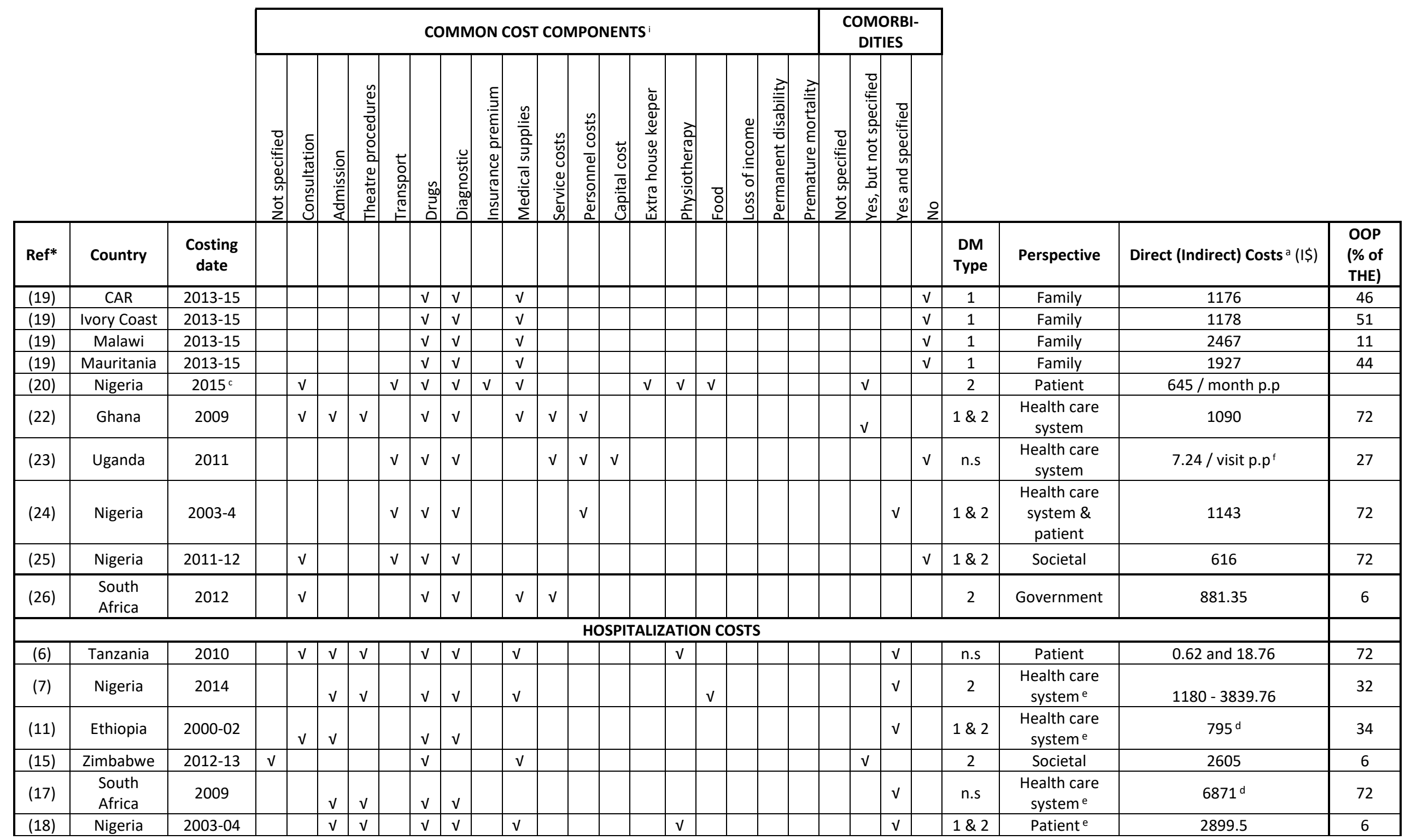




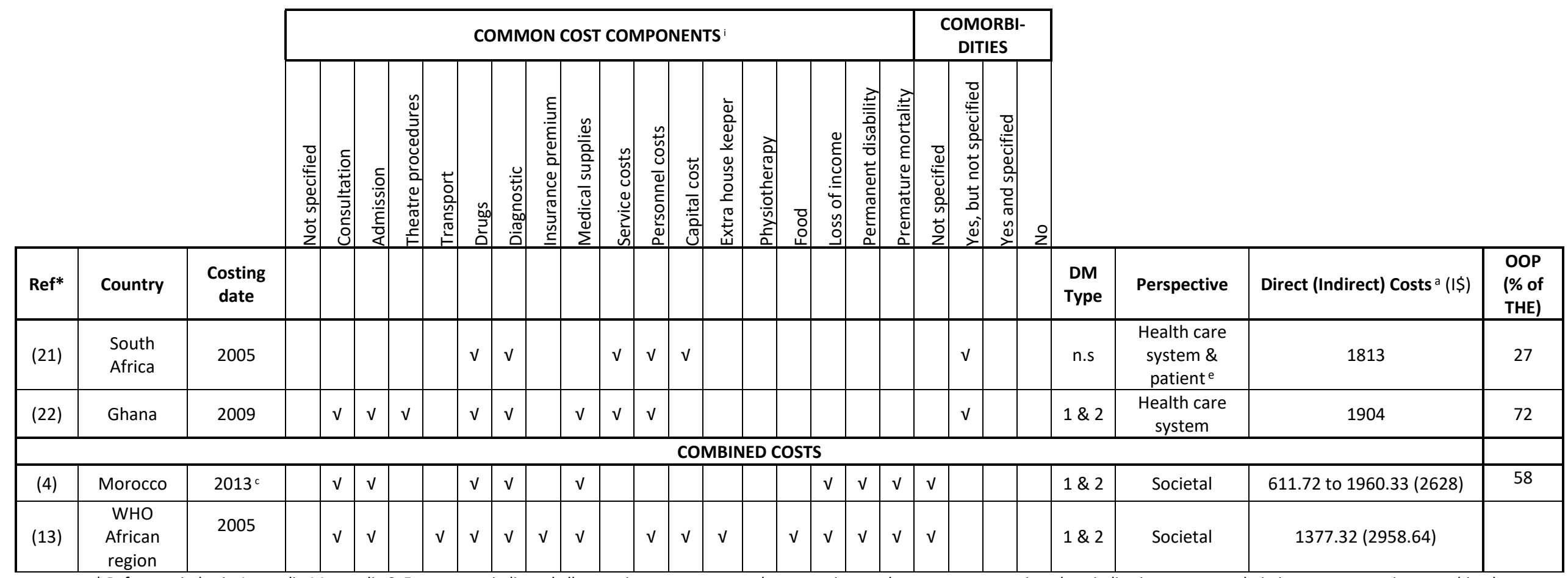

* Reference index in Appendix AAppendix C, Except were indicated all outpatient costs presented are costs incurred per annum per patient, hospitalization costs are admission costs per patient, combined costs are

costs per annum per patient, n.s: Not specified, OOP - out of pocket, THE - total health expenditure, p.d: per day.

a Presented costs are average costs except were indicated

b Presented costs are median costs

c Year of costing was unclear in study therefore the year of publication was taken as proxy

¿ OANDA FX currency converter was used to convert currency to USD

e Perspective is based on reviewer interpretation

$f \mathrm{~T}$ The study provided costs incurred at different types of health facilities - costs presented are those incurred at a hospital

$\mathrm{g}$ The study provided the minimum and maximum costs of care in the private and public health care sector of reach country. The results presented here are the maximum and minimum costs reported

${ }^{\mathrm{h}}$ Cost-effectiveness study which provided the costs of two treatment strategies (TTT and BTT) in five LMIC. Results presented in this review are for the 2 African countries studied.

i Note that categories are those visible to the reviewer or explicitly stated in the manuscript - these might not be exhaustive 
Table 2-3 Drug costs in people with diabetes

\begin{tabular}{|c|c|c|c|c|}
\hline Ref & Country & $\begin{array}{l}\text { DM } \\
\text { Type }\end{array}$ & Drug costs (I\$) & $\%$ \\
\hline \multicolumn{5}{|c|}{ Outpatient } \\
\hline 1 & Nigeria & 2 & 468.28 /annum p.p & $62 \%$ \\
\hline 5 & Seychelles & $1 \& 2$ & 6.09 / annum p.p & $14 \%$ \\
\hline 8 & Sudan & 2 & 42177.93 /annum p.p & $53 \%$ \\
\hline 10 & Nigeria & $1 \& 2$ & 80.97 / month p.p & $77 \%$ \\
\hline 12 & Nigeria & 2 & 362.41 / patient / annum & $39 \%$ \\
\hline 20 & Nigeria & 2 & $88 /$ month p.p & $14 \%$ \\
\hline 23 & Uganda & n.s & 0.36 / visit & $5 \%$ \\
\hline 24 & Nigeria & $1 \& 2$ & 1025 / annum p.p & $90 \%$ \\
\hline 25 & Nigeria & $1 \& 2$ & 420.63 / annum p.p & $68 \%$ \\
\hline \multicolumn{5}{|c|}{ Hospitalisation } \\
\hline 7 & Nigeria & 2 & 553.03 / admission & $47 \%$ \\
\hline 11 & Ethiopia & $1 \& 2$ & 511.83 / person / admission & $64 \%$ \\
\hline 17 & South Africa & n.s & 461 / patient / admission & $7 \%$ \\
\hline 18 & Nigeria & $1 \& 2$ & 1438 / admission & $50 \%$ \\
\hline 21 & South Africa & n.s & 80 / person / admission & $4 \%$ \\
\hline \multicolumn{5}{|c|}{ Others } \\
\hline 9 & Nigeria & n.s & 4.81 / patient /day & n.a \\
\hline 14 & Cameroon & 2 & 3.85 / person / month & n.a \\
\hline 16 & Kenya & 2 & $\begin{array}{l}\text { Insulin - } 10 \text { / month / p.p (average cost) Oral } \\
\text { agents - } 20 \text { / month / p.p (median cost) }\end{array}$ & n.a \\
\hline
\end{tabular}

Note: n.s - not specified, p.p - per patient, n.a - not applicable

Three studies that separately provided the costs associated with type 1 diabetes mellitus (T1DM) and type 2 diabetes mellitus (T2DM) show that the direct costs of T1DM were higher than T2DM (Quaye et al., 2015; I. Suleiman et al., 2006; I. A. Suleiman \& Festus, 2015). The hospital-based studies showed that the excess cost ratio between T1DM and T2DM ranged from 1.80 to 5.66 . The excess cost for people with diabetes versus people not living with diabetes are reported in two studies (Feleke \& Enquselassie, 2007; Ncube-Zulu \& Danckwerts, 2014). Both studies calculated hospitalization costs and found the hospitalization costs for people with diabetes to be higher than people without diabetes; a cost ratio of 1.27 to 1.50 .

Five studies included in the review estimated the costs of specific types of complications (Alouki et al., 2015; Cavanagh et al., 2012; Danmusa et al., 2016; Ogbera, Fasanmade, Ohwovoriole, \& Adediran, 2006; I. A. Suleiman \& Festus, 2015). Three of these studies focused primarily on estimating the costs associated with the diabetes complication of foot ulcer (Cavanagh et al., 2012; Danmusa et al., 2016; Ogbera et al., 2006). The costs associated with the treatment of diabetic foot ulcer in the studies varied significantly, depending on the stage 
of severity of the illness (see Appendix D). The excess costs of diabetes complications versus no complications were reported in two studies (Alouki et al., 2015; I. A. Suleiman \& Festus, 2015). Both studies showed that complications increased the burden of diabetes significantly. The incremental costs were reported for various types of complications and various countries. Among the complications investigated, the highest average cost ratios were recorded for nephropathy, diabetic foot and acute stroke. The lowest cost ratios were recorded for retinopathy, keto acidosis and hypertension. These costs details are provided in an additional file (see Appendix D).

A few other studies investigated the cost of diabetes in relation to income levels, age and by comparing costs of public versus private health care providers. Studies found that the highest burden of diabetes was amongst individuals of low socio-economic status (Ipingbemi \& Erhun, 2015; Okoronkwo et al., 2015). Okoronkwo et al. (2015) used an asset based socio-economic index to group the sample into four quartiles. The authors found that the economic burden of T2DM was highest in individuals that fell within the lowest socio-economic quartile (Okoronkwo et al., 2015). Ipingbemi and Erhun (2015) found that the economic burden of diabetes was highest in respondents who earned less that USD 125 per month (Ipingbemi \& Erhun, 2015). Whilst investigating the costs associated with diabetes in relation to age, three studies found different results. A Nigerian study by Ipingbemi and Erhun (2015) found the average outpatient cost of diabetes was highest amongst those within the age group 60 to 69 years (Ipingbemi \& Erhun, 2015). A study in Sudan finds that the outpatient cost is highest in those above 60 years of age (Elrayah-Eliadarous et al., 2010). Mutowo et al. (2016) find that hospitalization costs were lower in those older than 65 (Mutowo et al., 2016). Alouki et al. (2015) and Elrayah-Eliadarous et al. (2010) both found that medical care in the public sector was less costly when compared to the private sector (Alouki et al., 2015; Elrayah-Eliadarous et al., 2010).

\subsubsection{Quality of the included studies}

Most studies presented and explained their results in a clear way, consistent with the methodology of the study. Presentation of results was generally in agreement with the study aim and the conclusions were made in line with the results presented. More than $70 \%$ of the 
included studies carefully described the epidemiological sources, activity data, and unit costs. A few studies (24\%) included in this review did not discuss any limitations regarding the methodologies employed in calculating costs. A common weakness discussed was that studies that collected data from patient interviews were subject to recall and social desirability bias. Also, the use of one study site and small sample sizes meant that results were not applicable to other sites or national estimates. The studies by Boutayeb et al. (2013) and Kirigia et al. (2009) were also limited by factors such as the assumptions concerning the number of individuals using insulin or oral drugs, the number of people using outpatient or inpatient services, the number of diagnostic tests conducted. Hypothetical studies included in the review were subject to unavoidable differences in the treatment options provided by working groups, expert advice or physicians (Alouki et al., 2015; Basu et al., 2016; Cavanagh et al., 2012).

Costs were discounted in three studies and the discount rate chosen was $3 \%$. However, the discount rate chosen was not explained (Basu et al., 2016; Kirigia et al., 2009; Settumba et al., 2015). In the rest of the studies, the time horizon was short ( $<2$ years) and therefore, costs were not discounted. Sensitivity analysis was conducted in three studies. A one-way simple sensitivity analysis was conducted in one study (Quaye et al., 2015), another study conducted six sensitivity analysis (Basu et al., 2016) and the last one used probabilistic tests (Volmink et al., 2014).

Based on the methodological points discussed above, Table 2-4 shows the proportion of studies that met the criteria for the reporting of $\mathrm{COI}$ used in this review. For most of the studies $(60 \%)$ the answer was yes in 6 to 10 questions asked. It should be noted that the checklist has been adapted to the needs of this review and questions were benchmarked against the objectives of the study under review. A few studies did not clearly articulate or provide explicit information regarding the methodologies followed (Cavanagh et al., 2012; Ogbera et al., 2006). In some studies, the primary objective of the study was not to estimate costs hence it did not clearly explain methods adopted for cost estimation (Bovet et al., 2006; Labhardt et al., 2011; Volmink et al., 2014). 


\section{Table 2-4 Quality index score for studies included in review}

\begin{tabular}{|c|c|c|c|c|c|c|c|c|c|c|c|c|c|c|c|c|c|c|c|c|c|c|c|c|c|c|}
\hline \multirow[b]{2}{*}{ Question } & \multicolumn{26}{|c|}{ Reference index in Appendix C } \\
\hline & 1 & 2 & 3 & 4 & 5 & 6 & 7 & 8 & 9 & 10 & 11 & 12 & 13 & 14 & 15 & 16 & 17 & 18 & 19 & 20 & 21 & 22 & 23 & 24 & 25 & 26 \\
\hline 1. Was a clear definition of the illness given? & + & $\mathrm{V}$ & $\mathrm{v}$ & + & $\sqrt{ }$ & + & $\mathrm{v}$ & + & + & $\mathrm{v}$ & $\mathrm{v}$ & $\mathrm{v}$ & + & $\mathrm{v}$ & $\mathrm{v}$ & $\mathrm{v}$ & + & $\mathrm{v}$ & $\mathrm{v}$ & + & + & + & $x$ & 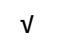 & $\mathrm{v}$ & + \\
\hline $\begin{array}{l}\text { 2. Were epidemiological sources carefully } \\
\text { described? }\end{array}$ & + & n.a & $v$ & $\sqrt{ }$ & + & n.a & $\checkmark$ & $v$ & $v$ & $v$ & $v$ & $\mathrm{v}$ & $v$ & v & $v$ & $v$ & $\mathrm{v}$ & $v$ & + & $v$ & $v$ & $\checkmark$ & $x$ & $\checkmark$ & $v$ & $x$ \\
\hline 3. Were costs sufficiently disaggregated? & $\mathrm{v}$ & $\mathrm{v}$ & $\mathrm{v}$ & + & + & + & + & + & $\mathrm{x}$ & $\mathrm{v}$ & + & $\mathrm{v}$ & $\mathrm{v}$ & $\mathrm{v}$ & $\mathrm{v}$ & + & + & $\mathrm{v}$ & $\mathrm{v}$ & + & + & $\mathrm{v}$ & $\mathrm{v}$ & + & $\mathrm{v}$ & + \\
\hline 4. Were activity data appropriately assessed? & $\mathrm{v}$ & $\mathrm{v}$ & + & $\mathrm{v}$ & $\mathrm{v}$ & + & $\mathrm{v}$ & $\mathrm{v}$ & $\mathrm{v}$ & $\mathrm{v}$ & $\mathrm{v}$ & $\mathrm{v}$ & $\mathrm{v}$ & + & $\mathrm{v}$ & $\mathrm{v}$ & $\mathrm{v}$ & + & $\mathrm{v}$ & $\mathrm{v}$ & $\mathrm{v}$ & $\mathrm{v}$ & $\mathrm{v}$ & $\mathrm{v}$ & $\mathrm{v}$ & $\mathrm{v}$ \\
\hline $\begin{array}{l}\text { 5. Were the sources of all cost values analytically } \\
\text { described }\end{array}$ & $v$ & + & $v$ & + & + & + & + & $\checkmark$ & $v$ & + & $v$ & $v$ & $v$ & + & + & $v$ & $\mathrm{v}$ & + & $\checkmark$ & $v$ & $v$ & $\checkmark$ & $v$ & $\checkmark$ & $\mathrm{v}$ & + \\
\hline 6. Were unit costs appropriately valued? & + & $\mathrm{v}$ & $\mathrm{v}$ & v & $\mathrm{v}$ & $\mathrm{v}$ & $\mathrm{v}$ & + & $\mathrm{v}$ & + & $\mathrm{v}$ & + & $\mathrm{v}$ & + & $\mathrm{v}$ & $\mathrm{v}$ & $\mathrm{v}$ & + & $\mathrm{v}$ & $\mathrm{v}$ & $v$ & + & $\mathrm{v}$ & $\mathrm{v}$ & $\mathrm{v}$ & $\mathrm{v}$ \\
\hline 7. Were the methods adopted carefully explained? & $\mathrm{v}$ & $\mathrm{v}$ & $\mathrm{v}$ & $\mathrm{V}$ & + & + & + & $\mathrm{v}$ & $\mathrm{v}$ & $\mathrm{v}$ & + & + & $\mathrm{V}$ & + & + & $\mathrm{v}$ & $\mathrm{v}$ & + & + & + & $\mathrm{v}$ & $\mathrm{v}$ & $\mathrm{v}$ & $\mathrm{v}$ & $\mathrm{v}$ & + \\
\hline 8. Were costs discounted & n.a & n.s & + & n.a & n.a & n.a & n.a & n.a & n.a & n.a & n.a & n.a & + & n.a & n.a & n.a & n.a & n.a & n.a & n.a & n.a & n.a & + & n.a & n.a & n.a \\
\hline $\begin{array}{l}\text { 9. Were the major assumptions tested in a } \\
\text { sensitivity analysis? }\end{array}$ & $x$ & $x$ & $v$ & $x$ & $x$ & $x$ & $x$ & $x$ & $x$ & $x$ & $x$ & $x$ & $\mathrm{x}$ & $x$ & $x$ & $x$ & $x$ & $x$ & $\mathrm{x}$ & $x$ & $\mathrm{x}$ & $\checkmark$ & $x$ & $x$ & $x$ & $v$ \\
\hline $\begin{array}{l}\text { 10. Was the presentation of study results } \\
\text { consistent with the methodology of the study? }\end{array}$ & $v$ & $\checkmark$ & $v$ & $v$ & $v$ & + & $v$ & $v$ & + & $v$ & $v$ & $\mathrm{v}$ & $v$ & $v$ & $\mathrm{v}$ & $v$ & $\mathrm{v}$ & $v$ & $v$ & $\mathrm{v}$ & $v$ & $\checkmark$ & $v$ & $v$ & $v$ & $\mathrm{v}$ \\
\hline \multicolumn{27}{|l|}{ Total score by study } \\
\hline VYes & 5 & 6 & 8 & 5 & 4 & 1 & 5 & 5 & 5 & 6 & 6 & 6 & 7 & 4 & 6 & 7 & 6 & 4 & 6 & 5 & 6 & 7 & 6 & 7 & 8 & 4 \\
\hline + partially & 3 & 1 & 2 & 3 & 4 & 6 & 3 & 3 & 2 & 2 & 2 & 2 & 2 & 4 & 2 & 1 & 2 & 4 & 2 & 3 & 2 & 2 & 1 & 1 & 0 & 4 \\
\hline $\mathrm{x}$ No & 1 & 1 & 0 & 1 & 1 & 1 & 1 & 1 & 2 & 1 & 1 & 1 & 1 & 1 & 1 & 1 & 1 & 1 & 1 & 1 & 1 & 0 & 3 & 1 & 1 & 1 \\
\hline n.a not applicable & 1 & 1 & 0 & 1 & 1 & 2 & 1 & 1 & 1 & 1 & 1 & 1 & 0 & 1 & 1 & 1 & 1 & 1 & 1 & 1 & 1 & 1 & 0 & 1 & 1 & 1 \\
\hline
\end{tabular}




\subsection{Discussion}

There has been growing concern over the increase in the prevalence and burden of NCDs. Endorsements such as the Moscow Declaration on NCDs in May 2011 and the Political Declaration on the prevalence and control of NCDs in September 2011 have been instrumental in raising awareness on the urgent need to place NCDs on the government agendas (Moscow Declaration, 2011; United Nations General Assembly, 2011). Following this, some countries in Africa such as Ghana and South Africa have drawn up national policies and plans for the prevention and control of NCDs (National Department of Health, 2011b; Republic of Ghana: Ministry of Health, 2012). More recently the WHO Global conference on NCDs in Uruguay decisively raised priority on the need for a multi-sectoral approach and policy coherence in order to meet the targets set out by SDG 3. All these developments are testament to the awareness surrounding the burden of NCDs such as diabetes. African countries are faced with challenges in addressing the rise in NCDs whilst still grappling with infectious diseases. This threatens to overwhelm an already overstretched health care sector and pose a challenge to economic development in Africa. There is thus growing need to assess the burden associated with these NCDs in Africa and prioritize interventions that prevent or delay the onset of diabetes.

This study contributes to the understanding of the costs of diabetes in Africa and the methods used to estimate these costs. The first objective of this review was to identify and capture the evidence presented in the literature on the overall direct and indirect costs of diabetes in Africa that have been published since 2006. The literature search identified 26 studies that met the eligibility criteria of this review. Our findings suggested that the annual economic burden of diabetes in Africa was huge. Most of these health care costs in Africa are borne by the patients and this influences the attainment of proper care due to financial constraints (J. C. Mbanya, 2007). These costs are compounded by the presence of complications which often arise as a result of the presence of risk factors (Danmusa et al., 2016). Two studies included in our review that calculate indirect costs of diabetes show that the indirect costs are higher than the direct costs. This finding is contrary to the results from a global COI study on diabetes by Seuring et al. (2015) which finds that the direct costs associated with diabetes are higher than the indirect costs (Seuring et al., 2015). It should however be noted that whilst our 
finding is based on only two studies that estimated indirect costs the finding by Seuring et al. (2015) is based on evidence from a much larger group of publications $(n=26)$. The authors find that direct costs are much higher than indirect costs mostly in high income countries (Seuring et al., 2015).

Similar to a systematic review conducted by Seuring et al. (2015), we also find that many studies did not specify the type of diabetes being reviewed. This makes it difficult to isolate and document the burden of T2DM which is the most common type of diabetes in Africa (IDF, 2015) and is largely avoidable (WHO, 2016a). Not specifying the type of diabetes also makes it difficult to make comparisons in the differences in costs due to the different types of diabetes. However, based on the three studies that separated the costs associated with T1DM and T2DM the cost of T1DM is higher than T2DM. Whilst our study finds a cost ratio of between 1.80 to $5.66 \mathrm{Ng}$ et al. (2014) find a cost ratio of 1.5 to 4.4 in their systematic review (Ng et al., 2014). The cost ratios for diabetes complications versus no complications ranged from 1.9 to 2.1 in Ng et al. (2014) whilst our review found a cost ratio of between 1.08 to 4.38. Whilst Ng et al. (2014) included a much larger group of studies; our review only includes a few articles that report on the cost of T1DM versus T2DM and diabetes complications versus no complications (Ng et al., 2014).

Like other diabetes COI reviews drug costs, diagnostic costs and consultation costs are some of the most commonly reported items ( $\mathrm{Ng}$ et al., 2014; Seuring et al., 2015; Yesudian, Grepstad, Visintin, \& Ferrario, 2014). Most studies that reported total treatment costs and cost of drugs showed that the cost of drugs weighed heavily upon the burden of diabetes, accounting for a significant portion of all direct costs (Abdulganiyu \& Fola, 2014; ElrayahEliadarous et al., 2010; Fadare et al., 2015; Feleke \& Enquselassie, 2007; Ogbera et al., 2006; I. Suleiman et al., 2006; I. A. Suleiman \& Festus, 2015). This finding is similar to Yesudian et al. (2014) who found that drug cost often constitutes $50 \%$ of the total direct costs. Many studies included in our review note that the reason for such high costs of drugs is that physicians commonly prescribe branded products. The integration of generic medicines in the writing of prescriptions is therefore crucial to reduce diabetes costs in Africa (Abdulganiyu \& Fola, 2014; Enwere et al., 2006; Fadare et al., 2015). 
Amongst the studies that reported on the cost of diabetes across various socioeconomic parameters such as income, the general consensus was that the economic burden of diabetes weighed more heavily upon those in the low-income group (Ipingbemi \& Erhun, 2015) or low socio-economic status (Okoronkwo et al., 2015). This finding is consistent with a literature review on the economic impact of diabetes in India, which also find that lower income groups generally spent more on diabetes health care (Yesudian et al., 2014).

Our second objective was to review the methods that have been used to calculate the costs of diabetes in Africa and assess the quality of the COI studies that have been included in the review. Majority of the studies were prevalence-based which is considered to be the most suitable for measuring costs related to chronic diseases (Larg \& Moss, 2011). This finding is also consistent with previous COI studies in diabetes by Yesudian et al. (2014) and Ng et al. (2014) who find that a majority of the COI studies included in their review, employed a prevalence-based study design (Ng et al., 2014; Yesudian et al., 2014). Majority of the studies were hospital-based studies involving small sample sizes; as such results might not be applicable to national estimates. A major limitation of most studies is that sensitivity analysis was not performed. The studies analyzed, applied various methodologies in the estimation of cost, hence it is very difficult to generalize results or compare the results from different studies. The differences in the methodologies applied are probably due to an absence of a formal validated methodology to be followed in COI studies (Molinier et al., 2008). According to the key methodological questions asked for most of the studies, the answer was yes in 6 to 10 questions asked (64\%) indicating an adequate quality assessment for most of the studies.

From this review it is clear that national estimates on the economic impact of diabetes in African countries are lacking. Therefore, this review provides a fragmented picture of the economic impact of diabetes in Africa. In addition, a few studies estimated the indirect costs of diabetes. Although diabetes potentially affects the ability of individuals to participate in the labour market evidence on labour markets effects is lacking. The estimates provided on the cost of diabetes did not take into account undiagnosed diabetes. As a result, it is highly likely that the aggregate costs associated with diabetes have been severely underestimated. 
Studies that provide national estimates of unmet diabetes are important for carefully estimating the national impact of the disease.

\subsection{Limitations}

The current review has some limitations. The exclusion of articles not written in English could have led to the omission of relevant articles in the area under study. The review also focused on peer reviewed articles and excluded grey literature such as academic thesis. The exclusion of this literature might introduce some bias into the review. A broader search without this restriction would yield different results. Like many other checklists, the results presented in the checklist may not be directly replicable since the results are a subjective assessment by the reviewer. In addition, the checklist used in this review does not give weighting scores on the various items included in the list. As a result, all items are given equal scoring although some items influence results more than others (Costa et al., 2012). Due to the heterogeneity in study designs and methods used in the estimation of costs, the costs presented in the study are not comparable. As a result, it was not possible to conduct a meta-analysis.

\subsection{Conclusion}

There has been a growing global focus on NCDs since the United Nations High Level Meeting on NCDSs in 2011. More recently, the specific inclusion of NCDs in the SDGs provides African countries with an opportunity to directly focus on and increase the momentum in tackling NCDs. SDG3 makes a commitment to reduce premature mortality in NCDs, to eliminate inequalities in health care costs and protect patients from diabetes induced catastrophic health expenditure, amongst others. In order to accelerate the progress in meeting SDG3 set for 2030 consideration must also be given to promoting early detection and monitoring the prevalence of NCD risk factors. The adoption of policies targeted at a reduction in harmful alcohol use, tobacco consumption and physical inactivity will contribute to a reduction in NCD prevalence and therefore health care costs.

This review provides a snapshot of the costs and economic burden of diabetes in Africa. We found that many studies on the cost of diabetes were conducted in West Africa and focused on the direct costs. This provides the West African region sufficient information to act on 
establishing cost-effective treatments that could influence a reduction in cost. Few studies estimated the economic impact of the disease using the societal perspective and few studies estimated the costs of diabetes at the national level. A common conclusion from the studies included in the review is that diabetes imposes a considerable economic burden on individuals and that amongst the cost components included in cost calculation, the cost of drugs imposed the largest burden on total costs incurred. These areas call for policies that focus on a reduction in the burden of diabetes on individuals and the promotion of prescriptions of diabetes drugs in their generic names. As the prevalence of diabetes in Africa is expected to rise, the economic burden on individuals will also continue to increase. COI studies are vital in providing information that supports economic evaluations and policy development. Future research should focus on increasing the transparency and methodological principles of COI studies. 
Chapter 3

Incidence, socio-economic inequalities and determinants of catastrophic health expenditure and impoverishment for diabetes care in South Africa: a study at two public hospitals in Tshwane

This chapter draws upon:

Mutyambizi C, Pavlova M, Hongoro C, Booysen F, Groot W. Incidence, socio-economic inequalities and determinants of catastrophic health expenditure and impoverishment for diabetes care in South Africa: a study at two public hospitals in Tshwane. International Journal for Equity in Health. 2019;18(1):73. 


\section{Abstract}

\section{Background}

Direct OOP payments for health care may cause financial hardship. This study assesses the incidence, socio-economic inequalities and determinants of catastrophic health expenditure and impoverishment amongst people with diabetes in South Africa.

\section{Methods}

Data were taken from a cross-sectional survey conducted in 2017 at two public hospitals in Tshwane, South Africa ( $\mathrm{N}=396)$. Health care costs and transport costs related to diabetes care were classified as catastrophic if they exceeded the $10 \%$ threshold of household's capacity to pay (WHO standard method) or if they exceeded a variable threshold of total household expenditure (Ataguba method). Erreygers concentration indices $(\mathrm{Cl})$ were used to assess socio-economic inequalities. A multivariable logistic regression was applied to identify the determinants of catastrophic health expenditure and impoverishment.

\section{Results}

Transport costs contributed to over $50 \%$ of total health care costs. The incidence of catastrophic health expenditure was $25 \%$ when measured at a $10 \%$ threshold of capacity to pay and $13 \%$ when measured at a variable threshold of total household expenditure. Depending on the method used, the incidence of impoverishment varied from $2 \%$ to $4 \%$ and the concentration index $(\mathrm{Cl})$ for catastrophic health expenditure varied from -0.2299 to 0.1026. When measured at a $10 \%$ threshold of capacity to pay factors associated with catastrophic health expenditure were being female (Odds Ratio [OR] 1.73; Standard Error [SE] $0.51)$, being within the $3^{\text {rd }}(0.49 ; 0.20), 4^{\text {th }}(0.31 ; 0.15)$ and $5^{\text {th }}$ wealth quintile $(0.30 ; 0.17)$. When measured using a variable threshold of total household expenditure factors associated with catastrophic health expenditure were not having children $(3.35 ; 1.82)$ and the $4^{\text {th }}$ wealth quintile $(0.32 ; 0.21)$.

\section{Conclusion}

Financial protection of people with diabetes in public hospitals is limited. This observation suggests that health financing interventions amongst people with diabetes should target the 
poor and poor women in particular. There is also a need for targeted interventions to improve access to health care facilities for people with diabetes and to reduce the financial impact of transport costs when seeking health care. This is particularly important for the achievement of universal health coverage in South Africa. 


\subsection{Background}

Since the World Health Assembly of 2005, many countries have committed to ensuring that their health care systems offer financial protection from risks of catastrophic expenditure or impoverishment due to health care payments (WHO, 2010a). More recently, through target 8 of Sustainable Development Goal 3 (SGD 3), many countries have renewed their commitment to achieving universal health coverage by 2030 as a means to prevent financial hardship and to assure equitable health care outcomes. Access to health care is considered a basic human right. However, in many African countries, OOP payments remain a hindrance to access health care and may result in financial hardships (WHO, 2014b). Globally, up to 150 million people suffer catastrophic health care expenditure yearly whilst 100 million are impoverished as a result of OOP health care payments (WHO, 2010a). It is reported that catastrophic health expenditure and impoverishment is high in countries in which OOP payments for health care are above $20 \%$ of total health expenditure (WHO, 2014b). The WHO African region expenditure atlas shows that in 2012, 37 out of 47 countries had OOP health expenditure as a percentage of total health expenditure above $20 \%$ (WHO, 2014b). In South Africa, OOP health expenditure as a percent of THE was reported to be $7 \%$ in 2014 (WHO, $2014 b)$ which is lower than over $90 \%$ of countries within the European region (WHO, 2014c).

Catastrophic health expenditure occurs when OOP health payments surpass a predefined threshold of a household resource such as household expenditure, resulting in a reduction of the household's ability to spend on other essential items and may push the household into poverty (Hsu, Flores, Evans, Mills, \& Hanson, 2018; Xu, 2005). Previous studies on the incidence of catastrophic health care expenditure in South Africa - which have not focused on diabetes health care costs - report an incidence of catastrophic health expenditure ranging from 5\% to 66\% (Babikir, Satty, \& Mwambi, 2018; Castillo-Riquelme, McIntyre, \& Barnes, 2008; S. Cleary, Birch, Chimbindi, Silal, \& McIntyre, 2013; S. M. Cleary, Birch, Moshabela, \& Schneider, 2012; Dyer, Sherwood, McIntyre, \& Ataguba, 2013; Goudge, Gilson, Russell, Gumede, \& Mills, 2009; Mudzengi et al., 2017; Negin et al., 2017). The differences in the estimated incidence is a result of differences in the study design, the data used, the different populations studied and the disease under analysis. It is reported that households with individuals with diabetes are at a high risk of experiencing financial difficulties (American 
Diabetes Association, 2018) and more likely to experience catastrophic health expenditure (Smith-Spangler, Bhattacharya, \& Goldhaber-Fiebert, 2012). Frequent visits to health care facilities for glucose monitoring, foot care and associated complications increase the cost of health care and risk being confronted with catastrophic health care expenditure.

The IDF projections indicate that within the African region, diabetes health expenditure will double to 12.3 billion international dollars by 2045 (IDF, 2017). In 2017 South Africa was reported to spend 1884 international dollars per person with diabetes (IDF, 2017). This makes South Africa the country with the second largest mean health care expenditure on diabetes in the African region and this burden is projected to grow (IDF, 2017). Despite this, as mentioned in Chapter 2 there is a paucity of research on the economic impact of diabetes for patients and households within South Africa and Africa in general, in particular studies that estimate the incidence of catastrophic health expenditure due to diabetes.

To the best of our knowledge, only a single study has investigated the incidence of catastrophic health expenditure among people with diabetes in an African country setting. Okoronkwo et al. (2015) find an incidence of catastrophic health expenditure among people with diabetes attending a tertiary health care institution in Nigeria of $45 \%$ when using a catastrophic expenditure threshold of 30\% (Okoronkwo, Ekpemiro, Okwor, Okpala, \& Adeyemo, 2015). Although the authors find that all socio-economic groups suffered catastrophic health care expenditure, the lowest socio-economic group had the highest incidence, indicating an unequal distribution of catastrophic health expenditure across socioeconomic groups (Okoronkwo et al., 2015). For people with diabetes this is very disastrous as the poor may be forced to forego other vital needs such as dietary diversity in order to attain health services. To date, in South Africa, studies that have assessed the costs associated with diabetes have done so from a health care system, societal or government perspective (Basu, Shankar, \& Yudkin, 2016; Ncube-Zulu \& Danckwerts, 2014; Pepper, Burch, Levitt, \& Cleary, 2007; Volmink, Bertram, Jina, Wade, \& Hofman, 2014). No diabetes patient costing study has been conducted and no study has assessed the incidence and socio-economic inequalities of catastrophic health expenditure for people with diabetes seeking health care in public hospitals that provide subsidised health care. 
Subsidised health care in South African public hospitals is provided according to a UPFS, which groups patients into full paying patients and subsidised patients (Mcintyre et al., 2007; National Department of Health, 2015b). Patients are classified as full paying if they are being treated within a public hospital by a private practitioner, are externally funded patients (for example funded by the road accident fund or medical scheme) or are non-South African citizens. Subsidised patients may either be fully or partially subsidised, depending on their ability to pay for health care services (National Department of Health, 2015b). Patients are classified as fully subsidised if they provide proof of being social pensioners or formally unemployed. Partially subsidised patients pay according to their income level (commonly referred to as the sliding scale means test) (National Department of Health, 2015b). Most health care charges are based on a grouping of services, meaning fees payable by patients include all costs such as consumables, overhead costs and salaries rather than itemised billing for each service (National Department of Health, 2015b). The role of such OOP payments in health care financing, even in settings with modest health care bills, is of great concern given that this is a very regressive health care financing mechanism (J. E. Ataguba \& Mclntyre, 2018). This creates the need to investigate financial protection measures in South African public hospitals.

Our study aims to (1) assess the health expenditure patterns of people with diabetes seeking health care in public hospitals that provide subsidised health care; (2) determine the incidence of poverty, catastrophic health care expenditure and subsequent impoverishment due to diabetes; ( 3 ) assess the socio-economic inequalities in catastrophic health expenditure and impoverishment due to diabetes; and (4) explore the determinants of catastrophic health expenditure and impoverishment due to diabetes. A study of this nature is important for various reasons. The prevalence of diabetes is increasing rapidly and has a significant economic impact on individuals and households. The incidence of catastrophic health expenditure and impoverishment are important indicators of the extent of financial risk protection offered by the health care system, which is particularly important within the context of universal health coverage. 


\subsection{Methods}

\subsubsection{Study setting}

This study was done in Tshwane, one of the 5 districts in the province of Gauteng, which is the most populous province in South Africa. Tshwane accounts for approximately $24 \%$ of the province's population making it the third most populous district in the province. In 2015 , the district had an unemployment rate of $21.1 \%$ and a Gini coefficient of 0.64 (City of Tshwane, 2016). The medical insurance coverage in the district was 30.5\% in 2016 (Massyn N, Tanna G, Day C, \& Ndlovu N, 2018). Approximately $87 \%$ of Tshwane's employed population work in the formal sector whilst the remainder work in the informal sector (City of Tshwane, 2016). The majority of the population in the district is African (78\%). This too is the population group with the largest proportion of people living in poverty (City of Tshwane, 2016).

Health care is provided via public and private health care facilities. The district is demarcated into 7 sub-districts with public health care being delivered at many levels via a hierarchical referral health care system. Public health care is provided via a total of 68 clinics, 8 community health centres and 9 hospitals (district, regional and central/tertiary) in 2017/18 (Massyn N et al., 2018). Each health care facility provides diabetes health care. Majority of patients accessing hospital-based health care are those in need of a higher level of health care.

\subsubsection{Ethical approval}

Ethical approval for data collection was obtained from the Research Ethics Committee of the HSRC (ref: 14/23/11/16) and the University of Pretoria Research Ethics Committee (Protocol number 114/2017). Written consent was obtained from each participant (see Appendix E for copy of consent form). Clinic managers were informed of the study and permitted access to the study sites and patients. Quality checks of all interviews and validation of completed questionnaires was conducted by the data collection supervisor.

\subsubsection{Survey}

Data collection was conducted in March to April and November to December of 2017, at two public hospitals in Tshwane, South Africa that operate diabetes clinics. The hospitals serve 
similar catchment populations and are accessible to the district's urban population and outlying areas. The objective of the survey was to collect information on diabetes-related health issues, health behaviours, health expenditure related to diabetes care and diabetes management practices. The survey consisted of face-to-face interviews conducted with people with diabetes using a structured questionnaire. All patients visiting the hospital during the data collection period, were invited to participate in the study. Patients who were severely ill and could not communicate were excluded from the survey. Four experienced research assistants were recruited to assist with the data collection process. The assistants were trained on the study protocol and data collection processes.

\subsubsection{Questionnaire}

Questionnaire development was guided by the SANHANES-1 data collection tool (Shisana O et al., 2014) and previous hospital-based studies that estimated catastrophic health expenditure (Buigut, Ettarh, \& Amendah, 2015; Okoronkwo et al., 2015). The questionnaire was then adapted to the South African public hospital context (see Appendix F). In order to ensure validity and reliability, the questionnaire was pre-tested with 8 patients at one of the hospitals and amendments made where necessary. The questionnaire required all expenditure and income data to be collected in South African Rands. The OANDA historical average exchange rate during 2017 was 13.2955 Rands per US dollar (OANDA).

\subsubsection{Sampling}

The study sample size was calculated using the single population proportion formula. The sample size was estimated at 385 , assuming a prevalence of catastrophic health expenditure of $50 \%$, confidence interval of $95 \%$ and absolute error of 0.05 . We added 115 patients to this number to account for possibility that not all invited patients would agree to be interviewed. All patients above the age of 21 visiting the hospitals diabetes clinics during the data collection period were invited to participate whilst sitting in the waiting room before consultation. No inducement or incentive was offered for participation. Thus, a total of 503 patients were invited to take part in the survey and $405(81 \%)$ patients agreed to be interviewed. Of those who refused to take part in the survey $62 \%$ were female and $72 \%$ were African. Of those who 
agreed to be interviewed, 9 were excluded from this analysis because they refused to continue with the interviews. These 9 respondents were mostly male and non-African.

\subsubsection{Statistical analysis}

Our study makes use of a COI prevalence-based approach and patient perspective to assess the OOP health expenditure incurred by people with diabetes. In this study, OOP health expenditure included payments made by people with diabetes at the hospitals. This fee does not vary for controlled versus uncontrolled diabetes and excludes OOP payments made for transportation to the health facility. However, transport costs in South Africa have previously been reported to take up a large portion of direct health care costs (Castillo-Riquelme et al., 2008; S. Cleary et al., 2013). Therefore, in estimating costs per hospital visit we make use of two approaches. The first uses the direct medical health costs only (approach 1 ) whilst the second uses both the direct medical health costs plus the direct non-medical costs of transport (approach 2). Patients were asked how much they paid for transport to the hospital and this was multiplied by two in order to estimate the costs of a return trip. In cases where patients used private vehicles, transport costs were estimated using the reported distance from patient residence to the hospital. A value for $1 \mathrm{~km}$ of R3.55 was used based on the price estimate published by the South African government (South African Revenue Services, 2017).

\subsubsection{Definition of variables}

- Household-A group of people living together who shared expenditures, was accepted to be a household.

- OOP health expenditure - Individuals were asked how much in total they paid OOP for their visit related to diabetes care. This was a fixed fee which included all services and typically included items such as consultation fees and medication

- Total household consumption expenditure $\left(\mathrm{THE}_{\mathrm{h}}\right)$ - This included expenditure made by households in order to meet their daily needs and also included expenditure on goods and services. Data were collected with specific reference to the last 30 days

- Food expenditure $\left(\mathrm{FEH}_{\mathrm{h}}\right)$ - This expenditure was measured as the amount spent on foodstuffs. Data were collected with specific reference to the last 30 days 


\subsubsection{Measuring catastrophic health expenditure and impoverishment}

The measurement of catastrophic health expenditure and impoverishment has been discussed extensively in the literature (J. E.-O. Ataguba, 2012; O'Donnell, van Doorslaer, Adam, \& Lindelow, 2008; Thomson, Evetovits, Cylus, \& Jakab, 2016; Wagstaff \& Eozenou, 2014; Wagstaff \& van Doorslaer, 2003; Xu, 2005). Regardless of the method, however, a choice has to be made regarding the threshold to use in determining catastrophic health expenditure and a choice in defining the household resources used to pay for health care (Hsu et al., 2018). Whilst the choice of threshold is arbitrary and has typically varied between $10 \%$ and $40 \%$, there have been two commonly used methods employed in defining household resources and measuring catastrophic health expenditure in the literature.

The first method by Wagstaff and van Doorslaer (2003) defines health expenditure as catastrophic when it exceeds a certain threshold of total expenditure or household income (O'Donnell et al., 2008; Wagstaff \& van Doorslaer, 2003). Critics of this method have argued that they underestimate the financial impact of health costs among poorer households due to the use of uniform thresholds (J. E.-O. Ataguba, 2012; Onoka, Onwujekwe, Hanson, \& Uzochukwu, 2011; Thomson et al., 2016). The second method by the WHO (further referred to as the 'WHO standard method') defines health expenditure as catastrophic when it exceeds a certain threshold of capacity to pay (Xu, 2005; Xu et al., 2003). There however are some reservations with this method, which are related to how exactly subsistence expenditure is measured (Thomson et al., 2016; Wagstaff \& Eozenou, 2014; Wagstaff \& van Doorslaer, 2003) and how relevant the initial estimate of the equivalence scale is (Koch, 2018). More recently, some authors have argued that in order to ensure fair and ethical measures of catastrophic health expenditure, the threshold applied in measuring it should be a function of the income distribution (J. E.-O. Ataguba, 2012; Onoka et al., 2011). Ataguba proposes a method that uses a threshold that varies with income when estimating catastrophic health expenditure (J. E.O. Ataguba, 2012). This method (further referred to as the 'Ataguba method') has recently been applied in measuring catastrophic health expenditure in Swaziland and Uganda, and is useful in countries with high inequalities (Kwesiga, Zikusooka, \& Ataguba, 2015; Ngcamphalala \& Ataguba, 2018). To check the robustness of our results, we employ both the 'WHO standard method' and the 'Ataguba method' in estimating catastrophic health 
expenditure. In both methods the incidence of catastrophic health expenditure is defined as the proportion of patients attending the diabetes clinics, whose health care expenditure due to diabetes is catastrophic. The steps followed in calculating catastrophic health expenditure and the extent of impoverishment are provided below, with each of the two methods being discussed in turn.

\subsubsection{Construction of statistical variables}

\section{- WHO standard method}

The computational steps used to generate the variables used in the method by WHO (Xu, 2005) are shown below. A detailed description of the steps followed in constructing these variables is provided elsewhere (Xu, 2005).

Step 1: Generate the food expenditure share (FES)

$F E S_{h}=F E H_{h} / T H E_{h}$

Step 2: Generate the household equivalent size (HES) as follows:

$H E S_{h}=h h_{-}$size $^{\beta}$

Where hh_size is the household size, and the coefficient $\beta$ is the value of an equivalence scale. Our study makes use of $\beta=0.56$ which was estimated from a regression equation based on 59 countries (Xu et al., 2003). A study by Koch (2018) has queried the applicability of the estimate because most of the data used to calculate it were more than 2 decades old (Koch, 2018). The use of a range of scales is therefore recommended (Koch, 2018). However, in the case for South Africa, Koch finds that although the scale has changed over the years, the choice of scale does not really affect the average incidence of catastrophic health expenditure (Koch, 2018). For the purposes of this study and consistent with recent studies (Buigut et al., 2015; Kimani, Mugo, \& Kioko, 2016), we make use of the commonly applied household scale multiplier of 0.56

Step 3: The equivalent food expenditure is obtained as follows:

$$
E F E_{h}=F E H_{h} / H E S_{h}
$$

Step 4: Identify the FES at the 45th and 55th percentile across the entire sample and name them FES45 and FES55. 
Step 5: Calculate the average of food expenditures of the households that lie within FES45 and FES55 to obtain the poverty line (PL).

Step 6: Subsistence expenditure for each household is then calculated as follows.

$S E_{h}=P L * H E S_{h}$

Step 7: Generate the household's capacity to pay $\left(\mathrm{CTP}_{h}\right)$ which is defined as the household's non-subsistence expenditure ( $\left.\mathrm{SE}_{h}\right)$ as follows:

$C T P_{h}=T H E_{h}-S E_{h}$ if $F E H_{h}>=S E_{h}$

$C T P_{h}=T H E_{h}-F_{h}$ if $\mathrm{FEH}_{h}<S E$

Step 8: Health expenditure is defined as catastrophic if OOP health expenditure exceeded a certain threshold (e.g. 10\%) of the household's capacity to pay (CTPh).

cata $_{h}=1$ if $\mathrm{OOPHE}_{h} / \mathrm{CTP}_{h}>=10 \%$

$\mathrm{cata}_{h}=0$ if $\mathrm{OOPHE}_{h} / C T P_{h}<10 \%$

There is a lack of consensus on the appropriate threshold to use when measuring catastrophic health expenditure. Lower thresholds are typically used in the total expenditure method and higher thresholds in the capacity to pay method (Hsu et al., 2018). Consistent with other studies the sensitivity of the analysis to various thresholds was tested (Aregbeshola \& Khan, 2018; Buigut et al., 2015; Ukwaja, Alobu, Abimbola, \& Hopewell, 2013). Since the selection of threshold is a normative and somewhat arbitrary choice we present results using thresholds set at $10 \%, 20 \%, 30 \%$ and $40 \%$ and leave it to the reader to determine their selection.

Step 9: A household is defined as poor if its total household consumption expenditure - THEh was smaller than its $\mathrm{SE}_{h}$ and non-poor when total household consumption expenditure - $\mathrm{THE}_{\mathrm{h}}$ was greater than or equal to $\mathrm{SE}_{\mathrm{h}}$.

$$
\begin{aligned}
& \text { poor }_{h}=1 \text { if } T H E_{h}<S E_{h} \\
& \text { poor }_{h}=0 \text { if } T H E_{h}>=S E_{h}
\end{aligned}
$$

Step 10: A non-poor household was considered impoverished by health care payments once it became poor after paying for health care

$$
\begin{aligned}
& \text { impov }_{h}=1 \text { if } T H E_{h}-\mathrm{OOPHE}_{h}<S E_{h} \\
& \text { impov }_{h}=0 \text { if } T H E_{h}-O O P H E_{h}>S E_{h}
\end{aligned}
$$




\subsubsection{Ataguba method}

In order to check the robustness of our results and due to the limitations of the WHO standard method outlined above, we also estimated catastrophic health expenditure using the method proposed by Ataguba et al. (2012). Computational steps for the method by Ataguba et al. (2012) are shown below.

Step 1: Estimate the rank dependent threshold $Z^{\prime}$ cat

$$
z_{c a t}^{\prime}=\gamma(1-\rho)^{(\gamma-1)} * Z_{c a t}
$$

where $\rho$ is the household's percentile generated when households are ordered according to income, $Z_{\text {cat }}$ is the initial threshold (an initial threshold of $10 \%$ is used in our paper), $y$ is a parameter of aversion to inequality. Following Ataguba et al. (2012), we use a value of 0.8 . However, for illustrative purposes we also present results when $y=1$. This is the case when $Z_{c a t}$ does not change across the income distribution and is similar to applying the method by Wagstaff and van Doorslaer (2003).

Step 2: Estimate the rank dependent overshoot which shows the extent to which health cost as a fraction of total household cost exceeds the threshold

$$
\begin{aligned}
& O S_{h}^{\prime}=\frac{O O P H E_{h}}{T H E_{h}}-Z_{c a t}^{\prime} \text { if } \frac{O O P H E_{h}}{T H E_{h}}>Z_{c a t}^{\prime} \\
& O S_{h}^{\prime}=0 \text { if otherwise }
\end{aligned}
$$

Step 3: Estimate the rank dependent catastrophic health expenditure head count ratio which shows the proportion of households that incur catastrophic health expenditure. Where $E=1$ when $\mathrm{OS}_{\mathrm{h}}>0$ and 0 when otherwise.

$$
H C_{h}^{\prime}=\frac{1}{N}\left(\sum_{h=1}^{N} E_{h}^{\prime}\right)=\mu_{h}^{\prime}
$$

Step 4: Using a poverty line, estimate the pre-health payment poverty head count ratio. Our study makes use of the 2017 lower bound poverty line of R758. This is a poverty line estimate generated by Statistics South Africa which takes into account both basic food and other basic needs (Statistics South Africa, 2014) and is the preferred threshold in policy making.

$$
H_{\text {pov }}^{\text {pre }}=\frac{1}{N}\left(\sum_{h=1}^{N} P_{h}^{\text {pre }}\right)=\mu_{p} \text { pre }
$$

Where $P_{h}^{\text {pre }}=1$ if adult equivalent household expenditure $T H E_{h}<$ poverty-line and $P_{h}^{\text {pre }}=0$ if otherwise.

Step 5: Estimate the post-health payment poverty head count ratio. 
$H_{\text {pov }}^{\text {post }}=\frac{1}{N}\left(\sum_{h=1}^{N} P_{h}^{\text {post }}\right)=\mu_{p}{ }_{\text {post }}$

Where $P_{h}^{\text {post }}=1$ if $T H E_{h}-O O P H E_{h}<$ poverty-line and $P_{h}^{\text {post }}=0$ if otherwise.

Step 6: Estimate the impoverishing impact of OOP health expenditure, i.e. the difference between the pre-payment and post-payment indices.

${ }^{P I}{ }_{H=}{ }_{\text {pov }}^{\text {post }}-H_{\text {pov }}^{\text {pre }}$

A detailed description of these steps is provided elsewhere (J. E.-O. Ataguba, 2012). In our study, total monthly expenditure is used as a proxy for income.

\subsubsection{Health seeking behaviours and time costs}

Data were also collected on the time spent visiting the hospital for diabetes care and workdays missed due to diabetes. Patients were asked to report in hours and minutes, how much time it took them travelling to the hospital, waiting to consult the doctor, during consultation and waiting for medication. For patients who reported being employed, the number of work days missed due to diabetes over the last 30 days, were collected using a categorical variable that took on a value of 1 when respondents took half a day, a value of 2 when respondents took 1 to 4 days, a value of 3 when respondents took 5 to 10 days and a value of 4 when respondents took more than ten days. A continuous variable was then created by taking the mid-point estimate of each category.

The indirect costs due to productivity loss were estimated for patients who reported being employed by using the monetary value of time spent seeking care and the monetary value of days missed from work. Hourly wage rate was estimated by using respondent reported monthly income and assuming patients worked 20 days a month and 8 hours a day. We then follow the method applied by Oloniniyi et al. (2019) to estimate productivity losses. The hourly wage rate was multiplied by the total hours spent seeking care and time taken off work due to diabetes over the last 30 days. 


\subsubsection{Inequalities in catastrophic health expenditure and impoverishment}

Our study makes use of the $\mathrm{Cl}$ to measure socio-economic inequalities in catastrophic health expenditure and impoverishment amongst the diabetes patients. The $\mathrm{Cl}$ is derived from the concentration curve (CC) which plots the cumulative percentage of the health variable against the cumulative percentage of the population ranked by the living standards measure (O'Donnell et al., 2008) and the $\mathrm{Cl}$ is measured as twice the area between the $\mathrm{CC}$ and the 45degree line (O'Donnell et al., 2008).

The magnitude of the $\mathrm{Cl}$ indicates a disproportionate concentration of the health measure among the poor or the rich and takes on a value between +1 and -1 . It is measured as twice the covariance of the catastrophic health expenditure/impoverishment variables and the ranking of the living standards variable $r$ all divided by the mean of the catastrophic health expenditure or impoverishment variables $(\mu)$ :

$C I=\frac{2}{\mu} \operatorname{cov}(h, r)$

Our study makes use of multiple correspondence analysis to generate the wealth index which is our living standards variable. Although there are various methods that can be used for the construction of the asset index multiple correspondence analysis is chosen because it is the preferred technique for categorical variables (Booysen, Van Der Berg, Burger, Von Maltitz, \& Du Rand, 2008). Based on items included in the questionnaire, a commonly used set of living conditions and ownership of household assets were included in constructing the wealth index. Ten household conditions and assets were considered in the analysis. The full list is as follows: housing type, water and sanitation services, ownership of a television, refrigerator, 4 plate stove, radio, cell phone, computer and car. The wealth index was later categorised into wealth quintiles. The wealth index was then applied in generating our Cls.

A negative $\mathrm{Cl}$ means that catastrophic health expenditure or impoverishment is concentrated amongst the poor whilst a positive value means it is concentrated amongst the rich. The $\mathrm{Cl}$ takes on a value of zero when there is no socio-economic inequality meaning catastrophic health expenditure or impoverishment variable is equally distributed across the sample (O'Donnell et al., 2008). Since our variables are binary, a normalisation process is required to measure inequality. This study makes use of the Erreygers corrected $\mathrm{Cl}$. 
$E(h)=\frac{4 \mu}{b-a} C I$

Where $\mu$ is the mean of the catastrophic health expenditure or impoverishment variables, $\mathrm{Cl}$ is the concentration index, $b$ is the maximum value of the variable (in this case 1 ), $a$ is the minimum value of the variable (in this case 0 ). We make use of STATA's conidex command (O'Donnell, O'Neill, Van Ourti, \& Walsh, 2016).

\subsubsection{Determinants of catastrophic health expenditure and impoverishment}

We used logistic regression to analyse the association between socio-demographic variables and catastrophic health expenditure and impoverishment. In assessing these associations, our selection of socio-demographic variables was guided by the literature (Njagi, Arsenijevic, \& Groot, 2018; Ukwaja et al., 2013; Xu, 2005). The individual and household variables included in our analysis are age, gender, race, and marital status, having children, education, employment status, household size and index quintile. Age was measured in years and was included as a continuous variable. Gender was included as a binary variable taking on the values 1 - male, 2 - female. Race was also included as a binary variable with 1 - African and 2 - non-African (white, coloured, Indian/Asian). Marital status was included as follows: 1 married/living with a partner, 2 - single. Respondents were asked if they had any children, this was included as a binary variable taking on the value of 1 when the respondent had children and 0 when the respondent did not have any children. Education was categorised as 1 - primary, 2 - secondary and 3 - tertiary education. Employment status was included as 0 - unemployed and 1 - employed. The size of the household was included as a categorical variable that took on the following values; $0-1$ to 4 household members, $1-5+$ household members. An outline and description of these variables is provided in Table 3-1. 
Table 3-1 Outline of variable used in regression analysis

\begin{tabular}{|c|c|c|}
\hline $\begin{array}{l}\text { Independent } \\
\text { variable }\end{array}$ & Description & Measurement \\
\hline Age & Age of respondent & Continuous variable \\
\hline Gender & Gender of the respondent & Dummy variable: $0=$ male, $1=$ female \\
\hline Race & Race of the respondent & Dummy variable: $0=$ African, $1=$ non-African \\
\hline Marital status & $\begin{array}{l}\text { Marital status of the } \\
\text { respondent }\end{array}$ & Dummy variable: $0=$ married, $1=$ single \\
\hline Children & Respondent has children & Dummy variable: $0=y e s, 1=$ no \\
\hline Education & Education level of respondent & $\begin{array}{l}\text { Categorical variable: } 1=\text { Primary, } 2=\text { Secondary, } \\
3=\text { Tertiary }\end{array}$ \\
\hline $\begin{array}{l}\text { Employment } \\
\text { status }\end{array}$ & $\begin{array}{l}\text { Employment status of } \\
\text { respondent }\end{array}$ & Dummy variable: $0=$ unemployed, $1=$ employed \\
\hline Household size & $\begin{array}{l}\text { Total household size of } \\
\text { respondent }\end{array}$ & $\begin{array}{l}\text { Dummy variable: } 0=1-4 \text { individuals, } 1=5+ \\
\text { individuals }\end{array}$ \\
\hline Index quintile & $\begin{array}{l}\text { Wealth index generated via } \\
\text { multiple correspondence } \\
\text { analysis and later categorised } \\
\text { into quintiles }\end{array}$ & $\begin{array}{l}\text { Categorical variable: } 1=\text { quintile } 1,2=\text { quintile } 2 \text {, } \\
3=\text { quintile } 3,4=\text { quintile } 4,5=\text { quintile } 5\end{array}$ \\
\hline
\end{tabular}

The statistical analysis was conducted using STATA version 13. In order to allow for the skewed distributions, all household income and expenditure related data are presented as means (standard deviations) and medians (percentiles). Our study reports proportions for categorical variables.

\subsection{Results}

\subsubsection{Descriptive statistics}

The characteristics of our study sample are shown in Table 3-2. The mean age for our sample was 52 years. The majority of the respondents were female (61\%). Our sample was predominantly African (76\%). About $59 \%$ of respondents reported being single, $86 \%$ reported having children, $66 \%$ reported having secondary education, and $63 \%$ were unemployed. The majority of respondents had a household size of 1-4 persons (64\%). 
Table 3-2 Study sample characteristics

\begin{tabular}{lrrr}
\hline Variable & $\mathbf{N}$ & Mean & Std. Dev. \\
\hline Age & 386 & 51.62 & 14.65 \\
Gender & & & \\
$\quad$ Male & 155 & $39 \%$ & 0.48 \\
$\quad$ Female & 240 & $61 \%$ & 0.48 \\
Race & & & \\
$\quad$ African & 300 & $76 \%$ & 0.42 \\
$\quad$ Non-African & 93 & $24 \%$ & 0.42 \\
Marital status & & & \\
$\quad$ Married & 162 & $41 \%$ & 0.49 \\
$\quad$ Single & 231 & $59 \%$ & 0.49 \\
Children & & & \\
$\quad$ Yes & 337 & $86 \%$ & 0.34 \\
$\quad$ No & 55 & $14 \%$ & 0.34 \\
Education & & & \\
$\quad$ Primary & 65 & $17 \%$ & 0.37 \\
$\quad$ Secondary & 255 & $66 \%$ & 0.47 \\
$\quad$ Tertiary & 68 & $18 \%$ & 0.38 \\
Employment status & & & \\
$\quad$ Unemployed & 236 & $63 \%$ & 0.48 \\
Employed & 137 & $37 \%$ & 0.48 \\
Household size & & & \\
1-4 & 249 & $64 \%$ & 0.48 \\
5+ & 142 & $36 \%$ & 0.48 \\
\hline Note: N - number of observations. Std. Dev. - Standard deviation
\end{tabular}

\subsubsection{Cost of illness and household expenditure}

Table 3-3 demonstrates that the mean total household expenditure for our sample was R4213 (1 US\$ = 13.30 Rands) (median value R2810). Household food expenditure averaged R1687 (median R1500). Subsistence expenditure averaged R1003 (median R1016) and capacity to pay averaged R3325 (median value R1730). 
Table 3-3 Individual and household expenditure

\begin{tabular}{lrrrrrr}
\hline Variable & N & Mean & $\begin{array}{r}\text { Std. } \\
\text { Dev. }\end{array}$ & Median & p25 & p75 \\
\hline Household costs per month & & & & & & \\
$\quad$ Total Household expenditure & 380 & 4213 & 5190 & 2810 & 1600 & 5000 \\
$\quad$ Household food expenditure & 385 & 1687 & 1258 & 1500 & 900 & 2000 \\
$\quad$ Subsistence expenditure & 391 & 1003 & 303 & 1016 & 689 & 1151 \\
$\quad$ Capacity to pay (CTP) & 380 & 3325 & 5133 & 1730 & 911 & 3725 \\
$\quad$ Direct medical cost per patient per hospital visit & & & & & & \\
$\quad$ Health care cost & 377 & 53 & 167 & 40 & 0 & 65 \\
$\quad$ Health care cost (including transport) & 361 & 132 & 209 & 96 & 50 & 150 \\
Direct non-medical cost per patient per hospital visit & & & & & & \\
$\quad$ Transport cost & 376 & 79 & 119 & 40 & 20 & 91 \\
$\quad$ Food cost during hospital visit & 289 & 30 & 153 & 20 & 0 & 30 \\
$\quad$ Time Lost per patient (hours) & & & & & & \\
$\quad$ Time taken off work (30 day period) & 100 & 11 & 7 & 16 & 4 & 16 \\
$\quad$ Time taken visiting hospital & 151 & 7 & 2 & 7 & 6 & 9 \\
$\quad$ Total time lost & 153 & 14 & 8 & 11 & 8 & 22 \\
Indirect costs per patient & & & & & & \\
$\quad$ Time taken off work (30 day period) & 100 & 429 & 615 & 250 & 110 & 450 \\
$\quad$ Time taken visiting hospital & 151 & 277 & 369 & 161 & 83 & 323 \\
$\quad$ Total time lost & 153 & 553 & 786 & 323 & 113 & 652 \\
\hline
\end{tabular}

Note: All monetary values are presented in South African Rands, Note: N - number of observations. Std. Dev. Standard deviation

Our analysis suggests that the mean OOP health expenditure per out-patient visit related to diabetes care was R53 (median value R40). The mean round trip transport cost was R79 (median R40). When we combine transport costs and health costs, we find an average of R132. The OOP health expenditure was statistically significantly different across the wealth index quintiles. Households in quintile 5 pay more for health services relative to other quintiles (see Figure 3-1). Other direct non-medical costs incurred by patients included expenses on food whilst waiting at the hospital. The mean food costs was R30 (median R20). 
Figure 3-1 Mean of OOP health expenditure for diabetes care by socio-economic status

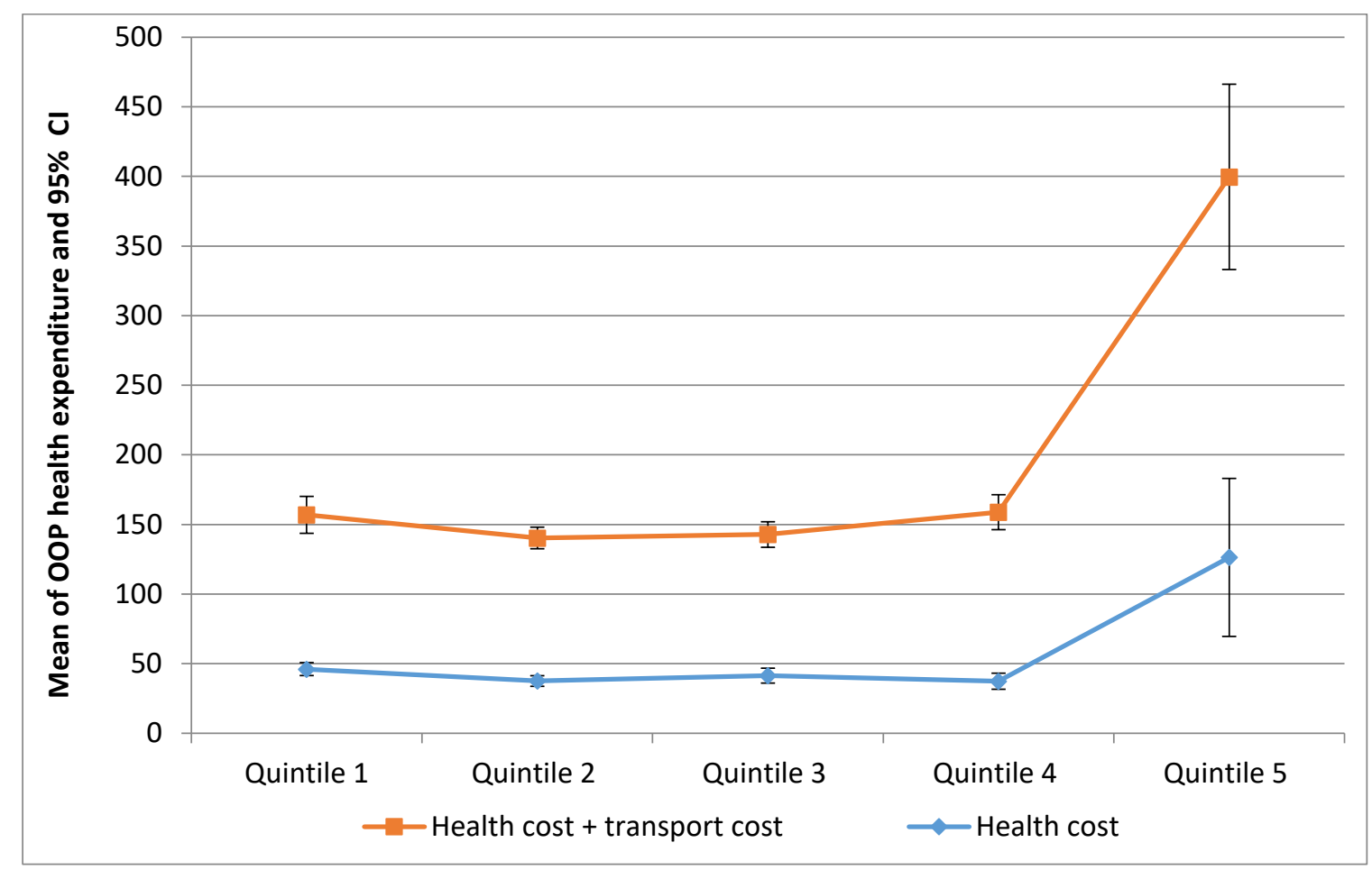

Note: All monetary values are presented in South African Rands. The error bars are $95 \%$ confidence intervals

Our estimation of productivity losses is restricted to those who reported being employed and reported their income $(n=153)$. Our findings show that out of a sample of 100 patients who reported missing work over the past 30 days due to diabetes, the average time lost was 10.6 hours. No respondent reported taking more than 10 days off work due to diabetes related illness. The average time for a hospital visit was 7 hours. The mean estimated indirect costs for missing work over the past 30 days and for a hospital visit was R 429 and R 277 respectively. When we combined the average time for a hospital visit and the time taken off work due to diabetes, the total time lost was 14.5 hours. The mean estimated indirect costs for both time spent seeking health care and time taken off work was R 553 (see Table 3-3).

\subsubsection{Catastrophic health expenditure and impoverishment patterns}

In estimating catastrophic health expenditure and impoverishment due to health care costs, we employed both the WHO standard method and the Ataguba method (see methods section). As mentioned earlier, we considered catastrophic expenditure due to direct OOP payments for diabetes care (approach 1) and also catastrophic expenditure due to both direct OOP payments for diabetes care plus direct non-medical costs of transport (approach 2). The 
sensitivity of the analysis to different thresholds was tested and results presented in Table 3-4. Due to the differences in the computational steps used in the construction of statistical variables (see methods), the sample sizes differ for each analytical method (WHO method and the Ataguba method). In each method observations with missing data in variables used for the construction of catastrophic health expenditure or impoverishment were excluded from the analysis.

We attempted to use the same sample for each method (WHO method and Ataguba method) as well as each approach (approach 1 and approach 2). Our final analytical sample dropped to 318 observations. As expected, this drop in sample size impacts the statistical significance of most of the analysis conducted in this chapter for each of these methods and approaches. Thus, we make use of different sample sizes for each analytical method.

Results from the WHO method show that approximately $6 \%$ of the study sample was poor. Catastrophic health expenditure for diabetes care was measured using four thresholds (10\%, $20 \%, 30 \%$ and $40 \%$ ) and decreased as we moved from the lower to higher thresholds. As expected, when we included transport costs in estimating the ratio of health care costs to capacity to pay, we find that catastrophic health expenditure measured at all four thresholds was much higher than when we exclude transport costs. This finding signifies the importance of transport costs. Using approach 1 (approach 2), catastrophic health expenditure for diabetes care varies from $2 \%$ to $9 \%$ (6\% to $25 \%$ ). Amongst those who were not poor and when we exclude transport costs, none of our study participants are impoverished due to diabetes care but when we include transport costs, $2 \%$ become impoverished.

For the Ataguba method, we make use of an initial threshold of $10 \%$ to estimate catastrophic health expenditure for diabetes care. We present results using a parameter of aversion to inequality of 0.8 (a varying threshold) and of 1 (a constant threshold of 10\%). As shown in Table 3-4, the increase of this parameter from 0.8 to 1 decreases the head count from $4 \%$ to $2 \%$ (approach 1) and 13\% to 10\% (approach 2). Using the South African poverty line of R758, we find that the poverty head count increased from $21 \%$ to $23 \%$ (approach 1 ) and $21 \%$ to $25 \%$ (approach 2 ). This translates into a $2 \%$ and $4 \%$ rise in poverty using approach 1 and approach 2 , respectively. 
Table 3-4 Catastrophic health expenditure and impoverishment related to diabetes care (\%)

\begin{tabular}{|c|c|c|c|c|c|c|}
\hline \multirow{2}{*}{ Method/Indicator } & \multicolumn{3}{|c|}{ Approach 1} & \multicolumn{3}{|c|}{ Approach 2} \\
\hline & $\mathbf{N}$ & $\%$ & Std.dev & $\mathbf{N}$ & $\%$ & Stnd.dev \\
\hline \multicolumn{7}{|l|}{ WHO Standard method } \\
\hline Poor & 375 & $6 \%$ & 0.2353 & 375 & $6 \%$ & 0.2353 \\
\hline Catastrophic 10 & 359 & $9 \%$ & 0.2813 & 343 & $25 \%$ & 0.4357 \\
\hline Catastrophic 20 & 359 & $4 \%$ & 0.2066 & 343 & $11 \%$ & 0.3143 \\
\hline Catastrophic 30 & 359 & $3 \%$ & 0.1726 & 343 & $8 \%$ & 0.2697 \\
\hline Catastrophic 40 & 359 & $2 \%$ & 0.1478 & 343 & $6 \%$ & 0.2454 \\
\hline Impoverished & 336 & $0 \%$ & 0.0000 & 320 & $2 \%$ & 0.1465 \\
\hline \multicolumn{7}{|l|}{ Ataguba method ( $y=0.8)$} \\
\hline Catastrophic head count ratio & 362 & $4 \%$ & 0.1996 & 346 & $13 \%$ & 0.3400 \\
\hline $\begin{array}{l}\text { Prepayment poverty head } \\
\text { count }\end{array}$ & 357 & $21 \%$ & 0.4059 & 341 & $21 \%$ & 0.4066 \\
\hline $\begin{array}{l}\text { Post-payment poverty head } \\
\text { count }\end{array}$ & 357 & $23 \%$ & 0.4212 & 341 & $25 \%$ & 0.4349 \\
\hline Impoverished & 357 & $2 \%$ & 0.1482 & 341 & $4 \%$ & 0.2053 \\
\hline \multicolumn{7}{|l|}{ Ataguba method ( $y=1)$} \\
\hline Catastrophic head count ratio & 361 & $2 \%$ & 0.1561 & 344 & $10 \%$ & 0.2989 \\
\hline $\begin{array}{l}\text { Prepayment poverty head } \\
\text { count }\end{array}$ & 357 & $21 \%$ & 0.4059 & 341 & $21 \%$ & 0.4066 \\
\hline $\begin{array}{l}\text { Post-payment poverty head } \\
\text { count }\end{array}$ & 357 & $23 \%$ & 0.4212 & 341 & $25 \%$ & 0.4349 \\
\hline Impoverished & 357 & $2 \%$ & 0.1482 & 341 & $4 \%$ & 0.2053 \\
\hline
\end{tabular}

Note: $\mathrm{N}$ - Number of observations in each method, Std.dev - standard deviation. Approach 1 is catastrophic expenditure due to direct health costs only, approach 2 is catastrophic expenditure due to both direct medical health costs plus the direct non-medical costs of transport. Ataguba method $(y=0.8)$ - threshold varies with household expenditure. Ataguba method $(y=1)$ - constant threshold of $10 \%$.

The proportion of people with diabetes who were poor, was highest in wealth quintile 1 . The proportion of catastrophic health expenditure for diabetes care was highest in the first wealth quintile when measured using both the WHO standard method and the Ataguba method. This shows that people with diabetes who incur catastrophic health expenditure are in fact the poor. From the Ataguba method we find that higher levels of catastrophic expenditure exist within quintile 1 when measured using a variable threshold $(y=0.8)$ compared to when measured using a fixed threshold $(y=1)$ For example, in approach 2 we find an incidence of catastrophic expenditure of $20.27 \%$ within quintile 1 when measured using a variable threshold and we find an incidence of catastrophic expenditure of $16.44 \%$ within quintile 1 when measured using a fixed threshold. Using the Ataguba method, the highest increases in the poverty head count ratio is recorded for people with diabetes in quintile 1 . For example, 
we see that OOP health expenditure for diabetic care leads to a $6.85 \%$ increase in the poverty head count ratio when using approach 1, i.e. without accounting for transportation costs. The figure for approach 2 is higher at $9.59 \%$ (see Table 3-5).

\subsubsection{Inequalities in catastrophic expenditure and impoverishment}

Table 3-6 shows the concentration indices for catastrophic health expenditure and impoverishment related to diabetes care. All statistically significant concentration indices are negative, indicating that catastrophic health expenditure for diabetes care is more concentrated amongst the poor people with diabetes. In the WHO standard method, the value of the catastrophic health expenditure index decreases as we move to higher thresholds, but also declines in statistical significance. The Ataguba method $(y=0.8)$, which uses a varying threshold, shows higher inequalities in catastrophic health expenditure when compared to the one which uses a constant threshold of $10 \%(y=1)$. However, the $\mathrm{Cl}$ for the Ataguba method $(y=1)$ are statistically insignificant. All the values for the concentration for impoverishment due to diabetes were negative, indicating that the proportion of impoverishment is concentrated amongst the poor. Because none of our study participants are impoverished due to diabetes care when estimated using the WHO method for approach one, we do not estimate this inequality. 
Table 3-5 Catastrophic health expenditure and impoverishment related to diabetes care, by wealth quintile

\begin{tabular}{|c|c|c|c|c|c|c|c|c|c|c|c|c|c|c|}
\hline \multirow[b]{3}{*}{ Indicator } & \multicolumn{7}{|c|}{ Approach 1} & \multicolumn{7}{|c|}{ Approach 2} \\
\hline & \multicolumn{5}{|c|}{ Wealth quintiles } & \multicolumn{9}{|c|}{ Wealth quintiles } \\
\hline & 1 & 2 & 3 & 4 & 5 & Total & $\begin{array}{c}\text { P- } \\
\text { value }\end{array}$ & 1 & 2 & 3 & 4 & 5 & Total & P-value \\
\hline \multicolumn{15}{|l|}{ WHO Standard method } \\
\hline Poor & $13.33 \%$ & $5.95 \%$ & $7.14 \%$ & $1.30 \%$ & $0.00 \%$ & $5.87 \%$ & 0.000 & $13.33 \%$ & $5.95 \%$ & $7.14 \%$ & $1.30 \%$ & $0.00 \%$ & $5.87 \%$ & 0.000 \\
\hline Catastrophic $10 \%$ & $18.67 \%$ & $9.52 \%$ & $7.50 \%$ & $0.00 \%$ & $5.77 \%$ & $8.64 \%$ & 0.000 & $39.73 \%$ & $30.12 \%$ & $22.97 \%$ & $14.06 \%$ & $14.29 \%$ & $25.36 \%$ & 0.021 \\
\hline Catastrophic $20 \%$ & $9.33 \%$ & $5.95 \%$ & $2.50 \%$ & $0.00 \%$ & $3.85 \%$ & $4.46 \%$ & 0.000 & $17.81 \%$ & $13.25 \%$ & $10.81 \%$ & $6.25 \%$ & $4.08 \%$ & $11.08 \%$ & 0.000 \\
\hline Catastrophic $30 \%$ & $4.00 \%$ & $4.76 \%$ & $2.50 \%$ & $0.00 \%$ & $3.85 \%$ & $3.06 \%$ & 0.050 & $15.07 \%$ & $8.43 \%$ & $6.76 \%$ & $1.56 \%$ & $4.08 \%$ & $7.58 \%$ & 0.000 \\
\hline Catastrophic $40 \%$ & $4.00 \%$ & $2.38 \%$ & $1.25 \%$ & $0.00 \%$ & $3.85 \%$ & $2.23 \%$ & 0.000 & $12.33 \%$ & $7.23 \%$ & $5.41 \%$ & $1.56 \%$ & $4.08 \%$ & $6.41 \%$ & 0.000 \\
\hline Impoverished & $0.00 \%$ & $0.00 \%$ & $0.00 \%$ & $0.00 \%$ & $0.00 \%$ & $0.00 \%$ & - & $4.76 \%$ & $2.63 \%$ & $0.00 \%$ & $3.13 \%$ & $0.00 \%$ & $2.19 \%$ & 0.051 \\
\hline \multicolumn{15}{|l|}{ Ataguba method $(y=0.8)$} \\
\hline Catastrophic head count ratio & $9.21 \%$ & $2.35 \%$ & $5.00 \%$ & $0.00 \%$ & $3.85 \%$ & $4.14 \%$ & 0.000 & $20.27 \%$ & $14.29 \%$ & $12.16 \%$ & $9.23 \%$ & $8.16 \%$ & $13.29 \%$ & 0.021 \\
\hline Prepayment poverty head count & $32.88 \%$ & $24.69 \%$ & $20.27 \%$ & $16.92 \%$ & $2.08 \%$ & $20.82 \%$ & 0.000 & $32.88 \%$ & $24.69 \%$ & $20.27 \%$ & $16.92 \%$ & $2.08 \%$ & $20.82 \%$ & 0.000 \\
\hline Post-payment poverty head count & $39.73 \%$ & $25.93 \%$ & $21.62 \%$ & $16.92 \%$ & $4.17 \%$ & $23.17 \%$ & 0.000 & $42.47 \%$ & $28.40 \%$ & $21.62 \%$ & $21.54 \%$ & $4.17 \%$ & $25.22 \%$ & 0.000 \\
\hline Impoverished & $6.85 \%$ & $1.23 \%$ & $1.35 \%$ & $0.00 \%$ & $2.08 \%$ & $2.35 \%$ & - & $9.59 \%$ & $3.70 \%$ & $1.35 \%$ & $4.62 \%$ & $2.08 \%$ & $4.40 \%$ & - \\
\hline \multicolumn{15}{|l|}{ Ataguba method $(y=1)$} \\
\hline Catastrophic head count ratio & $6.67 \%$ & $0.00 \%$ & $2.50 \%$ & $0.00 \%$ & $3.85 \%$ & $2.49 \%$ & 0.000 & $16.44 \%$ & $8.43 \%$ & $8.11 \%$ & $6.15 \%$ & $10.20 \%$ & $9.88 \%$ & 0.005 \\
\hline Prepayment poverty head count & $32.88 \%$ & $24.69 \%$ & $20.27 \%$ & $16.92 \%$ & $2.08 \%$ & $20.82 \%$ & 0.000 & $32.88 \%$ & $24.69 \%$ & $20.27 \%$ & $16.92 \%$ & $2.08 \%$ & $20.82 \%$ & 0.000 \\
\hline Post-payment poverty head count & $39.73 \%$ & $25.93 \%$ & $21.62 \%$ & $16.92 \%$ & $4.17 \%$ & $23.17 \%$ & 0.000 & $42.47 \%$ & $28.40 \%$ & $21.62 \%$ & $21.54 \%$ & $4.17 \%$ & $25.22 \%$ & 0.000 \\
\hline Impoverished & $6.85 \%$ & $1.23 \%$ & $1.35 \%$ & $0.00 \%$ & $2.08 \%$ & $2.35 \%$ & - & $9.59 \%$ & $3.70 \%$ & $1.35 \%$ & $4.62 \%$ & $2.08 \%$ & $4.40 \%$ & - \\
\hline
\end{tabular}

Note: Approach 1 estimates catastrophic health expenditure using health costs only, approach 2 uses health costs plus transport costs. Ataguba method ( $\mathrm{y}=0.8)$ - threshold varies with household expenditure. Ataguba method $(y=1)$ - constant threshold of $10 \%$. 
Table 3-6 Concentration indices for catastrophic health expenditure for diabetes care

\begin{tabular}{|c|c|c|c|c|c|c|}
\hline \multirow{2}{*}{ Indicator } & \multicolumn{3}{|c|}{ Approach 1} & \multicolumn{3}{|c|}{ Approach 2} \\
\hline & $\mathbf{N}$ & Index value & p-value & $\mathbf{N}$ & Index value & $p$-value \\
\hline \multicolumn{7}{|l|}{ WHO Standard method } \\
\hline Catastrophic $10 \%$ & 359 & -0.1246 & 0.000 & 343 & -0.2299 & 0.000 \\
\hline Catastrophic $20 \%$ & 359 & -0.0575 & 0.022 & 343 & -0.1128 & 0.004 \\
\hline Catastrophic 30 \% & 359 & -0.0247 & 0.240 & 343 & -0.0933 & 0.005 \\
\hline Catastrophic $40 \%$ & 359 & -0.0143 & 0.427 & 343 & -0.0744 & 0.015 \\
\hline Impoverished & & & & 320 & -0.0297 & 0.116 \\
\hline \multicolumn{7}{|l|}{ Ataguba method $(y=0.8)$} \\
\hline Catastrophic head count ratio & 362 & -0.0518 & 0.032 & 346 & -0.1026 & 0.015 \\
\hline Impoverished & 341 & -0.0413 & 0.029 & 341 & -0.0512 & 0.046 \\
\hline \multicolumn{7}{|l|}{ Ataguba method $(y=1)$} \\
\hline Catastrophic head count ratio & 361 & -0.0236 & 0.213 & 344 & -0.0582 & 0.118 \\
\hline Impoverished & 341 & -0.0413 & 0.029 & 341 & -0.0512 & 0.046 \\
\hline
\end{tabular}

Note: $\mathrm{N}$ - Number of observations. Note: Approach 1 estimates catastrophic health expenditure using health costs only, approach 2 uses health costs plus transport costs. Ataguba method $(y=0.8)$ - threshold varies with household expenditure. Ataguba method $(y=1)$ - constant threshold of $10 \%$.

\subsubsection{Factors associated with catastrophic health expenditure and impoverishment}

This section analyses the socio-demographic factors associated with catastrophic health expenditure and impoverishment for diabetes care based on results from multivariable logistic regressions. For brevity and due to the loss of statistical power, we present results from the multivariable logistic regression analysis for four out of the twelve estimates of catastrophic health care expenditure, represented here by binary variables. Table 3-7 shows the results for the WHO standard method using a threshold set at $10 \%$ for both approach 1 (medical costs only) and approach 2 (medical and transportation costs). For the Ataguba method, we present results when catastrophic health expenditure for diabetes care is measured using approach 2 (health costs plus transport costs) when the parameter of aversion to inequality is $y=0.8$ and $y=1$. Given the low estimates of catastrophic health expenditure for Ataguba's method when applied using approach 1, regression models were estimated only for approach 2.

Using the WHO method, for approach 1 we see that the factors that increase the odds of catastrophic health expenditure among people with diabetes are being female (Odds ratio [OR] 3.72; Standard error [SE] 2.06), not having children (OR = 4.01; $\mathrm{SE}=2.71)$ and a household 
size of five or more people ( $O R=2.74 ; S E=1.32)$. Patients who were non-African had reduced odds of experiencing catastrophic health expenditure due to diabetes care $(\mathrm{OR}=0.16$; $\mathrm{SE}=$ 0.30). Applying the WHO method to approach 2 , we find that female people with diabetes had increased odds of experiencing catastrophic health expenditure $(\mathrm{OR}=1.73 ; \mathrm{SE}=0.52)$. Falling within the third wealth quintile $(O R=0.49 ; S E=0.20)$, fourth wealth quintile $(O R=$ $0.31 ; \mathrm{SE}=0.15)$ and fifth wealth quintile $(\mathrm{OR}=0.30 ; \mathrm{SE}=0.17)$ wealth quintile were associated with reduced odds of experiencing catastrophic health expenditure. In both the regressions based on the estimates of catastrophic health expenditure calculated with Ataguba's method, people with diabetes who reported not having any children had increased odds of experiencing catastrophic health expenditure for diabetes care $(\mathrm{OR}=3.36$; $\mathrm{SE}=1.83$ when $y=0.8$ and $O R=3.54 ; S E=2.17$ when $y=1)$.

Due to the low incidence of impoverishment when calculated using the WHO method and the loss of statistical power, we present results for the multivariable logistic regressions of impoverishment calculated using the Ataguba method. Using approach 1 (excluding transport costs $)$ patients who were female $(O R=12.83 ; S E=15.94)$ and had no children $(O R=20.38 ; S E$ $=34.50)$ had increased odds of experiencing impoverishment due to diabetes care. Patients who were single $(O R=0.01 ; \mathrm{SE}=0.01)$, within the second $(\mathrm{OR}=0.07 ; \mathrm{SE}=0.11)$ and third $(\mathrm{OR}$ $=0.04 ; \mathrm{SE}=0.06)$ wealth quintiles had reduced odds of experiencing impoverishment due to diabetes health care costs. Using approach 2 (health costs plus transport costs, people with diabetes who reported being single $(O R=0.30 ; S E=0.19)$ had lower odds of impoverishment due to diabetes health care. 
Table 3-7 Factors associated with catastrophic health expenditure for diabetes care

\begin{tabular}{|c|c|c|c|c|c|c|c|c|c|c|c|c|}
\hline \multirow{4}{*}{ Variable } & \multicolumn{8}{|c|}{ Catastrophic health expenditure } & \multirow{2}{*}{\multicolumn{4}{|c|}{$\begin{array}{l}\text { Impoverishment } \\
\text { Ataguba method }\end{array}$}} \\
\hline & \multicolumn{4}{|c|}{ WHO standard method (Threshold = 10\%) } & \multicolumn{4}{|c|}{ Ataguba method } & & & & \\
\hline & \multicolumn{2}{|c|}{ Approach 1} & \multicolumn{2}{|c|}{ Approach 2} & \multicolumn{2}{|c|}{ Approach $2 ; y=0.8$} & \multicolumn{2}{|c|}{ Approach $2 ; y=1$} & \multicolumn{2}{|c|}{ Approach 1} & \multicolumn{2}{|c|}{ Approach 2} \\
\hline & OR & SE & OR & SE & OR & SE & OR & SE & OR & SE & OR & SE \\
\hline Age & 0.99 & 0.0184 & 0.99 & 0.0120 & 1.02 & 0.0158 & 1.01 & 0.0175 & 1.04 & 0.0468 & 0.98 & 0.0248 \\
\hline Female gender & $3.71 * * *$ & 2.0622 & $1.73^{*}$ & 0.5177 & 1.23 & 0.4867 & 2.11 & 1.0001 & $12.82 * *$ & 15.9804 & 2.98 & 2.1404 \\
\hline Non-African & $0.16^{*}$ & 0.1733 & 1.01 & 0.3688 & 1.04 & 0.4895 & 1.43 & 0.7103 & 1 & - & 0.25 & 0.2789 \\
\hline Single marital status & 0.61 & 0.2984 & 0.71 & 0.2097 & 0.88 & 0.3454 & 0.50 & 0.2210 & $0.00 * * *$ & 0.0127 & $0.29 *$ & 0.1900 \\
\hline No children & $4.02 * *$ & 2.7129 & 1.32 & 0.5922 & $3.35^{* *}$ & 1.8273 & $3.54 * *$ & 2.1681 & $20.38 * *$ & 34.5052 & 1.27 & 1.5671 \\
\hline Secondary education & 1.77 & 1.1278 & 0.85 & 0.3249 & 1.08 & 0.5281 & 0.89 & 0.4925 & 3.65 & 4.8304 & 3.11 & 3.5001 \\
\hline Tertiary education & 1.01 & 1.0245 & 0.70 & 0.3958 & 0.82 & 0.6336 & 0.26 & 0.2599 & 7.30 & 16.0224 & 1.23 & 2.0591 \\
\hline Employed & 1.07 & 0.4998 & 0.89 & 0.2709 & 0.63 & 0.2754 & 0.65 & 0.3148 & 0.29 & 0.3094 & 0.42 & 0.3016 \\
\hline $5+$ household size & $2.73 * *$ & 1.3242 & 1.19 & 0.3603 & 1.39 & 0.5499 & 0.88 & 0.4086 & 3.78 & 4.0300 & 2.51 & 1.6182 \\
\hline Index quintile 2 & 0.54 & 0.2913 & 0.75 & 0.2736 & 0.74 & 0.3527 & 0.60 & 0.3349 & $0.07^{*}$ & 0.1077 & 0.57 & 0.4458 \\
\hline Index quintile 3 & 0.47 & 0.2712 & $0.49 *$ & 0.1978 & 0.58 & 0.2981 & 0.50 & 0.3023 & $0.03 * *$ & 0.0617 & 0.19 & 0.2312 \\
\hline Index quintile 4 & 1 & - & $0.30 * *$ & 0.1517 & $0.32 *$ & 0.2153 & 0.37 & 0.2862 & 1 & - & 0.73 & 0.6452 \\
\hline Index quintile 5 & 0.45 & 0.4103 & $0.29 * *$ & 0.1672 & 0.35 & 0.2657 & 0.83 & 0.6068 & 0.21 & 0.4225 & 0.71 & 0.9356 \\
\hline Sample (n) & 256 & & 297 & & 297 & & 295 & & 198 & & 297 & \\
\hline Pseudo $\mathrm{R}^{2}$ & 0.145 & & 0.0574 & & 0.0596 & & 0.0743 & & 0.4151 & & 0.1758 & \\
\hline
\end{tabular}

Notes: Results are for logistic regression models. ${ }^{* *} p<0.01 ;{ }^{* *} p<0.05 ;{ }^{*} p<0.1$, OR - Odds ratio, SE - Standard error. Approach 1 estimates catastrophic health expenditure using health costs only, while approach 2 uses health costs plus transport costs. Ataguba method $(y=0.8)$ - threshold varies with household expenditure. Ataguba method $(y=1)$ constant threshold of $10 \%$. 


\subsection{Discussion}

This study illustrates the burden of diabetes health care amongst patients attending diabetes clinics at subsidised tertiary public health care hospitals in Tshwane, South Africa. To the best of our knowledge it is the first study that assesses the incidence of catastrophic health expenditure amongst people with diabetes and assesses inequalities in catastrophic health expenditure. Our study shows that although considerable progress has been made in health service delivery through for example the provision of free health care when accessing primary health care, failures still exist in making health care affordable for chronic patients within public hospitals. Our findings show that despite the applied UPFS in public sector hospitals, there is a high incidence of catastrophic health care expenditure amongst people with diabetes. To the best of our knowledge there are no previous studies that estimated the incidence of catastrophic health care expenditure amongst people with diabetes in South African public hospitals, we are therefore not able to determine if these inequalities are changing

We find that the existing UPFS has been ineffective in protecting people with diabetes in low socio-economic groups from financial hardship resulting from diabetic health care costs. This finding is consistent with a study that examined health expenditure patterns in rural South Africa and also found high health care costs burdens within poorer wealth quintiles (Goudge et al., 2009). This is of concern given that people with diabetes make multiple visits to health care centres (WHO, 2016a) and have to contend with diabetes related health care costs throughout their life. Although we only focus on indirect costs due to time lost seeking care and time taken off work due to diabetes our finding that indirect costs of diabetes are much higher than the direct costs is consistent with findings from studies presented in Chapter 2 of this dissertation. Our results also show that transport costs contribute over $50 \%$ of the direct diabetes health care costs, which results in much greater levels of catastrophic health expenditure and impoverishment when these costs are considered health care costs. Transport costs have also been found to be high in other health care studies in South Africa (S. Cleary et al., 2013; S. M. Cleary et al., 2012; Goudge et al., 2009). For example, using data from the Agincourt Health and Demographic Surveillance site in South Africa, Goudge et al. (2009) finds that transport costs contribute $42 \%$ to health expenditure (Goudge et al., 2009). 
These high costs point to the urgent need for interventionist programmes to improve access to health care for chronic patients who make multiple visits to health care centres via provision of free or subsidised patient transport services.

Consistent with other studies we find that the incidence of catastrophic health expenditure is sensitive to the method used (Buigut et al., 2015; Kimani et al., 2016; Njagi et al., 2018). Given the arbitrary nature of threshold selection, the high poverty levels amongst Africans in Tshwane (City of Tshwane, 2016), the high transport costs and the high reliance on social grants (30.3\% of households) (South African Institute of Race Relations, 2018), our discussion focuses on the catastrophic health expenditure measured using two methods: (1) health care costs plus transport costs at a $10 \%$ threshold of capacity to pay; (2) health care costs plus transport costs at a variable threshold of total household expenditure. We argue that in this setting, expenditure as low as $10 \%$ of capacity to pay or any expenditure at all is catastrophic.

The incidence of catastrophic health expenditure due to diabetes has previously been investigated in other countries (Gwatidzo \& Williams, 2017; Jing et al., 2018). Using the WHO method and a threshold of $10 \%$, the incidence of catastrophic health expenditure due to diabetes care was $25 \%$. On the other hand, the Ataguba method yields a lower incidence of catastrophic health expenditure due to diabetes of $13 \%$. This finding is similar to other studies that find that the incidence of catastrophic health expenditure is higher when measured as a proportion of capacity to pay (non-food expenditure) compared to when measured as a proportion of total household expenditure (Chuma \& Maina, 2012; Kimani et al., 2016; Qosaj, Froeschl, Berisha, Bellaqa, \& Holle, 2018). The capacity to pay method displays a greater ethical concern for the poor by recognising the higher spending on essential items by poor households when compared to richer households (Hsu et al., 2018; Kimani et al., 2016).

Consistent with results from a systematic review by Njagi et al. (2018), an assessment of the patterns and socioeconomic inequalities in catastrophic health expenditure and impoverishment due to diabetes in our study, showed that the incidence is highest within the lowest socio-economic groups (Njagi et al., 2018). Using a cross sectional sample of 308 people with type 2 diabetes attending a tertiary health care institution in Nigeria, Okoronkwo et al. (2015) also find that the incidence of catastrophic health expenditure is highest within 
the lowest socio-economic group (Okoronkwo et al., 2015). Although we focused on diabetes only, our finding is similar to other studies that find negative $\mathrm{Cls}$ for catastrophic health expenditure (Kien et al., 2017; Kien, Van Minh, Giang, Dao, \& Ng, 2016; Si et al., 2017) and impoverishment (Kien et al., 2017; Kien et al., 2016). This means that catastrophic expenditure due to diabetes health care is concentrated amongst the poor as indicated by the negative $\mathrm{Cl}$ and odds ratios for wealth. To the best of our knowledge there is no study that has investigated inequalities in catastrophic health expenditure due to diabetes in South African public hospitals. We are thus unable to make comparisons with earlier inequality estimates. The results from our study also showed that depending on the method used, up to $4 \%$ experience impoverishment due to diabetes health care. This finding is comparable to other African studies that assessed the impoverishing effects of health care using national datasets (Kwesiga et al., 2015; Mchenga, Chirwa, \& Chiwaula, 2017; Ngcamphalala \& Ataguba, 2018). This means that up to $4 \%$ of households are negatively affected and forced into poverty as a result of diabetes health care costs. This is a huge problem particularly in households that do not only incur diabetes related costs but other health related costs.

Our study also provides insight into the determinants of catastrophic health expenditure and impoverishment amongst people with diabetes visiting public tertiary health care facilities. When measuring catastrophic health expenditure as health care costs plus transport costs at a $10 \%$ threshold of capacity to pay and health care costs plus transport costs at a variable threshold of total household expenditure we found that gender, wealth and children were the variables associated with catastrophic health expenditure. Our finding that households within the higher wealth quintile have reduced odds of incurring catastrophic expenditure, is intuitive and consistent with a study by Babikir et al. (2018) that makes use of the National Income Dynamics Survey (Babikir et al., 2018). Patients who reported not having any children, had increased odds of incurring catastrophic health expenditure and impoverishment. Although the study did not distinguish between young and old or employed and unemployed children, our finding may point to the role that family or social networks play in assisting with health care costs. We found that being female was associated with an increased odds of catastrophic health expenditure and that being single was associated with a reduced odds of impoverishment. We find that when compared to the first quintile the odds of being impoverished for those in the fourth and fifth quintile is statistically insignificant. This finding 
is not consistent with previous literature (Kumar et al., 2015) and calls for further investigations.

\subsection{Limitations}

Our study has some limitations. Due to the recall periods, results may be subject to recall bias. Our study focused on those who used health care services, thus, excluding those with undiagnosed diabetes who still incur health care costs as a result of other illnesses that could be related to diabetes. Our study does not take into account other health care costs that may be incurred by patients and are not related to diabetes. The exclusion of observations with missing data in variables used for the construction of catastrophic health expenditure or impoverishment may have introduced bias in our study results. Also, our study is not nationally representative as it does not reflect the costs associated with diabetes care in private outpatient clinics. The resulting impact is that the findings from this study cannot be generalised as they are not applicable to people with diabetes who access private health care facilities where medical insurance is a common health care payment method. Furthermore, as reflected by the recorded response rate of $81 \%$, the risk of selection bias cannot be ignored. A comparison of our final analytic sample and refusals showed that our final analytic sample contained more Africans (76\% versus $72 \%$ ) and contained less females (61\% versus 62\%). Socio-demographic differences between the final sample and refusals could have introduced bias in the study. The methods used in our study have been criticised for ignoring the role of savings, assets, family and friends in health care financing (Kimani et al., 2016; Rashad \& Sharaf, 2015). Although recent studies encourage the use of a range of equivalence scales when using the WHO method our study only applies one scale. Despite these shortcomings, these methods provide useful measures of catastrophic health expenditure (Kimani et al., 2016) that inform policy makers on the need for financial risk protection amongst people with diabetes visiting public hospitals. Our study may form the basis for future investigations across the public health care facilities in South Africa. Further qualitative research is needed to complement the quantitative findings from this study, in particular to capture the complex nature of factors associated with patients' diabetes management. 


\subsection{Conclusion}

Our results are an important contribution to the literature on diabetes costs amongst patients visiting public hospitals in South Africa. We find that transport costs contribute a significant portion to direct health care costs. These high costs point to the urgent need for interventionist programmes to improve access to health care for chronic patients who make multiple visits to health care centres, such as through subsidised or free transport services. Our study shows that some people with diabetes do incur catastrophic health care expenditure and that inequalities in catastrophic health expenditure favour those within more affluent socio-economic groups. We find that financial protection of diabetes patients visiting South African public hospitals is limited due to the difficulty in identifying unemployed individuals to be exempted from paying for health services. These expenditures are quite high considering the high unemployment rates and multiple annual hospital visits by many of the people with diabetes. Our study also shows that being female and not having children significantly increases the odds of catastrophic health expenditure for diabetes care. Being more affluent reduced the odds of catastrophic health expenditure. These observations suggest health financing interventions amongst people with diabetes should further target the poor and poor women in particular. Thus, a recommendation of the study to government is for the elimination of hospital fees for people with diabetes, which must be accompanied by targeted interventions to reduce transport costs associated with accessing hospitals. 
Chapter 4

Lifestyle and socio-economic inequalities in diabetes prevalence in South Africa: A decomposition analysis

This chapter draws upon:

Mutyambizi C, Booysen F, Stoke A, Pavlova M, Groot W. Lifestyle and socio-economic inequalities in diabetes prevalence in South Africa: A decomposition analysis. PLOS ONE 14 (1): e0211208, 2019 


\section{Abstract}

\section{Background}

Inequalities in diabetes are widespread and are exacerbated by differences in lifestyle. Many studies that have estimated inequalities in diabetes make use of self-reported diabetes which is often biased by differences in access to health care and diabetes awareness. This study adds to this literature by making use of a more objective standardised measure of diabetes in South Africa. The study estimates socio-economic inequalities in undiagnosed diabetes, diagnosed diabetes (self-reported), as well as total diabetes (undiagnosed people with diabetes plus diagnosed people with diabetes). The study also examines the contribution of lifestyle factors to diabetes inequalities in South Africa.

\section{Methods}

This cross-sectional study uses data from the 2012 SANHANES-1 and applies the Erreygers Concentration Indices to assess socio-economic inequalities in diabetes. Contributions of lifestyle factors to inequalities in diabetes are assessed using a decomposition method.

\section{Results}

Self-reported diabetes and total diabetes (undiagnosed people with diabetes plus diagnosed people with diabetes) were significantly concentrated amongst the rich $(\mathrm{Cl}=0.0746 ; p<0.05$ and $\mathrm{Cl}=0.0859 ; p<0.05)$. The $\mathrm{Cl}$ for undiagnosed diabetes was insignificant but pro-poor. The decomposition showed that lifestyle factors contributed $22 \%$ and $35 \%$ to socioeconomic inequalities in self-reported and total diabetes, respectively.

\section{Conclusion}

Diabetes in South Africa is more concentrated amongst higher socio-economic groups when measured using self-reported diabetes or clinical data. Our findings also show that the extent of inequality is worse in the total diabetes outcome (undiagnosed people with diabetes plus diagnosed people with diabetes) when compared to the self-reported diabetes outcome. Although in comparison to other determinants, the contribution of lifestyle factors was modest, these contributions are important in the development of policies that address socioeconomic inequalities in the prevalence of diabetes. 


\subsection{Background}

NCDs are currently the leading cause of death globally. According to the WHO, NCDs are projected to overtake all other causes of death in Africa by the year 2030 (WHO, 2011). In the last two decades, the prevalence of diabetes has increased from $4.7 \%$ in 1980 to $8.5 \%$ of the total world population in 2014 and is expected to further increase especially in LMICs (WHO, 2016a). Between 1990 and 2013, the years of life lost to diabetes globally have increased by 67\% (GBD 2013 Mortality and Causes of Death Collaborators, 2015). Historically diabetes was a burden of developed countries but a huge increase has now been reported in developing countries (WHO, 2016a), countries that often do not have the resources for the prevention, diagnosis, treatment and management of the disease (Kengne, Amoah, \& Mbanya, 2005). In South Africa, the IDF estimates that in 2015, almost 2.3 million people had diabetes (IDF, 2015). The magnitude of the diabetes burden is further reflected in the mortality and causes of death statistics, which show that diabetes has moved from being the fifth leading underlying cause of death in 2013 to being the third and second leading underlying cause of death in 2014 and 2015, respectively (Statistics South Africa, 2017).

Research has been consistent in showing that there is an association between socioeconomic inequalities and diabetes prevalence (Addo et al., 2017; E. Agardh, Allebeck, Hallqvist, Moradi, \& Sidorchuk, 2011; De Silva et al., 2018; Moradi et al., 2016). Whilst earlier studies in high income countries have shown that diabetes prevalence is associated with high socioeconomic status, more recent findings show that it is associated with lower socio-economic status (Addo et al., 2017; E. Agardh et al., 2011). On the other hand, recent findings from middle- and low-income countries show an association of diabetes prevalence with higher socio-economic status (Biswas, Islam, Linton, \& Rawal, 2016; De Silva et al., 2018; Moradi et al., 2016). In Africa, there is a paucity of studies estimating the socioeconomic inequalities in diabetes (Addo et al., 2017), in particular studies that use the $\mathrm{Cl}$ as a measure of inequality. The $\mathrm{Cl}$ is a measure which assesses relative inequality in health. The index shows the distribution of ill-health across the income distribution or some other living standards measure (O'Donnell, Van Doorslaer, Wagstaff, \& Lindelow, 2008). Earlier studies on inequalities in illness in South Africa that have used the $\mathrm{Cl}$ demonstrate that socio-economic inequalities in health exist, however the prevalence across socio-economic status varies by 
disease type (J. E.-O. Ataguba, 2013; J. E. Ataguba, Akazili, \& Mclntyre, 2011; Mukong, Van Walbeek, \& Ross, 2017). Studies that have reported on the socio-economic inequalities in diabetes show that the distribution of illness is higher among more affluent socio-economic groups (J. E. Ataguba et al., 2011; Mukong et al., 2017). Ataguba et al. (2011) find that although the $\mathrm{Cl}$ for diabetes showed that the socio-economic distribution of diabetes was greater among people in higher socio-economic groups the index has declined from 0.10 in 2002 to 0.01 in 2008 (J. E. Ataguba et al., 2011). In a more recent study, Mukong et al. (2017) finds that the $\mathrm{Cl}$ for diabetes was 0.024 in 2008 and 0.034 in 2014-2015 (Mukong et al., 2017). Both studies however relied on self-reported measures of diabetes (J. E. Ataguba et al., 2011; Mukong et al., 2017).

The use of self-reported data on illness without any other standardised measures is reported to result in the exclusion of undiagnosed individuals, especially those in groups with low socioeconomic status (Vellakkal et al., 2015) who might have less access to diagnostic services when compared to high income groups (Manne-Goehler et al., 2016). It is estimated that over two thirds of people with diabetes in the African region are undiagnosed (IDF, 2015). In South Africa, it is estimated that approximately 1.396 million people with diabetes are not diagnosed (IDF, 2015). The magnitude of the unmet need for diabetes care in South Africa has also been previously analysed and reported by Stokes et al. (2017). Using the 2012 SANHANES-1, the authors found that close to half of individuals with diabetes were undiagnosed (Stokes et al., 2017). Poorer and less educated people tend to have worse access to medical care for diabetes diagnosis when compared to the more educated and wealthier individuals (Vellakkal et al., 2015). As a result, the exclusion of undiagnosed people with diabetes may produce biased diabetes prevalence and inequality estimates.

Whilst the causes of type 1 diabetes are unknown, the risk of type 2 diabetes is determined by an interplay of factors such as ethnicity, age, socio-economic status and various lifestyle factors (WHO, 2016a). Lifestyle factors such as unhealthy diets, smoking, alcohol consumption and physical inactivity are particularly important for the prevention of type 2 diabetes (Baliunas et al., 2009; Pan, Wang, Talaei, Hu, \& Wu, 2015; WHO, 2016a), which is more common globally (IDF, 2015; Kengne et al., 2005). The role of modifiable risk factors in explaining the inequality in diabetes has been previously investigated (E. E. Agardh et al., 
2004; Mukong et al., 2017; Stringhini et al., 2012). Health behaviours such as smoking and alcohol consumption explain between $33-45 \%$ of inequalities in the incidence of type 2 diabetes in the United Kingdom (Stringhini et al., 2012) and a third of socioeconomic inequalities in type 2 diabetes in a Swedish based study (E. E. Agardh et al., 2004). Using data from the South Africa National Income Dynamics Survey, Mukong et al. (2017) finds that smoking and alcohol consumption account for $-2.4 \%$ and $2.2 \%$ of self-reported diabetes inequality in 2014-2015 (Mukong et al., 2017). The importance of addressing these risk factors is highlighted in these studies and is also entrenched in the WHO Global Status report on NCDs (WHO, 2014a).

Estimating inequalities in diabetes and determining the contributions of avoidable diabetes risk factors to these inequalities can help South Africa in working towards meeting the 2030 sustainable development goal 3 (SDG 3), which targets a reduction in premature deaths due to NCDs (including diabetes). As mentioned in the introductory chapter, given the high income inequalities in South Africa and their links with health inequalities it is important to assess inequalities in diabetes within the country. This study therefore aims to (1) describe the prevalence, treatment and control of diabetes among South Africans across various socioeconomic groups; (2) to determine socio-economic inequalities in the prevalence of diabetes using the $\mathrm{Cl}$; and (3) to examine the contribution of dietary, lifestyle and metabolic risk factors to socio-economic inequalities in diabetes prevalence by conducting a decomposition analysis. Our study makes important contributions to the body of literature on inequalities in diabetes. To the best of our knowledge this is the first South African study to make use of clinical outcomes in addition to self-reported data in the estimation of socio-economic inequalities in diabetes and the only study that allows a more in-depth analysis into the contribution of a number of lifestyle factors to inequalities in diabetes.

\subsection{Methods}

\subsubsection{Data}

Data are taken from the 2012 SANHANES-1 (Shisana et al., 2014). SANHANES-1 is a nationally representative survey undertaken in April to November 2012 to assess the health and nutrition status of the South African population. It is the first comprehensive national survey 
on NCDs in South Africa. The survey received clearance from the Research Ethics Committee of the HSRC (REC 6/16/11/11). Informed consent was obtained from all study participants. A stratified, multi-stage cluster sample design was employed in sampling the households to be included in the survey. The 2001 population census was used to select a total of 1000 EAs from a database of 86000 EAs. The selection of EAs was stratified by province and locality. In formal urban areas, the selection of EAs was further stratified by race. Based on the master sample of 1000 EAs, a total of 500 EAs were selected based on the socio-demographic profile of South Africa. A random sample of 20 dwellings was then randomly selected from the EAs, yielding a sample of 10000 dwellings or so-called visiting points. Out of the 8168 valid, occupied households (the balance of 1832 dwellings were vacant or could not be located), 6306 households residing at these houses agreed to be interviewed (response rate $=77.2 \%$ ). The dataset includes 26806 individuals.

The SANHANES-1 survey comprised a questionnaire component and a clinical examination. Three questionnaires were administered during the survey: a household questionnaire, an adult questionnaire and a child questionnaire. In this study, the household questionnaire is the source of data for the wealth index. Information on self-reported diabetes and lifestyle factors is drawn from the adult questionnaire. The adult and child questionnaires were administered in the individual's household. The SANHANES-1 survey did not draw a distinction between type 1 and type 2 diabetes.

Blood samples were collected during the clinical examinations which were conducted at various facilities such as school halls, church halls, primary health care facilities, community centres and city halls. The blood samples were collected from individuals, aged 6 years and older, and used for biomarker analysis. The clinical examinations were conducted by experienced medical doctors and nurses on consenting individuals. The blood samples were collected and stored in cooler boxes and delivered to a laboratory within 24 hours. No deviations from established quality control measures were reported.

Our analysis is restricted to individuals above the age of 15 who had a blood sample taken and had non missing information on $\mathrm{HbA1c} ; 17.8 \%$ had missing data on the wealth index and 
were also excluded. The final analytical sample used in this study is 3438 . Details of our exclusion criteria are shown in Figure 4-1.

Figure 4-1 Flow chart for selecting the analytical sample from the general survey sample

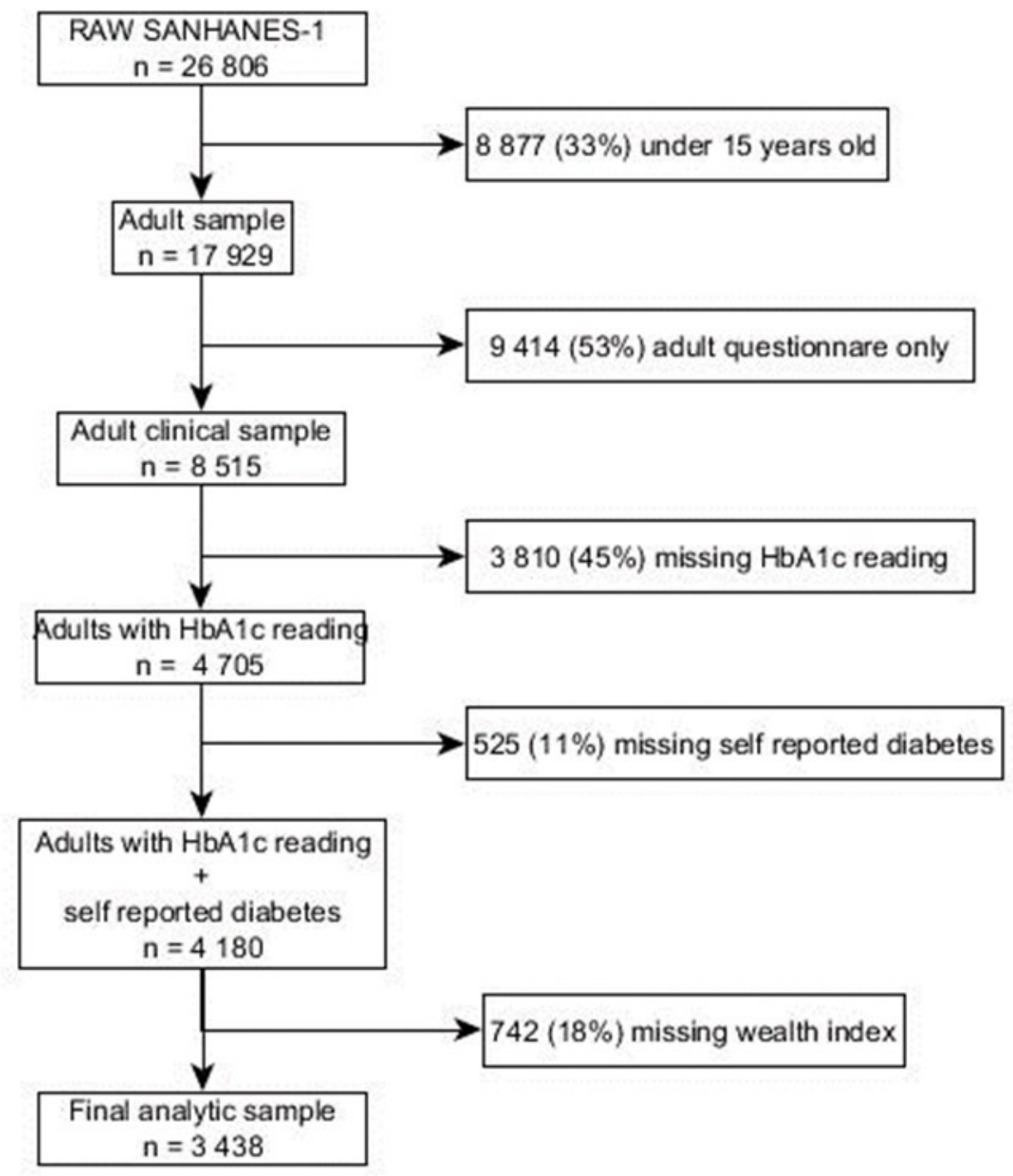

\subsubsection{Measuring inequality}

To measure inequalities in diabetes, this study makes use of the $\mathrm{Cl}$ which was described in Chapter 3. The calculation of the $\mathrm{Cl}$ requires a measure of socio-economic status. In this study, a wealth index is used for this purpose. The wealth index was constructed with the aid of multiple correspondence analysis. The household and living conditions considered in the creation of the wealth index are housing type, water and sanitation services, and a set of thirteen household assets. The full list of thirteen household assets is as follows: ownership 
of a fridge, television, stove, mobile phone, radio, DVD (digital video disc), washing machine, computer, digital satellite television (DSTV), motorcar, vacuum cleaner, telephone (landline) and internet access. Imputation by iterative binomial and multinomial logistic regression analysis, applied using Stata's mi function, is employed to deal with item non-response. Asset ownership is imputed as a function of the ownership of the twelve other assets, whereas housing type is imputed from information on the material of the wall and roof of a dwelling. The percentage inertia explained by the first dimension is approximately $90 \%$.

Consistent with the analysis in Chapter 3 this study makes use of the Erreygers corrected $\mathrm{Cl}$ which is algebraically expressed as shown below (Erreygers, 2009).

$$
E(h)=\frac{4 \mu}{b-a} C I
$$

Where $\mu$ is now the mean of the health variable, $\mathrm{Cl}$ is the standard $\mathrm{Cl}, \mathrm{b}$ is the maximum value of the health variable (in this case 1 ) and a is the minimum value of the health variable (in this case 0). Similar to previous studies (Gonzalo-Almorox \& Urbanos-Garrido, 2016; Makate \& Makate, 2017) we made use of the conindex command in STATA to estimate inequalities.

\subsubsection{Decomposing socio-economic inequality}

The $\mathrm{Cl}$ can be decomposed into the factors that contribute to the measured inequality (Wagstaff, Van Doorslaer, \& Watanabe, 2003). A review of the literature showed that there have been various developments in the methods applied in regression-based decompositions of bivariate inequalities (Erreygers \& Kessels, 2013; Heckley, Gerdtham, \& Kjellsson, 2016; Kessels \& Erreygers, 2016; Wagstaff et al., 2003). Whilst the Wagstaff decomposition technique (Wagstaff et al., 2003) has been the dominant approach this method is one dimensional only (Erreygers \& Kessels, 2013; Heckley et al., 2016). It ignores the correlation between health and the socioeconomic variable but rather focuses on the degree of variation in one variable only (Erreygers \& Kessels, 2013; Heckley et al., 2016). Alternative methods have been suggested in the literature (Erreygers \& Kessels, 2013; Heckley et al., 2016; Kessels \& Erreygers, 2016). For example, Erreygers and Kessels (2013) propose a two dimensional decomposition method that allows an analysis of the two variables (health and income)

simultaneously (Erreygers \& Kessels, 2013). To this Kessels and Erreygers introduced a 
structural equation modelling (SEM) approach which uses different sets of variables to explain the health and socio-economic status variables. Heckley et al. (2016) makes use of the recentered influence function (RIF) regression approach developed by Firpo et al. (2009) to decompose the inequalities into their underlying determinants whilst addressing the limitations within the Wagstaff decomposition method. This approach however relies on a suitable identification strategy. Although such approaches do not ignore the bivariate nature of the bivariate rank dependent indices they have been commented on as being data demanding (Heckley et al., 2016). We adopt the dominant Wagstaff decomposition which also allows comparability with other studies that have used this method within the literature.

Following Wagstaff et al. (2003) our health variable $h_{1}$ (diabetes), is linked to a set of explanatory variable $\mathrm{x}_{\mathrm{ij}}$ by the following linear model.

$$
h_{i}=\alpha+\sum_{j=1}^{q} \beta_{j} x_{i j}+\varepsilon_{i}
$$

If we have such linear model as shown in equation (3) Wagstaff et al. (2003) shows that the $\mathrm{Cl}$ for $h_{1}$ can be written as (Wagstaff et al., 2003):

$$
C I(h)=\sum_{j=1}^{q} \frac{\beta_{j} \overline{x_{j}}}{\mu_{h}} C I\left(x_{j}\right)+\frac{G C_{\varepsilon}}{\mu_{h}}
$$

In equation (4), $\mathrm{Cl}(\mathrm{h})$ is the $\mathrm{Cl}$ for the health variable $\mathrm{h}$ (diabetes), $\overline{x_{j}}$ is the mean of $x_{j}, \mu_{h}$ is the mean of the health variable, $C I\left(x_{j}\right)$ is the $\mathrm{Cl}$ for $x_{j}, G C_{\varepsilon}$ is the generalised $\mathrm{Cl}$ for the error term. In this equation the first part is the weighted sum of the $\mathrm{Cl}$ for the variable $x_{j}$. The weight of each regressor is determined by the elasticity $\left(\beta \overline{x_{j}}\right)$ of h with respect to $x_{j}$. The second part is the residual socio-economic inequalities in health that cannot be explained by the $\mathrm{Cl}$ of the regressors. Since we applied the Erreygers normalisation to the calculation of the $\mathrm{Cl}$ for the socio-economic inequalities in diabetes, the corrected $\mathrm{Cl}$ for the health variable is formulated as:

$$
E(h)=4 \sum_{j=1}^{q} \beta_{j} \overline{x_{j}} C I\left(x_{j}\right)+4 G C_{\varepsilon}
$$


Equation (5) can now be used to decompose socio-economic inequalities in diabetes, showing the contribution of each factor. If the contribution of variable $X$ is positive, then inequality in the health variable would decrease if variable $X$ becomes equally distributed across the socioeconomic group, ceteris paribus. The opposite is also true, if a contribution is negative, the absence of inequalities in that variable would result in an increase in inequality, ceteris paribus.

The absolute contribution a variable makes to socio-economic inequality is a product of the elasticity $\left(\beta \overline{x_{j}}\right)$ of diabetes for each variable and the $\mathrm{Cl}$ for each variable. Therefore, to estimate the contribution, we need to firstly estimate the coefficients of the explanatory variables via a regression. Ordinary Least Squares (OLS), Probit and generalised linear models are the three most common regression methods used for decomposition of inequalities (Yiengprugsawan, Lim, Carmichael, Dear, \& Sleigh, 2010). Yiengprugsawan et al. (2010) compare these three decomposition approaches and show that the use of a generalised linear model (with binomial family and identity link) is the best choice when decomposing inequality of a binary variable (Yiengprugsawan et al., 2010). Since our outcome variable is binary and following Yiengprugsawan et al. (2010) and other studies (Doherty, Walsh, \& O'Neill, 2014; Rarani et al., 2017), this study makes use of the generalised linear model for decomposition of the Erreygers $\mathrm{Cl}$.

As there is no analytical expression for the computation of the standard errors for the contributions generated from equation (4) and since the Stata bootstrap prefix command does not work (J. E. Ataguba, Ojo, \& Ichoku, 2016; O'Donnell et al., 2008), a bootstrapping technique was used to generate the standard errors for the absolute contributions. Whilst taking into account the data's sampling structure we applied the bootstrapping method as described in Efron and Tibshirani (1986) and Efron (1987) and applied in Ataguba et al. (2016). Bootstrapping allows us to assess sampling variability and obtain statistical inference on the results from the decomposition (Guan, 2003). A total of 500 replications were used to estimate the standard errors.

Data analysis was conducted in STATA 13 and post-stratification sample weights were used in all analysis to adjust for unequal probabilities of selection and non-response. 


\subsubsection{Diabetes indicators}

From the analytical sample of 3438 , we identified five main diabetes health indicators: total, undiagnosed, diagnosed, treated and controlled diabetes.

- Total diabetes

Total diabetes was defined as individuals who self-reported being diabetic or had undiagnosed diabetes. The self-reported and undiagnosed diabetes outcomes are explained in more detail below. A total diabetes binary variable was then created to estimate the socioeconomic inequalities in total diabetes (Figure 4-2). The binary variable took on the following values: 0 = individual did not have undiagnosed or self-reported diabetes, 1 = individual selfreported having diabetes or had undiagnosed diabetes.

- Undiagnosed diabetes

Among the sub-sample of total people with diabetes, we calculated the proportion of individuals with undiagnosed diabetes. According to the WHO the diagnostic criteria for diabetes is an $\mathrm{HbA1}$ c level greater than or equal to 6.5\% (WHO, 2016a). Consistent with other studies, we defined diabetes as being undiagnosed when an individual did not self-report prior diabetes diagnosis by a physician, did not report currently taking any diabetic medication, and has a $\mathrm{HbA} 1 \mathrm{c}$ test result of greater than or equal to 6.5\% (Balanda et al., 2013). A binary variable is created to estimate inequalities in undiagnosed diabetes within the total diabetic sample (see Figure 4-2). The binary variable took on the following values: $0=$ individual self-reported being diabetic, 1 = individual had undiagnosed diabetes.

\section{- Diagnosed diabetes}

Based on the SANHANES adult questionnaire, individuals were regarded as having diabetes if they answered yes when asked if a medical doctor or other health care professional had told them that they have high blood sugar or if they answered yes when asked if they are currently taking insulin or tablets to lower their blood sugar levels. A binary variable was then created to estimate the socio-economic inequalities in diagnosed diabetes (see Figure 4-2). The binary variable took on the following values: $0=$ individual did not self-report being diabetic, $1=$ individual self-reported having diabetes or taking diabetic medication. 
- Treated diabetes

Among the diagnosed sample (self-reported people with diabetes), we calculated the proportion of people with diabetes that reported being on diabetic treatment. People with diabetes were considered to be taking treatment if they reported currently taking insulin or tablets to lower their blood glucose levels. A binary variable was created which took on the following values, 0 = if a self-reported individual with diabetes reported not taking insulin or tablets for the lowering of blood glucose levels, and $1=$ if a self-reported individual with diabetes reported taking insulin or tablets for the lowering of blood glucose levels (see Figure $4-2)$.

- Controlled diabetes

Among the sample on diabetes treatment we calculated the proportion of individuals with controlled diabetes. Diabetes was defined as controlled if the respondent reported taking diabetes treatment (insulin or tablets) and had an $\mathrm{HbA} 1 \mathrm{c}$ test of $<6.5 \%$. A binary variable was then created for diabetes control amongst the treated sample, taking on the following values: $0=$ individual was on diabetes treatment and had an $\mathrm{HbA} 1 \mathrm{c}$ test of $>6.5 \%, 1=$ if the individual was on diabetes treatment and had an $\mathrm{HbA1c}$ test of $<6.5 \%$ (see Figure $4-2$ ). 
Figure 4-2 Health care categories sample sizes

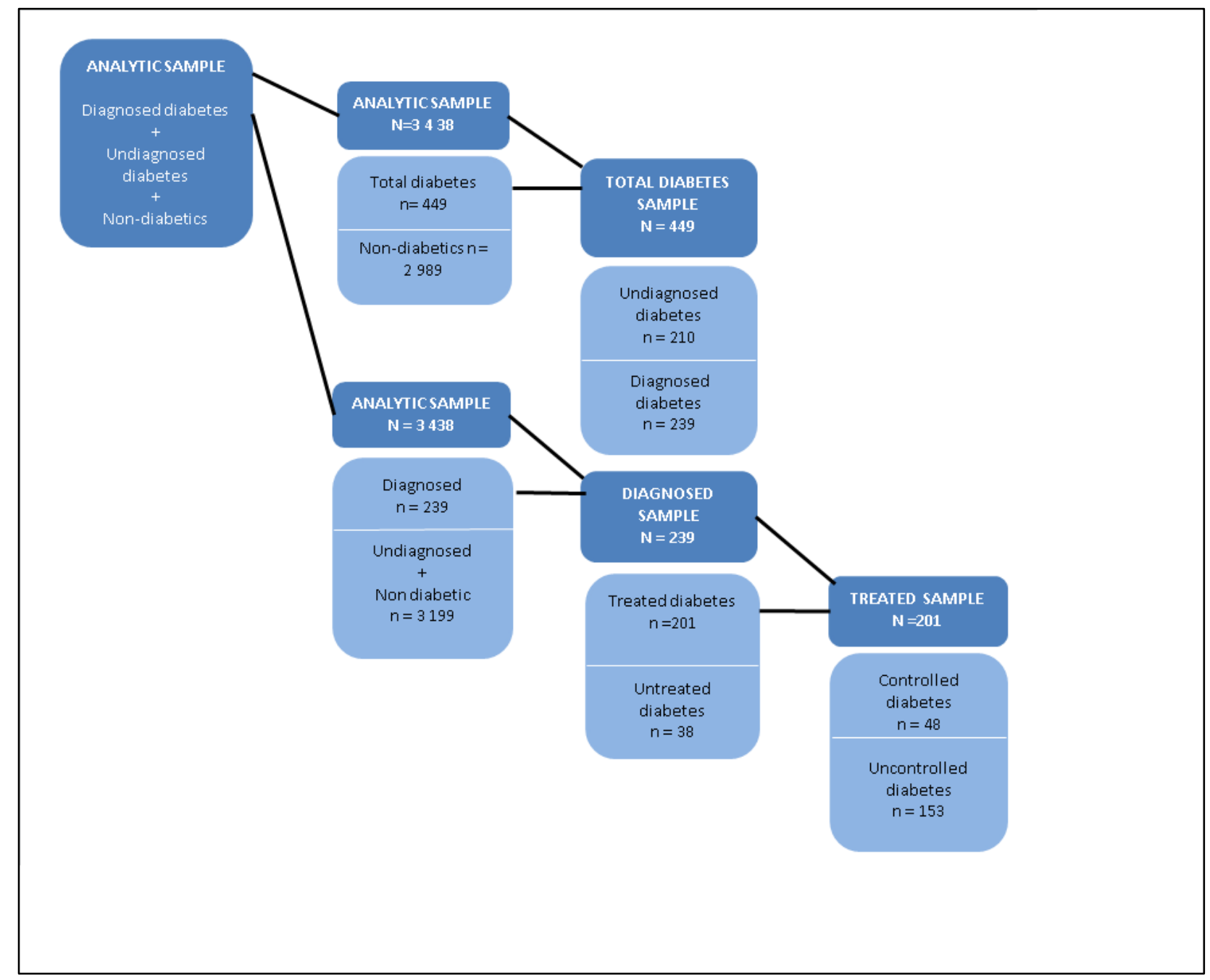

\subsubsection{Explanatory variables - dietary, lifestyle and metabolic risk factors}

A systematic review and meta-analysis by Aune et al. (2015) shows that all types of physical activity are beneficial in reducing the risks of type two diabetes (Aune, Norat, Leitzmann, Tonstad, \& Vatten, 2015). Physical activity data were taken from the Global Physical Activity Questionnaire (GPAQ) within the SANHANES survey. To calculate the intensity of physical exercise, we multiplied weekly activity data of walking, moderate intensity activities and vigorous intensity activities by Metabolic Equivalents (MET) values of 3.3, 4.0 and 8.0 respectively (WHO). The intensity of physical exercise variable (MET-minutes) was then used to create a categorical variable. The WHO recommendations on physical activity is achieving a minimum weekly exercise equivalent to 600 MET-minutes (WHO, 2010b). We categorised the physical activity variable as follows: $0-0 \mathrm{MET}$-minutes, $1->0<600 \mathrm{MET}$-minutes, 2 - >= 
$600<2000$ MET-minutes and 3 - >= 2000 MET-minutes. For unhealthy diet, two measures are used: consumption of fruits and of vegetables. Low fruit and vegetable consumption is referred to as the intake of fewer than five portions a day (WHO, 1990, 2003). Fruit and vegetable consumption is included as categorical variables that took on the values of 0 -none, 1 - less than four times a day, 2 - more than four 4 times a day. Evidence also suggests that smoking is associated with diabetes, however the increase in diabetes risk varies with smoking intensity (Pan et al., 2015). In the SANHANES-1, respondents were also asked how many manufactured cigarettes they smoke per week, this was included as a continuous variable. Because alcohol is reported to have both beneficial and harmful effects, the association of alcohol and the risk of type 2 diabetes are influenced by alcohol drinking frequencies (Baliunas et al., 2009). Alcohol consumption is therefore included as a categorical variable taking the values 0 - never, 1 - occasional and 2 - regularly. Body mass index (BMI) was calculated as weight divided by height squared and included as categorical variable that took on the values, 0 - underweight (BMI< 18.5), 1 - normal weight (BMI $\geq 18.5$ and $<25), 2$ - overweight $(\mathrm{BMI} \geq$ 25 and $<30)$ and $3-$ obese $(\mathrm{BMI} \geq 30)$.

\subsubsection{Other explanatory variables}

Apart from the lifestyle factors and the wealth index, we also included a range of other variables which past literature has shown to influence health (J. E.-O. Ataguba, Day, \& McIntyre, 2015; Obuaku-Igwe, 2015; Solar \& Irwin, 2010). These variables include gender, residence, age, race, employment status, family history of diabetes, insurance and obesity. Gender was included as a binary variable 1 - male, 2 - female. Residence was included as a binary variable with 0 - urban and 1 - rural. Age was measured in years and included as a categorical variable, $15-35$ years, $36-60$ years and $61+$ years. Race was included as a binary variable with 0 - African, 1 - Non-African (i.e. white, coloured and Indian). Employment was included as a binary variable, 0 - unemployed, 1 - employment. Family history of diabetes and insurance were both included as binary variables, 0 - No and $1-$ Yes. 


\subsection{Results}

\subsubsection{Descriptive statistics}

Table 4-1 shows survey weighted descriptive statistics for the study sample according to diabetes outcome. According to the data, the total prevalence rate of diabetes was $11 \%$ (total diabetes). Of the total people with diabetes, $38 \%$ were undiagnosed. The prevalence rate of self-reported diabetes was $7 \%$. Of the self-reported people with diabetes, $61 \%$ were on treatment and $31 \%$ of those on treatment had controlled diabetes.

In each diabetes health outcome category, the sample is predominantly female, resides in urban areas, is unemployed, has no health insurance and is overweight or obese. Our sample is predominantly within the age group of 36 to 60 , with the exception of controlled diabetes. Approximately $63 \%$ of the undiagnosed sample was made up of Africans. The group that selfreported diabetes was predominantly non-African. However, the majority of respondents under treatment and with controlled diabetes were African. Based on the lifestyle factors, our sample predominantly consumed fruits or vegetable portions less than four times a day and did not consume alcohol. A majority of the individuals who self-reported diabetes did not drink alcohol and conducted weekly exercise equivalent to more than 2000 MET-minutes. With the exception of diagnosed diabetes, a majority of the respondents within the health outcomes were obese. 
Table 4-1 Descriptive statistics of variables in study sample

\begin{tabular}{|c|c|c|c|c|c|c|c|c|c|c|}
\hline \multirow{3}{*}{ Variable } & \multicolumn{2}{|c|}{ Total Prevalence } & \multicolumn{2}{|c|}{$\begin{array}{c}\text { Undiagnosed } \\
\text { diabetes }\end{array}$} & \multicolumn{2}{|c|}{$\begin{array}{c}\text { Diagnosed } \\
\text { diabetes }\end{array}$} & \multicolumn{2}{|c|}{ Treated } & \multicolumn{2}{|c|}{ Controlled } \\
\hline & Mean & SE & Mean & SE & Mean & SE & Mean & SE & Mean & SE \\
\hline & \multicolumn{2}{|c|}{$(\mathrm{n}=380 ; 11.09 \%)$} & \multicolumn{2}{|c|}{$(\mathrm{n}=170 ; 38.15 \%)$} & \multicolumn{2}{|c|}{$(n=235 ; 6.86 \%)$} & \multicolumn{2}{|c|}{$(n=145 ; 60.95 \%)$} & \multicolumn{2}{|c|}{$(n=62 ; 31.28 \%)$} \\
\hline \multicolumn{11}{|l|}{ Sex } \\
\hline Male & 0.3296 & 0.0222 & 0.3048 & 0.0318 & 0.3450 & 0.0309 & 0.4409 & 0.0352 & 0.4716 & 0.0728 \\
\hline Female & 0.6704 & 0.0222 & 0.6952 & 0.0318 & 0.6550 & 0.0309 & 0.5591 & 0.0352 & 0.5284 & 0.0728 \\
\hline \multicolumn{11}{|l|}{ Residence } \\
\hline Urban & 0.7775 & 0.0197 & 0.7383 & 0.0304 & 0.8017 & 0.0259 & 0.7491 & 0.0307 & 0.8088 & 0.0574 \\
\hline Rural & 0.2225 & 0.0197 & 0.2617 & 0.0304 & 0.1983 & 0.0259 & 0.2509 & 0.0307 & 0.1912 & 0.0574 \\
\hline \multicolumn{11}{|l|}{ Age category } \\
\hline $15-35$ & 0.1380 & 0.0163 & 0.1922 & 0.0273 & 0.1046 & 0.0199 & 0.0846 & 0.0197 & 0.1183 & 0.0471 \\
\hline $36-60$ & 0.5815 & 0.0233 & 0.5205 & 0.0346 & 0.6191 & 0.0315 & 0.5352 & 0.0354 & 0.3297 & 0.0686 \\
\hline $61+$ & 0.2806 & 0.0212 & 0.2874 & 0.0313 & 0.2764 & 0.0290 & 0.3802 & 0.0344 & 0.5520 & 0.0725 \\
\hline \multicolumn{11}{|l|}{ Race } \\
\hline African & 0.5344 & 0.0236 & 0.6302 & 0.0334 & 0.4753 & 0.0324 & 0.5620 & 0.0352 & 0.5161 & 0.0729 \\
\hline Non-African & 0.4656 & 0.0236 & 0.3698 & 0.0334 & 0.5247 & 0.0324 & 0.4380 & 0.0352 & 0.4839 & 0.0729 \\
\hline \multicolumn{11}{|l|}{ Employment } \\
\hline unemployed & 0.5138 & 0.0239 & 0.5423 & 0.0351 & 0.4962 & 0.0328 & 0.5938 & 0.0351 & 0.6489 & 0.0704 \\
\hline employed & 0.4862 & 0.0239 & 0.4577 & 0.0351 & 0.5038 & 0.0328 & 0.4062 & 0.0351 & 0.3511 & 0.0704 \\
\hline \multicolumn{11}{|l|}{ Diabetes history } \\
\hline No & 0.5463 & 0.0242 & 0.6147 & 0.0345 & 0.5063 & 0.0336 & 0.3525 & 0.0351 & 0.5455 & 0.0768 \\
\hline Yes & 0.4537 & 0.0242 & 0.3853 & 0.0345 & 0.4937 & 0.0336 & 0.6475 & 0.0351 & 0.4545 & 0.0768 \\
\hline \multicolumn{11}{|l|}{ Insurance } \\
\hline No & 0.7280 & 0.0211 & 0.8931 & 0.0215 & 0.6260 & 0.0316 & 0.7526 & 0.0307 & 0.8216 & 0.0564 \\
\hline Yes & 0.2720 & 0.0211 & 0.1069 & 0.0215 & 0.3740 & 0.0316 & 0.2474 & 0.0307 & 0.1784 & 0.0564 \\
\hline \multicolumn{11}{|l|}{ Obesity } \\
\hline underweight & 0.0142 & 0.0058 & 0.0067 & 0.0058 & 0.0190 & 0.0091 & 0.0188 & 0.0099 & 0.0162 & 0.0188 \\
\hline Normal weight & 0.1439 & 0.0171 & 0.1215 & 0.0232 & 0.1582 & 0.0244 & 0.1701 & 0.0275 & 0.2148 & 0.0612 \\
\hline overweight & 0.3802 & 0.0236 & 0.2408 & 0.0304 & 0.4692 & 0.0333 & 0.3259 & 0.0343 & 0.3785 & 0.0723 \\
\hline obese & 0.4616 & 0.0242 & 0.6309 & 0.0343 & 0.3536 & 0.0319 & 0.4852 & 0.0365 & 0.3905 & 0.0727 \\
\hline $\begin{array}{l}\text { Smoking Intensity } \\
\text { (mean) }\end{array}$ & 4.8973 & 0.9055 & 6.5562 & 1.5781 & 4.0890 & 1.1006 & 4.8758 & 1.3973 & 1.9057 & 2.7395 \\
\hline \multicolumn{11}{|l|}{ Alcohol cons } \\
\hline No & 0.8550 & 0.0168 & 0.8578 & 0.0244 & 0.8533 & 0.0232 & 0.8456 & 0.0258 & 0.9346 & 0.0364 \\
\hline Occasional & 0.1118 & 0.0150 & 0.1095 & 0.0218 & 0.1133 & 0.0208 & 0.1072 & 0.0221 & 0.0413 & 0.0293 \\
\hline Regular & 0.0332 & 0.0085 & 0.0327 & 0.0124 & 0.0335 & 0.0118 & 0.0472 & 0.0151 & 0.0241 & 0.0226 \\
\hline Physical activity & & & & & & & & & & \\
\hline None & 0.3510 & 0.0226 & 0.3860 & 0.0337 & 0.3293 & 0.0305 & 0.4102 & 0.0349 & 0.3954 & 0.0713 \\
\hline Low & 0.1047 & 0.0145 & 0.0830 & 0.0191 & 0.1180 & 0.0210 & 0.1423 & 0.0248 & 0.1720 & 0.0550 \\
\hline Moderate & 0.1286 & 0.0158 & 0.1881 & 0.0270 & 0.0919 & 0.0188 & 0.1110 & 0.0223 & 0.1296 & 0.0490 \\
\hline High & 0.4158 & 0.0233 & 0.3429 & 0.0328 & 0.4608 & 0.0324 & 0.3365 & 0.0335 & 0.3030 & 0.0670 \\
\hline $\begin{array}{l}\text { Fruit } \\
\text { consumption }\end{array}$ & & & & & & & & & & \\
\hline None & 0.1092 & 0.0149 & 0.1486 & 0.0250 & 0.0851 & 0.0183 & 0.1177 & 0.0231 & 0.2010 & 0.0591 \\
\hline$<4$ times a day & 0.8415 & 0.0175 & 0.8263 & 0.0266 & 0.8508 & 0.0234 & 0.8031 & 0.0285 & 0.7990 & 0.0591 \\
\hline $4+$ times a day & 0.0493 & 0.0104 & 0.0251 & 0.0110 & 0.0641 & 0.0161 & 0.0793 & 0.0193 & 0.0000 & 0.0000 \\
\hline $\begin{array}{l}\text { Vegetable } \\
\text { consumption }\end{array}$ & & & & & & & & & & \\
\hline None & 0.0335 & 0.0086 & 0.0288 & 0.0117 & 0.0363 & 0.0123 & 0.0594 & 0.0170 & 0.0925 & 0.0427 \\
\hline$<4$ times a day & 0.7557 & 0.0206 & 0.9122 & 0.0198 & 0.6615 & 0.0311 & 0.8297 & 0.0270 & 0.9072 & 0.0428 \\
\hline $4+$ times a day & 0.2108 & 0.0195 & 0.0591 & 0.0165 & 0.3022 & 0.0302 & 0.1109 & 0.0225 & 0.0003 & 0.0026 \\
\hline
\end{tabular}

Note: All estimates are weighted proportions, SE-Standard error 


\subsubsection{Diabetes prevalence across socio-economic groups}

Figure 4-3 shows the distribution of diabetes categories by wealth quintile. From the graph it is clear that the distribution of all diabetes categories is not even across wealth index quintiles. Controlled diabetes was highest in the fourth quintile and all other outcomes were highest in the fifth quintile. All diabetes outcomes were lowest in the first quintile. The number of individuals with undiagnosed diabetes appeared to increase with wealth.

Figure 4-3 Distribution of diabetes by wealth quintile

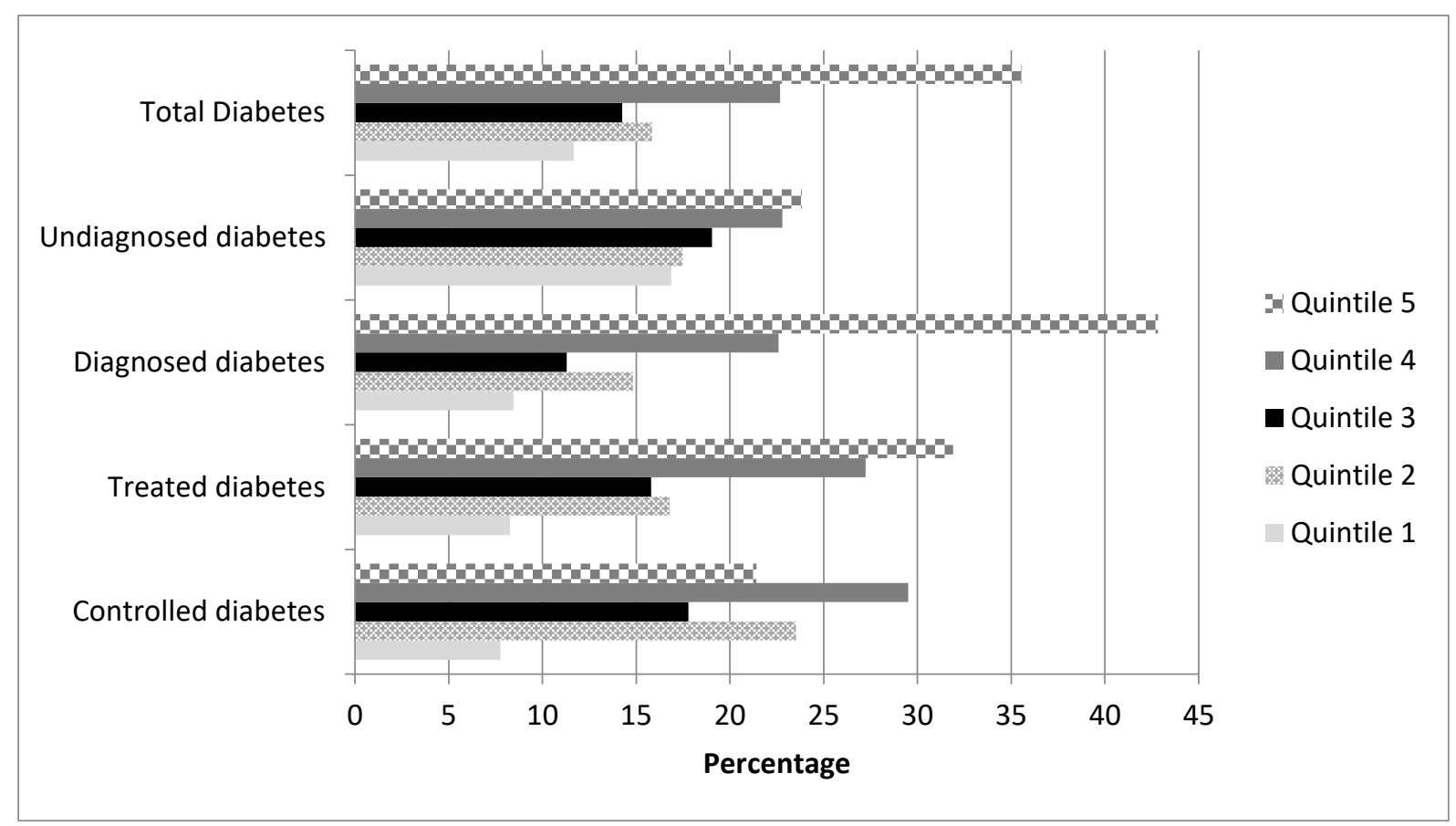

\subsubsection{Lifestyle factors prevalence across socio-economic groups}

Table 4-2 shows that the distribution of lifestyle factors is not even across wealth index quintiles. The majority of respondents who drink alcohol are from the highest wealth quintile. The consumption of fruits and vegetables more than four times a day appears to increase with wealth. The majority of the individuals who do not consume any fruits or vegetables lie within the poorest quintile, whilst the majority that consume fruits and vegetables more than 4 times per week are within the richest quintile. Our results also show that across all wealth quintiles the majority of respondents conducted weekly exercise equivalent to more than 2 
000 MET-minutes. Cigarette consumption intensity was highest within the highest wealth quintile.

Table 4-2 Distribution of lifestyle factor by wealth quintile

\begin{tabular}{lrrrrr}
\hline \multirow{2}{*}{ Variable } & \multicolumn{5}{c}{ Wealth Index Quantiles (\%) } \\
& Poorest & Poor & Middle & Rich & Richest \\
\hline Smoking (mean) & 6.3 & 6.0 & 5.7 & 6.8 & 7.1 \\
Alcohol consumption & & & & & \\
$\quad$ No & 76.0 & 78.6 & 77.2 & 73.4 & 66.7 \\
$\quad$ Occasional & 15.8 & 17.9 & 18.0 & 20.8 & 19. \\
$\quad$ Regular & 8.1 & 3.5 & 4.9 & 5.8 & 14.1 \\
Physical activity & & & & & \\
$\quad$ None & 28.1 & 32.7 & 30.8 & 28.7 & 35.8 \\
$\quad$ Low & 12.2 & 16.0 & 12.8 & 14.3 & 9.0 \\
$\quad$ Moderate & 17.6 & 12.7 & 13.4 & 13.0 & 11.0 \\
$\quad$ High & 42.1 & 38.6 & 43.0 & 44.1 & 44.3 \\
Fruit consumption & & & & & \\
$\quad$ None & 20.4 & 17.1 & 9.8 & 10.0 & 6.6 \\
$\quad<4$ times a day & 74.6 & 80.0 & 87.5 & 83.3 & 83.3 \\
$\quad$ 4+ times a day & 5.0 & 2.9 & 2.7 & 6.8 & 10.1 \\
Vegetable consumption & & & & & \\
$\quad$ None & 9.9 & 7.5 & 3.7 & 6.5 & 2.3 \\
$\quad<4$ times a day & 84.8 & 89.7 & 92.2 & 84.0 & 84.9 \\
4+ times a day & 5.4 & 2.7 & 4.1 & 9.5 & 12.8 \\
\hline Note: All estimates are & & & & &
\end{tabular}

Note: All estimates are weighted

\subsubsection{Concentration Indices}

Table 4-3 shows the corrected Erreygers Cls for each of the three diabetes outcomes. Due to the small sample sizes and loss of statistical power we do not present the Cls for controlled and treated diabetes. The results show that socio-economic inequality is statistically significant for self-reported and total diabetes. All statistically significant Cls are pro-rich, indicating a greater burden of self-reported and total diabetes amongst the higher socioeconomic groups. The extent of inequality is worse in the total diabetes outcome when compared to the self-reported diabetes outcome. The $\mathrm{Cl}$ for undiagnosed diabetes was calculated from a different sub-sample of the data and was pro-poor but not statistically significantly different from zero. 
Table 4-3 Concentration Indices for health categories

\begin{tabular}{lrrr}
\hline Health variable & Erreygers Cl & SE & P-value \\
\hline Self-reported diabetes & 0.0746 & 0.0348 & 0.033 \\
Undiagnosed diabetes & -0.1703 & 0.1257 & 0.177 \\
Total diabetes & 0.0859 & 0.0352 & 0.015 \\
\hline
\end{tabular}

Abbreviation: SE - Standard error, $\mathrm{Cl}$ - Concentration indices

\subsubsection{Decomposition of socio-economic inequality in diabetes}

In order to better understand the lifestyle factors that contribute to inequalities in the diabetes outcomes we conducted a decomposition analysis. The decomposition analysis was conducted only for the measured inequalities in self-reported and total diabetes. Our study does not decompose inequalities in undiagnosed diabetes because our findings show that the measured inequalities in this diabetes outcome was statistically insignificant.

Table 4-4 displays the contribution of lifestyle factors to inequalities in self-reported diabetes and total diabetes. The table shows the margins, the elasticity (product of the coefficient and mean of each explanatory variable), the $\mathrm{Cl}$ of the explanatory variables, the absolute, percentage and total contributions of lifestyle factors whilst also adjusting for other demographic and socio-economics variables. The table also presents the standard errors for the absolute contributions obtained via a bootstrapping method using 500 replications. 
Table 4-4 Decomposition of inequality in self-reported diabetes and total diabetes

\begin{tabular}{|c|c|c|c|c|c|c|c|c|c|c|c|c|c|c|}
\hline \multirow[b]{2}{*}{ Variable } & \multicolumn{7}{|c|}{ Self-reported diabetes } & \multicolumn{7}{|c|}{ Total diabetes } \\
\hline & Margins & Elasticity & $\mathrm{Cl}$ & Absolute & SE & $\%$ & Total & Margins & Elasticity & $\mathrm{Cl}$ & Absolute & SE. & $\%$ & Total \\
\hline \multicolumn{15}{|l|}{ Sex } \\
\hline Male & & (base) & & & & & & & (base) & & & & & \\
\hline Female & -0.0307 & -0.0163 & -0.0205 & 0.0013 & 0.0021 & 1.79 & 1.79 & -0.0392 & -0.0208 & -0.0205 & 0.0017 & 0.0027 & 1.98 & 1.98 \\
\hline \multicolumn{15}{|l|}{ Residence } \\
\hline Urban & & (base) & & & & & & & (base) & & & & & \\
\hline Rural & $0.0481 * *$ & 0.0146 & -0.4458 & -0.0260 & 0.0212 & -34.90 & -34.90 & 0.0275 & 0.0083 & -0.4458 & -0.0149 & 0.0212 & -17.33 & -17.33 \\
\hline $15-35$ & 0.0000 & (base) & & & & & & 0.0000 & (base) & & & & & \\
\hline $36-60$ & $0.1103^{* * *}$ & 0.0411 & 0.0454 & 0.0075 & 0.0076 & 10.00 & & $0.1357^{* * *}$ & 0.0505 & 0.0454 & 0.0092 & 0.0080 & 10.69 & \\
\hline $61+$ & $0.1474 * * *$ & 0.0174 & 0.0987 & 0.0069 & 0.0069 & 9.21 & 19.21 & $0.2107^{* * *}$ & 0.0249 & 0.0987 & 0.0098 & 0.0086 & 11.44 & 22.13 \\
\hline \multicolumn{15}{|l|}{ Race } \\
\hline African & & (base) & & & & & & & (base) & & & & & \\
\hline Non-African & 0.0158 & 0.0043 & 0.4808 & 0.0082 & 0.0183 & 11.05 & 11.05 & $0.0460 *$ & 0.0125 & 0.4808 & 0.0241 & 0.0206 & 27.99 & 27.99 \\
\hline \multicolumn{15}{|l|}{ Wealth index } \\
\hline wealth3 & 0.0561 & 0.0120 & -0.0492 & -0.0024 & 0.0059 & -3.17 & & 0.0362 & 0.0077 & -0.0492 & -0.0015 & 0.0032 & -1.78 & \\
\hline wealth4 & $0.0723 * *$ & 0.0150 & 0.3717 & 0.0222 & 0.0251 & 29.81 & & 0.0622 & 0.0129 & 0.3717 & 0.0192 & 0.0162 & 22.29 & \\
\hline wealth5 & $0.0784 * *$ & 0.0165 & 0.7893 & 0.0522 & 0.0376 & 69.91 & 65.77 & 0.0428 & 0.0090 & 0.7893 & 0.0285 & 0.0253 & 33.11 & 27.10 \\
\hline $\begin{array}{l}\text { Employment } \\
\text { unemployed }\end{array}$ & & (base) & & & & & & & (base) & & & & & \\
\hline employed & 0.0000 & 0.0000 & 0.1406 & 0.0000 & 0.0051 & 0.01 & 0.01 & 0.0118 & 0.0044 & 0.1406 & 0.0025 & 0.0054 & 2.86 & 2.86 \\
\hline \multicolumn{15}{|l|}{ Diabetes history } \\
\hline No & & (base) & & & & & & & (base) & & & & & \\
\hline Yes & $0.0719 * * *$ & 0.0178 & 0.1388 & 0.0099 & 0.0043 & 13.23 & 13.23 & $0.0668 * * *$ & 0.0165 & 0.1388 & 0.0092 & 0.0045 & 10.67 & 10.67 \\
\hline \multicolumn{15}{|l|}{ Insurance } \\
\hline No & & (base) & & & & & & & (base) & & & & & \\
\hline Yes & 0.0155 & 0.0028 & 0.6177 & 0.0068 & 0.0119 & 9.11 & 9.11 & -0.0319 & -0.0057 & 0.6177 & -0.0140 & 0.0174 & -16.27 & -16.27 \\
\hline
\end{tabular}




\begin{tabular}{|c|c|c|c|c|c|c|c|c|c|c|c|c|c|c|}
\hline \multirow[b]{2}{*}{ Variable } & \multicolumn{7}{|c|}{ Self-reported diabetes } & \multicolumn{7}{|c|}{ Total diabetes } \\
\hline & Margins & Elasticity & $\mathrm{Cl}$ & Absolute & SE & $\%$ & Total & Margins & Elasticity & $\mathrm{Cl}$ & Absolute & SE. & $\%$ & Total \\
\hline \multicolumn{15}{|l|}{ Obesity } \\
\hline underweight & & (base) & & & & & & & (base) & & & & & \\
\hline normal weight & -0.0270 & -0.0110 & -0.1356 & 0.0060 & 0.0233 & 8.01 & & 0.0019 & 0.0008 & -0.1356 & -0.0004 & 0.0276 & -0.48 & \\
\hline overweight & 0.0252 & 0.0063 & 0.1260 & 0.0032 & 0.0137 & 4.27 & & $0.08702 * *$ & 0.0219 & 0.1260 & 0.0110 & 0.0183 & 12.82 & \\
\hline obese & $0.0639 *$ & 0.0173 & 0.1354 & 0.0094 & 0.0249 & 12.55 & 24.83 & $0.1357^{* * *}$ & 0.0367 & 0.1354 & 0.0199 & 0.0288 & 23.16 & 35.49 \\
\hline \multicolumn{15}{|l|}{ Alcohol cons } \\
\hline No & & (base) & & & & & & & (base) & & & & & \\
\hline Occasional & -0.0274 & -0.0050 & 0.0630 & -0.0013 & 0.0014 & -1.70 & & $-0.0579 * * *$ & -0.0106 & 0.0630 & -0.0027 & 0.0024 & -3.12 & \\
\hline Regular & $-0.1123 * * *$ & -0.0083 & 0.1766 & -0.0059 & 0.0049 & -7.89 & -9.59 & $-0.1397 * * *$ & -0.0104 & 0.1766 & -0.0073 & 0.0050 & -8.53 & -11.65 \\
\hline \multicolumn{15}{|l|}{ Physical activity } \\
\hline None & & (base) & & & & & & & (base) & & & & & \\
\hline Low & -0.0402 & -0.0051 & -0.0405 & 0.0008 & 0.0027 & 1.11 & & -0.0140 & -0.0018 & -0.0405 & 0.0003 & 0.0018 & 0.34 & \\
\hline High & 0.0061 & 0.0026 & 0.0059 & 0.0001 & 0.0015 & 0.08 & 0.35 & -0.0001 & 0.0000 & 0.0059 & 0.0000 & 0.0018 & 0.00 & 0.07 \\
\hline \multicolumn{15}{|l|}{ Fruit consumption } \\
\hline None & & (base) & & & & & & & (base) & & & & & \\
\hline$<4$ times a day & 0.0311 & 0.0255 & 0.0212 & 0.0022 & 0.0056 & 2.90 & & 0.0260 & 0.0213 & 0.0212 & 0.0018 & 0.0091 & 2.10 & \\
\hline $4+$ times a day & -0.0056 & -0.0003 & 0.1927 & -0.0002 & 0.0099 & -0.33 & 2.57 & -0.0426 & -0.0024 & 0.1927 & -0.0018 & 0.0129 & -2.14 & -0.04 \\
\hline \multicolumn{15}{|c|}{ Vegetable consumption } \\
\hline None & & (base) & & & & & & & (base) & & & & & \\
\hline$<4$ times a day & $-0.0632 *$ & -0.0551 & -0.0027 & 0.0006 & 0.0026 & 0.78 & & -0.0199 & -0.0174 & -0.0027 & 0.0002 & 0.0026 & 0.21 & \\
\hline $4+$ times a day & 0.0525 & 0.0037 & 0.2250 & 0.0033 & 0.0076 & 4.47 & 5.25 & $0.1684 * * *$ & 0.0119 & 0.2250 & 0.0107 & 0.0053 & 12.45 & 12.67 \\
\hline \multicolumn{7}{|c|}{ Dietary, lifestyle and metabolic risk factors } & 22.24 & & & & & & & 34.67 \\
\hline Total observed & & & & 0.0803 & & & & & & & 0.0809 & & & \\
\hline Residual & & & & -0.0057 & & & & & & & 0.0050 & & & \\
\hline Total & & & & 0.0746 & & & & & & & 0.0859 & & & \\
\hline
\end{tabular}


As shown in Table 4-4, demographic and socio-economics variables that were significantly associated with self-reported diabetes were living in rural areas $(p<=0.05)$, increasing age $(p<=0.01)$, wealth $(p<=0.05)$ and family history of diabetes $(p<=0.01)$. Factors significantly associated with total diabetes were increasing age $(p<=0.01)$, being non-African $(p<=0.1)$, wealth quintile $2(p<=0.1$ ) and a family history of diabetes $(p<=0.01)$. Factors that contributed the most to socioeconomic inequalities in both self-reported and total diabetes were residence (urban or rural dwelling), wealth index (socio-economic status) and age. Our results show that residence explains $-34.9 \%$ of the inequality in self-reported diabetes and $-17.3 \%$ of the inequality in total diabetes. Thus, if inequalities in diabetes were determined by this variable alone they would favour the better off. According to Table 4-4 the wealth index is also a significant contributor to self-reported diabetes (65.77 \%) and total diabetes $(27.10 \%)$. The contribution of the wealth index to diabetes inequalities is higher for self-reported diabetes compared to total diabetes because of the different elasticity values. Age category was also another large contributor to inequality, contributing $19.2 \%$ to inequalities in selfreported diabetes and $22.1 \%$ to inequalities in total diabetes. Race and family history of diabetes also make notable contributions to inequality.

The marginal effects in Table 4-4 show that the lifestyle factors significantly associated with self-reported diabetes were being obese $(p<=0.1)$, regular alcohol consumption $(p<=0.01)$ and vegetable consumption less than four times a day $(p<=0.1)$. Factors significantly associated with total diabetes were obesity $(p<=0.05)$, smoking $(p<=0.1)$, alcohol consumption $(p<=0.01)$ and vegetable consumption more than four times a day $(p<=0.01)$. Results from the decomposition show that lifestyle factors contributed a total of $22.2 \%$ and $34.7 \%$ to inequalities in self-reported and total diabetes respectively. Among the lifestyle factors, obesity, alcohol and vegetable consumption made the largest contribution to diabetes inequalities (see Table 4-4). Obesity contributed approximately $24.8 \%$ to inequalities in selfreported diabetes and $35.5 \%$ to inequalities in total diabetes. In the absence of inequalities in obesity, inequalities in diabetes would decrease. Vegetable consumption is another important contributor to diabetes inequalities, $5.3 \%$ for self-reported diabetes and $12.7 \%$ for total diabetes. The positive contribution by vegetable consumption indicates that if vegetable consumption was equally distributed across the wealth index then inequalities in selfreported diabetes would decrease by $5.3 \%$ and $12.7 \%$ for total diabetes. Alcohol consumption 
contributed $-9.6 \%$ to inequalities in self-reported diabetes and $-11.7 \%$ to inequalities in total diabetes, meaning that if alcohol consumption was distributed equally amongst the population, inequalities in self-reported and total diabetes would increase. Smoking intensity, fruit consumption and physical activity made marginal contributions to diabetes inequalities. The residuals in Table 4-4 represent the unexplained sources of inequalities.

\subsection{Discussion}

Our paper provides evidence on the socio-economic inequalities in various diabetes outcomes using the $\mathrm{Cl}$ and identifies the contribution of lifestyle factors to socio-economic inequalities in diabetes prevalence by conducting a decomposition analysis. To the best of our knowledge this is the first study to incorporate biomarker analysis in the measurement of diabetes inequalities in South Africa and the first to attempt to measure the contribution of various lifestyle factors to socio-economic related inequalities in diabetes. Consistent with the study by Stokes et al. (2017), our study documents the high levels of undiagnosed diabetes in South Africa (Stokes et al., 2017). This study showed that the total prevalence of diabetes in South Africa was $11 \%$, of which $38 \%$ were undiagnosed. The poor rates of diagnosis are largely a result of insufficient access to health care and poor health care systems (Manne-Goehler et al., 2016). The prevalence of self-reported diabetes was $6.86 \%$ of which $61 \%$ were on treatment and $31 \%$ of those on treatment had controlled diabetes. The poor rates of treatment and control have also been previously reported by Stokes et al. (2017) and have been attributed to poor diabetes education and medication adherence (Manne-Goehler et al., 2016; Stokes et al., 2017).

Our findings corroborate other related literature that demonstrates the existence of socioeconomic inequalities in diabetes (J. E. Ataguba et al., 2011; Mukong et al., 2017). Furthermore, consistent with previous studies that estimated inequalities in self-reported diabetes in South Africa our study finds that the prevalence of self-reported diabetes is prorich (J. E. Ataguba et al., 2011; Mukong et al., 2017). Although our findings on the inequality in undiagnosed diabetes were not statistically significantly different from zero, we find that the size of the inequality in diagnosed diabetes was further intensified by the inclusion of undiagnosed people with diabetes. This finding informs the development of studies that seek 
to produce more robust inequality estimates of NCD prevalence in South Africa. The finding also contributes to international literature that has attempted to use the $\mathrm{Cl}$ to compare the use of self-reported diagnosis versus standardised measures of diagnosis in the estimation of socioeconomic inequalities in health (Vellakkal et al., 2015; Vellakkal et al., 2013). Although the NCDs considered in these studies excluded diabetes, the direction of inequality between self-reported chronic diseases and standardised measures of chronic disease diagnosis showed a mixed picture that varied by disease type and country (Vellakkal et al., 2015; Vellakkal et al., 2013).

The decomposition of inequalities has become an important tool in influencing policy in inequality studies. Decompositions provide important information on the sources of the observed inequalities. Whilst the largest contributions to the inequalities in diabetes in this study came from residence and socio-economics status, the contributions of lifestyle factors further exacerbate these inequalities and are the focus of this analysis. Various studies have attempted to estimate the contribution of lifestyle factors to health in general (Borg \& Kristensen, 2000; McGrail, Van Doorslaer, Ross, \& Sanmartin, 2009; Mukong et al., 2017; Vallejo-Torres \& Morris, 2010) and diabetes specifically (E. E. Agardh et al., 2004; Stringhini et al., 2012; Williams et al., 2010). A study by Borg and Kristensen (2000) showed that lifestyle factors and work environment contribute approximately two thirds to the social gradient in self-reported health (Borg \& Kristensen, 2000). Our study shows that lifestyle factors contributed a total of $22.2 \%$ and $34.7 \%$ to inequalities in self-reported and total diabetes, respectively. Previous studies suggest that these factors explain between $33-45 \%$ of inequalities in the incidence of type 2 diabetes in the United Kingdom (Stringhini et al., 2012), a third of socioeconomic inequalities in type 2 diabetes in a Swedish based study (E. E. Agardh et al., 2004) and $27 \%$ when estimated using the Australian Diabetes Obesity and Lifestyle Study (Williams et al., 2010).

Amongst the lifestyle factors in our study obesity makes the largest contribution to socioeconomic inequalities in both self-reported and total diabetes variables. Stringhini et al. (2012), using data from the London Whitehall II Study also finds that amongst the health behaviours in the study obesity was the most important contributor to the relationship between socio-economic status and diabetes (Stringhini et al., 2012). Obesity is widely 
regarded as a risk factor for ill health (WHO, 2014a) and type 2 diabetes (Guh et al., 2009; WHO, 2016a). Consistent with a study by Alaba and Chola (2014), using the South African National Income Dynamics Study (NIDS), we observe a pro-rich distribution of disparities in obesity (Alaba \& Chola, 2014). Our findings show a much larger contribution of obesity to selfreported and total diabetes $(24.8 \%$ and $35.5 \%)$ than the contribution of obesity to social inequalities in health reported in the London Whitehall II study (18\% - 20\%\%) (Vallejo-Torres \& Morris, 2010). South Africa is reported to be undergoing an epidemic of overweight and obesity that is closely linked to nutrition changes (Sartorius et al., 2017), severely impacting health outcomes.

Although evidence suggests that diets rich in fruits and vegetable are associated with a reduced risk of type 2 diabetes (Carter, Gray, Troughton, Khunti, \& Davies, 2010; Du et al., 2017; Li, Fan, Zhang, Hou, \& Tang, 2014), the mechanisms through which fruits and vegetables consumption influences the diabetes risk is not well established (Carter et al., 2010). Whilst some studies showed that the consumption of fresh fruit (Du et al., 2017) was associated with a reduction in the risk of type 2 diabetes other literature shows that the reduction in risk is related to the fruit or vegetables sub-types consumed (Muraki et al., 2013). In particular dietary fibre is reported to regulate insulin which helps reduce diabetes risk (Bazzano $A$. $L$, 2005) and green leafy vegetables are inversely related to diabetes (Carter et al., 2010; Cooper et al., 2012). The observed differences are likely a result of the use of food frequency questionnaires rather than biomarkers (such as vitamin C) (Carter et al., 2010). In our study vegetable consumption was associated with diabetes prevalence and contributed more to socio-economic inequalities in diabetes when compared to fruit consumption. Consistent with previous literature we find that lower consumption of fruits and vegetables is concentrated amongst those within low socio-economic groups (Hosseinpoor et al., 2012; Kunna, San Sebastian, \& Williams, 2017). In South Africa factors such as urban migration and globalisation are reported to be the cause of the nutrition transition that has resulted in the consumption of energy-dense foods and sugary beverages (Sartorius et al., 2017).

Findings within the literature on the association between alcohol and diabetes have not been consistent (Baliunas et al., 2009). Some studies report a protective effect at moderate consumption levels (Baliunas et al., 2009; Carlsson, Hammar, Grill, \& Kaprio, 2003), an 
increased risk at high consumption (Carlsson et al., 2003), a protective effect even at high consumption (Conigrave et al., 2001) and other studies find that the risk of diabetes in high alcohol consumers is the same as in abstainers (Carlsson et al., 2003). Our study finds that regular and occasional alcohol consumption was negatively correlated with diabetes. Overall the rates of regular or occasional alcohol consumption in our study are quite low (25\%), people with diabetes in our sample were less likely to drink alcohol. Alcohol consumption is reported to have both beneficial and harmful effects on health. Thus, the impact of alcohol consumption on health is a function of the length, volume, patterns and type of alcohol consumed. In our study alcohol made one of the largest contributions to inequalities in diabetes amongst the lifestyle factors. Contrary to other studies we find much larger contributions of alcohol to inequalities in diabetes (Mukong et al., 2017; Stringhini et al., 2012).

In our study smoking and physical activity make the smallest contributions to inequalities in diabetes. This is a result of the very small marginal effects and elasticities. Similar to the existing literature (Mukong et al., 2017), we also found no evidence that smoking contributes significantly to inequalities in diabetes, while contrary to the literature, we did not find any evidence that physical activity explains a substantive proportion of inequalities in diabetes ( $E$. E. Agardh et al., 2004). These differences most probably are attributable to differences in behavioural and situational contexts in different countries and settings.

\subsection{Limitations}

The study has some limitations that must be acknowledged. Despite its clinical convenience, the $\mathrm{HbA1c}$ test used in the SANHANES-1 survey is reported to have several limitations (George, 2011). A major limitation of the test is that the optimal cut-off of $6.5 \%$ as recommended by ADA, may not be optimal for some ethnic groups. Other factors such as age, HIV status and anaemia are also reported to affect $\mathrm{HbA} 1$ levels. Studies across Africa and in South Africa have shown that the test is not equivalent to the oral glucose tolerance test as a screening tool for diabetes (George, 2011; Zemlin, Matsha, Hassan, \& Erasmus, 2011). Thus, the use of the $\mathrm{HbA1c}$ test as a diabetes diagnosis tool in our study, may have resulted in an overestimation or underestimation of diabetes prevalence. Whilst there are several 
regression-based decomposition methods within the literature our study makes use of the Wagstaff method. Results may differ depending on the decomposition method applied (Heckley et al., 2016). The ADA states that although the risk of developing diabetes increases with age, there is no exact age for the onset of type 1 or type 2 diabetes, thus we were unable to separate type 2 from type 1 people with diabetes (American Diabetes Association, 2015). Despite this, lifestyle factors such as alcohol, physical activity and fruit consumption which are common risk factors for type 2 diabetes were included in our analysis as explanatory variables. Another limitation of the study is the low number of individuals who went to the testing centres and provided a blood sample. Our analytical sample may be prone to selfselection of individuals that went to get blood samples taken as well as those who completed the adult and household questionnaires. We therefore compared our final analytical sample to the 2011 South African census across sex, age, race and province. Compared to the 2011 census our analytic sample contained a larger sample of non-Africans ( $27 \%$ versus 23 ) and a smaller sample of Africans ( $72 \%$ versus $77 \%$ ). Our sample also contained fewer individuals within the age category of 15 to 35 years (51\% versus 55\%). The analytical sample employed in this study therefore is not nationally representative, which means caution is necessary in drawing generalisations from the empirical results. It is also possible that our self-reported data on lifestyle factors suffered from social desirability bias. For example, an under reporting of smoking patterns or alcohol consumption could potentially influence the contributions made by these factors to diabetes inequalities.

\subsection{Conclusion}

This chapter provides an analysis of the socio-economic inequalities in the prevalence of diabetes and determines the sources of these inequalities with a focus on modifiable lifestyle factors. The chapter contributes to the literature on diabetes by making use of a more objective measure of diabetes and highlighting the magnitude of undiagnosed diabetes in South Africa. The study provides evidence that inequality in self-reported and total diabetes is concentrated among the rich. The magnitude of inequality estimates based on self-reported data only would be different when compared to inequality estimates based both on selfreported plus clinical data. The measured inequalities are mostly explained by residence and wealth. The contributions made by lifestyle factors to inequalities in diabetes, are less than the overall contributions of other factors within our model. Although modest, the 
contributions made by lifestyle factors to inequalities in diabetes provide important information for use in planning of interventions to reduce the burden of diabetes. Our study shows that in comparison to all other lifestyle factors obesity, alcohol consumption and vegetable consumption make large contributions to inequalities in diabetes. These findings are important to policy makers in terms of informing the design of effective strategies and policies for encouraging healthy lifestyles. Future national health surveillance surveys that capture larger numbers of individuals who provide blood samples are an ideal conduit for the monitoring of diabetes and the tracking of socio-economics inequalities in the prevalence, diagnosis and treatment of diabetes. 
Chapter 5

Inequalities and factors associated with adherence to diabetes self-care practices amongst patients at two public hospitals in Gauteng, South Africa

This chapter draws upon:

Mutyambizi C, Pavlova M, Hongoro C, Groot W. Inequalities and factors associated with adherence to diabetes self-care practices amongst patients at two public hospitals in Gauteng, South Africa. BMC Endocrine Disorders. 2020;20(1):15 


\section{Abstract}

\section{Background}

Self- management is vital to the control of diabetes. This study aims to assess the diabetes self-care behaviours of patients attending two tertiary hospitals in Gauteng, South Africa. The study also seeks to estimate the inequalities in adherence to diabetes self-care practices and associated factors.

\section{Methods}

This study makes use of data collected at two public hospitals in 2017. Diabetes selfmanagement practices considered in this study are dietary diversity, medication adherence, physical activity, self-monitoring of blood-glucose, avoiding smoking and limited alcohol consumption. Concentration indices (Cls) were used to estimate inequalities in adherence to diabetes self-care practices. Multiple logistic regressions were fitted to determine factors associated with diabetes self-care practices.

\section{Results}

Approximately $99 \%$ of the sample did not consume alcohol or consumed alcohol moderately, $92 \%$ adhered to self-monitoring of blood-glucose, $85 \%$ did not smoke tobacco, $67 \%$ adhered to their medication, $62 \%$ had a diverse diet and $9 \%$ adhered to physical activity. Self-care practices of dietary diversity $(\mathrm{Cl}=0.1512)$ and exercise $(\mathrm{Cl}=0.1067)$ were all concentrated amongst patients with higher socio-economic status as indicated by the positive Cls, whilst not smoking $(\mathrm{Cl}=-0.0994)$ was concentrated amongst those of lower socio-economic status as indicated by the negative $\mathrm{Cl}$. Dietary diversity was associated with being female, being retired and higher wealth index. Medication adherence was found to be associated with older age groups. Physical activity was found to be associated with tertiary education, being a student and those within higher wealth index. Self-monitoring of blood glucose was associated with being married. Not smoking was associated with being female and being retired. 


\section{Conclusion}

Adherence to exercising, dietary diversity and medication was found to be sub-optimal. Dietary diversity and exercise were more prevalent among patients with higher socioeconomic status. Our findings suggest that efforts to improve self- management should focus on addressing socio-economic inequalities. It is critical to develop strategies that help those within low-socio-economic groups to adopt healthier diabetes self-care practices. 


\subsection{Background}

Diabetes Mellitus is a serious and common chronic illness globally, and a major cause of limb amputations, blindness, kidney failure and stroke (WHO, 2016a). It is reported that people living with diabetes are at an increased risk of developing additional health problems and infections when compared to people without diabetes (IDF, 2015). The risk of CVDs in people living with diabetes is double that for people not living with diabetes (Emerging Risk Factors Collaboration, 2010). Furthermore, diabetes is associated with an excess risk of mortality from several non-vascular conditions such as cancer (Emerging Risk Factors Collaboration, 2011). Diabetes and its complications are a major cause of mortality globally. The IDF estimates that globally 463 million (9.3\%) people had diabetes and that diabetes and its complications were the cause of over 4 million deaths amongst people aged 20-79 years old in 2019 (IDF, 2019). South Africa is reported to have the highest prevalence of diabetes in the African region (12.7\% in 2019) and the highest number of deaths due to diabetes among LMICs in 2019 (89 800 deaths) (IDF, 2019).

The morbidity of diabetes is related to its diabetes-related complications and multimorbidity, which is associated with poor glycaemic control (American Diabetes Association, 2017). In South Africa, poor glycaemic control has been reported in hospital-based studies conducted across the country (Erasmus, Blanco, Okesina, Gqweta, \& Matsha, 1999; Govender, Gathiram, \& Panajatovic, 2017; Nthangeni et al., 2002; Pinchevsky, Butkow, Chirwa, \& Raal, 2017; Shilubane, 2010). Furthermore, using the nationally representative SANHANES-1, Stokes et al. (2017) shows that among individuals with diabetes, $18.1 \%$ were treated but uncontrolled (had an $\mathrm{HbA1c}$ greater than or equal to 7\%) (Stokes et al., 2017). According to the ADA and the SEMDSA, a glycaemic level equal to or below $7 \%$ is considered optimum (American Diabetes Association, 2017; SEMDSA Type 2 Diabetes Guidelines Expert Committee, 2017). Achieving this level of diabetes control is an outcome of a complex mix of both pharmacological and non-pharmacological management practices (WHO, 2016a). Thus, diabetes management requires actions by different role-players (such as patients, their families and health care providers) to ensure improved outcomes. Whilst pharmacological management consists of the use of medicines (oral hypoglycaemic agents and or insulin therapy), non-pharmacological 
management involves person education and support in the adoption of diabetes self-care practices (WHO, 2016a).

Based on international studies the SEMDSA developed guidelines that are used for the management of diabetes in South Africa (Amod \& Berg, 2012; SEMDSA Type 2 Diabetes Guidelines Expert Committee, 2017). As emphasised by the guidelines, adherence to diabetes self-care is an integral part of diabetes management, contributing to improved glycaemic levels, reduced development of diabetes complications and associated costs, and improved quality of life (American Diabetes Association, 2017). The essential components of diabetes self-care include for example healthy eating, physical activity, tobacco smoking cessation, weight management, medication adherence, self-monitoring of blood glucose levels, blood pressure and feet, routine screening of eye and renal complications (American Diabetes Association, 2017; IDF, 2017). Adherence to these self-care practices is influenced by a variety of factors such as socio-economic status, diabetes education, health beliefs, education level, family history of diabetes and patient demographic characteristics (Gurmu, Gela, \& Aga, 2018; Kassahun, Gesesew, Mwanri, \& Eshetie, 2016; Luo et al., 2015; Mogre, Abanga, Tzelepis, Johnson, \& Paul, 2017).

A systematic review by Stephani et al. (2018) shows that the levels of adherence to diabetes self-care practices in Sub-Saharan Africa are poor and a threat to achieving improved health outcomes (Stephani, Opoku, \& Beran, 2018). In South Africa, studies that have investigated patient management of diabetes have been limited to using qualitative methods to investigate the challenges people living with diabetes face in the management of the chronic illness (Adeniyi, Yogeswaran, Wright, \& Longo-Mbenza, 2015; Masupe, Ndayi, Tsolekile, Delobelle, \& Puoane, 2018; Mendenhall \& Norris, 2015; Murphy, Chuma, Mathews, Steyn, \& Levitt, 2015; Steyl \& Phillips, 2014). Studies that used quantitative methods investigated the prevalence or distribution of dietary (Nthangeni et al., 2002) and exercise practices amongst people with diabetes (Okonta, Ikombele, \& Ogunbanjo, 2014). One study that assessed the factors associated with diabetes self-care practices focused on medication adherence only (Adegbola, Marincowitz, Govender, \& Ogunbanjo, 2016). Our study expands on these previous studies and assesses adherence to diabetes self-care practices amongst people with diabetes visiting two tertiary hospitals in Gauteng, South Africa. A study of this nature is 
important for facilitating actions for improved diabetes self-care practices amongst people living with diabetes, particularly those from low resource settings, such as those visiting public hospitals. Our study therefore aims to: (1) describe adherence to the following diabetes selfcare behaviours: dietary diversity, medication adherence, physical activity, self-monitoring of blood-glucose, avoiding smoking and limited alcohol consumption; (2) estimate the inequalities in these self-care behaviours using Concentration Indices (Cls); (3) describe the association between adherence to diabetes self-care behaviours and patients' demographic characteristics.

\section{$5.2 \quad$ Methods}

\subsubsection{Study setting}

Similar to Chapter 3 this chapter makes use of data collected from two tertiary hospitals in Tshwane. Patients referred to the clinics usually have diabetic complications or poorly controlled blood sugar. The diabetes clinics at the hospitals are open on specific clinic days during the week and operate on a structured consultation schedule. Health education at the hospitals is provided by a health education team consisting of the nurses, medical doctors and dieticians. Patients are scheduled to attend the clinic every three months and at each consultation a different focus is set such as foot examination, eye examination or dietician consultation.

\subsubsection{The questionnaire}

As mentioned in Chapter 3 the questionnaire development was guided by the SANHANES-1 data collection tool and adapted to the South African public hospital context. In addition to the diabetes related costs, income and expenditure questions used for the study in Chapter 3 the questionnaire also consists of a food frequency questionnaire, the Global Physical Activity Questionnaire (QPAQ), questions related to alcohol, tobacco use, self-monitoring of blood glucose and the Morisky medication adherence instrument. The questionnaire is easy to administer and practical to use with illiterate populations and the elderly. The questionnaire was pretested with 8 patients at one of the hospitals to ensure validity and 
reliability. Amendments were then made were necessary. Given that the primary objective of the study was to collect information related to household expenditure and income, data collection was restricted to individuals above the age of 21 years. No incentives or inducement was offered to participate in the study. Quality checks of all completed questionnaires was done by the data collection supervisor at the end of each data collection day.

\subsubsection{Inequalities in diabetes self-care behaviours}

To determine the inequalities in diabetes self-care behaviours, our study makes use of the widely employed $\mathrm{Cl}$ which was also used and explained in Chapter 3. In this study, to determine the wealth categories, we make use of the wealth index calculated in Chapter 3.

\subsubsection{Study variables and analysis}

Dietary diversity: A healthy diet is an essential part of diabetes management. Because one single food cannot be the source of all required nutrients, consuming a varied diet increases the likelihood of consuming all required nutrients (Labadarios, Steyn, \& Nel, 2011). Dietary diversity scores (DDSs) are an indication of diet nutrient adequacy (Kennedy, 2009). Therefore, to assess diet quality our study makes use of the DDS. DDS is defined as the number of food groups consumed by an individual over a 24 hour period (Hatløy, Torheim, \& Oshaug, 1998). Survey respondents were asked to recall the foods they ate the day before the interview. These food items were then linked to corresponding food groups and these groups were used to calculate the DDS. Our study followed methods developed in other studies in the estimation of DDSs (Steyn \& Nel, 2006; Steyn, Nel, Nantel, Kennedy, \& Labadarios, 2006). The nine food groups were: cereals, roots and tubers; vitamin-A-rich vegetables and fruits; other fruit; other vegetables; meat, poultry and fish; legumes; fats and oils; dairy products and eggs. A nine-point scale was then created using the number of food groups consumed by the individual. Dietary inadequacies are associated with a DDS below 4 (Kennedy, 2009; Steyn \& Nel, 2006; Steyn et al., 2006). Consistent with other studies, the operational definition for DDS adherence in this study is respondents who have a DDS greater than or equal to 4 
(Labadarios et al., 2011). Thus, a binary variable was created that took a value of one when DDS was $>=4$ and otherwise, it took a value of zero.

Medication: The level of medication adherence was measured using the Morisky's instrument - a validated four-question preformed questionnaire (Morisky, Green, \& Levine, 1986). Consistent with other studies that have applied the Morisky's instrument, individuals were considered adherent to medication if they gave negative responses to all four questions (Sharma, Kalra, Dhasmana, \& Basera, 2014). Medication adherence was included as a binary variable which took on a value of one if patients adhered to medication and otherwise, it took a value of zero.

Physical activity: The WHO recommends at least 150 minutes of moderate intensity physical activity and 75 minutes of vigorous intensity physical activity per week (WHO, 2010b). In our study, individuals were considered adherent to physical activity if they reported taking part in more than two hours of any of the following physical activities per week: bicycling, brisk walking/jogging, sport activities, strength exercises, aerobic exercises and any other exercises.

Self-monitoring of blood-glucose: Although self-monitoring of blood-glucose is recommended for people with diabetes, the IDF and SEMDSA advise that the frequency of self-monitoring of blood-glucose is dependent on individual clinical needs (IDF, 2009; SEMDSA Type 2 Diabetes Guidelines Expert Committee, 2017). The SEMDSA guidelines recommend those using insulin to test at least once a day and for those using oral medication testing 3-5 times per week may be sufficient. Our operational definition for adherence to self-monitoring of blood-glucose is conducting a glucose test everyday over the past week for patients on insulin and at least 3 times per week when using tablets (SEMDSA Type 2 Diabetes Guidelines Expert Committee, 2017).

Smoking status: In this study, individuals were asked to report if they currently smoked tobacco. The operational definition for non-smoking-related adherence was a respondent who reported not currently smoking tobacco. A binary variable was created for non-smoking- 
related adherence which took on a value of 1 when individuals did not smoke and otherwise it took on a value of zero.

Alcohol consumption: Individuals were asked to report how often they consumed alcohol in the last 12 months. This variable was included as a binary variable that took on a value of one if the individual reported never consuming alcohol or consuming alcohol up to four times a month and a value of zero when individuals reported consuming alcohol more than 4 times a week.

Socio-demographic characteristics: Our study also included the following variables: age category (21-40 years, 41-60 years and 60+ years), sex, race (African/non-African), marital status (single, married, divorced, widowed and co-habiting), having children (yes/no), education level (primary, secondary, tertiary), employment status (unemployed, formally employed, informally employed, student and retired), household size (1-4 members/5+ members) and wealth index quintile (quintile 1 , quintile 2 , quintile 3 , quintile 4 and quintile 5).

Statistical analysis was conducted in STATA 13. First descriptive analysis was performed. Then, multivariable Logistic regression analysis was conducted to assess the factors associated with each of the diabetes self-care behaviours. Thus, we have separate regressions for each selfcare behaviour.

\subsection{Results}

Out of the 503 patients who were invited to take part in the survey, 405 patients agreed to be interviewed. Due to incomplete data, 9 patients were excluded from the analysis. Thus our study sample included 396 diabetes patients.

\subsubsection{Descriptive statistics}

Table 5-1 shows the socio-demographic characteristics of the study sample. The majority of our study sample was between the age of 41 and 60 years. Approximately $61 \%$ were female, 
$76 \%$ were African, $35 \%$ were single, $86 \%$ reported having children, $66 \%$ had secondary education, 49\% were unemployed and 64\% came from households with 1 to 4 members.

Table 5-1 Socio-demographic characteristics of people with diabetes $(\mathrm{N}=396)$

\begin{tabular}{|c|c|c|}
\hline Variable & Frequency & Percent \\
\hline \multicolumn{3}{|l|}{ Age category } \\
\hline $21-40$ & 98 & 25.4 \\
\hline $41-60$ & 171 & 44.3 \\
\hline $60+$ & 117 & 30.3 \\
\hline \multicolumn{3}{|l|}{ Sex } \\
\hline Male & 155 & 39.2 \\
\hline Female & 240 & 60.8 \\
\hline \multicolumn{3}{|l|}{ Race } \\
\hline African & 300 & 76.3 \\
\hline Non-African & 93 & 23.7 \\
\hline \multicolumn{3}{|l|}{ Marital status } \\
\hline Single & 136 & $35 \%$ \\
\hline Married & 162 & $41 \%$ \\
\hline Divorced & 36 & $9 \%$ \\
\hline Widowed & 52 & $13 \%$ \\
\hline Co-habiting & 7 & $2 \%$ \\
\hline \multicolumn{3}{|l|}{ Children } \\
\hline Yes & 337 & 86.0 \\
\hline No & 55 & 14.0 \\
\hline \multicolumn{3}{|l|}{ Education } \\
\hline Primary & 65 & 16.8 \\
\hline Secondary & 255 & 65.7 \\
\hline Tertiary & 68 & 17.5 \\
\hline \multicolumn{3}{|c|}{ Employment status } \\
\hline Unemployed & 184 & $49 \%$ \\
\hline Formal & 107 & $29 \%$ \\
\hline Informal & 30 & $8 \%$ \\
\hline Student & 8 & $2 \%$ \\
\hline Retired & 44 & $12 \%$ \\
\hline \multicolumn{3}{|l|}{ Household size } \\
\hline $1-4$ & 249 & 63.7 \\
\hline $5+$ & 142 & 36.3 \\
\hline
\end{tabular}




\subsubsection{Diabetes self-care behaviours amongst people living with diabetes}

Table 5-2 shows the distribution of diabetes self-care practices within our study sample. The majority of the participants were adherent to limited alcohol consumption (98.72\%). Approximately $92.18 \%$ of the study sample reported to adhere to self-monitoring of bloodglucose. In addition to the results in Table 5-2, the majority of the study participants reported having a machine to monitor blood glucose (92\%). Of those who did not adhere to selfmonitoring of blood-glucose, $8 \%$ reported not having a machine to measure blood glucose. Table 5-2 also shows that non-smoking adherence was also common at $85.3 \%$. Approximately $66.84 \%$ adhered to their diabetes medication regime and $62.27 \%$ had dietary diversity. Over $90 \%$ of the study sample did not adhere to physical activity. Being too sick to exercise was the most commonly reported reason for non-adherence to exercise.

Table 5-2 Diabetes self-care behaviours amongst people with diabetes

\begin{tabular}{lrr}
\hline Variable & Frequency & Percent \\
\hline Limited alcohol consumption (N=391) & 5 & \\
$\quad$ No & 386 & 98.7 \\
$\quad$ Yes & & \\
SMBG (N=358) & 28 & 7.8 \\
$\quad$ No & 330 & 92.2 \\
$\quad$ Yes & & \\
Non-smoking (N=381) & 56 & 14.7 \\
$\quad$ No & 325 & 85.3 \\
Yes & & \\
Medication Adherence (N=374) & 124 & 33.2 \\
$\quad$ No & 250 & 66.8 \\
$\quad$ Yes & & \\
Dietary diversity (N=387) & 146 & 37.7 \\
$\quad$ No & 241 & 62.3 \\
Yes & & \\
Exercise (N=396) & 359 & 90.7 \\
$\quad$ No & 37 & 9.3 \\
$\quad$ Yes & & \\
\hline
\end{tabular}

Table 5-3 shows the distribution of self-care behaviour by wealth index quintile. Adherence to exercise and self-monitoring of blood glucose appeared to increase by wealth quintile whilst adherence to non-smoking decreased by wealth quintile. The majority of individuals who adhered to dietary diversity, belong to the fifth quintile. In all wealth quintiles, the 
majority of individuals adhered to their diabetic medication and limited alcohol consumption. In addition, this study found that a majority of the study participants adhered to three out of the six diabetes self-care behaviours (42\%). This was followed by those who adhered to four out of the six diabetes health care behaviours (34\%). Approximately $18 \%$ adhered to two out of the six diabetes self-care behaviours, $4 \%$ adhered to five and $1 \%$ adhered to none.

Table 5-3 Distribution of self-care behaviour by wealth quintile

\begin{tabular}{lrrrrrr}
\hline \multirow{2}{*}{ Variable } & \multicolumn{7}{c}{ Wealth Index quintiles (\%) } \\
& $\mathbf{1}$ & $\mathbf{2}$ & $\mathbf{3}$ & $\mathbf{4}$ & $\mathbf{5}$ & Overall \\
\hline Dietary diversity & $47 \%$ & $65 \%$ & $67 \%$ & $64 \%$ & $71 \%$ & $62 \%$ \\
Medication adherence & $74 \%$ & $72 \%$ & $57 \%$ & $63 \%$ & $67 \%$ & $67 \%$ \\
Exercise & $4 \%$ & $7 \%$ & $11 \%$ & $11 \%$ & $17 \%$ & $9 \%$ \\
SMBG & $90 \%$ & $92 \%$ & $92 \%$ & $93 \%$ & $95 \%$ & $92 \%$ \\
Non-Smoking & $91 \%$ & $88 \%$ & $83 \%$ & $83 \%$ & $81 \%$ & $85 \%$ \\
Limited alcohol consumption & $97 \%$ & $99 \%$ & $99 \%$ & $100 \%$ & $98 \%$ & $99 \%$ \\
\hline
\end{tabular}

\subsubsection{Inequalities in diabetes self-care behaviours}

Table 5-4 shows the Cls for diabetes self-care behaviours, namely dietary diversity, medication adherence, physical activity, self-monitoring of blood-glucose and non-smoking status. Due to the small sample sizes and loss of statistical power, we do not present results for inequalities in limited alcohol consumption. Whilst the Cls for dietary diversity, physical exercise and non-smoking were statistically significant, the Cls for medication adherence and self-monitoring of blood-glucose were all statistically insignificant. From the table, dietary diversity and physical exercise were all concentrated amongst the rich participants whilst nonsmoking was concentrated amongst the poor participants.

Table 5-4 Inequalities in diabetes self-care behaviours

\begin{tabular}{lcrrrrr}
\hline Variable & Obs & Index & \multicolumn{2}{c}{$\begin{array}{c}\text { 95\% Confidence } \\
\text { interval }\end{array}$} & SE & P-value \\
\hline Dietary diversity & 387 & 0.1512 & 0.0405 & 0.2619 & 0.0565 & 0.008 \\
Medication adherence & 374 & -0.0909 & -0.2009 & 0.0190 & 0.0561 & 0.106 \\
Exercise & 396 & 0.1067 & 0.0413 & 0.1722 & 0.0334 & 0.002 \\
SMBG & 358 & 0.0275 & -0.0368 & 0.0918 & 0.0328 & 0.402 \\
Non-Smoking & 381 & -0.0994 & -0.1810 & -0.0178 & 0.0416 & 0.018 \\
\hline
\end{tabular}

Notes: Obs - Observations, SE - Standard Error, Cl - Concentration Index 


\subsubsection{Factors associated with diabetes self-care behaviours}

The results of the five separate multivariable logistic regressions for each of the self-care behaviours, namely dietary diversity, medication adherence, physical activity, self-monitoring of blood-glucose and non-smoking status are presented in Table 5-5. Below, the key findings are summarized.

Dietary diversity: The variables significantly associated with dietary diversity were being female (Odds Ratio [OR] 1.60; Standard error [SE] 0.41) versus being male, retired $(2.33 ; 1.13)$ versus unemployed, wealth quintile $2(2.87 ; 1.01)$, wealth quintile $3(2.41 ; 0.88)$, wealth quintile $4(2.46 ; 0.97)$ and wealth quintile $5(4.65 ; 2.15)$ versus wealth quintile 1 (lowest wealth).

Medication adherence: The variables significantly associated with medication adherence were age category $41-60$ years $(2.63 ; 0.91)$, age category $60+$ years $(6.37 ; 3.26)$ versus age category of $21-40$ years, being non-African $(0.35 ; 0.12)$ versus being African, widowed (0.34; $0.17)$ versus being single, wealth quintile $2(0.34 ; 0.14)$ versus wealth quintile 1

Physical exercise: The variables significantly associated with physical exercise were tertiary education $(6.39 ; 7.18)$ versus primary education, being a student $(6.00 ; 5.67)$ versus being unemployed, household size $>5(0.43 ; 0.20)$ versus household size $<5$, wealth quintile 4 (3.66; $2.79)$ and wealth quintile $5(5.15 ; 4.12)$ versus wealth quintile 1

Self-monitoring of blood-glucose: The variables significantly associated with self-monitoring of blood-glucose were age category of $61+$ years $(0.26 ; 0.22)$ versus age category of $21-40$ years, and being non-African $(0.25 ; 0.14)$ versus being African, being married $(2.93 ; 1.86)$ versus being single.

Non-Smoking: The variables significantly associated with not smoking were being female (7.69; 3.13) versus being male and being non-African $(0.26 ; 0.11)$ versus being African, being retired $(4.23 ; 3.26)$ versus being unemployed. Thus, female, African or retired people living with diabetes in our study had higher odds to adhere to non-smoking compared to male, nonAfrican or unemployed respondents whose odds to adhere to non-smoking were lower. 
Table 5-5 Factors associated with diabetes self-care behaviours (multivariable logistic regressions)

\begin{tabular}{|c|c|c|c|c|c|c|c|c|c|c|}
\hline \multirow{2}{*}{ Variable } & \multicolumn{2}{|c|}{ Dietary diversity } & \multicolumn{2}{|c|}{ Medication adherence } & \multicolumn{2}{|c|}{ Exercise } & \multicolumn{2}{|c|}{ SMBG } & \multicolumn{2}{|c|}{ Non-Smoking } \\
\hline & OR & SE & OR & SE & OR & SE & OR & SE & OR & SE \\
\hline Age 41-60 & 0.81 & 0.2650 & $2.63^{* * *}$ & 0.9112 & 1.00 & 0.5289 & 0.68 & 0.4510 & 1.41 & 0.6725 \\
\hline Age 60+ & 0.76 & 0.3343 & $6.38 * * *$ & 3.2640 & 0.36 & 0.3091 & $0.27^{*}$ & 0.2274 & 0.72 & 0.4675 \\
\hline Female & $1.61^{*}$ & 0.4134 & 1.14 & 0.3174 & 1.04 & 0.4417 & 0.42 & 0.2463 & $7.69 * * *$ & 3.1335 \\
\hline Non-African & 0.81 & 0.2589 & $0.36 * * *$ & 0.1206 & 0.52 & 0.2743 & $0.25^{* *}$ & 0.1499 & $0.27 * * *$ & 0.1130 \\
\hline Married & 1.14 & 0.3493 & 1.14 & 0.3842 & 1.07 & 0.5521 & $2.93^{*}$ & 1.8681 & 1.51 & 0.7080 \\
\hline Divorced & 1.24 & 0.5959 & 0.83 & 0.4247 & 0.77 & 0.6072 & 5.28 & 5.9890 & 0.74 & 0.4607 \\
\hline Widowed & 0.78 & 0.3494 & $0.35 * *$ & 0.1705 & 0.85 & 0.7111 & 1.22 & 0.8426 & 2.14 & 1.7505 \\
\hline Co-habiting & 1.30 & 1.1209 & 0.71 & 0.6763 & 1.06 & 1.3268 & 1.00 & (empty) & 1.23 & 1.4795 \\
\hline No Children & 0.75 & 0.2888 & 0.80 & 0.3158 & 0.74 & 0.4442 & 0.55 & 0.3869 & 1.25 & 0.6677 \\
\hline Secondary education & 1.44 & 0.4926 & 0.89 & 0.3604 & 4.49 & 4.7725 & 0.64 & 0.4694 & 0.44 & 0.3025 \\
\hline Tertiary education & 1.39 & 0.6451 & 0.96 & 0.4877 & $6.39 *$ & 7.1806 & 0.38 & 0.3572 & 0.41 & 0.3236 \\
\hline Formal employment & 1.12 & 0.3388 & 0.86 & 0.2774 & 2.10 & 0.9704 & 0.93 & 0.5893 & 1.82 & 0.8490 \\
\hline Informal employment & 0.89 & 0.4177 & 0.95 & 0.4837 & 1.00 & (empty) & 1.39 & 1.6196 & 0.71 & 0.4361 \\
\hline Student & 1.07 & 0.9883 & 0.75 & 0.6576 & $6.00^{*}$ & 5.6769 & 1.00 & (empty) & 1.00 & (empty) \\
\hline Retired & $2.33^{*}$ & 1.1345 & 1.37 & 0.7383 & 3.24 & 2.5307 & 0.49 & 0.3454 & $4.23^{*}$ & 3.2636 \\
\hline Household size 5+ & 1.24 & 0.3253 & 0.83 & 0.2378 & $0.44^{*}$ & 0.2036 & 0.49 & 0.2506 & 1.22 & 0.4990 \\
\hline Wealth Quintile 2 & $2.87^{* * *}$ & 1.0212 & 1.07 & 0.4295 & 1.69 & 1.2799 & 2.93 & 2.0445 & 0.98 & 0.5778 \\
\hline Wealth Quintile 3 & $2.41^{* * *}$ & 0.8814 & $0.34 * * *$ & 0.1436 & 2.86 & 2.1307 & 2.11 & 1.4218 & 0.54 & 0.3121 \\
\hline Wealth Quintile 4 & $2.47^{* *}$ & 0.9726 & 0.50 & 0.2178 & $3.66^{*}$ & 2.7972 & 4.02 & 3.4623 & 1.10 & 0.6825 \\
\hline Wealth Quintile 5 & $4.65^{* * *}$ & 2.1532 & 0.99 & 0.4739 & $5.15^{* *}$ & 4.1241 & 4.10 & 3.6775 & 0.79 & 0.5055 \\
\hline Sample (n) & 334 & & 324 & & 316 & & 298 & & 322 & \\
\hline Pseudo $\mathrm{R}^{2}$ & 0.0604 & & 0.1141 & & 0.1156 & & 0.1471 & & 0.2189 & \\
\hline
\end{tabular}

Notes: OR - Odds Ratio, SE - Standard Error, Reference categories: Age 21-40 years, male, African, single, having children, primary education, unemployed, household size 1-4 and wealth quintile 1 (lowest wealth). All outcome variables are binary in which a value of 1 indicates a diverse diet, medication adherence, exercise, self-monitoring of blood glucose and smoking. 


\subsection{Discussion}

Diabetes is a serious chronic illness that leads to the development of complications and early mortality if not controlled and managed. In this chapter, we examined diabetes self-care management practices of patients attending two tertiary hospitals in Gauteng, South Africa. Our analysis focused on six self-care practices of dietary diversity, medication adherence, physical activity, self-monitoring of blood-glucose, non-smoking status and limited alcohol consumption. An assessment of adherence to diabetes self-care practices and their inequalities together with the identification of factors associated with these behaviours, is important for the design of strategies to control diabetes. Our findings for each diabetes selfcare practice are discussed below.

Although a variety of dietary approaches (such as low carbohydrate diets or low fat diets) have been applied in the management of diabetes, there has not been one single diet that has been identified as being superior to the rest in diabetes management (SEMDSA Type 2 Diabetes Guidelines Expert Committee, 2017). However, it is acknowledged that a diet with varied nutrients is associated with improved diabetes management outcomes (SEMDSA Type 2 Diabetes Guidelines Expert Committee, 2017). A diet that lacks diversity is often an indication of food scarcity, which is often associated with malnutrition (Labadarios et al., 2011). Our hospital-based study revealed that approximately $38 \%$ of our study sample had poor dietary diversity (DDS<4). Using a nationally representative South African dataset, a study by Labadarios et al. (2011) showed that at the national level, approximately $38 \%$ have poor dietary diversity. Our findings are lower than those reported among Ethiopian type 2 diabetes patients in a hospital-based study which found that $76 \%$ of patients did not adhere to recommended diet (Bonger, Shiferaw, \& Tariku, 2018). They are also lower than findings from a South African hospital-based study by Okonta et al. (2014) who finds that $99 \%$ of their sample did not follow any diet (Okonta et al., 2014). With regards to inequality in DDS, our findings show that having a diverse diet is concentrated amongst the better off, as indicated by the positive concentration indices. This finding is also evident from the regression results which show that being within a highest wealth quintile is associated with diverse diets. This finding corroborates the findings from a study by Tiew et al. (2014) who make use of a 
different measure of DDS and finds that in their type 2 diabetes sample, higher income is associated with having a diversified diet (Tiew, Chan, Lye, \& Loke, 2014).

Self-monitoring of blood glucose is an essential component of diabetes self-care and prevention of hypoglycaemia (American Diabetes Association, 2017) as it guides decision making regarding adjustments in medication dosages, exercise regimes and dietary intake. By self-monitoring glucose levels participants become actively involved in achieving targeted glycaemic levels. Using a randomised prospective study of 689 type 2 diabetes patients, Guerci et al. (2003) show that the group who engaged in self-monitoring of diabetes had lower HbA1c levels when compared to the control group (Guerci et al., 2003). The benefits of SMBG in type 1 diabetes has also been demonstrated elsewhere (Diabetes Control and Complications Trial Research Group, 1993). Approximately 92\% of our hospital-based study sample practices self-monitoring of blood glucose levels. This is considerably higher than those reported among type 2 diabetes patients visiting a hospital in Ethiopia in which approximately $84 \%$ did not adhere to self-monitoring of blood glucose (Bonger et al., 2018). Our findings are much higher than an Indian based study that cited unaffordability of glucometers as the reason for poor testing (Mohandas, Bhasin, Upadhyay, \& Madhu, 2018), and also higher than findings from a sample of Chinese Americans in which $27 \%$ monitored their blood glucose daily (Xu, Pan, \& Liu, 2010). These two studies are however not hospitalbased studies. The high levels recorded in our study are perhaps due to the fact that most patients reported having a machine to self-test blood glucose. Consistent with a study by Harris et al. (1993) on a United States population, we found no association between socioeconomic status and self-monitoring of blood glucose amongst people with diabetes within a national health interview survey (Harris, Cowie, \& Howie, 1993).

Non-adherence to diabetes medication is associated with uncontrolled diabetes and an accelerated development of diabetes complications such as retinopathy, nephropathy and neuropathy. Adherence to diabetic medication has been previously investigated (Bagonza, Rutebemberwa, \& Bazeyo, 2015; Kassahun et al., 2016; Krass, Schieback, \& Dhippayom, 2015; Sharma et al., 2014). A systematic review by Krass et al. (2015) reported a wide range of diabetes medication adherence (between $38.5 \%$ and $93.1 \%$ ) which mostly varied as a result of the method used to measure adherence (Krass et al., 2015). Our study shows that $67 \%$ of 
our participants were adherent to diabetes medication. Although our study makes use of the Morisky instrument to measure adherence our finding is consistent with results from a type 2 diabetes hospital-based study in Limpopo, South Africa that used self-reported data recall of taking medication and found $70 \%$ of the participants adhered to treatment (Adegbola et al., 2016). Our results showed that older age was associated with adherence to diabetic medication whilst being non-African compared to being African was associated with none adherence to diabetic medication.

Increased physical activity is associated with reduction in $\mathrm{HbA} 1 \mathrm{c}$ levels (Siddiqui, Bhana, \& Daya, 2018). Our study finds that over $90 \%$ of our sample did not take part in physical activity of at least 2 hours per week. This finding is lower than findings from a sample of Chinese Americans (who lived in Ohio and Chicago) in which $40 \%$ exercised more than five days per week (Xu et al., 2010). Our findings are however consistent with a Mamelodi hospital-based study in South Africa by Okonta et al. (2014) who found that $92 \%$ of the sample did not exercise regularly (Okonta et al., 2014). Our findings are also consistent with findings reported from a study on people with type 2 diabetes living in underserved communities in New York, which found that physical activity was the diabetes self-care behaviour with the lowest rates of adherence (Shaw, Gallant, Riley-Jacome, \& Spokane, 2006). Physical activity was more concentrated amongst those within the higher wealth quintiles. This finding is also supported by findings from the regression which also show that those within higher wealth quintiles were more likely to adhere to physical exercise. Consistent with a hospital-based study in Ghana (Mogre et al., 2017), and a study on Chinese Americans with type 2 diabetes (Xu et al., 2010), we also find that higher education is associated with adherence to exercise.

Our findings show that approximately $15 \%$ of the study participants were smokers. This finding is consistent with findings from a study at Baragwanath Hospital, South Africa which found a self-reported smoking prevalence amongst people with diabetes of $16 \%$ (Gill, Rolfe, MacFarlane, \& Huddle, 1996) and a study from India which found that approximately $14 \%$ of the sample reported smoking in the previous week (Mohandas et al., 2018). Using Cls our study shows that those within lower socio-economic groups were more likely to adhere to non-smoking although we did not find this relation in the regression analysis. Consistent with 
findings from a study in India our study also finds that not smoking is associated with gender, with females more likely to not smoke when compared to males (Mohandas et al., 2018).

Diabetes self-care practices also involve the avoidance of harmful alcohol consumption (Engler, Ramsey, \& Smith, 2013; WHO, 2016a). The IDF recommends a maximum intake of two standard drinks per day (IDF, 2017). Approximately 99\% of the sample abstained from alcohol consumption. This finding is higher than that recorded by a study at public health care facilities in the North West Province, South Africa that found that $65 \%$ of people with diabetes abstained from alcohol consumption (Bhana, Rathod, Selohilwe, Kathree, \& Petersen, 2017). It is possible that patients quit or reduce their alcohol consumption after having been diagnosed with diabetes.

Whilst diabetes self-care is mostly the patient's responsibility (Funnell \& Anderson, 2000), it is well established that health care professionals play a role in supporting diabetes self-care by patients and ultimately improve clinical outcomes. The role of health care professionals in relation to diabetes self-care is gaining an increasing attention in the literature (van Dam, Van der Horst, Van den Borne, Ryckman, \& Crebolder, 2003). To encourage adherence to self-care there is a call for health-care professional and patient interactions to be collaborative rather than directive (Heisler, Bouknight, Hayward, Smith, \& Kerr, 2002). Such interactions encourage patient involvement, via the identification of problems in diabetes management (Beverly, Worley, Prokopakis, \& Ivanov, 2016). The approach promotes shared decision making and allows for the building of a good relationship between patient and health care professional (Heisler et al., 2002) In clinical practice, this will enable health care professionals and patients to make and agree on health care choices together.

\subsection{Limitations}

Our study has some limitations. Diabetes foot care is an integral part of diabetes management. People living with diabetes are encouraged to examine their feet on a regular basis and examine the insides of their shoes before putting them on. Our study however did not collect data on patient foot examination. The hospital-based respondent selection may have missed the inclusion of those people with diabetes who are not seeking care. The degree 
of self-care among that group is not studied. The findings from this study may also be limited by the possibility of social desirability bias during the face-to-face interviews. Furthermore, the use of a cross sectional dataset limits any causal interpretations. The findings from our study are based on data collected from two hospitals in Gauteng and thus may not be applicable to the whole of South Africa. However, we believe that the study provides insights into the self-care behaviours of people with diabetes in patients visiting the two South African public hospitals. Future research should attempt to investigate diabetes self-care management practices of patients attending private health-care facilities in South Africa, in particular qualitative studies that explore the factors influencing diabetes self-care. Furthermore, future research should look into the role of family and friends in the adoption of healthy lifestyle and also focus on the barriers to the adoption of diabetes self-care practices.

\subsection{Conclusion}

This study provided findings on the diabetes self- management practices of patients attending two tertiary hospitals in Gauteng, South Africa and their association with demographic variables. The study showed variation in adherence to diabetes self-care practices amongst the diabetes patients. Whilst high levels of adherence were reported in some self-care behaviours, our findings show that the extent to which patients adhere to the diabetes selfcare behaviours of exercising, dietary diversity and medication are low and might have negative implications for diabetes health outcomes. The Cls showed that dietary diversity and exercise were associated with higher socio-economic status whilst non-smoking was associated with low socio-economic status. A study of this nature is important for health care professionals, in particular health care professionals dealing with economically disadvantaged patients. It is critical for strategies to be developed that help different-socio-economic groups to adopt healthier diabetes self-care practices. Future studies on this topic should also include individuals with diabetes who do not seek care to be able to draw generalized conclusions. 
Chapter 6

Barriers and enablers of diabetes self-care - a qualitative study of people with diabetes in South Africa

Submitted:

Mutyambizi C, Pavlova M, Hongoro C, Groot W. Barriers and enablers of diabetes self-care a qualitative study of diabetic patients in South Africa. 


\section{Abstract}

\section{Background}

Research has shown that diabetes self-care contributes to positive health outcomes. This study explores the challenges people with diabetes face in diabetes self-care and the factors that promote and enable them to adopt recommended diabetes self-care practices. A key objective of the study is to provide evidence for the development of programmes that promote diabetes self-care practices.

\section{Methods}

In this qualitative study interviews were conducted with purposively sampled people with diabetes from 2 public and 1 private diabetes health care clinics in Gauteng, South Africa. Experienced research assistants transcribed and translated the interviews. Data analysis was guided by the Theory of Triadic Influence (TTI) and results presented for the intra-personal, inter-personal and environmental streams.

\section{Results}

Our results show a number of inhibitors and enablers of diabetes self-care that emanate from the intra-personal, inter-personal and environmental streams. Our study identified health related and personal and personality related barriers (intra-personal), non-supportive interactions with health care providers and family and friends (inter-personal) and financial challenges and indirect costs of diabetes as inhibitors of self-care (environmental). Our study shows that the development of self-care expertise and diabetes knowledge (intra-personal), supportive interactions with health care providers and family and friends (inter-personal), employment facilitators and the use of health technology devices are enablers of self-care (environmental).

\section{Conclusion}

Our study demonstrates several factors emanating from 3 steams of influence that act as inhibitors or enablers of diabetes self-care. Effectively enhancing self-care requires multilevel approaches. We recommend programmes that promote self-care be comprehensive and target the intra-personal, inter-personal and environmental streams of influence. 


\subsection{Background}

Diabetes mellitus is a lifelong metabolic disorder characterised by deficient insulin production (type 1) or from a variable combination of inadequate insulin production and inability of the body to effectively use insulin (type 2) (IDF, 2017). Diabetes self-care is an integral part of successful diabetes management to ensure glycaemic control, improved quality of life and prevention of diabetic complications and associated risks of mortality (IDF, 2017; WHO, 2016a). Self-care is defined as the knowledge and skills to perform day to day behavioural routines that control the disease and reduce any adverse impact on health outcomes (Clement, 1995). This involves several recommended behavioural practices such as frequent health checks, healthy eating, adherence to medication and physical activity (Clement, 1995; WHO, 2016a). Compliance with these behavioural practices differs across patients as it is influenced by the various personal and environmental contexts in which self-care takes place. These then act as inhibitors or enablers of self-care.

The inhibitors of diabetes self-care are those factors that deter the individual from undertaking self-care whilst the enablers of diabetes self-care are those factors that promote the individual's ability to undertake recommended self-care practices. Although diabetes selfcare is mostly the patient's individual responsibility (Funnell \& Anderson, 2000), committing to self-care requires support from multiple levels of influence (WHO, 2016a). These include the patients themselves, interactions with family and friends, the physical environment, health care providers and health care system institutions (American Diabetes Association, 2017; WHO, 2016a). Previous studies suggest that health care professionals (Peel, Douglas, \& Lawton, 2007; Williams, Freedman, \& Deci, 1998) social support networks (Baig, Benitez, Quinn, \& Burnet, 2015; Bennich et al., 2017; Osborn, Bains, \& Egede, 2010) and the physical environment (Ong et al., 2018) are crucial to successful diabetes self-care. Therefore, in this chapter, it is presumed that the inhibitors or enablers of self-care originate from the following three streams: a) Intra-personal, i.e. the individual living with diabetes; b) Inter-personal, e.g. friends and family; and c) environmental, e.g. health care systems and policy. Each of these streams provides various resources and levels of support in self-care. 
To the best of our knowledge, in South Africa, qualitative studies on the experiences of persons living with diabetes have either focused on a specific gender (Mendenhall \& Norris, 2015; Van Rooijen, Rheeder, Eales, \& Molatoli, 2002), or on the challenges with glycaemic control (Adeniyi, Yogeswaran, Wright, \& Longo-Mbenza, 2015), dietary guidelines (Ebrahim, De Villiers, \& Ahmed, 2014; Nthangeni et al., 2002) or physical activity (Van Rooijen et al., 2002). Other studies do not exclusively explore the experiences of people with diabetes with self-care but also include hypertensive patients (Kagee, Le Roux, \& Dick, 2007; Murphy, Chuma, Mathews, Steyn, \& Levitt, 2015) or only focus on the challenges associated with selfcare (Booysen \& Schlemmer, 2015; Masupe, Ndayi, Tsolekile, Delobelle, \& Puoane, 2018; Steyl \& Phillips, 2014). Therefore, to contribute to the existing qualitative evidence on diabetes self-care in South Africa, the aim of this study was to gain insight into the multi-level factors that inhibit or enable diabetes self-care at the intra-personal, inter-personal and environmental levels. For the purpose of this study we make use of qualitative data collected from patients attending three diabetes clinics in Tshwane, South Africa. Such evidence is necessary in the design of programmes that support engagement in diabetes self-care.

\subsection{Theoretical framework}

There is a vast number of theories of health-related behaviour in the literature. Whilst sharing common constructs and concepts these theories and the variables included in them have grown over the years, leaving the researcher with dozens of theories to choose from. The TTI originated from a careful review of numerous health-related behaviour theories. The TTI is therefore often described as a comprehensive theory as it combines constructs from many other theories of health-related behaviours (e.g. social learning theory, theory of planned behaviour) by organising these theories and their variables across levels of causation and streams of influence. Therefore, the TTI was used as a theoretical guide for this study.

The TTI is a social cognitive theory, which suggests that variables that influence health can be arranged into two dimensions, (1) levels of causation and (2) streams of influence (Flay, Snyder, \& Petraitis, 2009). The levels of causation are the tiers through which variables influence behaviours and are arranged from causes which have a distant effect (ultimate predictors) to those with a more intermediate effect (distal predictors) to those that are even 
closer to behaviour (proximal or immediate predictors) (Flay et al., 2009). The effect of these tiers of influence flow within what the TTI refers to as three streams of behaviour influence. These are the intra-personal stream, inter-personal social stream and the environmental stream. The intra-personal stream refers to those individual characteristics that influence ones' self-efficacy in conducting certain behaviours. This includes but is not limited to for example personality traits, which for the purposes of our study may be a direct result of the presence of diabetes. The inter-personal social influences are those social levels or microenvironmental influences that work through social normative beliefs regarding behaviour (relations with health care providers, social support). The environmental stream refers to the structural level or sociocultural macro-environmental factors that influence attitudes about certain behaviours and interactions with broader institutions (e.g. hours spent at health facility, health care system factors impeding diabetes management) (Flay et al., 2009; Snyder \& Flay, 2012).

The TTI has been applied in many behavioural domains such as chronic diseases (Earnshaw et al., 2018), drinking and driving (Greene, Murphy, \& Rossheim, 2018), sexual violence (Petersen, Bhana, \& McKay, 2005) dietary behaviours and substance abuse (Snyder \& Flay, 2012). In South Africa, research was yet to apply the analytical lens of the TTI in assessing diabetes related behaviour. The analysis of our data are therefore based on the three streams of the TTI by assessing how individual factors, inter-personal and environmental factors either inhibit of enable self-care in people with diabetes.

\subsection{Methods}

\subsubsection{Study design}

This study was conducted as part of a large study on the costs and management of diabetes amongst people with diabetes. More specifically, it was conducted to complement the findings from Chapter 5. Qualitative methods using semi-structured interviews were used to address the study objectives. Given our research aims qualitative research allows us to explore and understand meanings and experiences. The research method allows us to obtain 
a rich and comprehensive understanding of patient-oriented inhibitors and enablers of diabetes self-care.

\subsubsection{Assessment tool}

To ensure that key topics related to the study objectives were covered during the interview the interviewers used an interview guide with open ended questions (see Appendix $H$ ). The interview guide was developed after an extensive literature search and based on past literature. The guide allowed patients the freedom to express their feelings whilst remaining true to their story during the interview process. Interview questions were related to patient experiences with diabetes self-care. To elicit rich and detailed answers from participants general probing was used (What is your opinion? Can you explain further? etc.). As recommended in the literature, pre-test was conducted prior to the commencement of the interviews (Elo et al., 2014). Data from this pre-test are not added to this final analysis.

\subsubsection{Data collection}

Data for this chapter were collected at the same hospitals as the quantitative data used in Chapter 3 and Chapter 4 . As the researcher recruited participants from health facilities, a purposive sampling approach was adopted to recruit patients based on their age (above the age of 21 years), taking diabetic medication and visiting the clinics during the data collection periods. Purposive sampling enabled us to target the individuals from whom we could get the best possible information on the research topic. Written informed consent was obtained from all participants (see Appendix G).

For the data collection in the public hospitals four research assistants were recruited and trained to conduct the data collection. Participants were approached by the research assistants whilst they were waiting for consultation with the medical doctor and were invited to participate in the study. If they consented, participants were then interviewed at the clinic before or after their consultation.

For the data collected in the private clinic, the principal researcher conducted all interviews. Prior to the data collection, the medical doctor at the clinic was provided with detailed 
information regarding the study and provided consent for the investigator to speak to consenting patients. During consultation, the medical doctor informed each patient about the study and directed patients who agreed to take part in the study, to a private room in the clinic in which the principal investigator then conducted all interviews.

To overcome language barriers and ensure participants were comfortable and confident with their responses, the interviews were conducted in the respondents preferred language. Prior to the qualitative interview, a structured questionnaire was used to collect demographic information from the participants. The interview began with general information in which the interviewee introduced themselves and the study. All interviews were audiotaped. To ensure comprehension and completeness data collection continued until interviews did not produce any new information.

The quality of the research process was assessed using the Consolidated Criteria for Reporting Qualitative Research (COREQ) checklist (see Appendix I).

\subsubsection{Data analysis}

The information collected from patients was transcribed. Interviews that were not conducted in English, were translated prior to data analysis. To ensure the accuracy of transcriptions, several transcriptions, were randomly selected and checked for accuracy. Transcribed interviews were then entered into Atlas.ti, a computer program that is used in the analysis of textual data by allowing the researcher to arrange, reassemble and manage text in order to extract meaning. Data analysis was conducted using content analysis and analysis was guided by Elo and Kyngas (Elo \& Kyngäs, 2008). First, all transcripts entered into the software, were read several times. A set of preliminary descriptive codes was developed based on the transcripts review and domains of the TTI (e.g lack of self-control, cost of diabetic diet and relationship with health care providers). Next, each transcript was coded using this set of established codes. During this process, codes were refined and new ones had to be created. An analysis of the relationships between codes was then conducted and then organised to the core themes as guided by the TTI. Comments outside the scope of this study were 
excluded from the analysis. Our results section further elaborates on the identified themes and excerpts from the transcripts selected to support the study main findings.

\section{$6.4 \quad$ Results}

\subsubsection{Demographic characteristics}

Twenty eight interviews were conducted by the principal researcher and the four data collectors. Whilst 18 patients were from public sector clinics, 10 participants were from the private sector diabetes clinic. The sample interviewed was diverse in terms of demographic data. Participants' age ranged from 21 years to 80 years. One participant declined disclosing their age. Of the 28 patients interviewed, the majority were within the age group of 36 to 60 years (52\%). All participants were taking diabetic medication, $54 \%$ were female, $46 \%$ were male. Of the 28 participants interviewed $46 \%$ were African, $54 \%$ were non-African (Coloured, Indian or White), $54 \%$ were married and $64 \%$ had a family history of diabetes. Most participants reported that they had a tertiary education (50\%). Length of time since diabetes diagnosis averaged 15 years (median 12 years). Twenty one participants reported the presence of other illness such as high blood pressure and kidney disease.

\subsubsection{Themes}

Table 6-1 provides a summary of the results. A thorough explanation of these findings is provided below. The findings are presented in accordance with the streams theorised to influence diabetes health self-care behaviour in the TTI; intra-personal stream, inter-personal social stream and the environmental stream (Flay et al., 2009). Participants' range of experiences regarding diabetes self-care can be broadly organised into these streams.

\subsubsection{Theme 1: Intra-personal stream}

This theme relates to the intra-personal factors that are linked to personality traits that are either barriers or enablers of diabetes self-care. Intra-personal level factors were commonly referred to as barriers to self-care. We categorised these into (1) personal barriers and personality traits and ( 2 ) health related barriers. Personal and personality barriers of feeling 
lazy, forgetfulness, being tired, being too busy, lack of self-control and a failure to overcome childhood habits were frequently described as barriers to self-care by participants in the study. Forgetfulness was often cited as a reason for not adhering to diabetic medication whilst being lazy, tired and busy were often cited as barriers to engaging in physical activity. Many participants reported a lack of self-control by not being able to overcome cravings for food that they should not be eating. These intra-personal barriers are emphasised in the following quotes from some of the participants:

It is difficult to get used to not using sugar, oil and fats. I know there is a difference, I feel the difference when I am eating right but it is just the cravings, you get the cravings for those oily food and I can't resist, that is the only problem. [Participant 23, 46 years old, female]

It would be nice if you been brought up in a way where you do exercise, but once you get older and you have been working, you don't always do the exercise as you should. [Participant 17, 50 years old, female]

I am lazy, I am lazy to exercise. That I know, but I have to push myself, body changed, it is not the same as it was before the illness. It was energetic before. [Participant 1, 32 years old, male]

Well I sometimes fall asleep while watching TV then when I go up I test my sugar but I always forget if I injected or not so I didn't know what to do and you can't inject 2 times so l'd just leave it. [Participant 12, 22 years old, female]

The health-related barriers to self-care were also commonly reported by participants. Participants felt that depression was a barrier to self-care, the presence of diabetes complications, comorbidities or multi-morbidity meant that engaging in physical activity was painful. Therefore, they were unable to adhere to physical activity recommendations. Four respondents reported that the development of medication side effects led to them not taking their medication or adjusting their own medication dosage.

I eat my vegetables but fried. It is not healthy and I know that but to get out of that it is difficult. It is a challenge to get out of that. You get depressed and you feel like it is 
just another day, another diabetes day. Inject, eat, inject, eat. You just carry on without that positive challenge of that you going to get better, I am going to get healed, there is no healing in this situation. ... I ignored the help and I got stuck and depressed and now it feels like it's too late because it has done enough damage. [Participant 23, 46 years old, female]

Unfortunately, I'm not able to do any exercises, even when I walk when I go to Pick \& Pay its difficult for me because I get chronic pain all the time. [Participant 11, 80 years old, male]

So at the moment you can't feel anything under my feet and unfortunately that also affecting my balance, I use to ride with my bicycle but I can't anymore because I don't have a feel on the pedals you know, so very afraid to fall. [Participant 15, 76 years old, male]

Sometimes I have fallen after drinking these tablets I collapsed then I didn't drink them again I bring them back I say these make me sick I collapsed they [doctors] say ok but the following time I find them there [in the prescription]. ... That is why a lot of people are throwing tablets away the other will take it and put it in the dust bin which is not good because some people still need them so I have told them, they laughed at me.... Its either I'm giving to these people in that clinic or I'm throwing it away, let them listen to us we will be pleased because some of these things we are not drinking because it's causing problems. [Participant 8, 69 years old, female]

Although intra-personal level factors were largely characterised as a source of barriers to diabetes self-care, some participants highlighted some ways in which individual level factors acted as enablers of self-care. Participants mainly focused on (1) diabetes knowledge and (2) development of self-care expertise as a diabetes self-care enabler. Some patients reported that the diabetes knowledge they had, enabled them to make decisions regarding self-care practices. Development of self-care expertise was influenced by the time since diagnosis and the presentation of diabetic complications which acted as turning points for diabetes selfcare. People with diabetes who felt they had been diagnosed with the chronic illness long ago, were confident with their diabetes self-care due to developing an awareness of their 
bodies and an understanding of what sort of behaviour works best for them. Four participants described turning points in their lives as a result of the development of complications or hospitalisation that led to a strong commitment to diabetes self-care. These intra-personal enablers are emphasised in the following quotes from some of the participants.

I am able to manage it because, I was told not to eat certain food. For example, salt and sugar. I don't use them a lot. I measure them. If I feel that whatever am eating has a lot of salt, I don't eat it. I eat appropriate things. [Participant 3, 77 years old, female] But as time has gone by you get to understand it more and you actually even know how you feel and so on. So you do understand it more and you can even just tell on your own, you can feel the symptoms and it's actually manageable and you know how to treat it, so it improves as you go along with time. [Participant 27, 21 years old, female]

At the moment am used to it. I am no longer craving them because it's been a long time since I changed my eating habits. I used to love cakes and cool drinks, but not anymore. [Participant 3, 77 years old, female]

In the past there were complications with me, due to not adhering to those recommendations. Nonetheless, I had to change my lifestyle. Adapted a new eating habits and diet, I had to change my mind-set and strictly follow those recommendations because I do not think that I would still be alive, had I not followed those recommendations. [Participant 1, 32 years old, male]

\subsubsection{Theme 2: Inter-personal social stream}

In our study inter-personal level influences of diabetes self-care are those that came from interactions and relationships with family, friends and health care providers. This stream reflects an exchange of resources between two or more people. Participants reported back on inter-personal level factors that acted as barriers to self-care. Non-supportive interactions with family and friends through their non-diabetic lifestyle influences and a lack of family support acted as a barrier to diabetes self-care. This barrier was common in both private 
sector and public sector patients. Many participants referred to how hard it is to resist unhealthy food during family gatherings.

I am trying, even when I eat something that is bad for my health, it is there at the back of my mind that I know I am not expected to eat this thing. ... Sometimes it's because you are craving, especially when you are with other people. [Participant 6, 35 years old, male]

I work for a man so have a roof over my head I have food, they do not have to make particular diet for me because am diabetic l eat what they eat. [Participant 22, 54 years old, male]

It 's difficult to cook separately for them ... I have to cook like am cooking one meal and we all eat together that's the problem because am not allowed to eat what they are eating. [Participant 20, 39 years old, female] 
Table 6-1 Coding frame

\begin{tabular}{|c|c|c|c|}
\hline TTI Stream & Global theme & Organizing theme & Basic theme identified in transcript \\
\hline \multirow{12}{*}{ Intra-personal } & \multirow{9}{*}{$\begin{array}{c}\text { Individual factors as a } \\
\text { diabetes management } \\
\text { inhibitor }\end{array}$} & \multirow{3}{*}{ Health related barriers } & Diabetes complications, comorbidity or multimorbidity \\
\hline & & & Side effects of medication \\
\hline & & & Depression \\
\hline & & \multirow{6}{*}{$\begin{array}{l}\text { Personal and personality } \\
\text { barriers }\end{array}$} & Being lazy \\
\hline & & & Being forgetful \\
\hline & & & Being tired \\
\hline & & & Being too busy \\
\hline & & & Lack of self-control \\
\hline & & & Childhood habits \\
\hline & \multirow{3}{*}{$\begin{array}{c}\text { Individual factors as a } \\
\text { diabetes management } \\
\text { enabler }\end{array}$} & \multirow{2}{*}{$\begin{array}{l}\text { Development of self-care } \\
\text { expertise }\end{array}$} & Individual turning points due to illness presentation \\
\hline & & & Time since diagnosis \\
\hline & & Diabetes knowledge & Awareness regarding self-care practices \\
\hline \multirow{5}{*}{$\begin{array}{l}\text { Interpersonal or } \\
\text { social }\end{array}$} & \multirow{4}{*}{$\begin{array}{c}\text { Interactions and } \\
\text { relationships as a diabetes } \\
\text { management inhibitor }\end{array}$} & \multirow{2}{*}{$\begin{array}{l}\text { Non-supportive interactions } \\
\text { with family and friends }\end{array}$} & Non-diabetic lifestyle influences \\
\hline & & & Lack of family support \\
\hline & & \multirow{2}{*}{$\begin{array}{l}\text { Non-supportive interactions } \\
\text { with health care providers }\end{array}$} & Information from health care providers not enough \\
\hline & & & Nurse attitudes \\
\hline & & & Reminders, material and care assistance from friends \\
\hline
\end{tabular}




\begin{tabular}{|c|c|c|c|}
\hline TTI Stream & Global theme & Organizing theme & Basic theme identified in transcript \\
\hline & & & $\begin{array}{l}\text { Financial support, reminders, material and care assistance } \\
\text { from family }\end{array}$ \\
\hline & $\begin{array}{l}\text { Interactions and } \\
\text { relationships as a diabetes }\end{array}$ & family and friends & $\begin{array}{l}\text { Family history and knowledge of diabetes reinforced self- } \\
\text { care }\end{array}$ \\
\hline & management enabler & & Exercise groups \\
\hline & & Supportive interactions with & Good patient doctor relationship \\
\hline & & health care providers & Diabetes information from doctors \\
\hline \multirow{9}{*}{ Environmental } & \multirow{6}{*}{$\begin{array}{l}\text { Physical environment and } \\
\text { institutions as a diabetes } \\
\text { management inhibitor }\end{array}$} & \multirow{3}{*}{ Financial challenges } & Health care costs \\
\hline & & & Cost of a diabetic diet \\
\hline & & & Medication and glucose monitoring costs \\
\hline & & \multirow{2}{*}{ Indirect costs of diabetes } & Time spent seeking care \\
\hline & & & Loss of wages and job \\
\hline & & Employment barriers & Work related commitments \\
\hline & \multirow{3}{*}{$\begin{array}{c}\text { Physical environment and } \\
\text { institutions as a diabetes } \\
\text { management enabler }\end{array}$} & \multirow{2}{*}{ Employment facilitators } & Material support from employer and work environment \\
\hline & & & Work-related physical activity \\
\hline & & Use of technology devices & Use of technology devices \\
\hline
\end{tabular}


Patients visiting public hospitals reported on non-supportive interactions with health care providers as a barrier to self-care. Four participants thought that during their interactions with medical doctors they were not given enough information regarding diabetes and diabetes self-care. One of the patients attributed this to the short consultation times whilst others thought this was due to seeing a different medical doctor each time they came for consultation. Three patients visiting the public health facilities also thought that nurses did not treat them well. Nurses were accused of speaking to patients in an irritable way or simply ignoring patients when they needed care. These non-supportive interactions are emphasised in the following quotes from some of the participants.

The speed with which they have to push patients through doesn't allow them to really deal with the patient in a right way. [Participant 21, 75 years old, male]

You get a different doctor and instead of having one doctor that follows you through, you going to ten million doctors just explaining the whole thing all the time and lots of the things all the time get missed on the way... On the doctors' side you are too scared to ask them because you do not know them that much, you haven't built up a relationship like your general practitioner, so you go in and give half a story, you don't fulfil everything that you want to say. [Participant 19, 57 years old, female]

Sometimes the thing of a different doctor treating you every now and then gets in the way because they will each give you different opinion and keep changing you and your levels are not monitored due to lack of information. [Participant 27, 21 years old, female]

Sometime back I experience one diabetic woman falling there waiting for the medicine, which is very very bad she could die there because we are all patients there sitting no nurse, no help no person to help there their all gone more especially if it's their time for tea time lunch time they will just leave you there and go they don't care, they don't care at all these nurses here I don't know how can I put it sorry to say that but they don't care. [Participant 5, 44 years old, male]

Participants visiting both the public and private sector clinics frequently reported back on inter-personal level factors that acted as enablers of diabetes self-care. Supportive 
interactions with family and friends through the provision of reminders, material and care assistance from friends and family, the provision of financial assistance from family, being part of exercise groups and interactions with family or friends with diabetes knowledge or a history of diabetes provided reinforcement for diabetes self-care practices amongst patients.

There (is) a lady that I work with, we are very close, sometimes when we chatting, she will be like have you taken your medication, have you eaten you see. When I am with them, they know I am not supposed to eat certain things. Even when l eat sugary staff, they usually remind me that hey not too much. [Participant 6, 35 years old, male]

I exercise once a week. Every Tuesday we meet with other grannies to exercise ... we exercise, or if we can't we walk, sometimes we do aerobics. [Participant 3, 77 years old, female]

The support that I get from my family helped a lot and also my paternal grandmother was very instrumental in helping me accept. She had diabetes as well ... She explained everything to me about diabetes. [Participant 4, 36 years old, female]

I try to think about the good things and look forward to getting to the hospital to get my medication so I can see other people who are also diabetic. It motivates me to do more and be positive about it. [Participant 23, 46 years old, female]

Furthermore, supportive interactions with health care providers in the form of good-patient doctor relationships and diabetes information from medical doctors came out as a facilitator for diabetes self-care practices.

If I need anything, I can call even the doctor's number, one week my sugar was up so doctor gave me her number and she SMSed me at 10 o'clock in the night. [Participant 12, 22 years old, female]

\subsubsection{Theme 3: Environmental stream}

The most frequently reported environmental level barriers to self-care in our study was due to financial challenges as a result of the high diet costs, the costs of medication and glucose 
monitoring strips and health seeking costs. These barriers were common amongst both private and public sector respondents.

The problem with diabetes is that the food is very expensive, the food that you eat is not normally the kind of food that you get anywhere like spaza shops and all that, because even bread you need to go the mall to get special bread for diabetes, you have to eat low-fat milk. [Participant 2, 35 years old, male]

Yoh! It is draining financially because you end up eating wrong foods because you reach a point whereby you no longer able to afford the required food. [Participant 25, 46 years old, female]

I have to must test myself often and I use a lot of sticks and that stresses me as well because I can't afford to buy sticks all the time. The hospital can't give me more, enough or what I need to test myself. [Participant 23, 46 years old, female]

Other indirect diabetes costs such as time spent seeking care and loss of wages and employment due to diabetes were reported by patients visiting the public clinics.

Yoh! They slow, we start with the "file "section, to the Pharmacy, waiting for the doctor as well as during consultation, they all very slow. We end up staying at the hospital for hours and becomes hungry. Considering that we diabetic people we need to constantly eat. [Participant 25, 42 years old, female]

Diabetes has affected me a lot, at this stage I lost my job because of diabetes, I was working hard labour, another time I found that my sugar is very low and fell while at work then they sent me home and then when I go back they told me that we don't need you anymore. [Participant 2, 35 years old, male]

A few public sector patients also reported that employment barriers due to work related commitments often resulted in non-adherence to diabetes self-care practices.

It's very difficult especially when working, to carry stuff around, I mean like in the heat outside its very hot and caring insulin with you, you have to make sure that it 
refrigerated and everything. Sometimes when you are taking out clients, you can't take it with you, so it's a little bit difficult... With being out on the road and everything like that, seeing clients, you just don't get that time to actually just say excuse me to the client, I got to go and inject and something like that, so no it doesn't get done all the time at the right time. [Participant 19, 57 years old, female]

Although the environmental stream was largely characterised as a barrier to self-care, three participants noted employment as enablers of diabetes self-care. Participants reported employment enablers such as work-related physical activity and material support from employer.

I can walk the whole day. With exercise I don't have a problem ... where I am working is also is very active, so you have to do up and down the whole day. [Participant 24, 37 years old, male]

When I am at work, I have no problem in following the diet recommendation because my employers maintain a healthy lifestyle and their grocery consists of vegetables, fruits and all the items required. [Participant 25, 42 years old male]

Some participants reported that the use of health technology devices such as an insulin pump and glucose monitors, helped with adhering to diabetic medication. Whilst the use of the insulin pump was restricted to private sector patients the use of glucose monitoring machines, was also reported by public sector patients.

You don't forget to take your insulin, the other thing is also you, you actually test your blood glucose more often, I think sometimes people forget or they get lazy, whereas with insulin pump you need to feed information into the machine cause the pump has got a target range that it must keep your blood glucose at. [Participant 14, 48 years old, male] 
I'm just sorry that I did not start earlier with the pump it's more scientific its better, you control yourself much better with the pump than with the injections and that type of thing. [Participant 15, 76 years old, female]

I listen to the radio and every time I hear them reading the news, so it reminds me now you must do this, you must test yourself and every hour I must report ... I take note every day, I write down what I eat, what I do and when I test myself and then I evaluate myself according to those [glucose readings]. [Participant 23, 46 years old, female]

\subsection{Discussion}

Past research in South Africa has documented the high prevalence of treated but uncontrolled diabetes (Govender, Gathiram, \& Panajatovic, 2017; Pinchevsky, Butkow, Chirwa, \& Raal, 2017; Stokes et al., 2017), which is reportedly an outcome of poor self-care practices (American Diabetes Association, 2017). This qualitative study sought to assess the ways in which intra-personal, inter-personal and environmental factors act as inhibitors or enablers of such self-care. The TTI was used to draw attention to the intra-personal, inter-personal and environment factors that influence diabetes self-care. Our study highlights how the diabetes self-care decision by individuals cannot be separated from the larger context of social relations and the environment in which people with diabetes must live in. Our findings show that the three streams present factors that promote or inhibit diabetes self-care. Recognising these factors is essential to development of programmes that assist with enhancing self-care.

Inhibitors and enablers of diabetes self-care at the individual level were the most commonly reported by all patients. Our results indicate that personal and personality factors are a major barrier to diabetes self-care. Through the individual stream the study shows that the level of self-care may depend on the severity and limitations imposed by diabetes itself. Responses of feeling tired and lazy as inhibitors of self-care may be a direct result of having uncontrolled diabetes. Our finding that various personal barriers, such as laziness or lack of time and selfcontrol, influence self-care, which is consistent with findings from South African and international studies (Chlebowy, Hood, \& LaJoie, 2010; Steyl \& Phillips, 2014). Furthermore, as diabetes may affect other body organs and may result in complications, it is not surprising 
that like many other studies (Steyl \& Phillips, 2014), the participants in our study expressed that health-related factors such as diabetes complications, resulted in ineptitude in self-care. At the same time, we described the ways in which intra-personal factors acted as enablers of self-care. The development of expertise in diabetes self-care has been well documented and is characterised by the development of increasingly refined skills, knowledge and abilities to effect changes in one's response to diabetes management and develop control over their disease management (Paterson \& Thorne, 2000). Consistent with previous research, we find that some patients felt that they had developed expertise in their disease management, through developing an awareness of their bodies and an understanding of what sort of behaviour works best for them (Adu, Malabu, Malau-Aduli, \& Malau-Aduli, 2019).

At the inter-personal and environmental streams some differences were evident in the responses from patients who visited public sector clinics versus those who visited the private sector clinic. From a structural perspective, in South Africa health care is provided by private and public health facilities separated by socio-economic lines (National Department of Health, 2011a). The public sector, which is reported to serve the majority of the population, is poorly resourced and funded through the fiscus and OOP payments. The private sector on the other hand is staffed by approximately $70 \%$ of the country's medical doctors and financed mostly by health insurance and OOP payment (Mayosi \& Benatar, 2014; National Department of Health, 2011a). The private sector commonly serves the formally employed and wealthier population (Ranchod et al., 2017). The inequalities that exist between these two sectors was evident in some of the inter-personal and environmental stream expressions by the patients visiting the diabetes clinics. We present a discussion of the key findings within each stream below.

At the inter-personal level, our results support findings from empirical work that shows that social interactions and relations with family, friends and health care providers can act as an inhibitor or enabler of diabetes self-care (Miller \& DiMatteo, 2013). Consistent with other studies, participants expressed that they found it particularly difficult to adhere to healthy lifestyles when other family members were not (Booysen \& Schlemmer, 2015). In particular, they reported that sticking to a healthy diet was difficult if other family members are eating unhealthy food. Participation in social gatherings was reported as a huge impediment to self- 
care. Similar to previous studies, we find that patients in both the public and the private clinics experienced various types of social support (e.g. material, emotional or financial) that promoted self-care (Chlebowy et al., 2010; Miller \& DiMatteo, 2013). In this study patients who had a family of history of diabetes reported having an understanding of the disease and how best to manage it.

Interactions with health care providers was both an enabler and inhibitor of diabetes selfcare. Additionally, patients in our study felt that the information provided to them by health care providers was not enough to help with self-care. This finding was salient in patients making use of the public health care system and could point to problems related to limited patient-doctor consultation times in public hospitals as previously reported. At the same time, even though social interactions and relations seemed to act as a barrier to self-care, they can also act as an enabler. A good patient-doctor relationship and communication is an essential element of care (Ha \& Longnecker, 2010). In particular, for people living with diabetes, consultations should not focus on laboratory test results and diabetes guidelines but should incorporate a focus on understanding the patient's diabetes experiences and treatment goals (Freeman \& Loewe, 2000). In their interactions with health care providers, public sector patients felt the nurse had attitudes and did not treat them well. This feature may be a result of how overburdened and under-resourced public health care systems are. On the other hand, some public sector patients thought they had a good patient-doctor relationship that fostered diabetes self-care.

In line with previous research our study highlights the importance of context when assessing health related behaviours (Greene et al., 2018). Our results show that environmental or structural level factors are also important in determining self-care. For the public sector patient's time spent at health facility was a commonly reported issue. Our finding is similar to previous studies in which patients report how hospital waiting times inhibits diabetes selfcare (Booysen \& Schlemmer, 2015; Steyl \& Phillips, 2014). Consistent with previous studies in South African (Adeniyi et al., 2015; Booysen \& Schlemmer, 2015; Steyl \& Phillips, 2014) and international studies (Adu et al., 2019), financial challenges were reported to be a huge inhibitor of self-care. Although more frequently reported by patients in the public diabetes clinics, some participants in the private sector also felt diet cost was a huge barrier to 
compliance with dietary recommendations, often due to the lack of money. Similar findings are evident in earlier studies done on the challenges with diabetes management in South Africa (Booysen \& Schlemmer, 2015; Steyl \& Phillips, 2014). Furthermore, patients found it difficult to maintain self-care in jobs that required a lot of travelling. Similar findings were reported in other studies too (Adu et al., 2019). The environmental stream also acted as an enabler of diabetes self-care through various employment facilitators and the use of healthrelated technologies. The benefits of using health technologies devices in diabetes self-care has also been previously reported in other studies (Adu et al., 2019). Although reported as costly and unaffordable, access to health insurance for private sector patients, meant that private sector patients had access to insulin pumps that patients in the public sector do not have.

\subsection{Limitations}

Our study is subject to some limitations. Our study is limited to people who access formal health care services and is restricted to three diabetes clinics in a single South African province. The expressions on diabetes self-care may be influenced by the psychosocial environment and may not be applicable to South Africa in general. The absence of explicit queries or references to culture in our study may be another limitation of the study. Our study also did not explore the role of culture beliefs, attitudes and practices in diabetes self-care. Future research should pay special attention to the broader cultural context within the environmental stream, which may influence self-care. Despite these limitations the study does provide useful insights into understanding the different ways in which intra-personal, inter-personal and environmental factors act as inhibitors or enablers of diabetes self-care.

\subsection{Conclusion}

This chapter highlights a number of inhibitors and enablers of diabetes self-care among the study participants, emanating from various streams of influence. Findings from the study suggest the need for interventions at the intra-personal, inter-personal and the environmental streams. These interventions may take the form of improving diabetes education (intra-personal level), improving awareness of diabetes and recommended management practices by family members of the individual living with diabetes (inter- 
personal level) and health care access financial protection for people with diabetes (environmental level). The latter is particularly important for the achievement of universal health coverage in South Africa. Addressing the inhibitors of diabetes self-care will improve quality of life and prevent the development of diabetic complications and associated risks of mortality. 
Chapter 7

The extent and determinants of diabetes and cardiovascular disease comorbidity in South Africa - results from the South African National Health and Nutrition Examination Survey

(SANHANES-1)

This chapter draws upon:

Mutyambizi C, Chola L, Groot W, Pavlova M, Labadarios D, Hongoro C. The extent and determinants of diabetes and cardiovascular disease comorbidity in South Africa-results from the South African National Health and Nutrition Examination Survey (SANHANES-1). BMC Public Health. 2017;17(1):745. 


\section{Abstract}

\section{Background}

Diabetes is a major health problem and cause of death worldwide. It is predicted that the prevalence of diabetes will increase from 415 million in 2015 to 642 million in 2040. However, the burden of diabetes in LMICs is not clearly understood, particularly its interaction with other chronic illnesses. This study investigates the self-reported prevalence of and factors associated with diabetes and cardiovascular comorbidity in South Africa.

\section{Methods}

Data used in this study are from the 2012 SANHANES-1. Diabetes and CVD comorbidity was defined as the coexistence of diabetes plus one or more CVDs reported at the time of the survey. This study makes use of multinomial logistic regression models to analyse the relationship between diabetes - CVD comorbidity and several predictors including race, income, socio-economic status and obesity.

\section{Results}

According to the survey data we analysed, $5 \%$ of South Africans aged 15 and above had selfreported diabetes in 2011-2012. Among those with self-reported diabetes, $73 \%$ had at least one additional cardiovascular chronic illness. Diabetes and its CVD comorbidity were more prevalent in Africans (66\%), females (66\%), those who lived in urban areas (75\%), had secondary education (44\%) and were unemployed (62\%). Factors strongly associated with diabetes - CVD comorbidity were older age (Odds ratio [OR] 1.09; 95\% Confidence Interval 1.06-1.12), high household income (0.27; 0.10-0.76) versus low-income, moderate (0.33; 0.11-0.96) and good self-rated health $(0.24 ; 0.08-0.68)$ versus bad self-rated health, occasional (0.29; 0.10-0.88) and regular smokers $(0.25 ; 0.12-0.53)$ versus non-smokers and physical activity $(0.15 ; 0.03-0.68)$ versus no physical activity.

\section{Conclusion}

The study provides useful insight into the factors associated with CVD comorbidity in people with diabetes. The findings indicate that there are differences in the factors associated with diabetes and those associated with diabetes - CVD comorbidity. The study provides 
information, which can be used to design programmes that encourage healthy lifestyles in people living with diabetes. 


\subsection{Background}

Although the prevalence of both type 1 and type 2 diabetes is increasing all over the world, type 2 diabetes is reported to be the most common type of diabetes globally (IDF, 2015). Diabetes and its related illnesses is an increasing cause of premature death and it is estimated to have caused significantly more deaths in 2015 than in 2005. Mortality from diabetes increased by $32.1 \%$ in the period 2005 to 2015 ( 1.5 million deaths globally in 2015) whilst mortality from diabetic kidney disease increased by $6.4 \%$ in the same period ( 418000 deaths globally in 2015) (GBD 2015 Mortality and Causes of Death Collaborators, 2016). In South Africa, diabetes ranked third amongst the top ten causes of death in 2014 (Statistics South Africa, 2015). The statistics of the rising prevalence and mortality due to diabetes globally makes it one of the biggest public health concerns of the $21^{\text {st }}$ century (IDF, 2015).

As mentioned in Chapter 1, along with an increased prevalence of diabetes as a single cause of morbidity, there is increasing evidence that individuals with diabetes experience one or more micro- or macro-vascular complications, including but not limited to CVDs, blindness, peripheral neuropathy and kidney disease (Deshpande, Harris-Hayes, \& Schootman, 2008). Individuals with diabetes are at an increased risk of CVDs such as stroke, heart attack and heart failure when compared to individuals without diabetes (IDF, 2001) and the presence of high blood pressure contributes to increased risks of CVD complications (IDF, 2015). In addition, heart disease and stroke account for the highest mortality rates in people with diabetes when compared to those without (Deshpande et al., 2008). In Africa, delayed diagnosis often leads to the development of diabetic complications (Motala \& Ramaiya, 2010). Heart disease is estimated to affect $20 \%$ of people with diabetes whilst approximately $50 \%$ of people with diabetes suffer from heart muscle diseases (Motala \& Ramaiya, 2010). It is also estimated that renal failure is the leading cause of death amongst hospitalised people with diabetes and that diabetes is the leading cause of amputations in Africa (Motala \& Ramaiya, 2010). This suggests that the analysis of diabetes comorbidity is a relevant research topic in African countries, including South Africa.

Comorbidity has various definitions (Jakovljevic \& Ostojic, 2013) but it is usually referred to as the presence of one or more additional illnesses in an individual affected by the index 
disease under study (Nagl, Witte, Hodek, \& Greiner, 2012). The concept of comorbidities and complications has often been confused and used interchangeably in epidemiologic research (Ording \& Sørensen, 2013). Whilst complications occur after diagnosis of an index disease and are a consequence of the index disease, comorbidities can be diagnosed before or after diagnosis of the index disease and are not a consequence of the index disease (Ording \& Sørensen, 2013). In some cases, separating the two may be a complex task when a condition does not clearly fit the criteria of being a comorbidity or complication (Ording \& Sørensen, 2013). For example, high blood pressure is a common complication of diabetes but may also occur in isolation. Therefore, this study makes use of a very broad definition of comorbidity. In this study comorbidity refers to a situation where an individual who has diabetes, also has one or more CVDs such as heart disease, stroke and high blood pressure. Comorbid chronic conditions, such as CVDs, can increase the complexity of the disease burden in people with diabetes, directly worsening their health outcomes whilst also placing a heavy burden on health care systems (IDF, 2001).

This is of great concern in South Africa given the rise in mortality due to NCDs within the country (National Department of Health, 2013). Due to the anticipated increases in the prevalence of diabetes, research into its interactions with other conditions, such as CVDs, is becoming increasingly important. The occurrence and burden of two or more illnesses in one person has mostly been studied in high income countries whilst research in LMICs is relatively scarce (Khanam et al., 2011). There is limited evidence of chronic disease comorbidities in South Africa in the published literature, and to our knowledge, there is no study on diabetesCVD comorbidity. Alaba and Chola (2013) showed that the prevalence of chronic disease multi-morbidity in South African adults was $4 \%$ of the adult population, with obesity, smoking and depression being among the major determinants (Alaba \& Chola, 2013). However, their study did not specifically focus on people with diabetes. In our study, we highlight the CVD comorbid conditions common among people with diabetes and examine the association of diabetic comorbidity with various other determinants of health. This is important given the increasing prevalence of diabetic complications and potential implications on the health care system. An understanding of the factors associated with diabetes and its comorbid conditions will therefore help influence decision making and interventions targeted at preventing and 
managing diabetic comorbidity. This paper investigates the self-reported prevalence of and factors associated with diabetes and cardiovascular comorbidity in South Africa.

\subsection{Methods}

\subsubsection{Data}

Data were taken from the 2012 SANHANES-1 which was used and described in Chapter 4.

\subsubsection{Measures}

In the SANHANES-1 survey, the adult questionnaire provided information on diabetes and various other CVD conditions. Respondents were asked to state whether or not a physician or nurse or health worker at a clinic or hospital had told the respondents that they have or have had diabetes or any of the following CVD conditions; high blood pressure, stroke and heart disease. As such the diabetic comorbidities considered in this study are heart disease, high blood pressure and stroke. The prevalence of these illnesses was estimated using selfreported previously diagnosed health conditions. The SANHANES questionnaire did not ask respondents to specify the type of diabetes they had. In the analysis, these health conditions were used to create a categorical dependent variable of comorbidity ranging from 0 to 2 . The categorical variable took on the following values; $0=$ respondent did not have diabetes or any CVD, 1 = presence of diabetes without CVD comorbidity, $2=$ presence of diabetes with CVD comorbidity (i.e. presence of diabetes and one or more of the chronic diseases mentioned above at the time of the survey).

The selection of independent variables was guided by the Commission on Social Determinants of Health (CSDH) model (Solar \& Irwin, 2007). The model distinguishes structural and intermediary determinants of health. The structural determinants refer to the mechanisms that generate stratification in the society which in turn influences or defines individual social economic position (Solar \& Irwin, 2007). The CSDH framework postulates that these structural determinants configure health opportunities based on economic status. Structural determinants include factors such as education, income, socio-economic position, and other social stratifiers, such as gender, ethnicity and age. The intermediary determinants include 
behavioural factors, psychosocial factors, biological factors and material circumstances such as living conditions. These intermediary determinants act as a pathway through which socioeconomic position influences health. The framework posits that differences in material circumstances such as living standards result in health inequalities. Economically deprived individuals are exposed to health-compromising conditions which result in them more frequently experiencing worse health outcomes than the more privileged individuals.

Based on the CSDH model and the data available in our dataset, the structural variables in our model included gender, age, residence, race, household income, education and employment status. Age was measured in years (from 15 years) and was included as a continuous variable. Place of residence was included as a dichotomous variable with $0-$ urban and $1-$ rural. The SANHANES collected ethnicity data on the categories of African, white, coloured, Indian/Asian and 'other'. These categories were based on the predominant ethnic groups in the 2001 census and are commonly included in analysis to assess inequalities due to the legacy of the pre 1994 racial hierarchy that existed in the country. None of the respondents declared 'other', therefore race was included as a categorical variable with 1 - African, 2 - White, 3 Coloured and 4 - Indian/Asian. The SANHANES-1 survey collected annual income data by asking each adult household member which income category best described their individual gross annual income. We then calculated the midpoint estimate of each category and used this to derive the total gross annual income per household, which was then divided by the number of adults in each household in order to get average income per household. This was then re-categorised into quantiles ranging from low to high income. Education was measured in years of schooling and was categorised as 0 - no education, 1 - primary (1-7 years), 2 secondary (8-12 years) and 3 -tertiary (13+ years). Employment was included as a categorical variable; 0 - unemployed, 1 -informal employment and 2 -formal employment.

The intermediary variables included BMI, self-rated health, smoking status, alcohol consumption and physical activity. BMI was a continuous variable calculated as weight divided by height squared. It was then recoded into a categorical variable as 0 - underweight (BMI<18.5), 1 - normal weight (BMI >=18.5 <25), 2 - overweight $(\mathrm{BMI}>=25<30)$ and $3-$ obese $(B M I \geq 30)$. Self-rated health was included as a categorical variable as $0-$ bad, $1-$ moderate and 2 - good. Respondents in the SANHANES-1 survey were asked if they smoked 
tobacco. This was categorised into 0 - never, 1 - occasional and 2 - regularly. Alcohol consumption was also included as a categorical variable with 0 - never, 1 - occasional and 2 - regularly. Respondents were asked whether they took part in some form of vigorous intensity sport, fitness or recreational activities such as running or weightlifting that cause large increases in the heart rate for at least 10 minutes at a time. This was included as a binary variable 0 - no 1 - yes.

\subsubsection{Data Analysis}

In SANHANES-1 self-reported diabetes was only investigated in the adult questionnaire (individuals aged 15 and older). Observations with missing data on comorbidity were excluded ( $n=6351$ ). The final sample used in the analysis was $n=12$ 594. Statistical analyses were performed in STATA software version 14. In order to account for clustering and survey design effects, we used STATA's stratified multi-stage design command. Therefore, data were weighted to produce estimates that represent the country's socio-demographic profile, based on the 2001 population census. For categorical variables, data were expressed as counts and proportions; mean values +/- standard deviations were reported for continuous variables. A multinomial logistic regression was conducted to analyse the association between several indicators such as social, economic, demographic and health variables, and having diabetes without CVD comorbidity, or diabetes with CVD comorbidity; the reference category was no diabetes or CVD. Step 1 of the regression analysis was the univariate or unadjusted analysis; step 2 was a multivariable analysis, which included structural variables only; step 3 was a multivariable analysis, which included intermediary variables only and the final step included all the variables in a multivariable analysis. In the analysis $95 \%$ Confidence Intervals and odds ratios are reported.

\subsection{Results}

The prevalence of diabetes and CVD conditions is shown in Table 7-1. The sample consisted of 12594 individuals of which 1,000 (5\%) reported having diabetes. Of the 1,000 people who reported diabetes, approximately $73 \%$ had at least one of the CVD chronic illnesses included in the study, whilst $27 \%$ reported having diabetes and no CVD chronic illness included in the study. Approximately $2 \%$ of respondents with diabetes reported having all three CVD chronic 
conditions; namely stroke, heart disease and high blood pressure (Figure 7-1). The CVD comorbidities of high blood pressure, heart disease and stroke were more prevalent amongst people with diabetes compared to people not living with diabetes (Figure 7-2).

As shown in Table 7-1, the majority of the respondents with diabetes-CVD comorbidity were female (66\%). Most of the respondents with diabetes-CVD comorbidity lived in urban areas (75\%), were African (66\%), unemployed (62\%), obese (80\%), were in the medium income category (42\%) and had secondary education (44\%). About $43 \%$ were regular smokers, $4 \%$ consumed alcohol regularly, $16 \%$ reported having bad self-rated health and $96 \%$ did not take part in any vigorous physical activity. The mean age for diabetes-CVD comorbidity was 57 years (standard deviation - 12.3). 
Table 7-1. Prevalence of self-reported diabetes and diabetic comorbidity ( $N=12594)$

\begin{tabular}{|c|c|c|c|c|c|c|}
\hline \multirow{2}{*}{$\begin{array}{l}\text { Chronic Illnesses } \\
\text { Age: mean +/- std.dev }\end{array}$} & \multicolumn{2}{|c|}{$\begin{array}{c}\text { None } \\
(N=11594 ; 94 \%)\end{array}$} & \multicolumn{2}{|c|}{$\begin{array}{l}\text { Diabetes only } \\
(N=269 ; 2 \%)\end{array}$} & \multicolumn{2}{|c|}{$\begin{array}{c}\text { Diabetes + other } \\
(\mathrm{N}=731 ; 4 \%)\end{array}$} \\
\hline & 33 & 14.3 & 48 & 14.9 & 57 & 12.3 \\
\hline \multicolumn{7}{|l|}{ Gender } \\
\hline Male & 5163 & $50 \%$ & 101 & $45 \%$ & 225 & $34 \%$ \\
\hline Female & 6425 & $50 \%$ & 168 & $55 \%$ & 506 & $66 \%$ \\
\hline \multicolumn{7}{|l|}{ Residence } \\
\hline Urban & 7676 & $67 \%$ & 197 & $70 \%$ & 551 & $75 \%$ \\
\hline Rural & 3918 & $33 \%$ & 72 & $30 \%$ & 180 & $25 \%$ \\
\hline \multicolumn{7}{|l|}{ Race } \\
\hline African & 7873 & $79 \%$ & 140 & $65 \%$ & 387 & $66 \%$ \\
\hline White & 513 & $10 \%$ & 18 & $17 \%$ & 30 & $14 \%$ \\
\hline Coloured & 2230 & $8 \%$ & 55 & $11 \%$ & 176 & $13 \%$ \\
\hline Indian & 938 & $2 \%$ & 56 & $7 \%$ & 136 & $7 \%$ \\
\hline \multicolumn{7}{|l|}{ Household income } \\
\hline Low & 3884 & $33 \%$ & 82 & $31 \%$ & 215 & $29 \%$ \\
\hline Medium & 4469 & $36 \%$ & 105 & $34 \%$ & 314 & $42 \%$ \\
\hline High & 3234 & $31 \%$ & 82 & $35 \%$ & 202 & $29 \%$ \\
\hline \multicolumn{7}{|l|}{ Education } \\
\hline None & 628 & $5 \%$ & 20 & $6 \%$ & 78 & $10 \%$ \\
\hline Primary & 1588 & $13 \%$ & 53 & $17 \%$ & 186 & $26 \%$ \\
\hline Secondary & 6615 & $67 \%$ & 132 & $63 \%$ & 291 & $44 \%$ \\
\hline Tertiary & 1084 & $15 \%$ & 28 & $14 \%$ & 71 & $19 \%$ \\
\hline \multicolumn{7}{|l|}{ Employment } \\
\hline Unemployed & 6752 & $58 \%$ & 131 & $54 \%$ & 395 & $62 \%$ \\
\hline Informal & 1201 & $10 \%$ & 58 & $22 \%$ & 165 & $17 \%$ \\
\hline Formal & 3393 & $32 \%$ & 72 & $23 \%$ & 148 & $21 \%$ \\
\hline \multicolumn{7}{|l|}{ Obesity } \\
\hline Underweight & 462 & $3 \%$ & 6 & $2 \%$ & 14 & $1 \%$ \\
\hline Normal Weight & 2166 & $17 \%$ & 29 & $12 \%$ & 54 & $8 \%$ \\
\hline Overweight & 977 & $8 \%$ & 41 & $16 \%$ & 91 & $11 \%$ \\
\hline Obese & 7989 & $72 \%$ & 193 & $69 \%$ & 572 & $80 \%$ \\
\hline \multicolumn{7}{|l|}{ Self-rated health } \\
\hline Bad & 414 & $3 \%$ & 20 & $8 \%$ & 111 & $16 \%$ \\
\hline Moderate & 1618 & $13 \%$ & 80 & $32 \%$ & 262 & $33 \%$ \\
\hline Good & 9341 & $83 \%$ & 165 & $60 \%$ & 348 & $50 \%$ \\
\hline \multicolumn{7}{|l|}{ Smoking } \\
\hline Never & 317 & $15 \%$ & 16 & $29 \%$ & 62 & $53 \%$ \\
\hline Occasional & 188 & $9 \%$ & 3 & $4 \%$ & 11 & $4 \%$ \\
\hline Regular & 1907 & $76 \%$ & 53 & $67 \%$ & 85 & $43 \%$ \\
\hline \multicolumn{7}{|l|}{ Alcohol } \\
\hline Never & 8442 & $72 \%$ & 211 & $76 \%$ & 603 & $80 \%$ \\
\hline Occasional & 2127 & $19 \%$ & 43 & $23 \%$ & 88 & $15 \%$ \\
\hline Regular & 782 & $9 \%$ & 5 & $1 \%$ & 31 & $4 \%$ \\
\hline \multicolumn{7}{|l|}{ Physical Activity } \\
\hline No & 9775 & $84 \%$ & 233 & $82 \%$ & 682 & $96 \%$ \\
\hline Yes & 1618 & $16 \%$ & 28 & $18 \%$ & 36 & $4 \%$ \\
\hline
\end{tabular}

Note - Percentage sign (\%) is mentioned where numbers and percentages are given; mean and standard deviation is mentioned for age; $\mathrm{n}$ : number of observations; std dev: standard deviation. Values are weighted. 
Figure 7-1 Cardiovascular comorbid conditions in people with diabetes

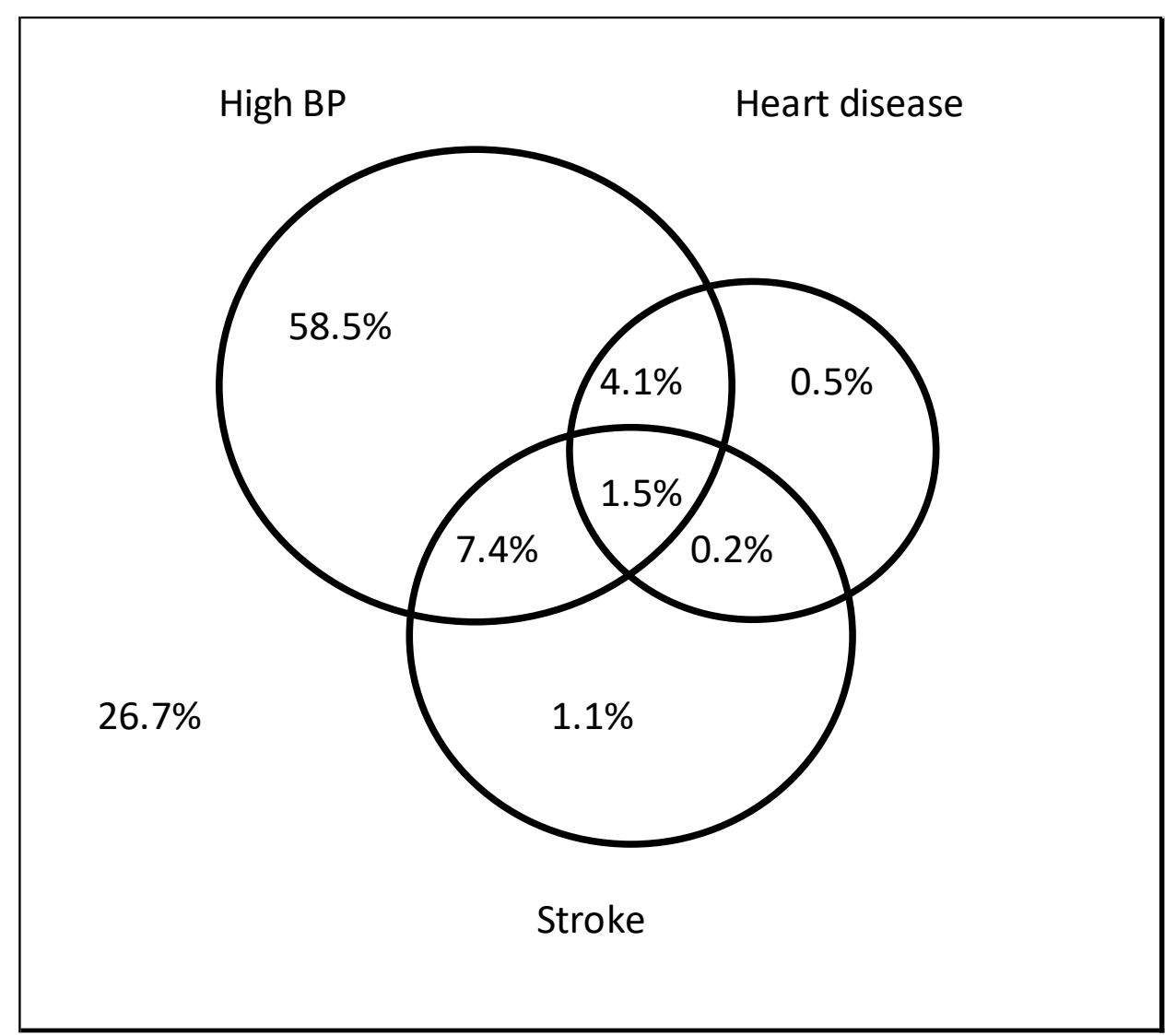

Figure 7-2 Prevalence of self-reported cardiovascular comorbidities among people with diabetes versus people without diabetes

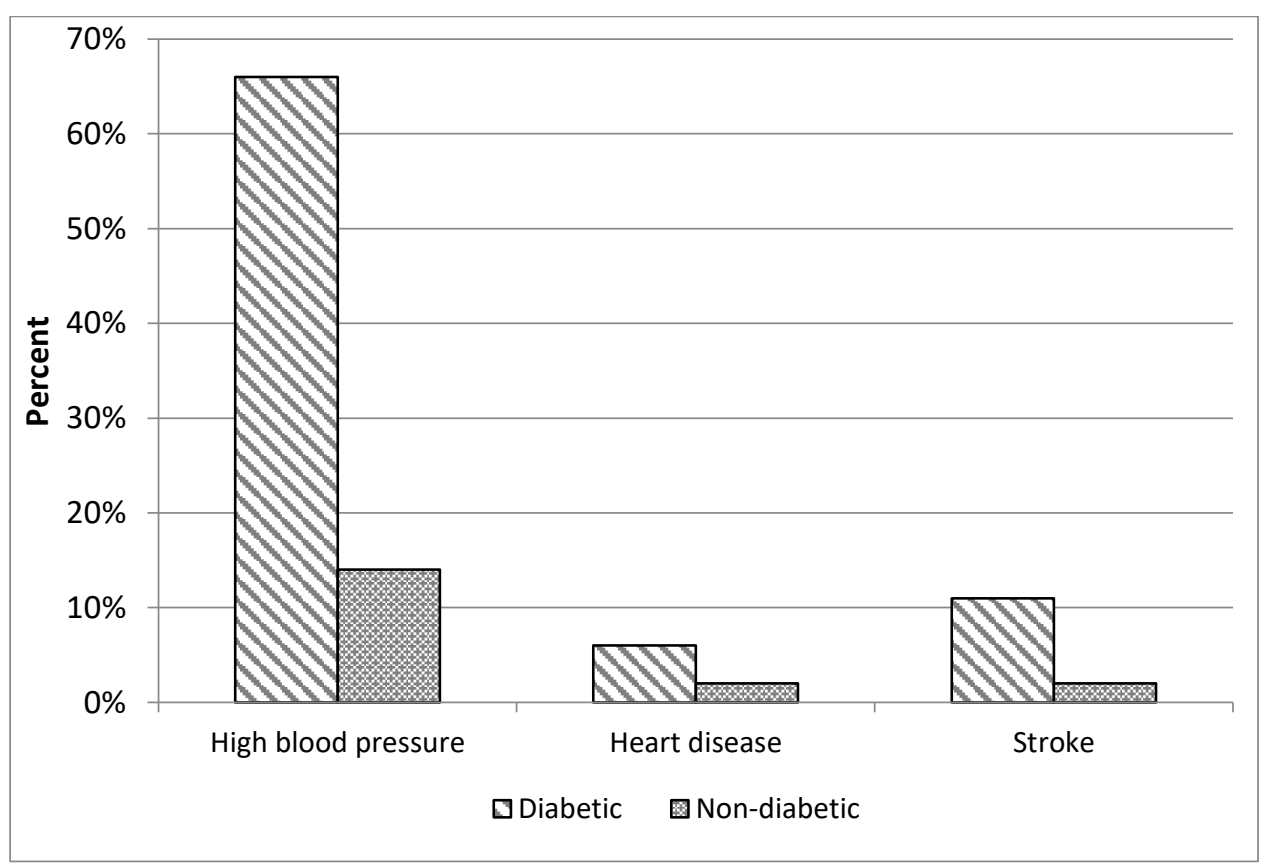


Table 7-2 and Table 7-3 show results from the multinomial logistic regression analysis, using those with no diabetic chronic illness as the reference category. Table 7-2 shows the results for the unadjusted and adjusted association of each variable with diabetes without CVD and Table 7-3 shows the results for the unadjusted and adjusted association of each variable with diabetes and CVD comorbidity (diabetes plus one or more CVD chronic illnesses). In step 1, the univariate model for respondents with diabetes without CVDs, age (Odds ratio [OR] 1.06; 95\% Confidence Interval 1.05-1.07), being coloured $(1.68 ; 1.13-2.50)$ or Indian $(3.65 ; 2.09$ 6.36) versus African, having informal employment (2.41; 1.25-4.66) versus unemployed were positively associated with having diabetes only, whilst good self-rated health $(0.31 ; 0.17-0.56)$ versus bad self-rated health and regular alcohol consumption $(0.11 ; 0.04-0.33)$ were negatively associated with having diabetes only, with statistically significant associations. In the univariate model for patients with diabetes and CVD comorbidity, all variables except income were significantly associated with this comorbidity.

In step 2 (structural factors models), the variables significantly associated with having diabetes only were age $(1.06 ; 1.04-1.07)$, Indian $(2.93 ; 1.57-5.49)$ versus African and secondary education $(2.39 ; 1.18-4.84)$ versus no education. The variables significantly associated with diabetes and CVD comorbidity were age $(1.1 ; 1.09-1.11)$, female $(2.03 ; 1.44-$ $2.86)$ versus males, rural residence $(0.59 ; 0.42-0.81)$ versus urban residence, being Coloured (1.38; $1.01-1.89)$ or Indian $(2.15 ; 1.25-3.70)$ versus being black and primary $(2.4 ; 1.40-4.12)$, secondary $(2.32 ; 1.29-4.18)$ or tertiary education $(3.52 ; 1.55-7.99)$ versus no education. Rural residence was the only variable negatively associated with diabetes-CVD comorbidity implying that those who lived in rural areas were less likely to report this comorbidity.

In step 3 (intermediary factors models), being an occasional smoker $(0.04 ; 0.01-0.22)$ versus not smoking, being an occasional $(0.27 ; 0.13-0.57)$ or regular $(0.04 ; 0.01-0.24)$ alcohol drinker versus not consuming alcohol were the variables associated with having diabetes only. All variables in step 3 were significantly associated with diabetes-CVD comorbidity. A negative relationship was observed for good self-rated health $(0.15 ; 0.07-0.36)$ versus bad self-rated health, occasional $(0.18 ; 0.06-0.52)$ and regular $(0.21 ; 0.10-0.46)$ smokers versus nonsmokers, occasional $(0.41 ; 0.20-0.83)$ and regular $(0.20 ; 0.10-0.47)$ alcohol drinkers versus not 
consuming alcohol and physical activity $(0.07 ; 0.02-0.27)$. This pattern was similar to that observed in the univariate model for the comorbidity studied.

In step 4, the full model, after adjusting for both structural and intermediary factors, age (1.06; 1.03-1.08), being Indian (3.49; 1.11-10.96) versus being African occasional smokers $(0.03 ; 0.00-0.26)$ versus non-smokers and occasional $(0.31 ; 0.13-0.76)$ or regular drinkers (0.05; 0.01-0.36) versus no alcohol consumption were the only variables significantly associated with diabetes only. Except for age and being Indian, all other variables mentioned above were negatively associated with diabetes only. Variables significantly associated with diabetes-CVD comorbidity were age $(1.09 ; 1.06-1.12)$, high income $(0.27 ; 0.10-0.76)$ versus low-income, moderate $(0.33 ; 0.11-0.96)$ and good self-rated health $(0.24 ; 0.08-0.68)$ versus bad self-rated health, occasional $(0.29 ; 0.10-0.88)$ and regular smokers $(0.25 ; 0.12-0.53)$ versus non-smokers and physical activity $(0.15 ; 0.03-0.68)$. Age was the only variable positively associated with diabetes-CVD comorbidity. The adjusted model was used to control for potentially confounding variables. It is partly for this reason that we find a noticeable number of non-significant results in the final model (step 4) in comparison to the adjusted model (step 1). 
Table 7-2 Logistic regression analyses of factors associated with diabetes without CVD comorbidity, no diabetes or CVD is the reference category

\begin{tabular}{|c|c|c|c|c|c|c|c|c|}
\hline \multirow[b]{2}{*}{ Diabetes Only } & \multicolumn{2}{|c|}{ Step 1-Unadjusted } & \multicolumn{2}{|c|}{ Step 2-Structural } & \multicolumn{2}{|c|}{ Step 3-Intermediary } & \multicolumn{2}{|c|}{ Step 4-Full } \\
\hline & OR & $(95 \% \mathrm{Cl})$ & OR & $(95 \% \mathrm{Cl})$ & OR & $(95 \% \mathrm{Cl})$ & OR & $(95 \% \mathrm{Cl})$ \\
\hline Age & $1.06 * *$ & $(1.05-1.07)$ & $1.06 * *$ & $(1.04-1.07)$ & & & $1.06 * *$ & $(1.03-1.08)$ \\
\hline \multicolumn{9}{|l|}{ Gender } \\
\hline Male & 1 & & 1 & & & & 1 & \\
\hline Female & 1.22 & $(0.81-1.85)$ & 1.07 & $(0.63-1.83)$ & & & 0.8 & $(0.34-1.84)$ \\
\hline \multicolumn{9}{|l|}{ Residence } \\
\hline Urban & 1 & & 1 & & & & 1 & \\
\hline Rural & 0.88 & $(0.61-1.27)$ & 0.98 & $(0.63-1.53)$ & & & 1 & $(0.32-3.00)$ \\
\hline \multicolumn{9}{|l|}{ Race } \\
\hline African & 1 & & 1 & & & & 1 & \\
\hline White & 2.05 & $(0.92-4.60)$ & 1.29 & $(0.45-3.66)$ & & & 1.05 & $(0.20-5.36)$ \\
\hline Coloured & $1.68 * *$ & $(1.13-2.50)$ & 1.61 & $(0.99-2.62)$ & & & 1.58 & $(0.64-3.95)$ \\
\hline Indian & $3.65^{* *}$ & $(2.09-6.36)$ & $2.93 * *$ & $(1.57-5.49)$ & & & $3.49 *$ & $(1.11-10.96)$ \\
\hline \multicolumn{9}{|l|}{ Household income } \\
\hline Low & 1 & & 1 & & & & 1 & \\
\hline Medium & 0.99 & $(0.66-1.51)$ & 0.73 & $(0.46-1.15)$ & & & 0.93 & $(0.36-2.40)$ \\
\hline High & 1.19 & $(0.71-2.02)$ & 0.78 & $(0.45-1.35)$ & & & 0.47 & $(0.16-1.32)$ \\
\hline \multicolumn{9}{|l|}{ Education } \\
\hline None & 1 & & 1 & & & & 1 & \\
\hline Primary & 0.97 & $(0.51-1.83)$ & 1.76 & $(0.87-3.53)$ & & & 1.91 & $(0.36-10.14)$ \\
\hline Secondary & 0.70 & $(0.38-1.27)$ & $2.39 * *$ & $(1.18-4.84)$ & & & 4.51 & $(0.77-26.47)$ \\
\hline Tertiary & 0.71 & $(0.34-1.51)$ & 1.65 & $(0.51-5.30)$ & & & 5.57 & $(0.70-44.97)$ \\
\hline \multicolumn{9}{|l|}{ Employment } \\
\hline Unemployed & 1 & & 1 & & & & 1 & \\
\hline Informal & $2.41 * *$ & $(1.25-4.66)$ & 1.41 & $(0.68-2.90)$ & & & 0.79 & (0.31-1.98) \\
\hline Formal & 0.79 & $(0.53-1.17)$ & 0.71 & $(0.44-1.13)$ & & & 0.90 & $(0.36-2.21)$ \\
\hline \multicolumn{9}{|l|}{ Obesity } \\
\hline Underweight & 1 & & & & 1 & & & \\
\hline Normal Weight & 0.96 & $(0.30-3.10)$ & & & 0.65 & $(0.13-3.39)$ & 0.42 & $(0.07-2.67)$ \\
\hline Overweight & 2.84 & $(0.91-8.90)$ & & & 2.63 & $(0.46-14.97)$ & 0.90 & $(0.12-6.44)$ \\
\hline Obese & 1.29 & $(0.44-3.80)$ & & & 0.59 & $(0.13-2.67)$ & 0.35 & $(0.06-2.00)$ \\
\hline \multicolumn{9}{|l|}{ Self-rated health } \\
\hline Bad & 1 & & & & 1 & & 1 & \\
\hline Moderate & 1.02 & $(0.50-2.09)$ & & & 1.04 & $(0.28-3.94)$ & 1.34 & $(0.32-5.49)$ \\
\hline Good & $0.31 * *$ & $(0.17-0.56)$ & & & 0.52 & $(0.14-1.92)$ & 0.79 & $(0.17-3.60)$ \\
\hline \multicolumn{9}{|l|}{ Smoking } \\
\hline Never & 1 & & & & 1 & & 1 & \\
\hline Occasional & 0.27 & $(0.04-1.84)$ & & & $0.04 * *$ & $(0.01-0.22)$ & $0.03 * *$ & $(0.00-0.26)$ \\
\hline Regular & 0.47 & $(0.19-1.13)$ & & & 0.72 & $(0.32-1.61)$ & 1.02 & $(0.40-2.65)$ \\
\hline \multicolumn{9}{|l|}{ Alcohol } \\
\hline Never & 1 & & & & 1 & & 1 & \\
\hline Occasional & 1.13 & $(0.60-2.11)$ & & & $0.27 * *$ & $(0.13-0.57)$ & $0.31 * *$ & $(0.13-0.76)$ \\
\hline Regular & $0.11 * *$ & $(0.04-0.33)$ & & & $0.04 * *$ & $(0.01-0.24)$ & $0.05 * *$ & $(0.01-0.36)$ \\
\hline \multicolumn{9}{|l|}{ Physical Activity } \\
\hline No & 1 & & & & 1 & & 1 & \\
\hline Yes & 1.18 & $(0.55-2.53)$ & & & 0.93 & $(0.35-2.46)$ & 1.62 & $(0.52-5.05)$ \\
\hline
\end{tabular}

${ }^{* *} p<0.001 ;{ }^{*} p<0.05 ;$ OR- Odds ratio; 95\% Confidence Interval; CVD-Cardiovascular disease 
Table 7-3 Logistic regression analyses of factors associated with diabetes-CVD comorbidity, no diabetes or CVD is the reference category

\begin{tabular}{|c|c|c|c|c|c|c|c|c|}
\hline $\begin{array}{l}\text { Diabetes with CVDs } \\
\text { comorbidity }\end{array}$ & \multicolumn{2}{|c|}{ Step 1-Unadjusted } & \multicolumn{2}{|c|}{ Step 2-Structural } & \multicolumn{2}{|c|}{ Step 3-Intermediary } & \multicolumn{2}{|c|}{ Step 4-Full } \\
\hline \multicolumn{9}{|l|}{ Gender } \\
\hline Male & 1 & & 1 & & & & 1 & \\
\hline Female & $1.96 * *$ & (1.49-2.59) & $2.03 * *$ & $(1.44-2.86)$ & & & 1.43 & $(0.76-2.68)$ \\
\hline Urban & 1 & & 1 & & & & 1 & \\
\hline Rural & $0.68 * *$ & $(0.53-0.86)$ & $0.59 * *$ & $(0.42-0.81)$ & & & 0.44 & $(0.18-1.06)$ \\
\hline \multicolumn{9}{|l|}{ Race } \\
\hline African & 1 & & 1 & & & & 1 & \\
\hline White & 1.71 & $(0.85-3.42)$ & 0.56 & $(0.26-1.23)$ & & & 1.62 & $(0.54-4.84)$ \\
\hline Coloured & $1.82 * *$ & $(1.45-2.29)$ & $1.38^{*}$ & (1.01-1.89) & & & 1.94 & $(0.92-4.11)$ \\
\hline Medium & 1.3 & $(0.90-1.89)$ & 0.95 & $(0.63-1.43)$ & & & 0.58 & $(0.28-1.17)$ \\
\hline High & 1.07 & $(0.71-1.60)$ & 0.73 & (0.39-1.36) & & & $0.27 *$ & $(0.10-0.76)$ \\
\hline \multicolumn{9}{|l|}{ Education } \\
\hline None & 1 & & 1 & & & & 1 & \\
\hline Primary & 0.91 & $(0.62-1.34)$ & $2.4^{* *}$ & $(1.40-4.12)$ & & & 1.17 & $(0.40-3.48)$ \\
\hline Secondary & $0.30 * *$ & $(0.20-0.43)$ & $2.32 * *$ & $(1.29-4.18)$ & & & 2.55 & $(0.78-8.33)$ \\
\hline Tertiary & 0.57 & $(0.29-1.12)$ & $3.52 * *$ & $(1.55-7.99)$ & & & 3.33 & $(0.79-14.13)$ \\
\hline \multicolumn{9}{|l|}{ Employment } \\
\hline Unemployed & 1 & & 1 & & & & 1 & \\
\hline Informal & $1.67 * *$ & $(1.21-2.30)$ & 0.94 & $(0.62-1.43)$ & & & 0.86 & $(0.30-2.48)$ \\
\hline Formal & $0.62 * *$ & $(0.45-0.85)$ & 0.87 & $(0.58-1.30)$ & & & 0.71 & (0.34-1.51) \\
\hline Moderate & $0.52 * *$ & $(0.37-0.75)$ & & & 0.23 & $(0.10-0.54)$ & $0.33^{*}$ & $(0.11-0.96)$ \\
\hline Good & $0.13^{* *}$ & (0.09-0.19) & & & $0.15 * *$ & $(0.07-0.36)$ & $0.24 * *$ & $(0.08-0.68)$ \\
\hline \multicolumn{9}{|l|}{ Smoking } \\
\hline Never & 1 & & & & 1 & & 1 & \\
\hline Occasional & $0.14^{* *}$ & $(0.05-0.40)$ & & & $0.18 * *$ & $(0.06-0.52)$ & $0.29 *$ & (0.10-0.88) \\
\hline Regular & $0.16 * *$ & $(0.07-0.36)$ & & & $0.21 * *$ & $(0.10-0.46)$ & $0.25 * *$ & $(0.12-0.53)$ \\
\hline \multicolumn{9}{|l|}{ Alcohol } \\
\hline Never & 1 & & & & 1 & & 1 & \\
\hline Occasional & 0.71 & $(0.49-1.02)$ & & & $0.41^{*}$ & $(0.20-0.83)$ & 0.66 & $(0.30-1.41)$ \\
\hline Regular & $0.45^{* *}$ & $(0.27-0.75)$ & & & $0.20 * *$ & $(0.10-0.47)$ & 0.35 & (0.11-1.10) \\
\hline \multicolumn{9}{|l|}{ Physical Activity } \\
\hline No & 1 & & & & 1 & & 1 & \\
\hline Yes & $0.23 * *$ & $(0.14-0.37)$ & & & $0.07 * *$ & $(0.02-0.27)$ & $0.15^{*}$ & $(0.03-0.68)$ \\
\hline
\end{tabular}

${ }^{* *} \mathrm{p}<0.001 ;{ }^{*} \mathrm{p}<0.05$; OR-Odds Ratio; 95\% Confidence Interval; CVD-Cardiovascular disease 


\subsection{Discussion}

Previous studies have reported on the elevated risk of comorbidities in people with diabetes (IDF, 2001; André Pascal Kengne, Amoah, \& Mbanya, 2005; Mo, Pogany, Li, \& Morrison, 2006; WHO, 2016a). However not much is reported on the association between diabetes CVD comorbidity and various behavioural and social determinants. This study is thus important as it adds to the literature on diabetes in South Africa and Sub-Saharan Africa in general. Our study finds that high blood pressure, heart disease and stroke were more prevalent amongst people with diabetes compared to people without diabetes. This finding supports the assertion that the risk of CVDs in people with diabetes is more than double that of individuals without diabetes (IDF, 2001). Similar to other studies, we find that high blood pressure was the most common comorbid CVD condition among people with diabetes, followed by heart disease and stroke (Mo et al., 2006). Comorbidity of diabetes and high blood pressure has been found to be high in previous studies (Folb et al., 2015; André Pascal Kengne et al., 2005; Andre Pascal Kengne, Dzudie, \& Sobngwi, 2008). This is of great concern given the increase in costs associated with the management of complicated diabetes.

The estimates of diabetes CVD presented provide only a snapshot of the complications commonly reported in people with diabetes. In our study both diabetes and diabetes CVD comorbidity is more prevalent among individuals with higher education levels (secondary and tertiary). This finding is contrary to that observed in European countries where diabetes is more prevalent among the lower education groups (Dalstra et al., 2005). It should be noted however that the data observed in South Africa may not be directly comparable to other countries because the data presented were self-reported and might be subject to social desirability bias and reporting bias. As the symptoms of diabetes are not always correctly interpreted, the self-reported incidences may underreport the true prevalence of diabetes. Also given that the met need for diabetes diagnosis increases with education (Manne-Goehler et al., 2016) it is also possible that less educated individuals have not yet been diagnosed. Consistent with other studies we find that diabetes and diabetes CVD comorbidity was more common in those who reside in urban areas (Motala \& Ramaiya, 2010). This is perhaps a result of sedentary lifestyle, poor diets and rapid urbanisation. 
Significant associations were observed for the structural variables of age, race and income. Our findings are consistent with literature that found that the occurrence of two or more chronic illnesses is associated with age (Alaba \& Chola, 2013; Mo et al., 2006; PhaswanaMafuya et al., 2013). Diabetes and its comorbid conditions is shown to be very common amongst the elderly (Dybicz, Thompson, Molotsky, \& Stuart, 2011; Zhang et al., 2010). In our study, the mean age of having diabetes and CVD comorbidity was 48 years and 57 years, respectively. Although the occurrence of two or more chronic illnesses is common amongst the elderly, recent studies have shown that it is not only limited to the elderly (Agborsangaya, Lau, Lahtinen, Cooke, \& Johnson, 2012; Barnett et al., 2012). A positive association was observed between being Indian and having diabetes, even after adjusting for other variables. This association was however not observed in individuals with diabetes and CVD comorbidity. This relationship has also been observed and discussed in other reports that find that although Indians have a strong genetic predisposition for diabetes, the prevalence of diabetes complications is not associated with any race in particular (Motala \& Ramaiya, 2010). Our finding that income is not associated with having diabetes only (without CVDs) is consistent with a South African population based cohort study that found no significant association between diabetes and income (Egbujie, Igumbor, \& Puoane, 2016). This finding is contrary to that presented in a global systematic review which finds that individuals in low-income groups are at an increased risk of diabetes (Agardh, Allebeck, Hallqvist, Moradi, \& Sidorchuk, 2011). Whilst the authors find this relationship to be consistently strong in high income countries, they do not find a consistently strong relationship in LMICs and call for further investigations (Agardh et al., 2011). Our data however indicates that persons with high income were less likely to report having diabetes-CVD comorbidity when compared to individuals with lowincome. High income could reflect better access and use of health care facilities and adoption of good diabetes management practices. This finding warrants further investigation into the income differentials and the effects of socio-economic gradients in diabetes CVD comorbidity.

Various intermediary factors were also found to be associated with either diabetes only or diabetes CVD comorbidity. Empirical studies indicate that exercise interventions are associated with decreases in the incidence of diabetes (Xiao-Ren, Guang-Wei, Ying-Hua, JiXing, \& et al., 1997) and that physical inactivity is associated with diabetes (Sobngwi et al., 2002). However, our study finds an insignificant association between diabetes and physical 
activity. The differences in the nature of association between diabetes and physical activity could be a result of variations in definitions and measurements of physical activity. Our study included leisure time physical activity whilst other studies include both leisure time and occupation physical activity (Kodama et al., 2013; Sobngwi et al., 2002). Data on physical activity was collected via patient interview and is therefore subject to social desirability bias. Therefore, our estimates may lack precision. However, we do find that physical activity is negatively associated with diabetes-CVD comorbidity, implying that those who took part in physical activity were less likely to report diabetes and CVDs comorbidity when compared to those who did not take part in any physical activity. This result is consistent with studies that find that physical activity is associated with reduced risk of CVDs in people with diabetes (Kodama et al., 2013). Smoking is also a well-known risk factor of NCDs such as diabetes (National Department of Health, 2013; Mo et al., 2006). In our study surprisingly, being an occasional or regular smoker was negatively associated with diabetes-CVD comorbidity. Using the South African National Income Dynamics Survey data, Alaba and Chola (2013) also find that smoking is negatively associated with the occurrence of two or more chronic conditions in an individual (Alaba \& Chola, 2013). This unexpected finding calls for further investigations. We however note that caution needs to be observed when interpreting this finding because data on smoking was self-reported and may be subject to social desirability bias. As expected, we found that those who reported moderate and good self-rated health were less likely to report diabetes-CVD comorbidity. This is in line with other studies which found that the presence of multiple chronic illnesses is associated with poor perceived health (Fortin, Dubois, Hudon, Soubhi, \& Almirall, 2007).

Diabetes and CVD comorbidity presents a major challenge to health care management and has the potential to increase costs yet little is known about the trajectories for diabetes complications in South Africa. This study, within its limitations, strengthens the evidence base on the magnitude of diabetes and its comorbid CVD conditions. The identification of the factors associated with diabetes and its comorbid CVD conditions provides useful information that can be used for policy refinement. For example, the finding that high income is negatively associated with diabetes-CVD comorbidity might point to the fact that addressing income inequalities may be essential to reducing diabetes-CVD comorbidity. Encouraging physical activity in people with diabetes may also help in reducing diabetes-CVD comorbidity. 
Notwithstanding, there is still a need for a better understanding of the underlying causes of comorbidities between diabetes and other chronic diseases such as neuropathy and nephropathy. The National Department of Health strategic plan for the prevention and control of NCDs emphasises the need to strengthen health care systems by ensuring that chronic disease management is integrated (National Department of Health, 2013). The plan sets out to reduce the risk factors associated with NCDs and also increase the percentage of people controlled for diabetes by 2030. In order to influence the plan and or policy, further research into the patterns and implications of diabetes and chronic communicable disease comorbidity is imperative. While our study does not address issues of communicable diseases among persons with diabetes, we identify other common chronic comorbid conditions which should be taken into consideration when designing interventions aimed at integrated management of chronic diseases.

Follow up surveys are crucial in monitoring trends and patterns of diabetes comorbidity and will form an ideal conduit for further research and policy refinement. Given the need for fiscal prudence that characterises the South African economy, addressing diabetes and its comorbid conditions through the targeting of individuals with multiple risk factors is crucial.

\subsection{Limitations}

This study used nationally representative data to determine the prevalence of diabetes-CVD comorbidity and examine the factors associated with this comorbidity at the time of the survey. The study considered three potential diabetes-CVD comorbid conditions that were reported in the dataset, heart disease, high blood pressure and stroke. However, diabetes may be associated with various other comorbid conditions such as depression, osteoarthritis, neuropathy, nephropathy and HIV. We were unable to assess total comorbidity. It is possible that an individual with diabetes might have been misclassified as having no other illnesses when in actual fact they were diagnosed with other conditions not reported in the data. This limitation is also exacerbated by the fact that the presence of chronic disease was selfreported and not measured by a health care professional. It is possible that some individuals suffered from undiagnosed chronic illnesses resulting in further underreporting. The lack of population level data on diabetes unmet care was reported in one recent study by Manne- 
Goehler et al (2016), which revealed both a high prevalence and a large unmet need for diabetes care within sub-Saharan Africa (Manne-Goehler et al., 2016). It is possible that the prevalence of diabetes was underestimated and that there is a high unmet need for diabetes care in South Africa. Because diabetes was self-reported, we were also unable to classify the type of diabetes that was reported therefore our study included risk factors such as physical inactivity common to type 2 diabetes. It is important to note that the data used is this study is self-reported and is subject to social desirability bias. Due to the cross-sectional nature of the study, casual inferences cannot be drawn.

\subsection{Conclusion}

To our knowledge, this is the first study in South Africa to look at diabetes-CVD comorbidity and its association with various socio-economic, behavioural and demographic factors. Diabetes and its comorbid conditions potentially place a heavy burden on the health care system as a result of increased demand for health care. Studies like ours contribute to the current pool of knowledge about factors predisposing, promoting and establishing disease in patients with previously existing illness. Our study highlighted differences in the correlates of diabetes and CVD comorbidity. High income and physical activity were significantly associated with diabetes-CVD comorbidity. Evidence has shown that lifestyle habits such as physical activity are essential for the management of diabetes (WHO, 2016a). In our study, we also show that the elderly are at risk of having diabetes or diabetes-CVD comorbidity. Given the rapidly increasing size of the older population, it is critical to generate evidence on the magnitude of diabetic comorbidity in South Africa in order to inform the development of interventions for the prevention of diabetic comorbidity and strengthen the health care system. Health sector policy reforms such as the National Health Insurance (Department of Health, 2015a) should target vulnerable groups such as the elderly as they are at risk of chronic illnesses. The monitoring of diabetic comorbidity will guide research into the capabilities of primary health care workers in holistically managing comorbidity. Our study consistently showed that those who took part in any physical activity were less likely to report diabetes-CVD comorbidity. There is a need to put in place measures and interventions that encourage more active and healthy lifestyles. 


\section{Chapter 8}

General Discussions and concluding remarks 


\subsection{Introduction}

This dissertation is undertaken with the aim to evaluate the economic impact and inequalities in diabetes in South Africa. The key concepts of the dissertation are highlighted in the conceptual framework (Figure 1-4) presented in the introductory chapter of the dissertation. As outlined in Chapter 1 of the dissertation, the motivation behind this topic is the importance to reduce the burden of diabetes and to promote policies that ensure financial protection against health care costs and improved diabetes health outcomes. Studies have shown that the economic burden of diabetes is huge. This burden is particularly high in LMICs in which diabetes affects the working-age populations. The burden of diabetes on patients and families is further intensified by the use of OOP payments for health financing - a common practice in African countries - and the lack of sufficient resources for diabetes management. As previously noted in the conceptual framework of Chapter 1, diabetes is a chronic illness whose development may be influenced by various factors such as socio-economic status and lifestyle behaviours. The illness is better managed when diagnosed early, and when managed via various self-care practices such as medication adherence, frequent health check-ups, avoiding smoking and following a healthy diet. These diabetes management practices are essential to improving diabetes health outcomes as they may prevent or delay the development of diabetes complications (see Figure 1-4).

To meet its aim, the dissertation relies on 6 chapters that make use of two datasets: (1) a national survey called the 2012 SANHANES-1; (2) data we collected ourselves at three diabetes clinics in the Tshwane health district. Chapters 4 and 7 make use of the SANHANES1 dataset, chapters 3,5 and 6 make use of the data collected from the three diabetes clinics. A first step in addressing the dissertation objectives involves a review of the available evidence on the costs of diabetes in Africa. Data collected via desktop research in Chapter 2 reveal the huge diabetes burden in Africa and also the lack of empirical evidence on the impact of diabetes health care costs on individuals. On the basis of the evidence collected in the review and using data collected from the diabetes clinics, we assess the impact of health care costs by estimating two measures of financial protection, catastrophic health expenditure and impoverishment in Chapter 3. Given the huge income inequalities in South Africa, in Chapter 4 the dissertation assesses the socio-economic inequalities in diabetes in 
South Africa and investigates the contribution of lifestyle factors to diabetes inequalities. Lifestyle factors are not only important for the prevention of diabetes but also in diabetes management. Evidence on the burden of diabetes would be incomplete without considering how patients cope with diabetes management. In Chapter 5, we assess the level of and inequalities in diabetes self-care behaviours such as lifestyle behaviours. Building on this analysis qualitative data are used in Chapter 6 to explore the factors that promote or inhibit adherence to self-care practices, a relation that is described in the dissertation's conceptual framework (Chapter 1). Given that non-adherence to self-care is linked to the development of complications, what is the prevalence of and factors associated with diabetes CVD comorbidity in South Africa? Chapter 7 focuses on addressing this question. Specifically, we investigate diabetes CVD comorbidity in South Africa.

Our study also has limitations. These are discussed in each of the chapters $2-7$. Whilst the broad range of evidence presented in this dissertation, contributes to our knowledge on diabetes in South Africa, there is still a need for further research, in particular research that explores the economic impact of diabetes on patients visiting private health care facilities that make use of medical insurance and studies that make use of national datasets to assess diabetes self-care practices in South Africa. In this chapter, we present the key findings of the dissertation in the form of statements and discuss each in turn. Conclusions on areas for future research and policy implications complete each discussion section.

\subsection{Main findings}

Statement 1: In South Africa, differences in socio-economic inequalities exist in terms of diabetes prevalence, diabetes health care costs and diabetes self-care practices

As we see in Chapter 3, 4 and 5, socio-economic inequalities exist in terms of diabetes prevalence, the cost of diabetes care and diabetes self-care practices. The introductory chapter also highlights the huge income inequalities evident in South Africa. Despite efforts to stimulate inclusive growth, the income inequalities continue to grow. The country is reported to have one of the highest income inequalites in the World (Sulla \& Zikhali, 2018). High income inequalities are intertwined with high health inequalities (Elgar, 2010). 
Underlying inequalities in predisposing factors or social determinants of health, are recognised as the driving force behind health inequalities. This dissertation addresses objectives 2, 3 and 4 by making use of concentration indices to estimate socio-economic inequalities in the distribution of diabetes, diabetes health care costs and diabetes self-care practices.

Using data from the SANHANES-1, Chapter 4 reveals the presence of socio-economic inequalities in the distribution of diabetes. Consistent with other studies, we also document the high levels of undiagnosed diabetes within South Africa (Stokes et al., 2017). Given this huge prevalence of undiagnosed diabetes in South Africa and making use of diagnosed and undiagnosed diabetes estimates, we find that diabetes is concentrated amongst those within higher socio-economic groups. Our finding corroborates other related literature that finds a pro-rich distribution of diabetes in South Africa when using self-reported data only (Ataguba, Akazili, \& Mclntyre, 2011; Mukong, Van Walbeek, \& Ross, 2017). Given the importance of lifestyle factors in the prevention of diabetes, the study also assesses the role of lifestyle factors in the socio-economic distribution of diabetes using a decomposition framework proposed by Wagstaff, Doorslaer and Watanabe (2001). For a variable to contribute to inequalities in diabetes, it needs to be related to diabetes and it also needs to be unequally distributed across socio-economic groups. Our study shows the presence of socio-economic inequalities in lifestyle factors, such as smoking, alcohol consumption, physical activity, obesity, vegetable and fruit consumption, which in turn contribute to socio-economic inequalities in diabetes. The importance of lifestyle factors and their contribution to inequalities in health and diabetes specifically, is also supported by other studies (Agardh et al., 2004; Borg \& Kristensen, 2000; Stringhini et al., 2012). These findings point to the importance of lifestyle factors when addressing inequalities in health.

Our findings in Chapter 3 show inequalities in catastrophic health expenditure due to diabetes that favour the rich. This means the prevalence of catastrophic health expenditure due to diabetes is more concentrated amongst those within the low socio-economic status groups. In South African public hospital health care is provided according to the UPFS which groups patients into full paying or subsidised patients. Fully subsided patients do not need to pay OOP for health care whilst partially subsidised pay according to their income level. Our finding 
on the concentration of catastrophic health expenditure amongst those within the low socioeconomic status groups, points to how ineffective the existing UPFS has been in protecting people with diabetes in low socio-economic groups from financial hardship. This finding is consistent with growing South African literature that finds a high health care cost burden within poorer socio-economic groups (Goudge, Gilson, Russell, Gumede, \& Mills, 2009). The finding is also consistent with a study amongst people with diabetes visiting a hospital in Nigeria (Okoronkwo, Ekpemiro, Okwor, Okpala, \& Adeyemo, 2015). Also, previous reviews on catastrophic health expenditure in sub-Saharan Africa point to the vulnerability of the poor to catastrophic health expenditure (Njagi, Arsenijevic, \& Groot, 2018). As established in the introductory chapter, in South Africa, those of lower socio-economic status primarily make use of public health care services. Thus, despite the modest charges within public hospitals, the poor are at an increased risk of catastrophic health expenditure because for the poor even a small amount of health cost can be catastrophic.

Evidence from Chapter 5 shows that differences in socio-economic status amongst people with diabetes, lead to differing abilities to cope and adhere to self-care practices. As discussed in the introductory chapter, diabetes is best managed when patients adhere to recommended self-care practices. In Chapter 5 for example, using evidence from the concentration indices, we find that dietary diversity is more concentrated amongst those of higher socio-economic status (see Table 5-4 in Chapter 5). This finding is consistent with literature that shows that higher income levels are associated with dietary diversity (Tiew, Chan, Lye, \& Loke, 2014). These findings are also supported by evidence from the regression analysis in the same chapter where we find that dietary diversity is associated with a higher wealth index. We also find that physical activity is more concentrated amongst those of higher socio-economic status (see Table 5-4 in Chapter 5). These findings are also supported by evidence from our regression analysis in the same chapter where we find that physical activity is associated with a higher wealth index. Our review of previous studies shows that there is no consistent direction in the socio-economic inequalities in physical activity. A systematic review on the socio-economic inequalities in various domains of physical activity using European studies, shows that the direction of inequality differs by the physical activity domain (Beenackers et al., 2012). Whilst occupational physical activity is more concentrated amongst those of lower socio-economic status, leisure time physical activity is more concentrated amongst those of 
higher socio-economic status (Beenackers et al., 2012). In our study we also find that adherence to not smoking is concentrated amongst those of lower socio-economic status. For diabetes populations, a London-based study finds that smoking is more concentrated amongst those within the high socio-economic groups (Gulliford, Sedgwick, \& Pearce, 2003). Studies on cigarette smoking in the general population, mostly indicate a higher concentration of smoking amongst those with lower socio-economic status, particularly in developed countries (Hiscock, Bauld, Amos, Fidler, \& Munafò, 2012). Although findings from developing countries also show a socio-economic gradient in smoking, the direction of inequality differs.

\section{Research and policy implications}

Some of the inequality estimates generated by our analysis, are not based on nationally representative data. In order to draw generalisations from the results, any future study should make use of nationally representative datasets to estimate socio-economic inequalities in diabetes health care cost and diabetes self-care practices. To facilitate robust over time analysis of diabetes inequalities, future research should make use of panel datasets. The findings from these inequality estimate suggest that efforts to improve self-management should focus on addressing socio-economic inequalities. The distribution of diabetes ill-health in South Africa, indicates how important it is for the South African government, through the country's health care system and other sectors, to improve access to health care and the adoption of healthy lifestyles across all socio-economic groups. Our findings from the catastrophic health expenditure inequalities calls for targeted policies that protect people with diabetes within low socio-economic groups from catastrophic health expenditure.

Statement 2: Financial difficulty amongst people with diabetes in South Africa is both an outcome of self-care and a barrier to some diabetes self-care practices

As indicated in the introductory chapter, diabetes is best managed when patients have access to health care for frequent check-ups to monitor the development of diabetes complications such as those discussed in Chapter 6. Diabetes is also best managed when patients adhere to recommended diabetes self-care practices such as healthy diets, limited alcohol consumption 
and physical activity (American Diabetes Association, 2017; IDF, 2017; WHO, 2016a). In instances where patients are required to pay OOP for health care, this could result in financial difficulty. Financial difficulty is also associated with non-adherence to various diabetes selfcare behaviours. This is reflected in previous research and findings from Chapter 2, 3, 5 and 6 of the dissertation. Chapter 2 finds that most of the direct costs associated with diabetes in Africa are borne by the patient. Chapter 3 shows that adherence to the self-care practice of health care check-up may result in catastrophic financial difficulties. In Chapter 5 and 6, we show that lack of finances may also lead to non-adherence to some self-care practices.

The literature review in Chapter 1 confirms the paucity of research on the economic impact of diabetes in South Africa. Thus, to address the second objective of this dissertation, we examined the costs associated with diabetes health care in South Africa. The introductory chapter provides an overview of the health care system in South Africa, highlighting how public sector health care is financed. Despite the modest health care fees in public hospitals, the results from Chapter 3 show that financial protection measures in South African public hospitals have only limited potential to protect people with diabetes from catastrophic health expenditure. Our results show that between $13 \%$ and $25 \%$ of people with diabetes visiting the two public hospitals suffered catastrophic health expenditure as a result of health care costs, whilst between $2 \%$ to $4 \%$ were impoverished as a result of health care costs (see Table 3-4, Chapter 3). A major contributing factors to these costs are the costs associated with transport when accessing health care facilities. The incidence of catastrophic health expenditure due to diabetes (Gwatidzo \& Williams, 2017; Okoronkwo et al., 2015) and the impoverishing nature of health care costs have previously been reported in other studies (Kwesiga, Zikusooka, \& Ataguba, 2015; Ngcamphalala \& Ataguba, 2018). This incidence of catastrophic health care expenditure is of concern given that diabetes is a life-long condition requiring patients to make multiple visits to health care facilities. Furthermore, people with diabetes do not only incur costs associated with accessing health care but must also contend with the costs associated with adhering to recommended self-care practices.

Financial difficulties are not only an outcome of diabetes self-care but they act as a barrier to some self-care practices too. The financial implications of diabetes management lead to some patients sacrificing elements of their recommended diabetes self-care practices such as 
adhering to a healthy diet and self-monitoring of blood glucose (Campbell et al., 2017; Patel, Piette, Resnicow, Kowalski-Dobson, \& Heisler, 2016). When faced with these financial barriers, patients resort to draining their savings or practicing cost reducing behavioural strategies such as omitting hospital check-ups, reusing disposable supplies or self-adjusting medication schedules (Xin Feng, Manns, \& Campbell, 2018). Consistent with other studies we find that financial difficulties are one of the reasons why people with diabetes do not adhere to some recommended diabetes self-care practices (Patel et al., 2016). Our findings from the qualitative study reported in Chapter 6, show that in South Africa, the cost of diabetes management limits the potential for adhering to recommended diabetes self-care practices such as a healthy diet. This finding is also supported by results from Chapter 5 , which show an association between the wealth index and some aspects of diabetes self-care such as dietary diversity. Consistent with other studies, we find that dominant aspects of care, which are limited by financial constraints, are diet and diabetes self-monitoring supplies (Campbell et al., 2017). In particular, patients in our qualitative study in Chapter 6, report on how difficult it is to adhere to recommended dietary practices due to the lack of money. Other patients reported that cost of glucose monitoring strips is a barrier to the self-monitoring of glucose levels. Although more frequently reported by participants from the public hospital in our study, some participants visiting the private sector also noted how the lack of affordability acted as a barrier to some self-care practices. Such practices may have adverse health outcomes via their impact on glycaemic levels (Campbell et al., 2017; Patel et al., 2016). This is supported by findings from Chapter 7, which show that compared to those of low socioeconomic status, those within the high socio-economic groups are less likely to report diabetes CVD comorbidity.

\section{Research and policy implications}

The estimation of catastrophic health care expenditure due to diabetes in our study made use of data collected from two diabetes clinics in Tshwane. It would be useful for future studies to make use of nationally representative datasets that includes people with diabetes that make use of both the private and the public sector. Furthermore, future research should aim to establish the incidence of catastrophic health expenditure amongst people with diabetes with complications versus those without. The findings from our study call for the elimination 
of user fees for people with diabetes accessing public hospitals and targeted interventions to reduce the transport costs associated with accessing health care facilities. Such targeted interventions will allow for a reduction in health care costs amongst people with diabetes, leaving them room to pay for the costs associated with other recommended self-care behaviours. Furthermore, the financial challenges patients face should be considered in the tailoring of individualised patient care. The development of programmes and initiatives that overcome financial barriers to care is crucial.

Statement 3: The economic burden of diabetes in Africa is huge. Diabetes has both direct and indirect costs, which impact on individuals, households, societies, health care systems and governments

Related to objective 1 of this dissertation, we find that the economic burden of diabetes is huge. As indicated in Chapter 2, the direct costs of diabetes range from I\$3.5 billion to I\$4.5 billion per annum. Despite this burden, our literature review in Chapter 2 shows that there is a paucity of studies on the economic burden of diabetes in Africa. This finding is consistent with evidence from a more recent literature review on the economic impact of diabetes in Africa published in 2019 (Mapa-Tassou, Katte, Maadjhou, \& Mbanya, 2019). The emergence of diabetes as a threat to the health of populations in Africa is burdensome given the vulnerable nature of the population, the low resource settings in most of the countries and the occurrence of NCDs such as diabetes alongside a communicable diseases burden (Mbanya, Motala, Sobngwi, Assah, \& Enoru, 2010). Furthermore, the presence of comorbidities increases the economic burden of diabetes as reflected in Chapter 1.

Forecasts show that the highest expected increases in diabetes are within the African region (IDF, 2019). The IDF forecasts a 48\% increase between the years 2019 and 2030 (IDF, 2019). In Africa, diabetes affects mainly those of working age, therefore the predicted increases in diabetes prevalence would place added pressure on economies that suffer already as a result of work absenteeism and reduced productivity. The burden of diabetes poses a catastrophic financial cost as most payments within the region are made OOP, by patients and families (Mapa-Tassou et al., 2019). This is revealed in findings from in a study included in our literature review (see Chapter 2) by Okoronkwo et al. (2015) and also findings from our study 
in Chapter 3. This financial burden could also drive non-poor households into poverty as we see in Chapter 3.

Assessing the economic impact of diabetes is useful for addressing various policy questions related to the consequences of diabetes. Whilst there is a wide array of methods used in the estimation of the economic burden of disease, the $\mathrm{COI}$ approach, which traditionally divides costs into direct, indirect and intangible costs, is the most commonly applied method. The literature review conducted in Chapter 2 suggests that the economic impact of diabetes is not only huge but it is distributed across various cost drivers which are identified as being both direct (for example consultation fees) or indirect costs (for example productivity losses due to work absenteeism) (see additional file 4 Chapter 2). Findings from our review in Chapter 2 show that drug costs took a significant portion of the total costs, whilst the results from our study in Chapter 3 show that transport costs took over $50 \%$ of total health care costs for South African patients visiting diabetes clinics at the public hospitals.

Although indirect costs are common amongst people with diabetes, our review in Chapter 2 shows that there is a paucity of studies that estimate the indirect costs of diabetes. Studies in our literature review that did calculate indirect costs showed that permanent disability accounted for the largest portion of indirect costs. Furthermore, comorbidities such as those discussed in Chapter 7 are a huge cause of increased diabetes related indirect costs. This finding is reflected in previous literature (Fu, Qiu, Radican, \& Wells, 2009; Leśniowska, Schubert, Wojna, Skrzekowska-Baran, \& Fedyna, 2014). People with diabetes and complications are likely to report more missed days from work and hospital visits. In Chapter 3, we also observe that diabetes has both indirect and direct economic implications on patients. When compared with direct costs due to diabetes and consistent with findings from our literature review presented in Chapter 2, our study in Chapter 3 finds that indirect costs are higher than the direct costs. Findings from the study in Chapter 6 also show the presence of indirect costs related to diabetes. The chapter revealed that having diabetes influenced productivity and employment. Patients reported job losses as a result of not being able to fulfil their duties at work. 
Our literature review presented in Chapter 2 showed that the costs associated with diabetes impact on individuals, households, societies, health care systems and governments. The studies included in the review showed that most of the health care costs associated with diabetes are borne by the patients. The costs patients incur at the point of health care use, are a function of the country's health care system. Chapter 1 introduces the structure of the South African health care system, highlighting the differences between the public and the private sector. The country's health care system is described as two tiered, consisting of a large public sector and a much smaller private sector that serves $16 \%$ of the population. The private sector is often described as world class whilst the public sector is faced by many challenges such as being poorly resourced. The private sector is available to individuals who can afford health insurance or non-insured individuals who can afford the private sector fees. On the other hand, in the public sector, primary health care is accessed for free and user fees within the hospitals are charged according to a UPFS. It is within these challenges that the public health care sector has to carry the responsibility of diabetes management for many people with diabetes.

Two South African studies included in our literature review in Chapter 2 reveal a high cost burden for hospital admissions (Ncube-Zulu \& Danckwerts, 2014; Pepper, Burch, Levitt, \& Cleary, 2007). A more recent study by Erzse et al. (2019) also shows that public sector costs due to diagnosed type 2 diabetes were R2.7 billion (USD 198 million) in 2018 (Erzse et al., 2019). These high costs are a cause for concern as they occur in public hospitals that serve the majority of the population as discussed in Chapter 1 of this dissertation. Given the predicted rise in diabetes, these costs have the potential to cripple an already strained public health sector.

\section{Research and policy implications}

As previously discussed, there is a paucity of studies on the economic impact of diabetes in African countries, in particular, national estimates of the economic impact of diabetes in African countries as well as studies that estimate the indirect costs of diabetes. Future research on the economic impact of diabetes should, in addition to using nationally representative datasets, also estimate the indirect costs associated with diabetes. 
Furthermore, since our study on South Africa focused only on people with diabetes visiting public sector hospitals, future studies should also estimate economic impact of diabetes amongst patients visiting private health care facilities. The burden of diabetes described in this study reflect an urgent need for policies that improve financial protection amongst people with diabetes visiting public sector hospitals.

Statement 4: Evidence in South Africa suggests that the factors that influence health care behaviour are varied and originate from multiple levels. The promotion of each behaviour requires separate attention

Chapter 1 of this dissertation introduces the role of self-care in the management of diabetes. Diabetes management is crucial to improve glycaemic levels, reducing the morbidity, mortality and economic burden associated with diabetes. Despite the importance of diabetes self-care, South African studies have documented the poor levels of adherence to diabetes self-care amongst patients and the poor glycaemic control (Nthangeni et al., 2002; Stokes et al., 2017). Such poor glycaemic control is linked to the development of diabetic complications (American Diabetes Association, 2017). From Chapter 1 we note that people with diabetes have an increased risk of acute complications such as diabetic commas and chronic illnesses such as heart disease (Emerging Risk Factors Collaboration, 2010; IDF, 2001, 2017). It is reported that people with diabetes are twice as likely to develop CVDs when compared to people without diabetes. As a result, they are at an increased risk of experiencing heart attack or strokes. This is seen in Chapter 7 where we find that over $73 \%$ of our diabetic sample reported having at least one additional CVD. The prevention or delay of such complications in people with diabetes involves a complex mix of diabetes management practices. In addition to the financial difficulties already discussed, there are a number of other factors that also influence these health care behaviours.

To address objective 4, which sort to determine the inequalities in diabetes self-care practices and the determinants of diabetes self-care practices in South Africa, we applied both quantitative and qualitative methods. From the quantitative study, we restricted ourselves to the analysis of six behaviours, dietary diversity, medication adherence, physical activity, selfmonitoring of blood glucose, avoiding smoking and limited alcohol consumption. After 
applying a common set of explanatory variables in Chapter 5, we find that the factors associated with each self-care practice are different. Even in cases where self-care behaviours have some statistically significant variables in common, the size of the effect differed. For example, we find that those aged 60 years and older had increased odds of adhering to diabetic medication and decreased odds of adhering to self-monitoring of blood glucose levels when compared to those within the 21 - 40 year age group. Also, whilst having tertiary education is associated with increased odds of adhering to exercise, having a tertiary education is not associated with the rest of the self-care practices in our study.

The determinants of self-care practices are not only influenced by socio-demographic characteristics as reflected in Chapter 5. Using qualitative data and the TTI, we show the various factors emanating from the intra-personal, inter-personal and environmental streams that either inhibit of enable diabetes self-care practices. Our study shows that although selfcare is mostly the patient's individual responsibility, committing to and adhering to self-care practices cannot be separated from the larger context of social relations and the environment in which people with diabetes must live. Our study in Chapter 6 identified intra-personal barriers and enablers to care, inter-personal barriers and enablers to care as well as environmental barriers and enablers to diabetes self-care. For example, at the inter-personal levels, we find that interactions with family and friends can act as both a barrier and an enabler of self-care.

In the qualitative study in Chapter 6, we interviewed patients visiting both the private and public health care diabetes clinics. Similarities and differences are picked up amongst the diabetes self-care experiences of patients visiting the two health care sectors. Whilst the enablers and inhibitors of diabetes self-care emanating from the intra-personal stream are common across all patients visiting both the public and private diabetes clinics, for the interpersonal and environmental streams some differences are evident. These differences are a result of the inequalities that exist between the South African public health care system and the private health care system as discussed in Chapter 1. For example, although costly, access to health insurance by patients attending the private clinic means patients have access to insulin pumps, which helped ensure medication adherence. On the other hand, patients 
attending the public clinics make use of oral diabetic medication and insulin injections or pens.

\section{Research and policy implications}

Future quantitative research should make use of a nationally representative dataset to estimate the inequalities and determinants of diabetes self-care for patients attending both the private and public health care facilities. Given the rich cultural diversity of South Africa, future qualitative studies on diabetes management experiences should include explicit queries or references to culture within the environmental stream. Given that the determinants of self-care and the magnitude of their effect is not consistent across the selfcare behaviours, interventions that aim to improve diabetes self-care practise should take into consideration the health behaviour to be addressed and the context in which the behaviour occurs. Given that multi-level factors influence the occurrence of diabetes selfcare, programmes that promote self-care need to be comprehensive and target all levels of influence.

\section{Statement 5: Diabetes is a multifaceted multivariable disease linked with other diseases.} This emphasises the need for a holistic approach to its management.

This dissertation demonstrates that diabetes has many aspects to it. Patients encounter a multi-faceted multivariable disease not only regarding its pathological sub-types, which are discussed in Chapter 1, or its management, but also regarding its association with various other chronic illnesses and acute complications. Chapter 1 of the dissertation introduces the burden of diabetes complications. From Chapter 1, we note that people with diabetes have an increased risk of both acute complications such as diabetic commas, and chronic illnesses such as heart disease. People with diabetes are twice as likely to develop CVD when compared to people without diabetes. As a result, they are at an increased risk of experiencing heart attack or strokes. This is seen in Chapter 7 where we find that over $73 \%$ of our diabetic sample reported having at least one additional CVD. The presence of complications among people with diabetes is associated with an increased burden of disease, which is often demonstrated 
by its huge economic impact. Preventing the development of these complications is an important step to reducing their burden.

The results from the regression in Chapter 7, suggest that after applying a common set of variables, the statistical significance of the effect of each determinant on diabetes or diabetes CVD comorbidity is different. Even cases in which variables are associated with both diabetes and diabetes CVD comorbidity, the magnitude of the effect differed. We find for example that older age is associated with both the presence of diabetes and the presence of diabetes CVD comorbidity. The magnitude of the coefficients is slightly larger for diabetes CVD when compared to diabetes only. This indicates a slightly higher likelihood of association. Other differences are for example that being Indian is associated with having diabetes but not associated with diabetes CVD comorbidity, high income is associated with diabetes CVD comorbidity but not with diabetes. These findings give insight into the factors associated with having diabetes only and having diabetes and CVD comorbidity. The results can aid decision making for improved diabetes outcomes.

\section{Research and policy implications}

The high prevalence of diabetes and CVD comorbidities evident in our study calls for interventions that work towards reducing the development of diabetes CVD comorbidity and where diabetes CVD comorbidity is present, a holistic approach in diabetes management is needed. As indicated in our regression results in Chapter 7, social aspects are also important for the management of diabetes and diabetes CVD comorbidity. There are however gaps in our understanding of the factors that influence diabetes CVD comorbidity. Other than the CVDs covered in our study (stroke high blood pressure and heart disease), people with diabetes have an increased risk of developing not only other CVDs (such as heart attack) but also other chronic illnesses and acute complications. Thus, future studies should aim to include other comorbidity conditions when assessing the factors associated with having diabetes and other illnesses. More studies still need to be done in order to concretely establish the determinants of diabetes comorbidity. 


\subsection{Concluding remarks}

Aside, from its impact on (mental) health, well-being and activities of daily living, diabetes places a huge economic burden on patients and their families. Given the importance of selfcare behaviours, such as frequent hospital check-ups and adherence to recommended lifestyle practices in influencing diabetes outcomes, policies that support such diabetes selfcare management practices are becoming increasingly crucial. This dissertation has revealed the difficulties experienced by patients when accessing health care and when trying to adhere to diabetes self-care practices such as a healthy diet. Given that people with diabetes have to make multiple visits to health care facilities for frequent health checks, the provision of affordable health care is central to protecting patients from catastrophic expenditure as a result of diabetes health care. This dissertation has also demonstrated the prevalence of nonadherence to recommended diabetes care. Cost-related non-adherence to self-care practices such as healthy eating or self-monitoring of blood glucose may contribute to adverse diabetes outcomes. Whilst financial barriers are a common inhibitor of self-care, our dissertation highlights the existence of multi-level determinants of self-care. Policies directed at promoting diabetes self-care should carefully consider the intra-personal, inter-personal and environmental influences of self-care. The dissertation also demonstrated the inequalities in diabetes prevalence, economic burden and self-care practices. This is why we posit for policies that address the huge income inequalities in South Africa. 


\section{References}

Abdulganiyu, G., \& Fola, T. (2014). What is the cost of illness of type II Diabetes Mellitus in a Developing Economy. Int J Pharm Pharm Sci, 6, 927-931.

Addo, J., Agyemang, C., Aikins, A. d.-G., Beune, E., Schulze, M. B., Danquah, I., . . KlipsteinGrobusch, K. (2017). Association between socioeconomic position and the prevalence of type 2 diabetes in Ghanaians in different geographic locations: the RODAM study. J Epidemiol Community Health, jech-2016-208322.

Adegbola, S. A., Marincowitz, G. J., Govender, I., \& Ogunbanjo, G. A. (2016). Assessment of self-reported adherence among patients with type 2 diabetes in Matlala District Hospital, Limpopo Province. African journal of primary health care \& family medicine, 8(1).

Adeniyi, O. V., Yogeswaran, P., Wright, G., \& Longo-Mbenza, B. (2015). Diabetic patients' perspectives on the challenges of glycaemic control. African journal of primary health care \& family medicine, $7(1), 1-8$.

Adu, M. D., Malabu, U. H., Malau-Aduli, A. E., \& Malau-Aduli, B. S. (2019). Enablers and barriers to effective diabetes self-management: A multi-national investigation. PloS one, 14(6), e0217771.

African National Congress. 3rd National General Council. (2010). Road to Health. Additional Discussion Documents: Section 1, National Health Insurance: 3 - 89. .

Agardh, E. E., Ahlbom, A., Andersson, T., Efendic, S., Grill, V., Hallqvist, J., \& Östenson, C.-G. (2004). Explanations of socioeconomic differences in excess risk of type 2 diabetes in Swedish men and women. Diabetes Care, 27(3), 716-721.

Agardh, E., Allebeck, P., Hallqvist, J., Moradi, T., \& Sidorchuk, A. (2011). Type 2 diabetes incidence and socio-economic position: a systematic review and meta-analysis. International journal of epidemiology, 40(3), 804-818.

Agborsangaya, C. B., Lau, D., Lahtinen, M., Cooke, T., \& Johnson, J. A. (2012). Multimorbidity prevalence and patterns across socioeconomic determinants: a cross-sectional survey. BMC public health, 12(1), 1.

Alaba, O., \& Chola, L. (2013). The social determinants of multimorbidity in South Africa. International journal for equity in health, 12(1), 1.

Alaba, O., \& Chola, L. (2014). Socioeconomic inequalities in adult obesity prevalence in South Africa: a decomposition analysis. International journal of environmental research and public health, 11(3), 3387-3406.

Alouki, K., Delisle, H., Besançon, S., Baldé, N., Sidibé-Traoré, A., Drabo, J., . . Halimi, S. (2015). Simple calculator to estimate the medical cost of diabetes in sub-Saharan Africa. World J Diabetes, 6(16), 1312.

American Diabetes Association. (2017). Standards of medical care in diabetes-2017. Diabetes Care., 40 (Suppl. 1), 1-142. 
American Diabetes Association. (2015). 2. Classification and diagnosis of diabetes. Diabetes care, 38(Supplement 1), S8-S16.

American Diabetes Association. (2018). Economic Costs of Diabetes in the US in 2017. Diabetes Care, 41(5), 917-928.

Amod, A. (2012). The 2012 SEMDSA guideline for the management of type 2 diabetes. Journal of Endocrinology, Metabolism and Diabetes in South Africa, 17(1), 61-62.

Aregbeshola, B. S., \& Khan, S. M. (2018). Out-of-pocket payments, catastrophic health expenditure and poverty among households in Nigeria 2010. International journal of health policy and management, 7(9), 798.

Ataguba, J. E., \& McIntyre, D. (2018). The incidence of health financing in South Africa: findings from a recent data set. Health Economics, Policy and Law, 13(1), 68-91.

Ataguba, J. E., Akazili, J., \& Mclntyre, D. (2011). Socioeconomic-related health inequality in South Africa: evidence from General Household Surveys. International journal for equity in health, 10(1), 48.

Ataguba, J. E., Ojo, K. O., \& Ichoku, H. E. (2016). Explaining socio-economic inequalities in immunization coverage in Nigeria. Health policy and planning, 31(9), 1212-1224.

Ataguba, J. E.-O. (2012). Reassessing catastrophic health-care payments with a Nigerian case study. Health Economics, Policy and Law, 7(3), 309-326.

Ataguba, J. E.-O. (2013). Inequalities in multimorbidity in South Africa. International journal for equity in health, 12(1), 64.

Ataguba, J. E.-O., Day, C., \& McIntyre, D. (2015). Explaining the role of the social determinants of health on health inequality in South Africa. Global health action, 8(1), 28865.

Ataguba, J., \& Akazili, J. (2010). Health care financing in South Africa: moving towards universal coverage. Continuing Medical Education, 28(2).

Ataguba, J., \& Alaba, O. (2012). Explaining health inequalities in South Africa: a political economy perspective. Development Southern Africa, 29(5), 756-764.

Aune, D., Norat, T., Leitzmann, M., Tonstad, S., \& Vatten, L. J. (2015). Physical activity and the risk of type 2 diabetes: a systematic review and dose-response meta-analysis. In: Springer.

Babikir, A., Satty, A., \& Mwambi, H. (2018). Determinants of out-of-pocket health expenditure and their welfare implications in a South African context. Journal of Economic and Financial Sciences, 11(1), 7.

Bagonza, J., Rutebemberwa, E., \& Bazeyo, W. (2015). Adherence to anti diabetic medication among patients with diabetes in eastern Uganda; a cross sectional study. BMC health services research, 15(1), 168.

Baig, A. A., Benitez, A., Quinn, M. T., \& Burnet, D. L. (2015). Family interventions to improve diabetes outcomes for adults. Annals of the New York Academy of Sciences, 1353(1), 89. 
Balanda, K. P., Buckley, C. M., Barron, S. J., Fahy, L. E., Madden, J. M., Harrington, J. M., . . . Kearney, P. M. (2013). Prevalence of diabetes in the Republic of Ireland: results from the National Health Survey (SLAN) 2007. PloS one, 8(10), e78406.

Baliunas, D. O., Taylor, B. J., Irving, H., Roerecke, M., Patra, J., Mohapatra, S., \& Rehm, J. (2009). Alcohol as a risk factor for type 2 diabetes. Diabetes care, 32(11), 2123-2132.

Barnett, K., Mercer, S. W., Norbury, M., Watt, G., Wyke, S., \& Guthrie, B. (2012). Epidemiology of multimorbidity and implications for health care, research, and medical education: a crosssectional study. The Lancet, 380(9836), 37-43.

Basu, S., Shankar, V., \& Yudkin, J. S. (2016). Comparative effectiveness and cost-effectiveness of treat-to-target versus benefit-based tailored treatment of type 2 diabetes in low-income and middle-income countries: a modelling analysis. The Lancet Diabetes \& Endocrinology, 4(11), 922-932.

Bazzano A. L. (2005). Dietary intake of fruit and vegetables and risk of diabetes mellitus and cardiovascular disease. Background paper for the joint FAO/WHO workshop on fruit and vegetable for health. Geneva: WHO

Beaglehole, R., Bonita, R., Horton, R., Adams, C., Alleyne, G., Asaria, P., .. Casswell, S. (2011). Priority actions for the non-communicable disease crisis. The Lancet, 377(9775), 1438-1447.

Becker, C. (2014). Cost-of-illness studies of atrial fibrillation: methodological considerations. Expert review of pharmacoeconomics \& outcomes research, 14(5), 661-684.

Beenackers, M. A., Kamphuis, C. B., Giskes, K., Brug, J., Kunst, A. E., Burdorf, A., \& Van Lenthe, F. J. (2012). Socioeconomic inequalities in occupational, leisure-time, and transport related physical activity among European adults: a systematic review. International journal of behavioral nutrition and physical activity, 9(1), 116.

Bennich, B. B., Røder, M. E., Overgaard, D., Egerod, I., Munch, L., Knop, F. K., . . . Konradsen, H. (2017). Supportive and non-supportive interactions in families with a type 2 diabetes patient: an integrative review. Diabetology \& metabolic syndrome, 9(1), 57.

Beverly, E. A., Worley, M., Prokopakis, K., \& Ivanov, N. (2016). Patient-physician communication and diabetes self-care. Journal of Clinical Outcomes Management, 23(11), 509-518.

Bhana, A., Rathod, S. D., Selohilwe, O., Kathree, T., \& Petersen, I. (2017). Characteristics and correlates of alcohol consumption among adult chronic care patients in North West Province, South Africa. South African Medical Journal, 107(7), 636-642.

Biswas, T., Islam, M. S., Linton, N., \& Rawal, L. B. (2016). Socio-economic inequality of chronic non-communicable diseases in Bangladesh. PloS one, 11(11), e0167140.

Bloom, B. S., Bruno, D. J., Maman, D. Y., \& Jayadevappa, R. (2001). Usefulness of US cost-ofillness studies in healthcare decision making. Pharmacoeconomics, 19(2), 207-213. 
Bogoev, J., Dessus, S. C., Hanusch, M., Kwaramba, M., \& Whyte, S. (2018). Southern African customs union - Macro poverty outlook: country by country analysis and projection for the developing world. Washington, D.C. World Bank Group.

Bommer, C., Sagalova, V., Heesemann, E., Manne-Goehler, J., Atun, R., Bärnighausen, T., . . . Vollmer, S. (2018). Global economic burden of diabetes in adults: projections from 2015 to 2030. Diabetes Care, 41(5), 963-970.

Bonger, Z., Shiferaw, S., \& Tariku, E. Z. (2018). Adherence to diabetic self-care practices and its associated factors among patients with type 2 diabetes in Addis Ababa, ethiopia. Patient preference and adherence, 12, 963.

Booysen, B. L., \& Schlemmer, A. C. (2015). Reasons for diabetes patients attending Bishop Lavis Community Health Centre being non-adherent to diabetes care. South African Family Practice, 57(3), 166-171.

Booysen, F., Van Der Berg, S., Burger, R., Von Maltitz, M., \& Du Rand, G. (2008). Using an asset index to assess trends in poverty in seven Sub-Saharan African countries. World Development, 36(6), 1113-1130.

Borg, V., \& Kristensen, T. S. (2000). Social class and self-rated health: can the gradient be explained by differences in lifestyle or work environment? Social science \& medicine, 51(7), 1019-1030.

Boutayeb, W., Lamlili, M. E., Boutayeb, A., \& Boutayeb, S. (2013). Estimation of direct and indirect cost of diabetes in Morocco. Journal of Biomedical Science and Engineering 3(7): 732738.

Bovet, P., Shamlaye, C., Gabriel, A., Riesen, W., \& Paccaud, F. (2006). Prevalence of cardiovascular risk factors in a middle-income country and estimated cost of a treatment strategy. BMC public health, 6(1), 9.

Bradshaw, D., Groenewald, P., Laubscher, R., Nannan, N., Nojilana, B., Norman, R., . . . Timæus, I. M. (2003). Initial burden of disease estimates for South Africa, 2000. South African Medical Journal, 93(9), 682-688.

Buigut, S., Ettarh, R., \& Amendah, D. D. (2015). Catastrophic health expenditure and its determinants in Kenya slum communities. International journal for equity in health, 14(1), 46.

Burger, R., \& Christian, C. (2018). Access to health care in post-apartheid South Africa: Availability, affordability, acceptability. Health Economics, Policy and Law, 1-13.

Campbell, D. J., Manns, B. J., Hemmelgarn, B. R., Sanmartin, C., Edwards, A., \& King-Shier, K. (2017). Understanding financial barriers to care in patients with diabetes: an exploratory qualitative study. The Diabetes Educator, 43(1), 78-86.

Carlsson, S., Hammar, N., Grill, V., \& Kaprio, J. (2003). Alcohol consumption and the incidence of type 2 diabetes: a 20-year follow-up of the Finnish twin cohort study. Diabetes care, 26(10), 2785-2790. 
Carter, P., Gray, L. J., Troughton, J., Khunti, K., \& Davies, M. J. (2010). Fruit and vegetable intake and incidence of type 2 diabetes mellitus: systematic review and meta-analysis. BMJ, 341, c4229.

Castillo-Riquelme, M., Mclntyre, D., \& Barnes, K. (2008). Household burden of malaria in South Africa and Mozambique: is there a catastrophic impact? Tropical Medicine \& International Health, 13(1), 108-122.

Cavanagh, P., Attinger, C., Abbas, Z., Bal, A., Rojas, N., \& Xu, Z. R. (2012). Cost of treating diabetic foot ulcers in five different countries. Diabetes/metabolism research and reviews, 28(S1), 107-111.

Chlebowy, D. O., Hood, S., \& LaJoie, A. S. (2010). Facilitators and barriers to self-management of type 2 diabetes among urban African American adults. The Diabetes Educator, 36(6), 897905.

Chuma, J., \& Maina, T. (2012). Catastrophic health care spending and impoverishment in Kenya. BMC health services research, 12(1), 413.

City of Tshwane. (2016). City of Tshwane, 2016/21 IDP. Retrieved from: http://www.tshwane.gov.za/Documents/Tabling\%20of\%20the\%20City\%20of\%20Tshwane\% 20Integrated\%20Development\%20Plan\%20for\%202016\%202021.pdf

Cleary, S. M., Birch, S., Moshabela, M., \& Schneider, H. (2012). Unequal access to ART: exploratory results from rural and urban case studies of ART use. Sexually Transmitted Infections, 88(2), 141-146.

Cleary, S., Birch, S., Chimbindi, N., Silal, S., \& McIntyre, D. (2013). Investigating the affordability of key health services in South Africa. Social science \& medicine, 80, 37-46.

Clement, S. (1995). Diabetes self-management education. Diabetes Care, 18(8), 1204-1214.

Conigrave, K. M., Hu, B. F., Camargo, C. A., Stampfer, M. J., Willett, W. C., \& Rimm, E. B. (2001). A prospective study of drinking patterns in relation to risk of type 2 diabetes among men. Diabetes, 50(10), 2390-2395.

Cooper, A. J., Forouhi, N. G., Ye, Z., Buijsse, B., Arriola, L., Balkau, B., . . Büchner, F. L. (2012). Fruit and vegetable intake and type 2 diabetes: EPIC-InterAct prospective study and metaanalysis. European journal of clinical nutrition, 66(10), 1082.

Costa, N., Derumeaux, H., Rapp, T., Garnault, V., Ferlicoq, L., Gillette, S., . . Grand, A. (2012). Methodological considerations in cost of illness studies on Alzheimer disease. Health economics review, 2(1), 18.

Council for Medical Schemes. (2017). Annual Report 2016-2017. Retrieved from https://www.medicalschemes.com/Publications.aspx

Dalstra, J., Kunst, A., Borrell, C., Breeze, E., Cambois, E., Costa, G., . . Mackenbach, J. (2005). Socioeconomic differences in the prevalence of common chronic diseases: an overview of 
eight European countries. International journal of epidemiology, 34(2), 316-326. doi:10.1093/ije/dyh386

Daniels, A., Biesma, R., Otten, J., Levitt, N. S., Steyn, K., Martell, R., \& Dick, J. (2000). Ambivalence of primary health care professionals towards the South African guidelines for hypertension and diabetes. S Afr Med J, 90(12), 1206-1211.

Danmusa, U. M., Terhile, I., Nasir, I. A., Ahmad, A. A., \& Muhammad, H. Y. (2016). Prevalence and healthcare costs associated with the management of diabetic foot ulcer in patients attending Ahmadu Bello University Teaching Hospital, Nigeria. International journal of health sciences, 10(2), 219.

De Silva, A. P., De Silva, S. H. P., Haniffa, R., Liyanage, I. K., Jayasinghe, S., Katulanda, P., . . . Rajapaksa, L. C. (2018). Inequalities in the prevalence of diabetes mellitus and its risk factors in Sri Lanka: a lower middle-income country. International journal for equity in health, $17(1)$, 45.

Deshpande, A. D., Harris-Hayes, M., \& Schootman, M. (2008). Epidemiology of diabetes and diabetes-related complications. Physical therapy, 88(11), 1254-1264.

Diabetes Control and Complications Trial Research Group. (1993). The effect of intensive treatment of diabetes on the development and progression of long-term complications in insulin-dependent diabetes mellitus. New England journal of medicine, 329(14), 977-986.

Doherty, E., Walsh, B., \& O'Neill, C. (2014). Decomposing socioeconomic inequality in child vaccination: results from Ireland. Vaccine, 32(27), 3438-3444.

Drummond, M., Sculpher, M., Torrance, G., O’Brien, B., \& Stoddart, G. (2005). Methods for the economic evaluation of health care programmes (3rd ed.): Oxford University Press.

Du, H., Li, L., Bennett, D., Guo, Y., Turnbull, I., Yang, L., . . . Chen, J. (2017). Fresh fruit consumption in relation to incident diabetes and diabetic vascular complications: a 7-y prospective study of 0.5 million Chinese adults. PLoS medicine, 14(4), e1002279.

Dybicz, S. B., Thompson, S., Molotsky, S., \& Stuart, B. (2011). Prevalence of diabetes and the burden of comorbid conditions among elderly nursing home residents. The American journal of geriatric pharmacotherapy, 9(4), 212-223.

Dyer, S. J., Sherwood, K., McIntyre, D., \& Ataguba, J. E. (2013). Catastrophic payment for assisted reproduction techniques with conventional ovarian stimulation in the public health sector of South Africa: frequency and coping strategies. Human Reproduction, 28(10), 27552764.

Earnshaw, V. A., Bogart, L. M., Courtney, I., Zanoni, H., Bangsberg, D. R., Orrell, C., . . Katz, I. T. (2018). Exploring treatment needs and expectations for people living with HIV in South Africa: a qualitative study. AIDS and Behavior, 22(8), 2543-2552.

Ebrahim, Z., De Villiers, A., \& Ahmed, T. (2014). Factors influencing adherence to dietary guidelines: a qualitative study on the experiences of patients with type 2 diabetes attending 
a clinic in Cape Town. Journal of Endocrinology, Metabolism and Diabetes of South Africa, 19(2), 76-84.

Efron, B. (1987). Better bootstrap confidence intervals. Journal of the American Statistical Association, 82(397), 171-185.

Efron, B., \& Tibshirani, R. (1986). Bootstrap methods for standard errors, confidence intervals, and other measures of statistical accuracy. Statistical science, 54-75.

Egbujie, B. A., Igumbor, E. U., \& Puoane, T. (2016). A cross-sectional study of socioeconomic status and cardiovascular disease risk among participants in the Prospective Urban Rural Epidemiological (PURE) Study. South African Medical Journal, 106(9), 900-906.

Elgar, F. J. (2010). Income inequality, trust, and population health in 33 countries. American Journal of Public Health, 100(11), 2311-2315.

Elo, S., \& Kyngäs, H. (2008). The qualitative content analysis process. Journal of advanced nursing, 62(1), 107-115.

Elo, S., Kääriäinen, M., Kanste, O., Pölkki, T., Utriainen, K., \& Kyngäs, H. (2014). Qualitative content analysis: A focus on trustworthiness. SAGE open, 4(1), 2158244014522633.

Elrayah-Eliadarous, H., Yassin, K., Eltom, M., Abdelrahman, S., Wahlström, R., \& Östenson, C.G. (2010). Direct costs for care and glycaemic control in patients with type 2 diabetes in Sudan. Experimental and clinical endocrinology \& diabetes, 118(04), 220-225.

Emerging Risk Factors Collaboration. (2010). Diabetes mellitus, fasting blood glucose concentration, and risk of vascular disease: a collaborative meta-analysis of 102 prospective studies. The lancet, 375(9733), 2215-2222.

Emerging Risk Factors Collaboration. (2011). Diabetes mellitus, fasting glucose, and risk of cause-specific death. New England journal of medicine, 364(9), 829-841.

Engler, P. A., Ramsey, S. E., \& Smith, R. J. (2013). Alcohol use of diabetes patients: the need for assessment and intervention. Acta diabetologica, 50(2), 93-99.

Enwere, O., Salako, B., \& Falade, C. (2006). Prescription and cost consideration at a diabetic clinic in Ibadan, Nigeria: A report. Annals of Ibadan postgraduate medicine, 4(2).

Erasmus, R., Blanco, E. B., Okesina, A., Gqweta, Z., \& Matsha, T. (1999). Assessment of glycaemic control in stable type 2 black South African diabetics attending a peri-urban clinic. Postgraduate medical journal, 75(888), 603-606.

Erreygers, G. (2009). Correcting the concentration index. Journal of health economics, 28(2), 504-515.

Erreygers, G., \& Kessels, R. (2013). Regression-based decompositions of rank-dependent indicators of socioeconomic inequality of health. In Rosa Dias P \& O'Donnell O (Eds.), Health and Inequality (Research on Economic Inequality, Volume 21, Chapter 9) (pp. 227-259). London: Emerald Group Publishing Limited. 
Erzse, A., Stacey, N., Chola, L., Tugendhaft, A., Freeman, M., \& Hofman, K. (2019). The direct medical cost of type 2 diabetes mellitus in South Africa: a cost of illness study. Global health action, 12(1), 1636611.

Ettaro, L., Zhang, P., \& Engelgau, M. M. (2004). Cost-of-illness studies in diabetes mellitus. Pharmacoeconomics, 22(3), 149-164.

Fadare, J., Olamoyegun, M., \& Gbadegesin, B. (2015). Medication adherence and direct treatment cost among diabetes patients attending a tertiary healthcare facility in Ogbomosho, Nigeria. Malawi Medical Journal, 27(2), 65-70.

Feleke, Y., \& Enquselassie, F. (2007). Cost of hospitalization of diabetic patients admitted at Tikur Anbessa Specialized Hospital, Addis Ababa, Ethiopia. Ethiop Med J, 45(3), 275-282.

Firpo, S., Fortin, N. M., \& Lemieux, T. (2009). Unconditional quantile regressions. Econometrica, 77(3), 953-973.

Flay, B. R., Snyder, F., \& Petraitis, J. (2009). The theory of triadic influence. Emerging theories in health promotion practice and research, 2, 451-510.

Folb, N., Timmerman, V., Levitt, N. S., Steyn, K., Bachmann, M. O., Lund, C., . . Z Zwarenstein, M. (2015). Multimorbidity, control and treatment of non-communicable diseases among primary healthcare attenders in the Western Cape, South Africa. SAMJ: South African Medical Journal, 105(8), 642-647.

Fortin, M., Dubois, M.-F., Hudon, C., Soubhi, H., \& Almirall, J. (2007). Multimorbidity and quality of life: a closer look. Health and quality of life outcomes, 5(1), 1.

Freeman, J., \& Loewe, R. (2000). Barriers to communication about diabetes mellitus. Journal of Family Practice, 49(6), 507-507.

Fu, A. Z., Qiu, Y., Radican, L., \& Wells, B. J. (2009). Health care and productivity costs associated with diabetic patients with macrovascular comorbid conditions. Diabetes Care, 32(12), 21872192.

Funnell, M. M., \& Anderson, R. M. (2000). The problem with compliance in diabetes. JaMa, 284(13), 1709-1709.

GBD 2013 Mortality and Causes of Death Collaborators. (2015). Global, regional, and national age-sex specific all-cause and cause-specific mortality for 240 causes of death, 1990-2013: a systematic analysis for the global burden of disease study 2013. The lancet, 385(9963), 117171.

GBD 2015 Mortality and Causes of Death Collaborators. (2016). Global, regional, and national life expectancy, all-cause mortality, and cause-specific mortality for 249 causes of death, 1980-2015: a systematic analysis for the Global Burden of Disease Study 2015. The lancet, 388(10053), 1459-1544.

Gill, G., Mbanya, J.-C., Ramaiya, K., \& Tesfaye, S. (2009). A sub-Saharan African perspective of diabetes. Diabetologia, 52(1), 8. 
Gill, G., Rolfe, M., MacFarlane, I., \& Huddle, K. (1996). Smoking habits of black South African patients with diabetes mellitus. Diabetic Medicine, 13(11), 996-999.

George, J. A. (2011). Should haemoglobin A1c be used for the diagnosis of diabetes mellitus in South Africa? Journal of Endocrinology, Metabolism and Diabetes of South Africa, 16(3), $122-127$

Gonzalo-Almorox, E., \& Urbanos-Garrido, R. M. (2016). Decomposing socio-economic inequalities in leisure-time physical inactivity: the case of Spanish children. International journal for equity in health, 15(1), 106.

Goudge, J., Gilson, L., Russell, S., Gumede, T., \& Mills, A. (2009). The household costs of health care in rural South Africa with free public primary care and hospital exemptions for the poor. Tropical Medicine \& International Health, 14(4), 458-467.

Govender, R. D., Gathiram, P., \& Panajatovic, M. (2017). Poor control and management of type 2 diabetes mellitus at an under-resourced South African Hospital: is it a case of clinical inertia? South African Family Practice, 59(5), 154-159.

Greene, K. M., Murphy, S. T., \& Rossheim, M. E. (2018). Context and culture: Reasons young adults drink and drive in rural America. Accident Analysis \& Prevention, 121, 194-201.

Guan, W. (2003). From the help desk: bootstrapped standard errors. The Stata journal, 3(1), 71-80.

Guariguata, L., Whiting, D. R., Hambleton, I., Beagley, J., Linnenkamp, U., \& Shaw, J. E. (2014). Global estimates of diabetes prevalence for 2013 and projections for 2035. Diabetes research and clinical practice, 103(2), 137-149.

Guerci, B., Drouin, P., Grange, V., Bougneres, P., Fontaine, P., Kerlan, V., . . Charbonnel, B. (2003). Self-monitoring of blood glucose significantly improves metabolic control in patients with type 2 diabetes mellitus: the Auto-Surveillance Intervention Active (ASIA) study. Diabetes \& metabolism, 29(6), 587-594.

Guh, D. P., Zhang, W., Bansback, N., Amarsi, Z., Birmingham, C. L., \& Anis, A. H. (2009). The incidence of co-morbidities related to obesity and overweight: a systematic review and metaanalysis. BMC public health, 9(1), 88.

Gulliford, M., Sedgwick, J., \& Pearce, A. (2003). Cigarette smoking, health status, socioeconomic status and access to health care in diabetes mellitus: a cross-sectional survey. BMC health services research, 3(1), 4.

Gurmu, Y., Gela, D., \& Aga, F. (2018). Factors associated with self-care practice among adult diabetes patients in West Shoa Zone, Oromia Regional State, Ethiopia. BMC health services research, 18(1), 732.

Gwatidzo, S. D., \& Williams, J. S. (2017). Diabetes mellitus medication use and catastrophic healthcare expenditure among adults aged 50+ years in China and India: results from the WHO study on global AGEing and adult health (SAGE). BMC geriatrics, 17(1), 14. 
Ha, J. F., \& Longnecker, N. (2010). Doctor-patient communication: a review. Ochsner Journal, 10(1), 38-43.

Hall, V., Thomsen, R. W., Henriksen, O., \& Lohse, N. (2011). Diabetes in Sub Saharan Africa 1999-2011: epidemiology and public health implications. A systematic review. BMC public health, 11(1), 564.

Harris, B., Goudge, J., Ataguba, J. E., McIntyre, D., Nxumalo, N., Jikwana, S., \& Chersich, M. (2011). Inequities in access to health care in South Africa. Journal of public health policy, 32(1), S102-S123.

Harris, M. I., Cowie, C. C., \& Howie, L. J. (1993). Self-monitoring of blood glucose by adults with diabetes in the United States population. Diabetes Care, 16(8), 1116-1123.

Hatløy, A., Torheim, L., \& Oshaug, A. (1998). Food variety-a good indicator of nutritional adequacy of the diet? A case study from an urban area in Mali, West Africa. European Journal of Clinical Nutrition, 52(12), 891.

Health Policy Project. (2016). Health financing profile: South Africa. Retrieved from https://www.healthpolicyproject.com/pubs/7887/SouthAfrica_HFP.pdf

Heckley, G., Gerdtham, U.-G., \& Kjellsson, G. (2016). A general method for decomposing the causes of socioeconomic inequality in health. Journal of health economics, 48, 89-106.

Heisler, M., Bouknight, R. R., Hayward, R. A., Smith, D. M., \& Kerr, E. A. (2002). The relative importance of physician communication, participatory decision making, and patient understanding in diabetes self-management. Journal of general internal medicine, 17(4), 243252.

Higgins, J. P., \& Green, S. (2008). Cochrane handbook for systematic reviews of interventions: Cochrane Collaboration and John Wiley \& Sons Ltd.

Hiscock, R., Bauld, L., Amos, A., Fidler, J. A., \& Munafò, M. (2012). Socioeconomic status and smoking: a review. Annals of the New York Academy of Sciences, 1248(1), 107-123.

Hosseinpoor, A. R., Bergen, N., Kunst, A., Harper, S., Guthold, R., Rekve, D., . . Chatterji, S. (2012). Socioeconomic inequalities in risk factors for non-communicable diseases in lowincome and middle-income countries: results from the World Health Survey. BMC public health, 12(1), 912.

Hsu, J., Flores, G., Evans, D., Mills, A., \& Hanson, K. (2018). Measuring financial protection against catastrophic health expenditures: methodological challenges for global monitoring. International journal for equity in health, 17(1), 69.

Hurlbut, W. B. (2018). Overcoming poverty and inequality in South Africa: An assessment of drivers, constraints and opportunities: World Bank.

International Diabetes Federation. (2001). Diabetes and Cardiovascular Disease: Time to Act: Brussels, Belgium: International Diabetes Federation. 
International Diabetes Federation. (2009). Guideline on Self-Monitoring of Blood Glucose in Non-Insulin Treated Type 2 Diabetes. Brussels, Belgium: International Diabetes Federation.

International Diabetes Federation. (2015). IDF Diabetes Atlas, 7th edition. Brussels, Belgium: International Diabetes Federation.

International Diabetes Federation. (2017). IDF Diabetes Atlas, 8th edition. Brussels, Belgium: International Diabetes Federation.

International Diabetes Federation. (2019). IDF Diabetes Atlas. 9th edition. Brussels, Belgium: International Diabetes Federation.

Ipingbemi, A., \& Erhun, W. (2015). Cost implications of treatment of diabetes mellitus in a secondary healthcare facility in Ibadan. African journal of medicine and medical sciences, 44(1), 79-87.

Jakovljevic, M. B., \& Milovanovic, O. (2015). Growing burden of non-communicable diseases in the emerging health markets: the case of BRICS. Frontiers in public health, 3 (65).

Jakovljevic, M., \& Getzen, T. E. (2016). Growth of global health spending share in low- and middle-income countries. Frontiers in pharmacology, 7 (21).

Jakovljevic, M., \& Ostojic, L. (2013). Comorbidity and multimorbidity in medicine today: challenges and opportunities for bringing separated branches of medicine closer to each other. Psychiatr Danub, 25(Suppl 1), 18-28.

Jing, Z., Chu, J., Imam Syeda, Z., Zhang, X., Xu, Q., Sun, L., \& Zhou, C. (2018). Catastrophic health expenditure among type 2 diabetes mellitus patients: A province-wide study in Shandong, China. Journal of diabetes investigation, 10 (2), 283-289.

Jo, C. (2014). Cost-of-illness studies: concepts, scopes, and methods. Clinical and molecular hepatology, 20(4), 327-337.

Kagee, A., Le Roux, M., \& Dick, J. (2007). Treatment adherence among primary care patients in a historically disadvantaged community in South Africa: A qualitative study. Journal of health psychology, 12(3), 444-460.

Kassahun, T., Gesesew, H., Mwanri, L., \& Eshetie, T. (2016). Diabetes related knowledge, selfcare behaviours and adherence to medications among diabetic patients in Southwest Ethiopia: a cross-sectional survey. BMC endocrine disorders, 16(1), 28.

Kengne, A. P., Amoah, A. G., \& Mbanya, J.-C. (2005). Cardiovascular complications of diabetes mellitus in sub-Saharan Africa. Circulation, 112(23), 3592-3601.

Kengne, A. P., Dzudie, A., \& Sobngwi, E. (2008). Heart failure in sub-Saharan Africa: a literature review with emphasis on individuals with diabetes. Vascular health and risk management, 4(1), 123.

Kengne, A. P., June-Rose Mchiza, Z., Amoah, A. G., \& Mbanya, J. C. (2013). Cardiovascular diseases and diabetes as economic and developmental challenges in Africa. Progress in cardiovascular diseases, 56(3), 302-313 
Kennedy, G. L. (2009). Evaluation of dietary diversity scores for assessment of micronutrient intake and food security in developing countries.

Kessels, R., \& Erreygers, G. (2016). Structural equation modeling for decomposing rankdependent indicators of socioeconomic inequality of health: an empirical study. Health economics review, 6(1), 56.

Khanam, M. A., Streatfield, P. K., Kabir, Z. N., Qiu, C., Cornelius, C., \& Wahlin, Å. (2011). Prevalence and patterns of multimorbidity among elderly people in rural Bangladesh: a crosssectional study. Journal of Health, Population and Nutrition, 406-414.

Kien, V. D., Minh, H. V., Ngoc, N. B., Phuong, T. B., Ngan, T. T., \& Quam, M. B. (2017). Inequalities in Household Catastrophic Health Expenditure and Impoverishment Associated with Non-Communicable Diseases in Chi Linh, Hai Duong, Vietnam. Asia Pacific Journal of Public Health, 29(5_suppl), 35S-44S.

Kien, V. D., Van Minh, H., Giang, K. B., Dao, A., \& Ng, N. (2016). Socioeconomic inequalities in catastrophic health expenditure and impoverishment associated with non-communicable diseases in urban Hanoi, Vietnam. International journal for equity in health, 15(1), 169.

Kimani, D. N., Mugo, M. G., \& Kioko, U. M. (2016). Catastrophic health expenditures and impoverishment in Kenya. European Scientific Journal, 12(15).

Kirigia, J. M., Sambo, H. B., Sambo, L. G., \& Barry, S. P. (2009). Economic burden of diabetes mellitus in the WHO African region. BMC international health and human rights, 9(1), 1.

Kleine-Budde, K., Touil, E., Moock, J., Bramesfeld, A., Kawohl, W., \& Rössler, W. (2014). Cost of illness for bipolar disorder: a systematic review of the economic burden. Bipolar disorders, 16(4), 337-353.

Koch, S. F. (2018). Catastrophic health payments: does the equivalence scale matter? Health policy and planning, 33(8), 966-973.

Kodama, S., Tanaka, S., Heianza, Y., Fujihara, K., Horikawa, C., Shimano, H., . . Sone, H. (2013). Association between physical activity and risk of all-cause mortality and cardiovascular disease in patients with diabetes a meta-analysis. Diabetes Care, 36(2), 471-479.

Krass, I., Schieback, P., \& Dhippayom, T. (2015). Adherence to diabetes medication: a systematic review. Diabetic Medicine, 32(6), 725-737.

Kumar, K., Singh, A., Kumar, S., Ram, F., Singh, A., Ram, U., . . Kowal, P. R. (2015). Socioeconomic differentials in impoverishment effects of out-of-pocket health expenditure in China and India: evidence from WHO SAGE. PloS one, 10(8), e0135051.

Kunna, R., San Sebastian, M., \& Williams, J. S. (2017). Measurement and decomposition of socioeconomic inequality in single and multimorbidity in older adults in China and Ghana: results from the WHO study on global AGEing and adult health (SAGE). International journal for equity in health, 16(1), 79. 
Kwesiga, B., Zikusooka, C. M., \& Ataguba, J. E. (2015). Assessing catastrophic and impoverishing effects of health care payments in Uganda. BMC health services research, 15(1), 30.

Labadarios, D., Steyn, N. P., \& Nel, J. (2011). How diverse is the diet of adult South Africans? Nutrition journal, 10(1), 33.

Labhardt, N. D., Balo, J. R., Ndam, M., Manga, E., \& Stoll, B. (2011). Improved retention rates with low-cost interventions in hypertension and diabetes management in a rural African environment of nurse-led care: a cluster-randomised trial. Tropical Medicine \& International Health, 16(10), 1276-1284.

Larg, A., \& Moss, J. R. (2011). Cost-of-illness studies. Pharmacoeconomics, 29(8), 653-671.

Leśniowska, J., Schubert, A., Wojna, M., Skrzekowska-Baran, I., \& Fedyna, M. (2014). Costs of diabetes and its complications in Poland. The European Journal of Health Economics, 15(6), 653-660.

Levitt, N. S. (2008). Diabetes in Africa: epidemiology, management and healthcare challenges. Heart, 94(11), 1376-1382.

Li, M., Fan, Y., Zhang, X., Hou, W., \& Tang, Z. (2014). Fruit and vegetable intake and risk of type 2 diabetes mellitus: meta-analysis of prospective cohort studies. BMJ open, 4(11), e005497.

Luo, X., Liu, T., Yuan, X., Ge, S., Yang, J., Li, C., \& Sun, W. (2015). Factors influencing selfmanagement in Chinese adults with type 2 diabetes: a systematic review and meta-analysis. International journal of environmental research and public health, 12(9), 11304-11327.

Makate, M., \& Makate, C. (2017). The evolution of socioeconomic status-related inequalities in maternal health care utilization: evidence from Zimbabwe, 1994-2011. Global Health Research and Policy, 2(1), 1.

Manne-Goehler, J., Atun, R., Stokes, A., Goehler, A., Houinato, D., Houehanou, C., . . Balde, N. (2016). Diabetes diagnosis and care in sub-Saharan Africa: pooled analysis of individual data from 12 countries. The Lancet Diabetes \& Endocrinology, 4(11), 903-912.

Mapa-Tassou, C., Katte, J.-C., Maadjhou, C. M., \& Mbanya, J. C. (2019). Economic Impact of Diabetes in Africa. Current diabetes reports, 19(2), 5.

Massyn N, Tanna G, Day C, \& Ndlovu N. (2018). District Health Barometer: District Health Profiles 2017/18. Durban: Health Systems Trust.

Massyn N, Barron P, Day C, \& Ndlovu N, Padarath A. (2019). District Health Barometer: District Health Profiles 2018/19. Durban: Health Systems Trust.

Masupe, T. K., Ndayi, K., Tsolekile, L., Delobelle, P., \& Puoane, T. (2018). Redefining diabetes and the concept of self-management from a patient's perspective: implications for disease risk factor management. Health Educ Res, 33(1), 40-54. 
Mayosi, B. M., \& Benatar, S. R. (2014). Health and health care in South Africa-20 years after Mandela. New England journal of medicine, 371(14), 1344-1353.

Mbanya, J. C. (2007). Diabetes Cost: Sub-Saharan Africa Perspective. International Textbook of Diabetes Mellitus.

Mbanya, J. C. N., Motala, A. A., Sobngwi, E., Assah, F. K., \& Enoru, S. T. (2010). Diabetes in subsaharan africa. The Lancet, 375(9733), 2254-2266.

McGrail, K. M., Van Doorslaer, E., Ross, N. A., \& Sanmartin, C. (2009). Income-related health inequalities in Canada and the United States: a decomposition analysis. American Journal of Public Health, 99(10), 1856-1863.

Mchenga, M., Chirwa, G. C., \& Chiwaula, L. S. (2017). Impoverishing effects of catastrophic health expenditures in Malawi. International journal for equity in health, 16(1), 25.

Mcintyre, D., Thiede, M., Nkosi, M., Mutyambizi, V., Castillo-riquelme, M., Gilson, L., . . . Goudge, J. (2007). SHIELD work package 1 report: a critical analysis of the current South African Health System.

Mendenhall, E., \& Norris, S. A. (2015). Diabetes care among urban women in Soweto, South Africa: a qualitative study. BMC public health, 15(1), 1300.

Miller, T. A., \& DiMatteo, M. R. (2013). Importance of family/social support and impact on adherence to diabetic therapy. Diabetes, metabolic syndrome and obesity: targets and therapy, 6, 421.

Mo, F., Pogany, L. M., Li, F. C., \& Morrison, H. (2006). Prevalence of diabetes and cardiovascular comorbidity in the Canadian Community Health Survey 20022003. The Scientific World Journal, 6, 96-105.

Mogre, V., Abanga, Z. O., Tzelepis, F., Johnson, N. A., \& Paul, C. (2017). Adherence to and factors associated with self-care behaviours in type 2 diabetes patients in Ghana. BMC endocrine disorders, 17(1), 20.

Mohandas, A., Bhasin, S., Upadhyay, M., \& Madhu, S. (2018). Diabetes self-care activities among adults 20 years and above residing in a resettlement colony in East Delhi. Indian Journal of Public Health, 62(2), 104.

Moher, D., Liberati, A., Tetzlaff, J., Altman, D. G., \& Group, P. (2010). Preferred reporting items for systematic reviews and meta-analyses: the PRISMA statement. International journal of surgery, 8(5), 336-341.

Molinier, L., Bauvin, E., Combescure, C., Castelli, C., Rebillard, X., Soulié, M., . . Grosclaude, P. (2008). Methodological considerations in cost of prostate cancer studies: a systematic review. Value in Health, 11(5), 878-885.

Moradi, G., Majdzadeh, R., Mohammad, K., Malekafzali, H., Jafari, S., \& Holakouie-Naieni, K. (2016). Is the status of diabetes socioeconomic inequality changing in Kurdistan province, 
west of Iran? A comparison of two surveys. Medical journal of the Islamic Republic of Iran, 30, 375.

Morisky, D. E., Green, L. W., \& Levine, D. M. (1986). Concurrent and predictive validity of a self-reported measure of medication adherence. Medical care, 67-74.

Moscow Declaration. (2011). The First Global Ministerial Conference on Healthy Lifestyles and Noncommunicable Disease Control. Moscow, 28-29 April 2011. Moscow: WHO, 28-29.

Motala, A., \& Ramaiya, K. (2010). Diabetes: the hidden pandemic and its impact on Subsaharan Africa. Diabetes leadership forum.

Mudzengi, D., Sweeney, S., Hippner, P., Kufa, T., Fielding, K., Grant, A. D., . . Vassall, A. (2017). The patient costs of care for those with TB and HIV: a cross-sectional study from South Africa. Health policy and planning, 32(suppl_4), iv48-iv56.

Mukong, A. K., Van Walbeek, C., \& Ross, H. (2017). Lifestyle and income-related inequality in health in South Africa. International journal for equity in health, 16(1), 103.

Muraki, I., Imamura, F., Manson, J. E., Hu, F. B., Willett, W. C., van Dam, R. M., \& Sun, Q. (2013). Fruit consumption and risk of type 2 diabetes: results from three prospective longitudinal cohort studies. BMJ, 347, f5001.

Murphy, K., Chuma, T., Mathews, C., Steyn, K., \& Levitt, N. (2015). A qualitative study of the experiences of care and motivation for effective self-management among diabetic and hypertensive patients attending public sector primary health care services in South Africa. BMC health services research, 15(1), 303.

Mutowo, M. P., Lorgelly, P. K., Laxy, M., Renzaho, A., Mangwiro, J. C., \& Owen, A. J. (2016). The Hospitalization Costs of Diabetes and Hypertension Complications in Zimbabwe: Estimations and Correlations. J Diabetes Res, 2016.

Mwavua, S. M., Ndungu, E. K., Mutai, K. K., \& Joshi, M. D. (2016). A comparative study of the quality of care and glycemic control among ambulatory type 2 diabetes mellitus clients, at a Tertiary Referral Hospital and a Regional Hospital in Central Kenya. BMC Res Notes, 9(1), 12.

Nadège, C., Valérie, G., Laura, F., Hélène, D.-B., Vanina, B., Olivier, D., . . Laurent, M. (2011). The cost of cochlear implantation: a review of methodological considerations. International journal of otolaryngology, 2011.

Nagl, A., Witte, J., Hodek, J.-M., \& Greiner, W. (2012). Relationship between multimorbidity and direct healthcare costs in an advanced elderly population. Zeitschrift für Gerontologie und Geriatrie, 45(2), 146-154.

National Department of Health. (2011a). National health insurance in South Africa: Policy paper. Pretoria. National Department of Health.

National Department of Health. (2011b). South African Declaration on the Prevention and Control of NonCommunicable Diseases. Pretoria. National Department of Health. 
National Department of Health. (2013). Strategic Plan for the Prevention and Control of NonCommunicable Diseases 2013-17. Pretoria. National Department of Health.

National Department of Health. (2015a). National health insurance for South Africa: Towards universal health coverage. Pretoria. National Department of Health.

National Department of Health. (2015b). User Guide-UPFS 2015. Uniform patient fee schedule for paying patients attending public hospitals. Pretoria. National Department of Health

NCD Risk Factor Collaboration. (2016). Worldwide trends in diabetes since 1980: a pooled analysis of 751 population-based studies with 4 . 4 million participants. The lancet, 387(10027), 1513-1530.

Ncube-Zulu, T., \& Danckwerts, M. P. (2014). Comparative hospitalization cost and length of stay between patients with and without diabetes in a large tertiary hospital in Johannesburg, South Africa. International Journal of Diabetes in Developing Countries, 34(3), 156-162.

Negin, J., Randell, M., Raban, M. Z., Nyirenda, M., Kalula, S., Madurai, L., \& Kowal, P. (2017). Health expenditure and catastrophic spending among older adults living with HIV. Global public health, 12(10), 1282-1296.

Ng, C. S., Lee, J. Y., Toh, M. P., \& Ko, Y. (2014). Cost-of-illness studies of diabetes mellitus: a systematic review. Diabetes research and clinical practice, 105(2), 151-163.

Ngcamphalala, C., \& Ataguba, J. E. (2018). An assessment of financial catastrophe and impoverishment from out-of-pocket health care payments in Swaziland. Global health action, 11(1), 1428473.

Njagi, P., Arsenijevic, J., \& Groot, W. (2018). Understanding variations in catastrophic health expenditure, its underlying determinants and impoverishment in Sub-Saharan African countries: a scoping review. Systematic Reviews, 7(1), 136.

Nthangeni, G., Steyn, N. P., Alberts, M., Steyn, K., Levitt, N. S., Laubscher, R., . . Temple, N. (2002). Dietary intake and barriers to dietary compliance in black type 2 diabetic patients attending primary health-care services. Public health nutrition, 5(2), 329-338.

OANDA. Corporation. Currency converter New York: OANDA Corporation

Obuaku-Igwe, C. C. (2015). Health inequality in South Africa: a systematic review. African Sociological Review, 19(2), 96-131.

O'Donnell, O., O'Neill, S., Van Ourti, T., \& Walsh, B. (2016). conindex: Estimation of concentration indices. The Stata journal, 16(1), 112.

O'Donnell, O., van Doorslaer, E., Adam, W., \& Lindelow, M. (2008). Analyzing Health equity using household survey data. Washington DC: USA: The World Bank.

Ogbera, A., Fasanmade, O., Ohwovoriole, A., \& Adediran, O. (2006). An assessment of the disease burden of foot ulcers in patients with diabetes mellitus attending a teaching hospital in Lagos, Nigeria. The international journal of lower extremity wounds, 5(4), 244-249. 
Ogle, G., Kim, H., Middlehurst, A., Silink, M., \& Jenkins, A. (2015). Financial costs for families of children with Type 1 diabetes in lower-income countries. Diabetic Medicine, 33, 820-826.

Okonta, H. I., Ikombele, J. B., \& Ogunbanjo, G. A. (2014). Knowledge, attitude and practice regarding lifestyle modification in type 2 diabetic patients. African journal of primary health care \& family medicine, 6(1), 1-6.

Okoronkwo, I. L., Ekpemiro, J. N., Okwor, E. U., Okpala, P. U., \& Adeyemo, F. O. (2015). Economic burden and catastrophic cost among people living with type 2 diabetes mellitus attending a tertiary health institution in south-east zone, Nigeria. BMC research notes, $8(1)$, 527.

Oloniniyi, I. O., Akinsulore, A., Aloba, O. O., Mapayi, B. M., Oginni, O. A., \& Makanjuola, R. (2019). Economic cost of schizophrenia in a Nigerian Teaching Hospital. Journal of Neurosciences in Rural Practice, 10(1), 39.

Ong, S. E., Koh, J. J. K., Toh, S.-A. E. S., Chia, K. S., Balabanova, D., McKee, M., . . LegidoQuigley, H. (2018). Assessing the influence of health systems on type 2 diabetes mellitus awareness, treatment, adherence, and control: a systematic review. PloS one, 13(3), e0195086.

Onoka, C. A., Onwujekwe, O. E., Hanson, K., \& Uzochukwu, B. S. (2011). Examining catastrophic health expenditures at variable thresholds using household consumption expenditure diaries. Tropical Medicine \& International Health, 16(10), 1334-1341.

Ording, A. G., \& Sørensen, H. T. (2013). Concepts of comorbidities, multiple morbidities, complications, and their clinical epidemiologic analogs. Clinical epidemiology, 5, 199.

Osborn, C. Y., Bains, S. S., \& Egede, L. E. (2010). Health literacy, diabetes self-care, and glycemic control in adults with type 2 diabetes. Diabetes technology \& therapeutics, 12(11), 913-919.

Pan, A., Wang, Y., Talaei, M., Hu, F. B., \& Wu, T. (2015). Relation of active, passive, and quitting smoking with incident type 2 diabetes: a systematic review and meta-analysis. The Lancet Diabetes \& Endocrinology, 3(12), 958-967.

Patel, M. R., Piette, J. D., Resnicow, K., Kowalski-Dobson, T., \& Heisler, M. (2016). Social determinants of health, cost-related non-adherence, and cost-reducing behaviors among adults with diabetes: findings from the National Health Interview Survey. Medical care, 54(8), 796.

Paterson, B., \& Thorne, S. (2000). Developmental evolution of expertise in diabetes selfmanagement. Clinical Nursing Research, 9(4), 402-419.

Peel, E., Douglas, M., \& Lawton, J. (2007). Self-monitoring of blood glucose in type 2 diabetes: longitudinal qualitative study of patients' perspectives. Bmj, 335(7618), 493.

Phaswana-Mafuya, N., Peltzer, K., Chirinda, W., Musekiwa, A., Kose, Z., Hoosain, E., Davids, A., \& Ramlagan, S. (2013). Self-reported prevalence of chronic non-communicable diseases and associated factors among older adults in South Africa. Global Health Action, 6(1):20936 
Pepper, D., Burch, V., Levitt, N., \& Cleary, S. (2007). Hyperglycaemic emergency admissions to a Secondary-Level hospital-an unnecessary financial burden. Journal of Endocrinology, Metabolism and Diabetes of South Africa, 12(2), 56-60.

Petersen, I., Bhana, A., \& McKay, M. (2005). Sexual violence and youth in South Africa: The need for community-based prevention interventions. Child abuse \& neglect, 29(11), 12331248.

Pinchevsky, Y., Butkow, N., Chirwa, T., \& Raal, F. (2017). Treatment Gaps Found in the Management of Type 2 Diabetes at a Community Health Centre in Johannesburg, South Africa. Journal of diabetes research, 2017.

Qosaj, F. A., Froeschl, G., Berisha, M., Bellaqa, B., \& Holle, R. (2018). Catastrophic expenditures and impoverishment due to out-of-pocket health payments in Kosovo. Cost Effectiveness and Resource Allocation, 16(1), 26.

Quaye, E. A., Amporful, E. O., Akweongo, P., \& Aikins, M. K. (2015). Analysis of the financial cost of diabetes mellitus in four Cocoa clinics of Ghana. Value in Health Regional Issues, 7, 4953.

Ranchod, S., Adams, C., Burger, R., Carvounes, A., Dreyer, K., Smith, A., . . van Biljon, C. (2017). South Africa's hospital sector: old divisions and new developments. South African Health Review, 2017(1), 101-110.

Rarani, M. A., Rashidian, A., Khosravi, A., Arab, M., Abbasian, E., \& Morasae, E. K. (2017). Changes in socio-economic inequality in neonatal mortality in Iran between 1995-2000 and 2005-2010: an Oaxaca decomposition analysis. International journal of health policy and management, 6(4), 219.

Rashad, A. S., \& Sharaf, M. F. (2015). Catastrophic economic consequences of healthcare payments: effects on poverty estimates in Egypt, Jordan, and Palestine. Economies, 3(4), 216234.

Republic of Ghana: Ministry of Health. (2012). National Policy for the Prevention and Control of Chronic Non-Communicable Diseases in Ghana. Ghana. Ministry of Health

Republic of South Africa National Treasury. (2019). Budget review 2019.

Sartorius, B., Sartorius, K., Taylor, M., Aagaard-Hansen, J., Dukhi, N., Day, C., . . . Hofman, K. (2017). Rapidly increasing body mass index among children, adolescents and young adults in a transitioning population, South Africa, 2008-15. International journal of epidemiology, 47(3), 942-952.

SEMDSA Type 2 Diabetes Guidelines Expert Committee. (2017). SEMDSA 2017 guidelines for the management of type 2 diabetes mellitus. J Endocr Metab Diabetes S Afr, 22(1 Suppl 1), S1-S196.

Settumba, S. N., Sweeney, S., Seeley, J., Biraro, S., Mutungi, G., Munderi, P., . . Vassall, A. (2015). The health system burden of chronic disease care: an estimation of provider costs of 
selected chronic diseases in Uganda. Tropical Medicine \& International Health, 20(6), 781790.

Seuring, T., Archangelidi, O., \& Suhrcke, M. (2015). The economic costs of type 2 diabetes: a global systematic review. Pharmacoeconomics, 33(8), 811-831.

Sharma, T., Kalra, J., Dhasmana, D., \& Basera, H. (2014). Poor adherence to treatment: A major challenge in diabetes. JIACM. 2014; 15(1): 26-9

Shaw, B. A., Gallant, M. P., Riley-Jacome, M., \& Spokane, L. S. (2006). Assessing sources of support for diabetes self-care in urban and rural underserved communities. Journal of community health, 31(5), 393-412.

Shilubane, N. (2010). Factors contributing to poor glycaemic control in diabetic patients at Mopani District. Curationis, 33(3), 43-47.

Shisana O, Labadarios D, Rehle T, Simbayi L, Zuma K, Dhansay A, . . . M, F. (2014). The South African National Health and Nutrition Examination Survey: SANHANES-1: HSRC press.

Shrivastava, S. R., Shrivastava, P. S., \& Ramasamy, J. (2013). Role of self-care in management of diabetes mellitus. Journal of diabetes \& Metabolic disorders, 12(1), 14.

Si, Y., Zhou, Z., Su, M., Ma, M., Xu, Y., \& Heitner, J. (2017). Catastrophic healthcare expenditure and its inequality for households with hypertension: evidence from the rural areas of Shaanxi Province in China. International journal for equity in health, 16(1), 27.

Siddiqui, M. A., Bhana, S., \& Daya, R. (2018). The relationship between objectively measured physical activity and parameters of disease control in an African population of type 2 diabetes mellitus. Journal of Endocrinology, Metabolism and Diabetes of South Africa, 23(3), 80-85.

Smith-Spangler, C. M., Bhattacharya, J., \& Goldhaber-Fiebert, J. D. (2012). Diabetes, its treatment, and catastrophic medical spending in 35 developing countries. Diabetes Care, 35(2), 319-326.

Snyder, F. J., \& Flay, B. R. (2012). Brief introduction to the theory of triadic influence. Unpublished manuscript, Purdue University.

Sobngwi, E., Mbanya, J.-C., Unwin, N., Kengne, A. P., Fezeu, L., Minkoulou, E., . . Alberti, K. (2002). Physical activity and its relationship with obesity, hypertension and diabetes in urban and rural Cameroon. International journal of Obesity (26), 8.

Solar, O., \& Irwin, A. (2007). A conceptual framework for action on the social determinants of health.

Solar, O., \& Irwin, A. (2010). A conceptual framework for action on the social determinants of health. Social Determinants of Health Discussion Paper 2 (Policy and Practice). Geneva: WHO.

South African Institute of Race Relations. (2018). Residents of Gauteng metros least reliant on grants [Press release]. Retrieved from https://irr.org.za/media/media-releases 
South African National AIDS Council. (2015). South Africa Global AIDS Response Progress Report (GARPR). WHO and UNAIDS.

South African Revenue Services. (2017). External Guide for employers in respect of employees' tax (2018 tax year) PAYE-GEN-01-G11.

Statistics South Africa. (2015). Mortality and causes of death in South Africa, 2014: Findings from death notification.

Statistics South Africa. (2014). Poverty trends in South Africa: An examination of absolute poverty between 2006 and 2011. Pretoria: Statistics South Africa.

Statistics South Africa. (2017). Mortality and causes of death in South Africa, 2015: Findings from death notification. Pretoria: Statistics South Africa.

Statistics South Africa. (2018a). General Household Survey 2018. Pretoria: Statistics South Africa.

Statistics South Africa. (2018b). Mid-year population estimates. Pretoria: Statistics South Africa.

Statistics South Africa. (2018c). Mortality and causes of death in South Africa, 2016: Findings from death notification. Pretoria: Statistics South Africa.

Statistics South Africa. (2019a). Consumer price index. Statistical release P0141. Pretoria: Statistics South Africa.

Statistics South Africa. (2019b). Quarterly Labour Force Survey. Quarter 2:2019. Statistical release P0211. Pretoria: Statistics South Africa.

Stephani, V., Opoku, D., \& Beran, D. (2018). Self-management of diabetes in Sub-Saharan Africa: a systematic review. BMC public health, 18(1), 1148.

Steyl, T., \& Phillips, J. (2014). Management of type 2 diabetes mellitus: Adherence challenges in environments of low socio-economic status. African journal of primary health care $\&$ family medicine, 6(1), 1-7.

Steyn, N., \& Nel, J. (2006). Dietary intake of adult women in South Africa and Nigeria with a focus on the use of spreads. Tygerberg: South African Medical Research Council.

Steyn, N., Nel, J., Nantel, G., Kennedy, G., \& Labadarios, D. (2006). Food variety and dietary diversity scores in children: are they good indicators of dietary adequacy? Public health nutrition, 9(5), 644-650.

Stokes, A., Berry, K. M., Mchiza, Z., Parker, W.-a., Labadarios, D., Chola, L., . . Rockers, P. C. (2017). Prevalence and unmet need for diabetes care across the care continuum in a national sample of South African adults: Evidence from the SANHANES-1, 2011-2012. PloS one, 12(10), e0184264. 
Stringhini, S., Tabak, A. G., Akbaraly, T. N., Sabia, S., Shipley, M. J., Marmot, M. G., . . Kivimäki, M. (2012). Contribution of modifiable risk factors to social inequalities in type 2 diabetes: prospective Whitehall II cohort study. Bmj, 345, e5452.

Stuhldreher, N., Konnopka, A., Wild, B., Herzog, W., Zipfel, S., Löwe, B., \& König, H. H. (2012). Cost-of-illness studies and cost-effectiveness analyses in eating disorders: A systematic review. International Journal of Eating Disorders, 45(4), 476-491.

Suhrcke, M., Nugent, R. A., Stuckler, D., \& Rocco, L. (2006). Chronic disease: an economic perspective. London: Oxford Health Alliance.

Suleiman, I. A., \& Festus, J. A. (2015). Cost of illness among diabetes mellitus patients in Niger Delta, Nigeria. Journal of Pharmaceutical Health Services Research, 6(1), 53-60.

Suleiman, I., Fadeke, O., \& Okubanjo, O. (2006). Pharmacoeconomic evaluation of antidiabetic therapy in a Nigerian Tertiary Health Institution. Annals of African medicine, 5(3), 132-137.

Sulla, V., \& Zikhali, P. (2018). Overcoming poverty and inequality in South Africa: An assessment of drivers, constraints and opportunities. Washington, DC: World Bank.: World Bank.

Tacconelli, E. (2010). Systematic reviews: CRD's guidance for undertaking reviews in health care. The Lancet Infectious Diseases, 10(4), 226.

Tarricone, R. (2006). Cost-of-illness analysis: what room in health economics? Health Policy, 77(1), 51-63.

Thomson, S., Evetovits, T., Cylus, J., \& Jakab, M. (2016). Monitoring financial protection to assess progress towards universal health coverage in Europe. Public Health Panorama, 2(3), 357-366.

Tiew, K. F., Chan, Y. M., Lye, M. S., \& Loke, S. C. (2014). Factors associated with dietary diversity score among individuals with type 2 diabetes mellitus. Journal of health, population, and nutrition, 32(4), 665.

Ukwaja, K. N., Alobu, I., Abimbola, S., \& Hopewell, P. C. (2013). Household catastrophic payments for tuberculosis care in Nigeria: incidence, determinants, and policy implications for universal health coverage. Infectious diseases of poverty, 2(1), 21.

United Nations General Assembly. (2011). Political declaration of the high-level meeting of the general assembly on the prevention and control of non-communicable diseases, A/66/L. 1. New York: United Nations.

United Nations General Assembly. (2015). Transforming our world: The 2030 agenda for sustainable development.

Vallejo-Torres, L., \& Morris, S. (2010). The contribution of smoking and obesity to incomerelated inequalities in health in England. Social science \& medicine, 71(6), 1189-1198. 
van Dam, H. A., Van der Horst, F., Van den Borne, B., Ryckman, R., \& Crebolder, H. (2003). Provider-patient interaction in diabetes care: effects on patient self-care and outcomes: a systematic review. Patient education and counseling, 51(1), 17-28.

Van Rooijen, A., Rheeder, P., Eales, C., \& Molatoli, H. (2002). Barriers to and expectations of performing physical activity in female subjects with type 2 diabetes. South African Journal of Physiotherapy, 58(1), 3-11.

Vellakkal, S., Millett, C., Basu, S., Khan, Z., Aitsi-Selmi, A., Stuckler, D., \& Ebrahim, S. (2015). Are estimates of socioeconomic inequalities in chronic disease artefactually narrowed by selfreported measures of prevalence in low-income and middle-income countries? Findings from the WHO-SAGE survey. J Epidemiol Community Health, 69(3), 218-225.

Vellakkal, S., Subramanian, S., Millett, C., Basu, S., Stuckler, D., \& Ebrahim, S. (2013). Socioeconomic inequalities in non-communicable diseases prevalence in India: disparities between self-reported diagnoses and standardized measures. PloS one, 8(7), e68219.

Volmink, H. C., Bertram, M. Y., Jina, R., Wade, A. N., \& Hofman, K. J. (2014). Applying a private sector capitation model to the management of type 2 diabetes in the South African public sector: a cost-effectiveness analysis. BMC health services research, 14(1), 444.

Wagstaff, A. (2002). Poverty and health sector inequalities. Bulletin of the World Health Organisation, 80, 97-105.

Wagstaff, A., \& Eozenou, P. H.-V. (2014). CATA meets IMPOV: a unified approach to measuring financial protection in health. The World Bank.

Wagstaff, A., \& van Doorslaer, E. (2003). Catastrophe and impoverishment in paying for health care: with applications to Vietnam 1993-1998. Health economics, 12(11), 921-933.

Wagstaff, A., Van Doorslaer, E., \& Watanabe, N. (2003). On decomposing the causes of health sector inequalities with an application to malnutrition inequalities in Vietnam. Journal of econometrics, 112(1), 207-223.

Wang, H., Naghavi, M., Allen, C., Barber, R. M., Bhutta, Z. A., Carter, A., . . Murray, C. J. L. (2016). Global, regional, and national life expectancy, all-cause mortality, and cause-specific mortality for 249 causes of death, 1980-2015: a systematic analysis for the Global Burden of Disease Study 2015. The Lancet, 388(10053), 1459-1544. doi:10.1016/s0140-6736(16)310121

Williams, E. D., Tapp, R. J., Magliano, D., Shaw, J. E., Zimmet, P., Z, \& Oldenburg, B. F. (2010). Health behaviours, socioeconomic status and diabetes incidence: The Australian Diabetes Obesity and Lifestyle Study (AusDiab). Diabetologia, 53(12), 2538-2545.

Williams, G. C., Freedman, Z. R., \& Deci, E. L. (1998). Supporting autonomy to motivate patients with diabetes for glucose control. Diabetes Care, 21(10), 1644-1651.

World Health Organisation. (1990). Diet, nutrition, and the prevention of chronic diseases. Report of a WHO Study Group. Diet, nutrition, and the prevention of chronic diseases. Report of a WHO Study Group. (797). 
World Health Organisation. (2003). Food and Agriculture Organization: Diet, nutrition and the prevention of chronic diseases, Report of a Joint WHO/FAO Expert Consultation. Geneva, Switzerland: WHO.

World Health Organisation. (2010a). The World Health Report: Health Systems Financing: The Path to Universal Coverage Geneva.

World Health Organisation. (2010b). Global recommendations on physical activity for health. Geneva: World Health Organisation.

World Health Organisation. (2011). Global status report on noncommunicable diseases 2010: Geneva: World Health Organisation.

World Health Organisation. (2012). Global Physical Activity Questionnaire (GPAQ) -analysis guide.

World Health Organisation. (2013). Global Action Plan for the Prevention and Control of Noncommunicable Diseases: 2013-2020.

World Health Organisation. (2014a). Global status report on noncommunicable diseases 2014: Geneva: World Health Organisation.

World Health Organisation. (2014b). WHO African Region Expenditure Atlas, November 2014.

World Health Organisation. (2014c). WHO global health expenditure atlas. September 2014. . In WHO Global Health Expenditure Atlas. Switzerland.

World Health Organisation. (2016a). Global Report on Diabetes. Geneva: World Health Organisation.

World Health Organisation. (2016b). Health financing profile: South Africa. Retrieved 24 July 2019 https://www.healthpolicyproject.com/pubs/7887/SouthAfrica_HFP.pdf

World Health Organisation and the World Bank. (2017). World Bank. Tracking universal health coverage: 2017 global monitoring report. Geneva: World Health Organisation.

Xiao-Ren, P., Guang-Wei, L., Ying-Hua, H., Ji-Xing, W., \& et al. (1997). Effects of diet and exercise in preventing NIDDM in people with impaired glucose tolerance: The Da Qing IGT and diabetes study. Diabetes Care, 20(4), 537-544.

Xin Feng, M., Manns, B. J., \& Campbell, D. J. (2018). Financial Barriers for Patients with Diabetes: What Providers Can Do to Help. Arratum: The Diabetes Communicator.

Xu, K. (2005). Distribution of health payments and catastrophic expenditures methodology. In Distribution of health payments and catastrophic expenditures Methodology.

Xu, K., Evans, D. B., Kawabata, K., Zeramdini, R., Klavus, J., \& Murray, C. J. (2003). Household catastrophic health expenditure: a multicountry analysis. The lancet, 362(9378), 111-117.

Xu, Y., Pan, W., \& Liu, H. (2010). Self-management practices of Chinese Americans with type 2 diabetes. Nursing \& health sciences, 12(2), 228-234. 
Yesudian, C. A., Grepstad, M., Visintin, E., \& Ferrario, A. (2014). The economic burden of diabetes in India: a review of the literature. Globalization and health, 10(1), 80.

Yiengprugsawan, V., Lim, L. L., Carmichael, G. A., Dear, K. B., \& Sleigh, A. C. (2010). Decomposing socioeconomic inequality for binary health outcomes: an improved estimation that does not vary by choice of reference group. BMC research notes, 3(1), 57.

Zemlin, A. E., Matsha, T. E., Hassan, M. S., \& Erasmus, R. T. (2011). HbA1c of 6.5\% to diagnose diabetes

mellitus-does it work for us? - the bellville South Africa study. PloS one, 6(8).

Zhang, X., Decker, F. H., Luo, H., Geiss, L. S., Pearson, W. S., Saaddine, J. B., . . Albright, A. (2010). Trends in the prevalence and comorbidities of diabetes mellitus in nursing home residents in the United States: 1995-2004. Journal of the American Geriatrics Society, 58(4), 724-730. 


\section{Appendix A: Pubmed search strategy}

Related to Chapter 2

((("diabetes mellitus"[MeSH Terms] OR ("diabetes"[All Fields] AND "mellitus"[All Fields]) OR "diabetes mellitus"[All Fields] OR "diabetes"[All Fields] OR "diabetes insipidus"[MeSH Terms] OR ("diabetes"[All Fields] AND "insipidus"[All Fields]) OR "diabetes insipidus"[All Fields]) OR "diabetes mellitus"[All Fields] OR diabetics[All Fields]) AND (("economics"[Subheading] OR "economics"[All Fields] OR "cost"[All Fields] OR "costs and cost analysis"[MeSH Terms] OR ("costs"[All Fields] AND "cost"[All Fields] AND "analysis"[All Fields]) OR "costs and cost analysis"[All Fields]) OR "cost of illness"[All Fields] OR "economic burden"[All Fields] OR ("health expenditures"[MeSH Terms] OR ("health"[All Fields] AND "expenditures"[All Fields]) OR "health expenditures"[All Fields] OR "expenditure"[All Fields]) OR "healthcare costs"[All Fields])) AND (("africa"[MeSH Terms] OR "africa"[All Fields]) OR ("africa south of the sahara"[MeSH Terms] OR ("africa"[All Fields] AND "south"[All Fields] AND "sahara"[All Fields]) OR "africa south of the sahara"[All Fields] OR ("sub"[All Fields] AND "saharan"[All Fields] AND "africa"[All Fields]) OR "sub saharan africa"[All Fields]) OR ("angola"[MeSH Terms] OR "angola"[All Fields]) OR ("benin"[MeSH Terms] OR "benin"[All Fields]) OR ("botswana"[MeSH Terms] OR "botswana"[All Fields]) OR ("burkina faso"[MeSH Terms] OR ("burkina"[All Fields] AND "faso"[All Fields]) OR "burkina faso"[All Fields]) OR ("burundi"[MeSH Terms] OR "burundi"[All Fields]) OR ("cape verde"[MeSH Terms] OR ("cape"[All Fields] AND "verde"[All Fields]) OR "cape verde"[All Fields] OR ("cabo"[All Fields] AND "verde"[All Fields]) OR "cabo verde"[All Fields]) OR ("cameroon"[MeSH Terms] OR "cameroon"[All Fields]) OR ("central african republic"[MeSH Terms] OR ("central"[All Fields] AND "african"[All Fields] AND "republic"[All Fields]) OR "central african republic"[All Fields]) OR ("chad"[MeSH Terms] OR "chad"[All Fields]) OR ("comoros"[MeSH Terms] OR "comoros"[All Fields]) OR ("congo"[MeSH Terms] OR "congo"[All Fields]) OR ("cote d'ivoire"[MeSH Terms] OR ("cote"[All Fields] AND "d'ivoire"[All Fields]) OR "cote d'ivoire"[All Fields]) OR ("democratic republic of the congo"[MeSH Terms] OR ("democratic"[All Fields] AND "republic"[All Fields] AND "congo"[All Fields]) OR "democratic republic of the congo"[All Fields]) OR ("djibouti"[MeSH Terms] OR "djibouti"[All Fields]) OR ("egypt"[MeSH Terms] OR "egypt"[All Fields]) OR ("equatorial guinea"[MeSH Terms] OR ("equatorial"[All Fields] AND "guinea"[All Fields]) OR "equatorial guinea"[All Fields]) OR ("eritrea"[MeSH Terms] OR "eritrea"[All Fields]) OR ("ethiopia"[MeSH Terms] OR "ethiopia"[All Fields]) OR ("gabon"[MeSH Terms] OR "gabon"[All Fields]) OR ("gambia"[MeSH Terms] OR "gambia"[All Fields]) OR ("ghana"[MeSH Terms] OR "ghana"[All Fields]) OR ("guinea"[MeSH Terms] OR "guinea"[All Fields]) OR ("guinea-bissau"[MeSH Terms] OR "guinea-bissau"[All Fields] OR ("guinea"[All Fields] AND "bissau"[All Fields]) OR "guinea bissau"[All Fields]) OR ("kenya"[MeSH Terms] OR "kenya"[All Fields]) OR ("lesotho"[MeSH Terms] OR "lesotho"[All Fields]) OR ("liberia"[MeSH Terms] OR "liberia"[All Fields]) OR ("libya"[MeSH Terms] OR "libya"[All Fields]) OR ("madagascar"[MeSH Terms] OR "madagascar"[All Fields]) OR ("malawi"[MeSH Terms] OR "malawi"[All Fields]) OR ("mali"[MeSH Terms] OR "mali"[All Fields]) OR ("mauritania"[MeSH Terms] OR 
"mauritania"[All Fields]) OR ("mauritius"[MeSH Terms] OR "mauritius"[All Fields]) OR ("comoros"[MeSH Terms] OR "comoros"[All Fields] OR "mayotte"[All Fields]) OR ("morocco"[MeSH Terms] OR "morocco"[All Fields]) OR ("mozambique"[MeSH Terms] OR "mozambique"[All Fields]) OR ("namibia"[MeSH Terms] OR "namibia"[All Fields]) OR ("niger"[MeSH Terms] OR "niger"[All Fields]) OR ("nigeria"[MeSH Terms] OR "nigeria"[All Fields]) OR ("reunion"[MeSH Terms] OR "reunion"[All Fields]) OR ("rwanda"[MeSH Terms] OR "rwanda"[All Fields]) OR ("atlantic islands"[MeSH Terms] OR ("atlantic"[All Fields] AND "islands"[All Fields]) OR "atlantic islands"[All Fields] OR ("saint"[All Fields] AND "helena"[All Fields]) OR "saint helena"[All Fields]) OR ("atlantic islands"[MeSH Terms] OR ("atlantic"[All Fields] AND "islands"[All Fields]) OR "atlantic islands"[All Fields] OR ("sao"[All Fields] AND "tome"[All Fields] AND "principe"[All Fields]) OR "sao tome and principe"[All Fields]) OR ("senegal"[MeSH Terms] OR "senegal"[All Fields]) OR ("seychelles"[MeSH Terms] OR "seychelles"[All Fields]) OR ("sierra leone"[MeSH Terms] OR ("sierra"[All Fields] AND "leone"[All Fields]) OR "sierra leone"[All Fields]) OR ("somalia"[MeSH Terms] OR "somalia"[All Fields]) OR ("south africa"[MeSH Terms] OR ("south"[All Fields] AND "africa"[All Fields]) OR "south africa"[All Fields]) OR ("south sudan"[MeSH Terms] OR ("south"[All Fields] AND "sudan"[All Fields]) OR "south sudan"[All Fields]) OR ("sudan"[MeSH Terms] OR "sudan"[All Fields]) OR ("swaziland"[MeSH Terms] OR "swaziland"[All Fields]) OR ("tanzania"[MeSH Terms] OR "tanzania"[All Fields]) OR ("togo"[MeSH Terms] OR "togo"[All Fields]) OR ("tunisia"[MeSH Terms] OR "tunisia"[All Fields]) OR ("uganda"[MeSH Terms] OR "uganda"[All Fields]) OR ("zambia"[MeSH Terms] OR "zambia"[All Fields]) OR ("zimbabwe"[MeSH Terms] OR "zimbabwe"[All Fields])) AND (("2006/01/01"[PDAT] : "2016/12/31"[PDAT]) AND English[lang]) 


\section{Appendix B: PRISMA Checklist}

\section{Related to Chapter 2}

\begin{tabular}{|c|c|c|c|}
\hline Section/topic & \# & Checklist item & $\begin{array}{l}\text { Reported in } \\
\text { section }\end{array}$ \\
\hline \multicolumn{4}{|l|}{ TITLE } \\
\hline Title & 1 & Identify the report as a systematic review, meta-analysis, or both. & Title \\
\hline Structured summary & 2 & $\begin{array}{l}\text { Provide a structured summary including, as applicable: background; objectives; data sources; study } \\
\text { eligibility criteria, participants, and interventions; study appraisal and synthesis methods; results; } \\
\text { limitations; conclusions and implications of key findings; systematic review registration number. }\end{array}$ & Abstract \\
\hline Rationale & 3 & Describe the rationale for the review in the context of what is already known. & Introduction \\
\hline Objectives & 4 & $\begin{array}{l}\text { Provide an explicit statement of questions being addressed with reference to participants, } \\
\text { interventions, comparisons, outcomes, and study design (PICOS). }\end{array}$ & $\begin{array}{l}\text { Inclusion } \\
\text { criteria }\end{array}$ \\
\hline \multicolumn{4}{|l|}{ METHODS } \\
\hline Protocol and registration & 5 & $\begin{array}{l}\text { Indicate if a review protocol exists, if and where it can be accessed (e.g., Web address), and, if } \\
\text { available, provide registration information including registration number. }\end{array}$ & $\begin{array}{l}\text { Not } \\
\text { registered }\end{array}$ \\
\hline Eligibility criteria & 6 & $\begin{array}{l}\text { Specify study characteristics (e.g., PICOS, length of follow-up) and report characteristics (e.g., years } \\
\text { considered, language, publication status) used as criteria for eligibility, giving rationale. }\end{array}$ & $\begin{array}{l}\text { Inclusion } \\
\text { criteria }\end{array}$ \\
\hline Search & 8 & $\begin{array}{l}\text { Present full electronic search strategy for at least one database, including any limits used, such } \\
\text { that it could be repeated. }\end{array}$ & Appendix A \\
\hline Study selection & 9 & $\begin{array}{l}\text { State the process for selecting studies (i.e., screening, eligibility, included in systematic review, } \\
\text { and, if applicable, included in the meta-analysis). }\end{array}$ & $\begin{array}{l}\text { Inclusion } \\
\text { criteria }\end{array}$ \\
\hline Data collection process & 10 & $\begin{array}{l}\text { Describe method of data extraction from reports (e.g., piloted forms, independently, in duplicate) } \\
\text { and any processes for obtaining and confirming data from investigators. }\end{array}$ & $\begin{array}{l}\text { Extraction of } \\
\text { information }\end{array}$ \\
\hline Data items & 11 & $\begin{array}{l}\text { List and define all variables for which data were sought (e.g., PICOS, funding sources) and any } \\
\text { assumptions and simplifications made. }\end{array}$ & $\begin{array}{l}\text { Inclusion } \\
\text { criteria and } \\
\text { extraction of } \\
\text { information }\end{array}$ \\
\hline $\begin{array}{l}\text { Risk of bias in individual } \\
\text { studies }\end{array}$ & 12 & $\begin{array}{l}\text { Describe methods used for assessing risk of bias of individual studies (including specification of } \\
\text { whether this was done at the study or outcome level), and how this information is to be used in } \\
\text { any data synthesis. }\end{array}$ & $\begin{array}{l}\text { Quality of } \\
\text { included } \\
\text { studies }\end{array}$ \\
\hline $\begin{array}{l}\text { Risk of bias across } \\
\text { studies }\end{array}$ & 15 & $\begin{array}{l}\text { Specify any assessment of risk of bias that may affect the cumulative evidence (e.g., publication } \\
\text { bias, selective reporting within studies). }\end{array}$ & $\begin{array}{l}\text { Quality of } \\
\text { included } \\
\text { studies }\end{array}$ \\
\hline Additional analyses & 16 & $\begin{array}{l}\text { Describe methods of additional analyses (e.g., sensitivity or subgroup analyses, meta-regression), if } \\
\text { done, indicating which were pre-specified. }\end{array}$ & $\begin{array}{l}\text { Not } \\
\text { performed }\end{array}$ \\
\hline \multicolumn{4}{|c|}{ ( } \\
\hline Study selection & 17 & $\begin{array}{l}\text { Give numbers of studies screened, assessed for eligibility, and included in the review, with reasons } \\
\text { for exclusions at each stage, ideally with a flow diagram. }\end{array}$ & $\begin{array}{l}\text { Results and } \\
\text { flow chart }\end{array}$ \\
\hline Study characteristics & 18 & $\begin{array}{l}\text { For each study, present characteristics for which data were extracted (e.g., study size, PICOS, } \\
\text { follow-up period) and provide the citations. }\end{array}$ & $\begin{array}{l}\text { General } \\
\text { characteristic } \\
\text { s of included } \\
\text { studies }\end{array}$ \\
\hline $\begin{array}{l}\text { Risk of bias within } \\
\text { studies }\end{array}$ & 19 & $\begin{array}{l}\text { Present data on risk of bias of each study and, if available, any outcome level assessment (see item } \\
\text { 12). }\end{array}$ & $\begin{array}{l}\text { Quality of } \\
\text { included } \\
\text { studies }\end{array}$ \\
\hline $\begin{array}{l}\text { Results of individual } \\
\text { studies }\end{array}$ & 20 & $\begin{array}{l}\text { For all outcomes considered (benefits or harms), present, for each study: (a) simple summary data } \\
\text { for each intervention group (b) effect estimates and confidence intervals, ideally with a forest plot. }\end{array}$ & $\begin{array}{l}\text { Table } 2-2 \\
\text { Table } 2-3\end{array}$ \\
\hline Synthesis of results & 21 & $\begin{array}{l}\text { Present results of each meta-analysis done, including confidence intervals and measures of } \\
\text { consistency. }\end{array}$ & $\begin{array}{l}\text { Not } \\
\text { performed }\end{array}$ \\
\hline $\begin{array}{l}\text { Risk of bias across } \\
\text { studies }\end{array}$ & 22 & Present results of any assessment of risk of bias across studies (see Item 15). & $\begin{array}{l}\text { Quality of } \\
\text { included } \\
\text { studies }\end{array}$ \\
\hline Additional analysis & 23 & $\begin{array}{l}\text { Give results of additional analyses, if done (e.g., sensitivity or subgroup analyses, meta-regression } \\
\text { [see Item 16]). }\end{array}$ & Appendix D \\
\hline \multicolumn{4}{|l|}{ DISCUSSION } \\
\hline Summary of evidence & 24 & $\begin{array}{l}\text { Summarize the main findings including the strength of evidence for each main outcome; consider } \\
\text { their relevance to key groups (e.g., health care providers, users, and policy makers). }\end{array}$ & Discussion \\
\hline
\end{tabular}




\begin{tabular}{|l|l|l|l|}
\hline Section/topic & $\#$ & Checklist item & $\begin{array}{l}\text { Reported in } \\
\text { section }\end{array}$ \\
\hline Limitations & 25 & $\begin{array}{l}\text { Discuss limitations at study and outcome level (e.g., risk of bias), and at review-level (e.g., } \\
\text { incomplete retrieval of identified research, reporting bias). }\end{array}$ & Limitations \\
\hline Conclusions & 26 & $\begin{array}{l}\text { Provide a general interpretation of the results in the context of other evidence, and implications } \\
\text { for future research. }\end{array}$ & Conclusion \\
\hline FUNDING & 27 & $\begin{array}{l}\text { Describe sources of funding for the systematic review and other support (e.g., supply of data); role } \\
\text { of funders for the systematic review. }\end{array}$ & Funding \\
\hline Funding &
\end{tabular}




\section{Appendix C: Articles included in review in alphabetical order:}

Related to Chapter 2

1. Abdulganiyu G, Fola T. What is the cost of illness of type II Diabetes Mellitus in a Developing Economy. Int J Pharm Pharm Sci. 2014;6:927-31.

2. Alouki K, Delisle H, Besançon S, Baldé N, Sidibé-Traoré A, Drabo J, et al. Simple calculator to estimate the medical cost of diabetes in sub-Saharan Africa. World journal of diabetes. 2015;6(16):1312.

3. Basu S, Shankar V, Yudkin JS. Comparative effectiveness and cost-effectiveness of treat-to-target versus benefit-based tailored treatment of type 2 diabetes in low-income and middle-income countries: a modelling analysis. The Lancet Diabetes \& Endocrinology. 2016;4(11):922-32.

4. Boutayeb W, Lamlili ME, Boutayeb A, Boutayeb S. Estimation of direct and indirect cost of diabetes in Morocco. 2013.

5. Bovet P, Shamlaye C, Gabriel A, Riesen W, Paccaud F. Prevalence of cardiovascular risk factors in a middle-income country and estimated cost of a treatment strategy. BMC Public health. 2006;6(1):9.

6. Cavanagh P, Attinger C, Abbas Z, Bal A, Rojas N, Xu ZR. Cost of treating diabetic foot ulcers in five different countries. Diabetes/metabolism research and reviews. 2012;28(S1):107-11.

7. Danmusa UM, Terhile I, Nasir IA, Ahmad AA, Muhammad HY. Prevalence and healthcare costs associated with the management of diabetic foot ulcer in patients attending Ahmadu Bello University Teaching Hospital, Nigeria. International journal of health sciences. 2016;10(2):219.

8. Elrayah-Eliadarous $\mathrm{H}$, Yassin $\mathrm{K}$, Eltom M, Abdelrahman S, Wahlström R, Östenson C-

G. Direct costs for care and glycaemic control in patients with type 2 diabetes in Sudan. Experimental and clinical endocrinology \& diabetes. 2010;118(04):220-5.

9. Enwere O, Salako B, Falade C. Prescription and cost consideration at a diabetic clinic in Ibadan, Nigeria: A report. Annals of Ibadan postgraduate medicine. 2006;4(2).

10. Fadare J, Olamoyegun M, Gbadegesin B. Medication adherence and direct treatment cost among diabetes patients attending a tertiary healthcare facility in Ogbomosho, Nigeria. Malawi Medical Journal. 2015;27(2):65-70. 
11. Feleke $\mathrm{Y}$, Enquselassie F. Cost of hospitalization of diabetic patients admitted at Tikur Anbessa Specialized Hospital, Addis Ababa, Ethiopia. Ethiopian medical journal. 2007;45(3):275-82.

12. Ipingbemi A, Erhun W. Cost implications of treatment of diabetes mellitus in a secondary healthcare facility in Ibadan. African journal of medicine and medical sciences. 2015;44(1):79-87.

13. Kirigia JM, Sambo HB, Sambo LG, Barry SP. Economic burden of diabetes mellitus in the WHO African region. BMC international health and human rights. 2009;9(1):1.

14. Labhardt ND, Balo JR, Ndam M, Manga E, Stoll B. Improved retention rates with lowcost interventions in hypertension and diabetes management in a rural African environment of nurse-led care: a cluster-randomised trial. Tropical Medicine \& International Health. 2011;16(10):1276-84.

15. Mutowo MP, Lorgelly PK, Laxy M, Renzaho A, Mangwiro JC, Owen AJ. The Hospitalization Costs of Diabetes and Hypertension Complications in Zimbabwe: Estimations and Correlations. Journal of diabetes research. 2016;2016.

16. Mwavua SM, Ndungu EK, Mutai KK, Joshi MD. A comparative study of the quality of care and glycemic control among ambulatory type 2 diabetes mellitus clients, at a Tertiary Referral Hospital and a Regional Hospital in Central Kenya. BMC research notes. 2016;9(1):12.

17. Ncube-Zulu T, Danckwerts MP. Comparative hospitalization cost and length of stay between patients with and without diabetes in a large tertiary hospital in Johannesburg, South Africa. International Journal of Diabetes in Developing Countries. 2014;34(3):156-62. 18. Ogbera A, Fasanmade O, Ohwovoriole A, Adediran O. An assessment of the disease burden of foot ulcers in patients with diabetes mellitus attending a teaching hospital in Lagos, Nigeria. The international journal of lower extremity wounds. 2006;5(4):244-9. 19. Ogle G, Kim H, Middlehurst A, Silink M, Jenkins A. Financial costs for families of children with Type 1 diabetes in lower-income countries. Diabetic Medicine. 2015.

20. Okoronkwo IL, Ekpemiro JN, Okwor EU, Okpala PU, Adeyemo FO. Economic burden and catastrophic cost among people living with type 2 diabetes mellitus attending a tertiary health institution in south-east zone, Nigeria. BMC research notes. 2015;8(1):527. 
21. Pepper D, Levitt N, Cleary S, Burch V. Hyperglycaemic emergency admissions to a secondary-level hospital-an unnecessary financial burden: original article. South African Medical Journal. 2007;97(10):963-7.

22. Quaye EA, Amporful EO, Akweongo P, Aikins MK. Analysis of the financial cost of diabetes mellitus in four Cocoa clinics of Ghana. Value in Health Regional Issues. 2015;7:4953.

23. Settumba SN, Sweeney S, Seeley J, Biraro S, Mutungi G, Munderi P, et al. The health system burden of chronic disease care: an estimation of provider costs of selected chronic diseases in Uganda. Tropical medicine \& international health. 2015;20(6):781-90.

24. Suleiman I, Fadeke O, Okubanjo O. Pharmacoeconomic evaluation of anti-diabetic therapy in a Nigerian Tertiary Health Institution. Annals of African medicine. 2006;5(3):1327.

25. Suleiman IA, Festus JA. Cost of illness among diabetes mellitus patients in Niger Delta, Nigeria. Journal of Pharmaceutical Health Services Research. 2015;6(1):53-60.

26. Volmink HC, Bertram MY, Jina R, Wade AN, Hofman KJ. Applying a private sector capitation model to the management of type 2 diabetes in the South African public sector: a cost-effectiveness analysis. BMC health services research. 2014;14(1):444. 
Appendix D: Cost ratio for individuals with and without diabetes mellitus complications Related to Chapter 2

\begin{tabular}{|c|c|c|c|c|c|c|c|}
\hline Ref & Complication & Country & $\begin{array}{l}\text { Diabetes } \\
\text { type }\end{array}$ & $\begin{array}{l}\text { Complicated } \\
\text { diabetes }\end{array}$ & $\begin{array}{l}\text { Uncomplicated } \\
\text { diabetes }\end{array}$ & $\begin{array}{l}\text { Cost } \\
\text { ratio }\end{array}$ & Average \\
\hline 2 & Diabetic foot & Benin & 2 & 1880 & 574.3 & 3.27 & \multirow{4}{*}{3.78} \\
\hline 2 & Diabetic foot & Burkina Faso & 2 & 2588.83 & 628.27 & 4.12 & \\
\hline 2 & Diabetic foot & Guinea & 2 & 93.08 & 22.25 & 4.18 & \\
\hline 2 & Diabetic foot & Mali & 2 & 1327.32 & 375.71 & 3.53 & \\
\hline 25 & Hypertension & Nigeria & 1 & 1491.87 & 1307.83 & 1.14 & \multirow{6}{*}{1.80} \\
\hline 25 & Hypertension & Nigeria & 2 & 779.31 & 595.28 & 1.31 & \\
\hline 2 & Hypertension & Benin & 2 & 934.58 & 574.3 & 1.63 & \\
\hline 2 & Hypertension & Burkina Faso & 2 & 1057.41 & 628.27 & 1.68 & \\
\hline 2 & Hypertension & Guinea & 2 & 64.82 & 22.25 & 2.91 & \\
\hline 2 & Hypertension & Mali & 2 & 803.52 & 375.71 & 2.14 & \\
\hline 25 & $\begin{array}{l}\text { Hypertension and } \\
\text { Cholesterol }\end{array}$ & Nigeria & 1 & 2303.77 & 1307.83 & 1.76 & \multirow{2}{*}{2.24} \\
\hline 25 & $\begin{array}{l}\text { Hypertension and } \\
\text { Cholesterol }\end{array}$ & Nigeria & 2 & 1623.8 & 595.28 & 2.73 & \\
\hline 2 & Infected diabetic foot & Benin & 2 & 1972.11 & 574.3 & 3.43 & \multirow{4}{*}{4.27} \\
\hline 2 & Infected diabetic foot & Burkina Faso & 2 & 2044.7 & 628.27 & 3.25 & \\
\hline 2 & Infected diabetic foot & Guinea & 2 & 117.8 & 22.25 & 5.29 & \\
\hline 2 & Infected diabetic foot & Mali & 2 & 1914.2 & 375.71 & 5.09 & \\
\hline 2 & Ischemic heart disease & Benin & 2 & 1449.28 & 574.3 & 2.52 & \multirow{4}{*}{2.61} \\
\hline 2 & Ischemic heart disease & Burkina Faso & 2 & 1391.18 & 628.27 & 2.21 & \\
\hline 2 & Ischemic heart disease & Guinea & 2 & 55.28 & 22.25 & 2.48 & \\
\hline 2 & Ischemic heart disease & Mali & 2 & 1209.4 & 375.71 & 3.22 & \\
\hline 2 & Keto acidosis & Benin & 2 & 1099.83 & 574.3 & 1.92 & \multirow{4}{*}{1.79} \\
\hline 2 & Keto acidosis & Burkina Faso & 2 & 1085.46 & 628.27 & 1.73 & \\
\hline 2 & Keto acidosis & Guinea & 2 & 39.21 & 22.25 & 1.76 & \\
\hline 2 & Keto acidosis & Mali & 2 & 666.4 & 375.71 & 1.77 & \\
\hline 2 & Nephropathy & Benin & 2 & 2302.6 & 574.3 & 4.01 & \multirow{4}{*}{4.38} \\
\hline 2 & Nephropathy & Burkina Faso & 2 & 2196.15 & 628.27 & 3.50 & \\
\hline 2 & Nephropathy & Guinea & 2 & 142.71 & 22.25 & 6.41 & \\
\hline 2 & Nephropathy & Mali & 2 & 1346.52 & 375.71 & 3.58 & \\
\hline 2 & Retinopathy & Benin & 2 & 866.86 & 574.3 & 1.51 & \multirow{4}{*}{1.41} \\
\hline 2 & Retinopathy & Burkina Faso & 2 & 858.27 & 628.27 & 1.37 & \\
\hline 2 & Retinopathy & Guinea & 2 & 33.03 & 22.25 & 1.48 & \\
\hline 2 & Retinopathy & Mali & 2 & 485.41 & 375.71 & 1.29 & \\
\hline 2 & Stroke & Benin & 2 & 631.18 & 574.3 & 1.10 & \multirow{4}{*}{1.08} \\
\hline 2 & Stroke & Burkina Faso & 2 & 675.96 & 628.27 & 1.08 & \\
\hline 2 & Stroke & Guinea & 2 & 23.84 & 22.25 & 1.07 & \\
\hline 2 & Stroke & Mali & 2 & 403.13 & 375.71 & 1.07 & \\
\hline 2 & Acute Stroke & Benin & 2 & 1725.58 & 574.3 & 3.00 & \multirow{4}{*}{15.72} \\
\hline 2 & Acute Stroke & Burkina Faso & 2 & 1615.56 & 628.27 & 2.57 & \\
\hline 2 & Acute Stroke & Guinea & 2 & 1247.79 & 22.25 & 56.08 & \\
\hline 2 & Acute Stroke & Mali & 2 & 455 & 375.71 & 1.21 & \\
\hline
\end{tabular}




\section{Appendix E: Participant consent form-Quantitative data}

Related to Chapter 3, 5 and 6

Title of study: Costs and management of diabetes among South African adults

\section{Who we are}

Hello, my name is I am working for the Human Sciences Research Council.

We are conducting research on the Costs and management of diabetes among South African adults. We are conducting a study to establish the impact of diabetes on households and individuals and also determine the factors associated with adherence to medication and lifestyle modification.

\section{Your participation}

We are asking you whether you will complete a questionnaire that asks about your demographic characteristics, expenditures made towards health care, experiences with diabetic health care, impact on employment and adherence to diabetic medication and lifestyle modification. If you agree, a questionnaire will be administered by a field worker and will take approximately 30 minutes to complete. Please understand that your participation is voluntary and you are not being forced to take part in this study. The choice of whether to participate or not, is yours alone. If you choose not to take part, you will not be affected in any way whatsoever. If you agree to participate, you may stop participating in the research at any time and tell me that you don't want to go continue. If you do this, there will be no penalties and you will not be prejudiced in any way.

\section{Confidentiality}

All identifying information will be kept in a locked file cabinet and will not be available to others and will be kept confidential to the extent possible by law. The records from your participation may be reviewed by people responsible for making sure that research is done properly, including members of the ethics committee at the Human Sciences Research Council. (All of these people are required to keep your identity confidential.) Otherwise, records that identify you, such as your patient number will be available only to people working on the study, unless you give permission for other people to see the records. We will not record your name anywhere and no 
one will be able to connect you to the answers you give. Your answers will be linked to a fictitious code number or a pseudonym (another name) and we will refer to you in this way in the data, any publication, report or other research output.

Some questions may be of a personal nature and you are free not to answer them if you do not wish to do so. We know that you cannot be absolutely certain about the answers to some of the questions. When it comes to answering questions there are no right and wrong answers. Your specific permission is requested in writing in the attached consent form. Your answers will be stored electronically in a secure environment and used for research or academic purposes now or at a later date in ways that will not reveal who you are. All future use of the stored data will be subject to further Research Ethics Committee review and approval.

\section{Risks/discomforts}

At the present time, we do not see any risk of harm from your participation. The risks associated with participation in this study are no greater than those encountered in daily life.

\section{Benefits}

There are no immediate benefits to you from participating in this study. However, this study will be extremely helpful to us in that we hope will promote understanding of the impact of diabetes on households and individuals and also determine the factors associated with adherence to medication and lifestyle modification in people with diabetes. If you would like to receive feedback on our study, we will record your phone number on a separate sheet of paper and can send you the results of the study when it is completed sometime after.

\section{Who to contact if you have been harmed or have any concerns}

This research has been approved by the HSRC Research Ethics Committee. If you have any complaints about ethical aspects of the research or feel that you have been harmed in any way by participating in this study, please call the HSRC's toll-free ethics hotline 0800212123 (when phoned from a landline from within South Africa) or contact the Human Sciences Research Council Research Ethics Committee Administrator, on Tel 0123022012 or e-mail research.ethics@hsrc.ac.za. You can also contact the University of Pretoria, Faculty of Health Sciences, (telephone numbers 0123563084 / 012356 3085). 
If you have concerns or questions about the research please feel free to contact the researchers - Ms Chipo Mutyambizi (CMutyambizi@hsrc.ac.za) and Prof Charles Hongoro (CHongoro@hsrc.ac.za) at the Human Sciences Research Council in Pretoria.

\section{CONSENT}

I hereby agree to participate in research on Costs and management of diabetes among South African adults. I understand that I am participating freely and without being forced in any way to do so. I also understand that I can stop participating at any point should I not want to continue and that this decision will not in any way affect me negatively. I understand that this is a research project whose purpose is not necessarily to benefit me personally in the immediate or short term. I understand that my participation will remain confidential.

Signature of participant

Date:

I understand that the information that I provide will be stored electronically and will be used for research purposes now or at a later stage. 


\section{Appendix F: Participant Quantitative Questionnaire}

Related to Chapter 3 and 5

number

\section{Preamble}

Taking part in this study is completely voluntarily and your confidentiality will be maintained. The questions asked tell us about your demographic profile, expenditures you make towards health care, your experiences with diabetic health care and also your adherence to diabetic medication and lifestyle modification.

The majority of the questions are relatively easy and straight to the point. The questionnaire will be administered by a field worker and will take between 30 to 45 minutes to complete

This questionnaire is to be administered to diabetic individuals on a fixed drug therapy, above the age of 21 .

INSTRUCTION TO INTERVIEWER

Record the answers by circling a response or by writing the answer in the space provided.

Please note that only one response is allowed per question unless another instruction is given.

Please note that coding categories should NOT be read to the participant unless another instruction

is given

\begin{tabular}{|l|l|}
\hline \multicolumn{2}{|c|}{ PARTICULARS } \\
\hline 1. Date & \\
\hline 2. Participant details (tick appropriate) & Patient above the age of 21 \\
\hline & Patient taking medication for diabetes \\
\hline 3. Interviewer details & \\
\hline 4. Health facility name & \\
\hline 5. Interview starting time & \\
\hline 6. Response code - Tick the appropriate one & $\begin{array}{l}1 \text { = Interview completed } \\
\text { 3 = Partly completed } \\
\text { 6 = Refusal by respondent } \\
7=\text { Other (Specify) }\end{array}$ \\
\hline
\end{tabular}




\section{SECTION A - PERSONAL INFORMATION}

\begin{tabular}{|c|c|c|c|c|}
\hline Q \# & Questions and Filters & Coding categories & Skip & Response \\
\hline \multirow{3}{*}{1} & \multirow{3}{*}{ What type of diabetes do you have } & 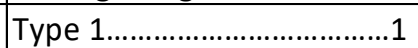 & & \\
\hline & & 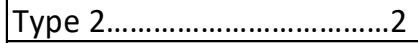 & & \\
\hline & & Unknown................................. & & \\
\hline \multirow{5}{*}{2} & \multirow{5}{*}{$\begin{array}{l}\text { Do you know what your HbA1c level was the last time it was } \\
\text { tested }\end{array}$} & 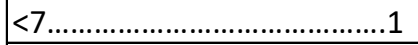 & & \\
\hline & & $7-8 \ldots \ldots \ldots \ldots \ldots \ldots \ldots \ldots \ldots \ldots \ldots \ldots \ldots . . .2$ & & \\
\hline & & 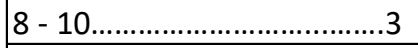 & & \\
\hline & & 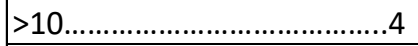 & & \\
\hline & & Don't know........................... & & \\
\hline 3 & $\begin{array}{l}\text { How old were you when a doctor or other health professional } \\
\text { first told you that you had high blood sugar or sugar diabetes }\end{array}$ & years & & \\
\hline 4 & $\begin{array}{l}\text { How old were you when a doctor or other health professional } \\
\text { first told you to take diabetes medication }\end{array}$ & years & & \\
\hline 5 & What is your age & years & & \\
\hline 6 & What is your height & mt & & \\
\hline 7 & What is your weight & kgs & & \\
\hline \multirow{4}{*}{8} & \multirow{4}{*}{ What diabetic medication are you currently taking } & Oral.......................................... & & \\
\hline & & 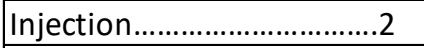 & & \\
\hline & & Oral and Injection...............3 & & \\
\hline & & Diet plan/exercise only.....4 & & \\
\hline \multirow{4}{*}{9} & \multirow{4}{*}{ How often do you take your ORAL medication in a day } & Once a day...........................1 & & \\
\hline & & Twice a day............................... & & \\
\hline & & Three times a day.................. & & \\
\hline & & Other (specify)......................4 & & \\
\hline \multirow{4}{*}{10} & & Once a day...........................1 & & \\
\hline & How often do vou take vour INIFCTION medication in a dav & Twice a day............................. & & \\
\hline & 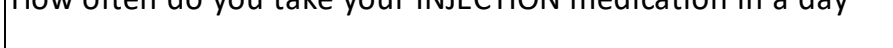 & Three times a day..................3 & & \\
\hline & & Other (specify)....................4 & & \\
\hline & $\begin{array}{l}\text { Has a doctor or nurse or health worker at a clinic or hospital } \\
\text { told you that you have any of the following conditions } \\
\text { (multiple response possible) }\end{array}$ & & & \\
\hline & (a) High blood pressure & Yes........................................ & & \\
\hline & 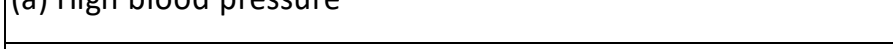 & 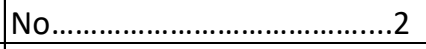 & & \\
\hline & (b) Denression & Yes......................................... & & \\
\hline & & 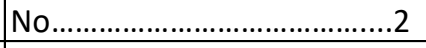 & & \\
\hline & (c) Heart disease & Yes.......................................1 & & \\
\hline 11 & (C) ireart unsease & 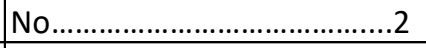 & & \\
\hline & (d) Retinonathv (Eve disease) & Yes............................................ & & \\
\hline & & 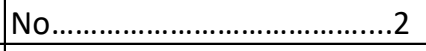 & & \\
\hline & (e) Neuronathy (nroblem with the nerves) & Yes............................................ & & \\
\hline & & 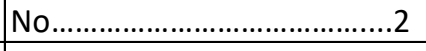 & & \\
\hline & (f) Kidnev disease & Yes......................................... & & \\
\hline & & 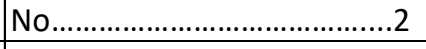 & & \\
\hline & (g) Other & Yes........................................ & & \\
\hline & 年 & 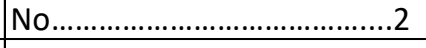 & & \\
\hline 12 & Has a doctor or nurse or health worker at a clinic or hospital & Yes............................................. & & \\
\hline & told you that you have had a stroke & 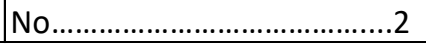 & & \\
\hline
\end{tabular}




\begin{tabular}{|c|c|c|c|}
\hline \multicolumn{4}{|c|}{ SECTION B - DEMOGRAPHIC AND HOUSEHOLD INFORMATION } \\
\hline \multirow{2}{*}{1} & \multirow{2}{*}{ What is your sex } & 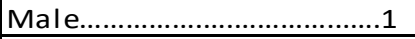 & \multirow{2}{*}{$\begin{array}{l}\text { If } 1 \mathrm{Go} \\
\text { to } 3\end{array}$} \\
\hline & & Female.................................... & \\
\hline \multirow{2}{*}{2} & \multirow{2}{*}{ Are you currently pregnant } & Yes ......................................1 & \\
\hline & & No & \\
\hline \multirow{5}{*}{3} & \multirow{5}{*}{ What is your race } & African.................................1 & \\
\hline & & White.....................................2 & \\
\hline & & Coloured................................. & \\
\hline & & Indian/Asian........................4 & \\
\hline & & Other(specify) .....................5 & \\
\hline \multirow{5}{*}{4} & \multirow{5}{*}{ Please indicate your marital status } & Single..................................1 & \\
\hline & & Married..................................2 & \\
\hline & & Divorced............................... & \\
\hline & & Widowed...............................4 & \\
\hline & & Co-habiting............................... & \\
\hline \multirow{2}{*}{5} & \multirow{2}{*}{$\begin{array}{l}\text { Has any close blood relative (father, mother, brother, sister } \\
\text { or child) ever been told by a doctor or other health } \\
\text { professional that they have diabetes }\end{array}$} & Yes ……........................................ & \\
\hline & & No......................................... & \\
\hline \multirow{2}{*}{6} & \multirow{2}{*}{ Do you have any children } & \multirow{2}{*}{ 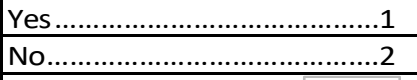 } & \multirow{2}{*}{$\begin{array}{l}\text { If } 2 \mathrm{Go} \\
\text { to } 8\end{array}$} \\
\hline & & & \\
\hline 7 & How many (alive) children do you have? & \multirow{2}{*}{ 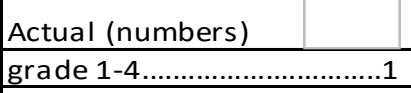 } & \\
\hline \multirow{6}{*}{8} & \multirow{6}{*}{ Please indicate your highest education level } & & \\
\hline & & \multirow{2}{*}{ 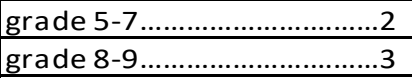 } & \\
\hline & & & \\
\hline & & 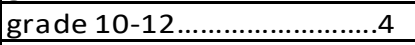 & \\
\hline & & College.....................................5 & \\
\hline & & 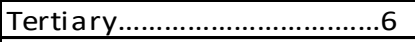 & \\
\hline \multirow{16}{*}{9} & \multirow{16}{*}{ How would you describe your present employment situation } & 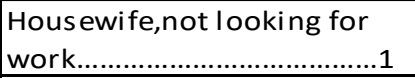 & \\
\hline & & $\begin{array}{l}\text { Hous ewife,looking for } \\
\text { work.................................2 }\end{array}$ & \\
\hline & & 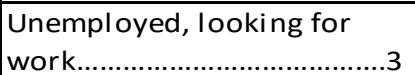 & \\
\hline & & Unemployed, not looking for & \\
\hline & & work ............................... & \\
\hline & & $\begin{array}{l}\text { Work in informal sector, not } \\
\text { looking for permanent } \\
\text { work................................. }\end{array}$ & \\
\hline & & $\begin{array}{l}\text { Work in informal sector, } \\
\text { looking for permanent } \\
\text { work................................... }\end{array}$ & \\
\hline & & $\begin{array}{l}\text { Sick/ disabled and unable to } \\
\text { work }\end{array}$ & \\
\hline & & Student/pupil/learner..........8 & \\
\hline & & $\begin{array}{l}\text { Self employed - Full time (40 } \\
\text { hrs or more per week).........9 }\end{array}$ & \\
\hline & & 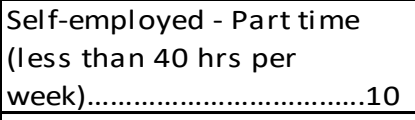 & \\
\hline & & $\begin{array}{l}\text { Employed - Part time (less } \\
\text { than } 40 \text { hrs per } \\
\text { week) }\end{array}$ & \\
\hline & & $\begin{array}{l}\text { Employed - Full time (40 hrs } \\
\text { or more per week).............12 }\end{array}$ & \\
\hline & & \begin{tabular}{|l} 
Employed (Seasonal \\
Work)...........................13
\end{tabular} & \\
\hline & & Reti red..................................14 & \\
\hline & & 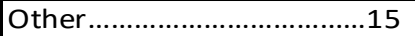 & \\
\hline
\end{tabular}




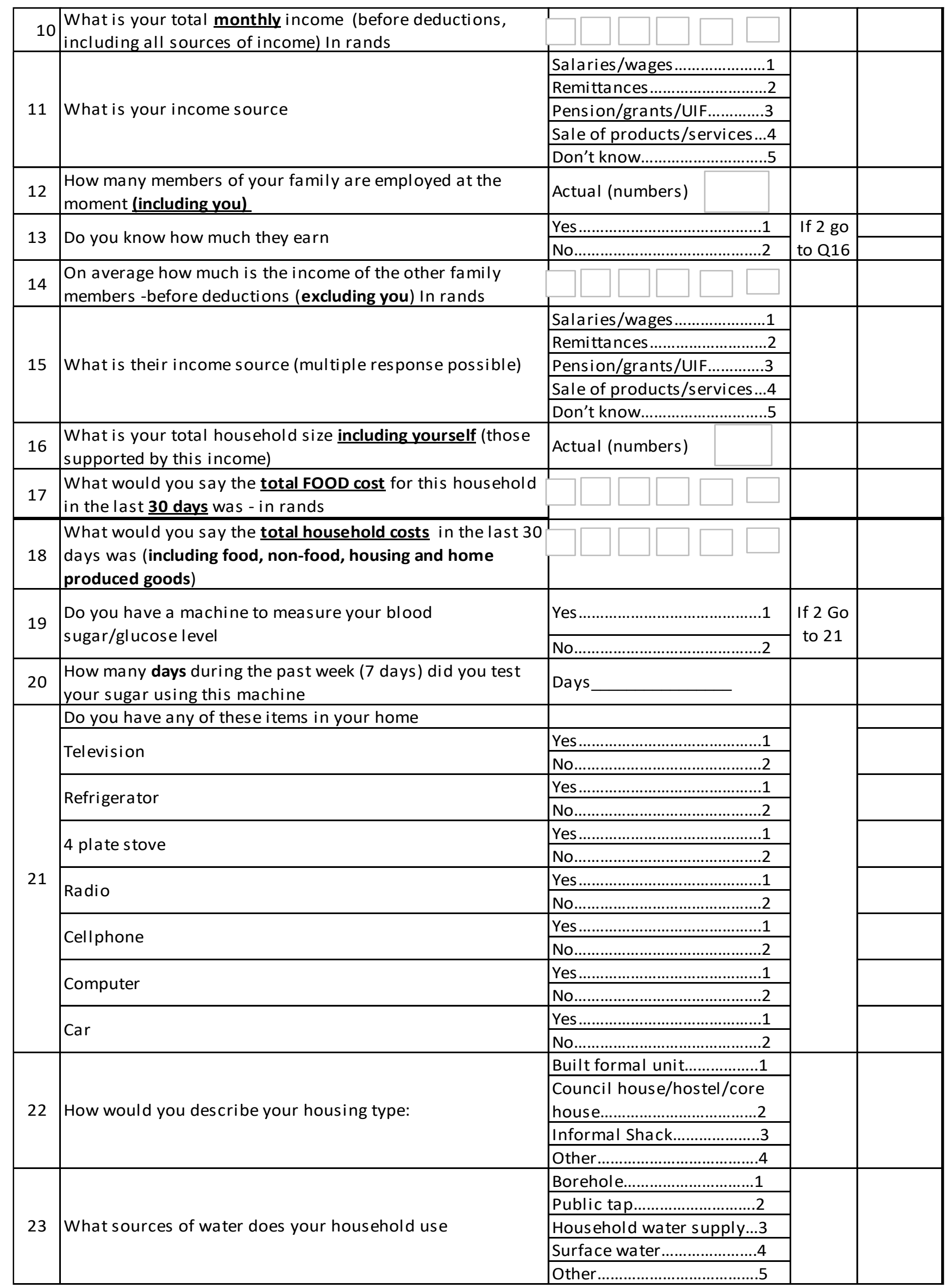




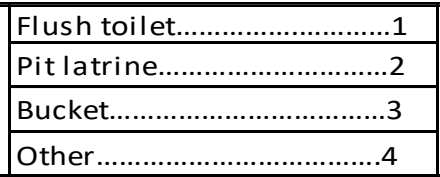

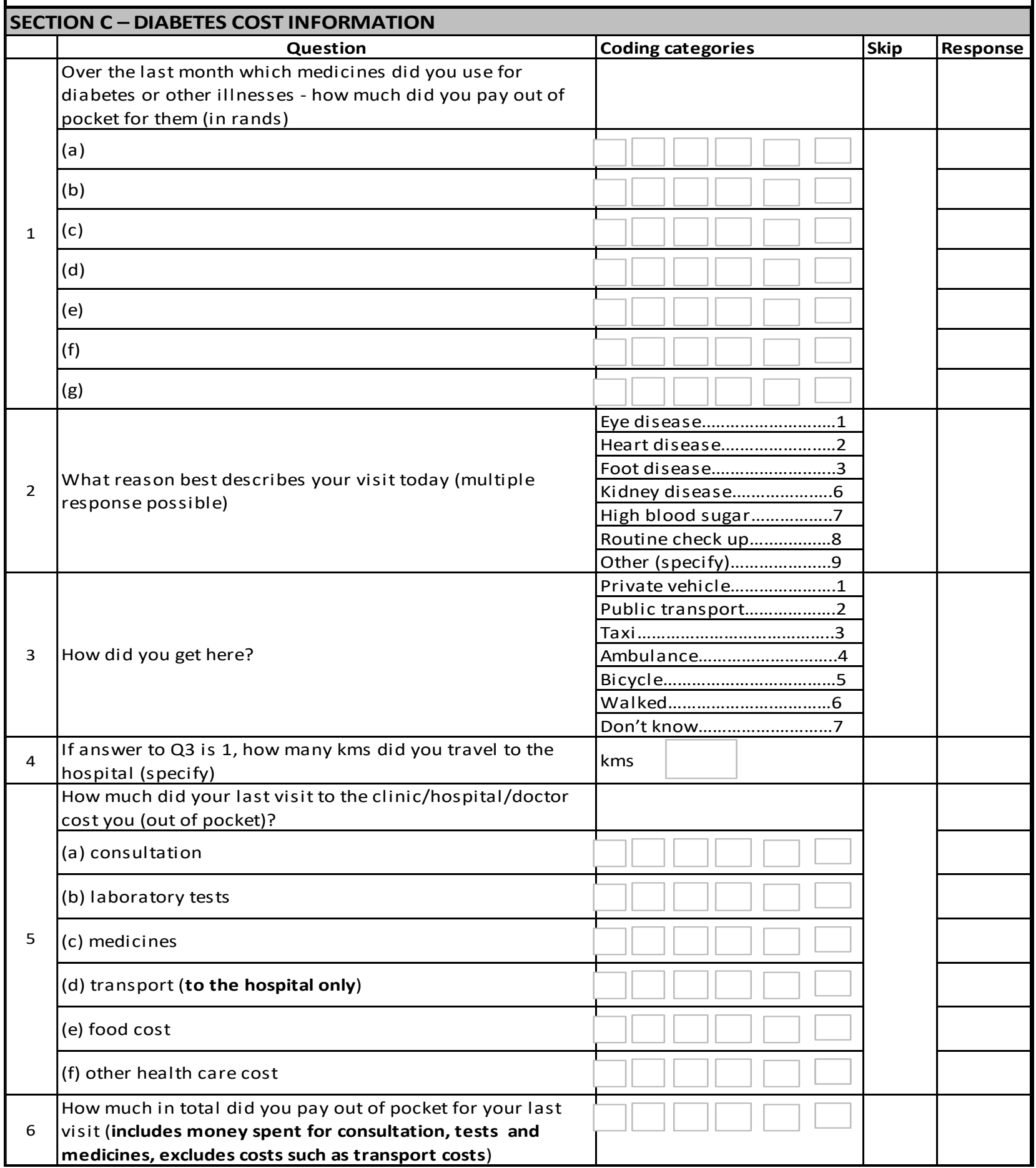




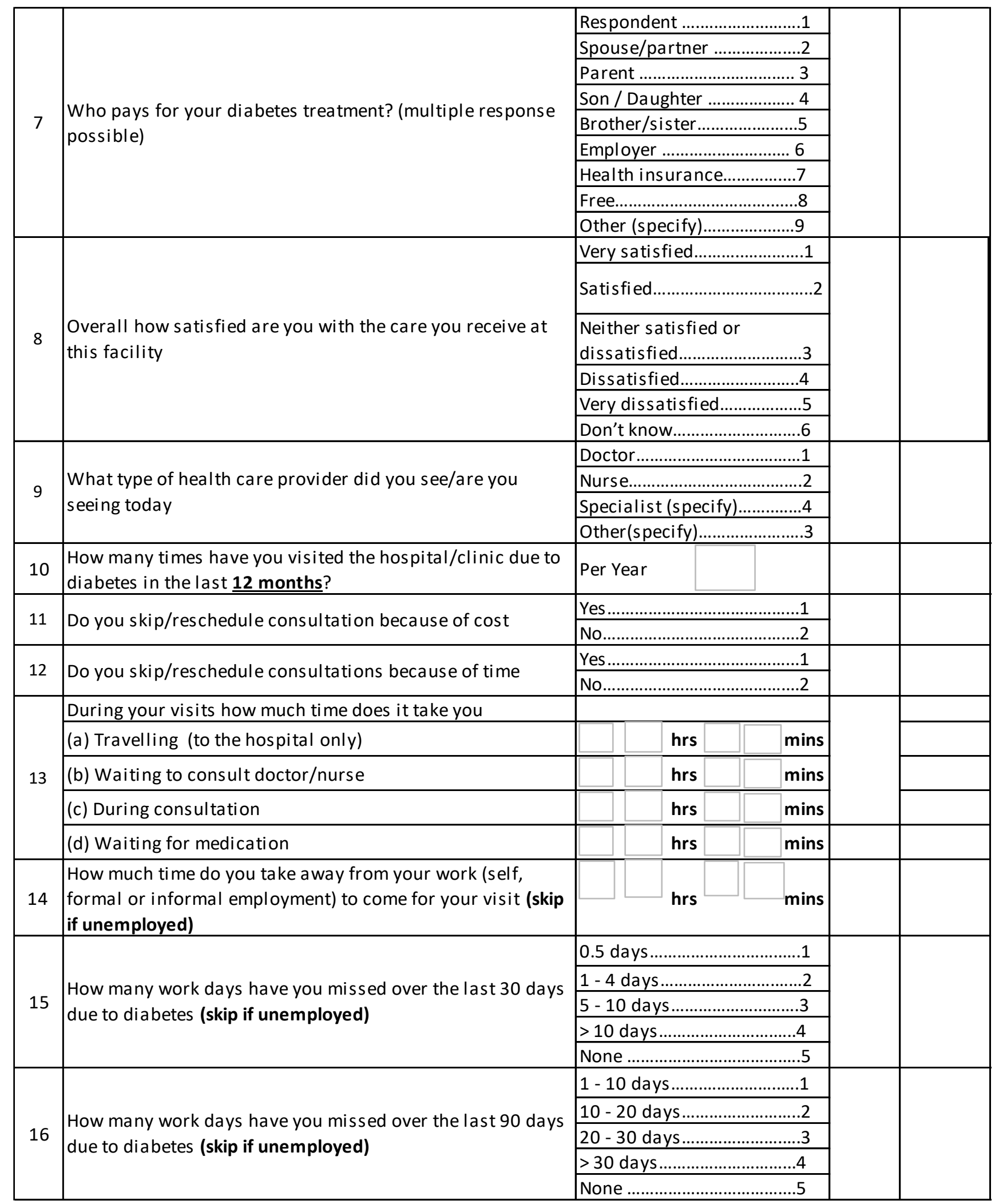




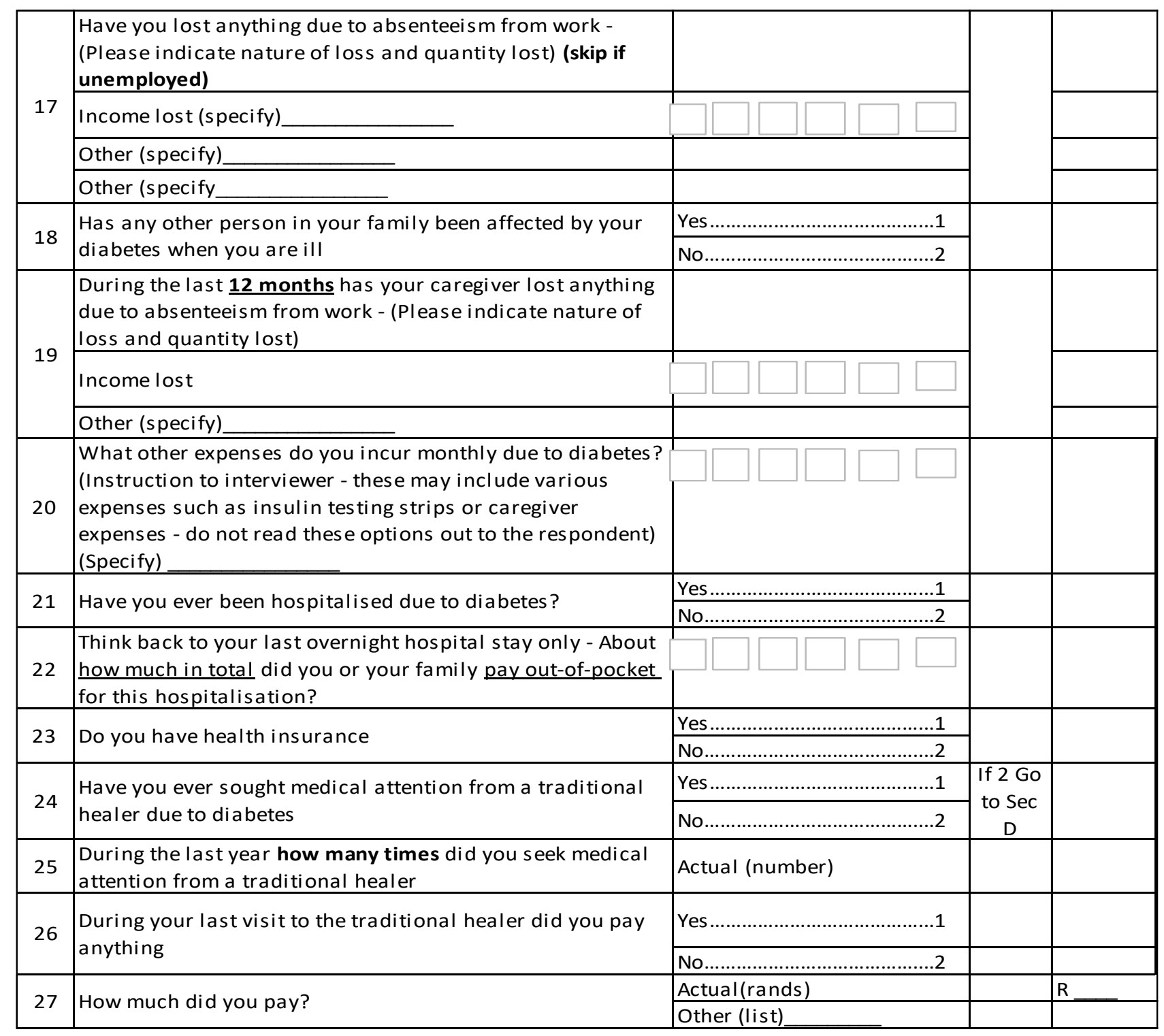

\begin{tabular}{|c|c|c|c|}
\hline SEC & ION D - ADHERENCE TO MEDICATION & & \\
\hline & & A lot of benefits.................... & \\
\hline 1 & tablets/insulin) & Some benefits ......................2 & \\
\hline & & No Benefits............................3 & \\
\hline & & Sometimes ...........................1 & \\
\hline 2 & medication at this hosnital slinis & Al ways ....................................... & \\
\hline & & Other ...................................... & \\
\hline 3 & Have you ever experienced side effects due to diabetic & Yes .......................................... & \\
\hline 3 & medication & No....................................... & \\
\hline 4 & J Have vou ever received diabetic education & Yes......................................1 & \\
\hline 4 & 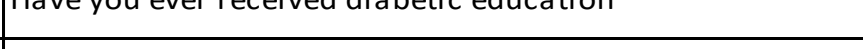 & No.........................................2 & \\
\hline 5 & Dovou sometimes forget to take vour medication & Yes .......................................1 & \\
\hline כ & Do you somentmes rorget to lake your mearcation & No............................................ & \\
\hline & Are you at times careless about taking your medication (i.e & Yes.........................................1 & \\
\hline 6 & not taking sufficient care or taking it at the wrong time) & No.................................... & 2 to \\
\hline 7 & When you felt better, did you sometimes stop taking your & Yes ...................................... & \\
\hline$r^{\prime}$ & medication & No........................................... & \\
\hline 8 & Sometimes, if you felt worse when you took your medicine & Yes..........................................1 & \\
\hline & did you stop taking it & No & \\
\hline
\end{tabular}




\begin{tabular}{|c|c|c|}
\hline \multirow{8}{*}{9} & $\begin{array}{l}\text { Now I would like you to recall if you have missed any doses } \\
\text { of medication on a day by day basis over the last } 7 \text { days, } \\
\text { how many doses did you miss on - }\end{array}$ & \\
\hline & Day 7 (day before interview) & Actual (numbers) \\
\hline & Day 6 & Actual (numbers) \\
\hline & Day 5 & Actual (numbers) \\
\hline & Day 4 & Actual (numbers) \\
\hline & Day 3 & Actual (numbers) \\
\hline & Day 2 & Actual (numbers) \\
\hline & Day 1 & Actual (numbers) \\
\hline \multirow{7}{*}{10} & \multirow{7}{*}{$\begin{array}{l}\text { What reason best describes your reason for poor adherence } \\
\text { to medication (multiple responses possible) (Instruction to } \\
\text { interviewer - only ask to individuals who respondent yes to } \\
\text { any of Q5-9) }\end{array}$} & 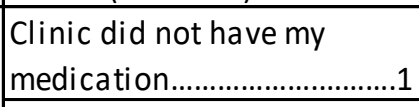 \\
\hline & & 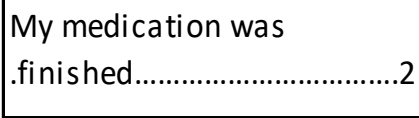 \\
\hline & & $\begin{array}{l}\text { My medication makes me } \\
\text { worse................................... }\end{array}$ \\
\hline & & I forgot................................. \\
\hline & & I was too busy....................... \\
\hline & & \begin{tabular}{|l|} 
I did not go to the clinic to \\
collect medication..............6
\end{tabular} \\
\hline & & Other ................................ \\
\hline
\end{tabular}

\section{SECTION E - ADHERENCE TO EXERCISE}

\begin{tabular}{c|l}
\hline 1 & Have you ever received any detailed instruction regarding
\end{tabular}

1 exercise from any healthcare provider

2 Do you think that exercise helps to control and maintain

2 blood glucose levels

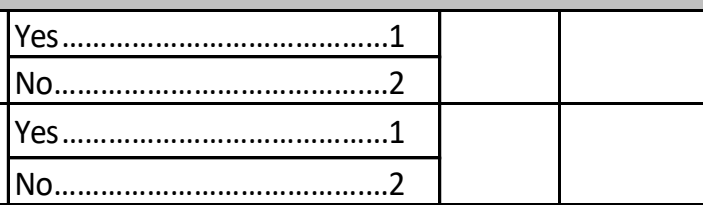

\begin{tabular}{|c|c|c|c|}
\hline \multicolumn{4}{|c|}{ During the past week, how much time (for the entire week) did you spend doing each of the following } \\
\hline \multirow{5}{*}{3} & \multirow{5}{*}{ Bicycling (including stationary exercise bikes) } & None.........................................1 & \\
\hline & & $<30 \mathrm{mins} /$ week........................2 & \\
\hline & & 30-60mins/week....................3 & \\
\hline & & 1-2 hours/week......................4 & \\
\hline & & >2 hours/week.......................5 & \\
\hline \multirow{5}{*}{4} & \multirow{5}{*}{ Brisk walking/Jogging } & None......................................1 & \\
\hline & & $<30$ mins/week........................2 & \\
\hline & & 30-60mins/week....................3 & \\
\hline & & 1-2 hours/week....................4 & \\
\hline & & $>2$ hours/week.......................5 & \\
\hline \multirow{5}{*}{5} & \multirow{5}{*}{ Sport activates (e.g tennis, Swimming, soccer) } & None.........................................1 & \\
\hline & & $<30$ mins/week.......................2 & \\
\hline & & 30-60mins/week...................3 & \\
\hline & & 1-2 hours/week.....................4 & \\
\hline & & $>2$ hours/week......................5 & \\
\hline \multirow{5}{*}{6} & \multirow{5}{*}{ Stretching or strengthening exercises (e.g heavy lifting etc) } & None...................................... & \\
\hline & & $<30$ mins/week.........................2 & \\
\hline & & 30-60mins/week....................3 & \\
\hline & & 1-2 hours/week....................4 & \\
\hline & & $>2$ hours/week.......................5 & \\
\hline
\end{tabular}




\begin{tabular}{|c|c|c|c|c|}
\hline \multirow{5}{*}{7} & \multirow{5}{*}{ Aerobic exercise (e.g rowing, stepper etc) } & None.......................................1 & & \\
\hline & & $<30$ mins/week.........................2 & & \\
\hline & & 30-60mins/week....................3 & & \\
\hline & & 1-2 hours/week......................4 & & \\
\hline & & $>2$ hours/week......................... & & \\
\hline \multirow{5}{*}{8} & \multirow{5}{*}{ Other (Specify) } & None........................................ & & \\
\hline & & $<30$ mins/week.......................2 & & \\
\hline & & 30-60mins/week....................3 & & \\
\hline & & 1-2 hours/week....................4 & & \\
\hline & & $>2$ hours/week........................5 & & \\
\hline \multirow{6}{*}{9} & \multirow{6}{*}{$\begin{array}{l}\text { What reason best describes your reason for non-adherence } \\
\text { to exercise (multiple responses possible) }\end{array}$} & $\begin{array}{l}\text { I do not believe it helps } \\
\text { control blood glucose } \\
\text { level s.................................... }\end{array}$ & & \\
\hline & & I am too busy .......................2 & & \\
\hline & & $\begin{array}{l}\text { I am too sick to } \\
\text { exercise.............................. } 3\end{array}$ & & \\
\hline & & $\begin{array}{l}\text { I feel worse after } \\
\text { exercise.............................4 }\end{array}$ & & \\
\hline & & $\begin{array}{l}\text { I a } \mathrm{m} \text { too old to } \\
\text { exercise..............................5 }\end{array}$ & & \\
\hline & & Other ...................................6 & & \\
\hline \multirow{5}{*}{10} & \multirow{5}{*}{ Do you have any difficulty in day-to-day work } & 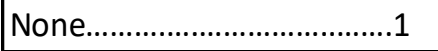 & & \\
\hline & & 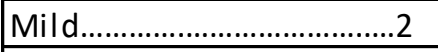 & & \\
\hline & & Moderate...............................3 & & \\
\hline & & Severe................................... & & \\
\hline & & 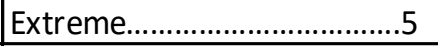 & & \\
\hline \multicolumn{5}{|c|}{ SECTION F - ADHERENCE TO DIET } \\
\hline \multirow{2}{*}{1} & \multirow{2}{*}{$\begin{array}{l}\text { Have you ever received any detailed instruction regarding } \\
\text { healthy dietary habits from any healthcare provider }\end{array}$} & 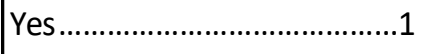 & & \\
\hline & & 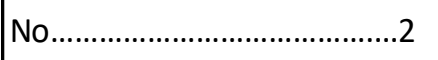 & & \\
\hline \multirow{3}{*}{2} & \multirow{3}{*}{$\begin{array}{l}\text { Do you think that healthy dietary intake helps to control } \\
\text { and maintain blood glucose levels }\end{array}$} & Yes.......................................... & & \\
\hline & & 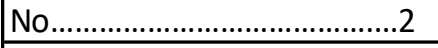 & & \\
\hline & & Don't know.............................. & & \\
\hline \multirow{2}{*}{3} & \multirow{2}{*}{$\begin{array}{l}\text { Do you adhere to the healthy dietary habits that were } \\
\text { recommended to you by a dietician/doctor }\end{array}$} & Yes ............................................. & If $1 \mathrm{Go}$ & \\
\hline & & 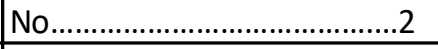 & to 5 & \\
\hline \multirow{5}{*}{4} & \multirow{5}{*}{$\begin{array}{l}\text { What reason best describes your reason for non-adherence } \\
\text { to the recommended dietary habits }\end{array}$} & Too busy ..................................... & & \\
\hline & & 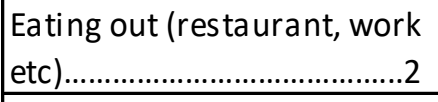 & & \\
\hline & & $\begin{array}{l}\text { Financial constraints } \\
\text { (dietary recommendations } \\
\text { too expensive).......................3 }\end{array}$ & & \\
\hline & & Poor self control .................4 & & \\
\hline & & Other (specify)......................5 & & \\
\hline
\end{tabular}




\begin{tabular}{|c|c|c|c|c|}
\hline \multicolumn{5}{|c|}{ During the past seven days did you eat the following food } \\
\hline \multirow{4}{*}{5} & \multirow{4}{*}{$\begin{array}{l}\text { Fresh fruit (all types of fruit excluding fruit juices and dried } \\
\text { fruit }\end{array}$} & None.........................................1 & & \\
\hline & & Everyday.................................. & & \\
\hline & & $1-3$ times last week..........3 & & \\
\hline & & 4-6 times last week............4 & & \\
\hline \multirow{4}{*}{6} & \multirow{4}{*}{ Dark green leafy or yellow vegetables } & None......................................1 & & \\
\hline & & 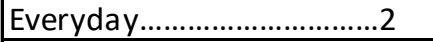 & & \\
\hline & & $1-3$ times last week...........3 & & \\
\hline & & 4-6 times last week............4 & & \\
\hline \multirow{4}{*}{7} & \multirow{4}{*}{$\begin{array}{l}\text { Other vegetables (e.g cabbage, tomatoes, excluding } \\
\text { potatoes)? }\end{array}$} & None........................................ & & \\
\hline & & Everyday.................................. & & \\
\hline & & $1-3$ times last week..........3 & & \\
\hline & & 4-6 times last week............4 & & \\
\hline \multirow{4}{*}{8} & \multirow{4}{*}{$\begin{array}{l}\text { Processed meat, e.g. sausages, polony, cold cuts, Viennas, } \\
\text { Frankfurters, Russians, salami? }\end{array}$} & None....................................... & & \\
\hline & & Everyday.................................. & & \\
\hline & & 1 - 3 times last week..........3 & & \\
\hline & & 4-6 times last week............4 & & \\
\hline \multirow{4}{*}{9} & \multirow{4}{*}{$\begin{array}{l}\text { Food deep fried in oil/fat, e.g. fish, fries/chips, vetkoek, } \\
\text { samoosas, doughnuts, vetkoek? }\end{array}$} & None....................................... & & \\
\hline & & Everyday................................. & & \\
\hline & & 1 - 3 times last week..........3 & & \\
\hline & & 4-6 times last week.............4 & & \\
\hline \multirow{4}{*}{10} & \multirow{4}{*}{$\begin{array}{l}\text { Salty foods, e.g. salted nuts, biltong, dried sausage, dried } \\
\text { salted fish? }\end{array}$} & None...................................... & & \\
\hline & & Everyday................................ & & \\
\hline & & $1-3$ times last week..........3 & & \\
\hline & & 4-6 times last week............4 & & \\
\hline \multirow{4}{*}{11} & \multirow{4}{*}{ Sugar added beverages and / sweetened fruit juice? } & None...................................... & & \\
\hline & & Everyday................................. & & \\
\hline & & $1-3$ times last week..........3 & & \\
\hline & & 4-6 times last week............4 & & \\
\hline \multicolumn{5}{|c|}{ FOOD SECURITY } \\
\hline \multirow{2}{*}{12} & \multirow{2}{*}{ Does your household ever run out of money to buy food? } & Yes.........................................1 & If $2 \mathrm{Go}$ & \\
\hline & & No............................................ & to 15 & \\
\hline \multirow{2}{*}{13} & \multirow{2}{*}{ Has it happened in the past 30 days? } & Yes........................................... & & \\
\hline & & 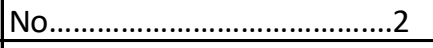 & & \\
\hline \multirow{2}{*}{14} & \multirow{2}{*}{ Has it happened 5 or more days in the past 30 days? } & Yes........................................... & & \\
\hline & & No............................................. & & \\
\hline \multicolumn{5}{|c|}{ DIETARY PRACTICES } \\
\hline & & Brown sugar........................1 & & \\
\hline & & White sugar...........................2 & & \\
\hline 15 & Which sweeteners do you use (multinle resnonse nossible)? & Honey....................................... & & \\
\hline ال & 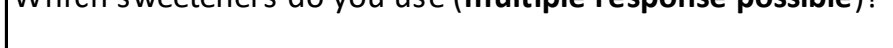 & Artificial Sweeteners...........4 & & \\
\hline & & None & & \\
\hline & & Other (specify)......................6 & & \\
\hline & & Diet soft drinks.....................1 & & \\
\hline 16 & arsible) & Regular soft drinks...............2 & & \\
\hline & & 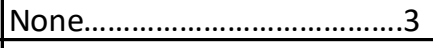 & & \\
\hline 17 & Is your shopping list of food different from the list of food & Yes........................................... & & \\
\hline & & 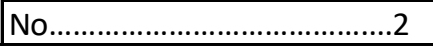 & & \\
\hline
\end{tabular}




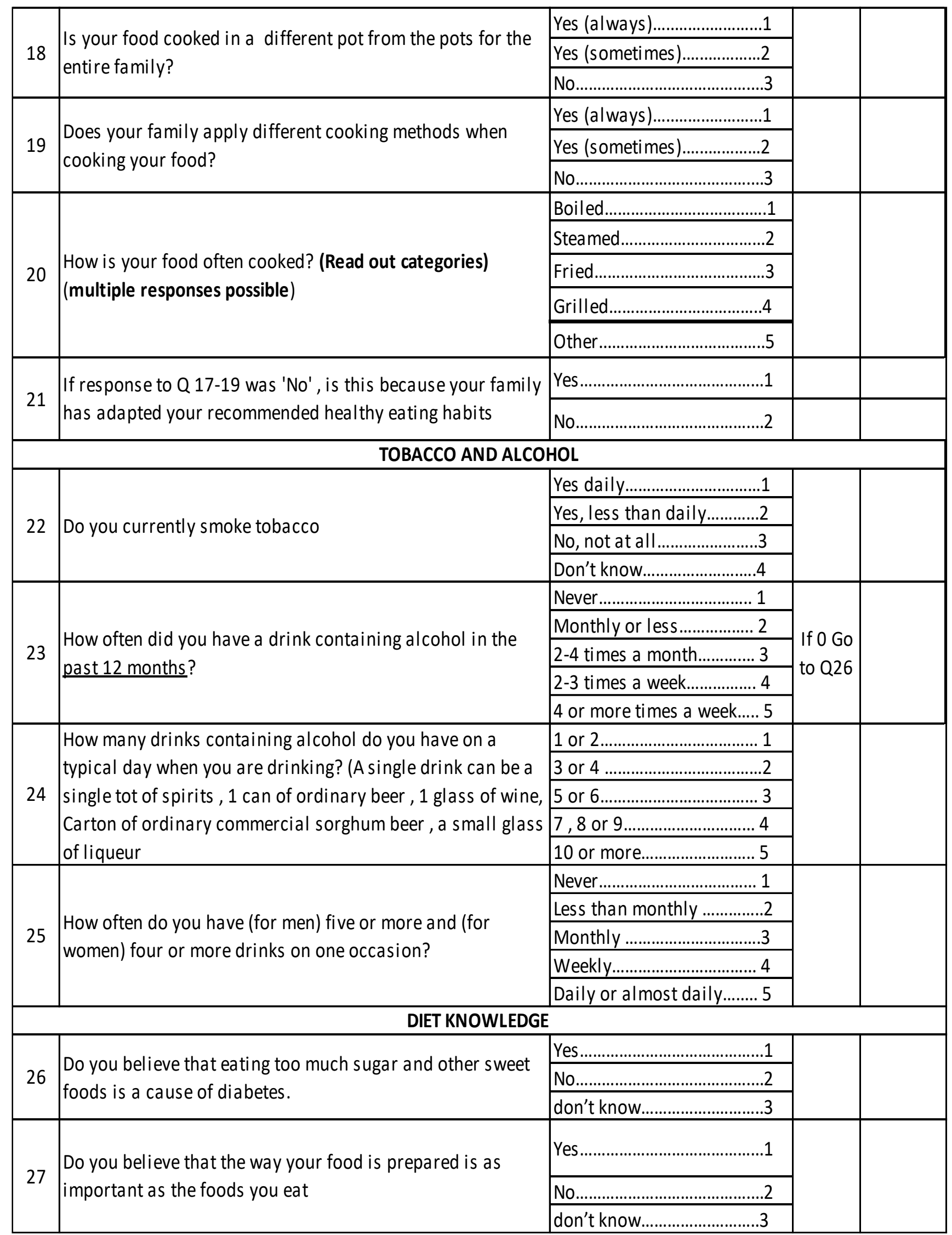


SECTION G

Please describe the foods (meals and snacks and drinks) you ate yesterday during the day and night

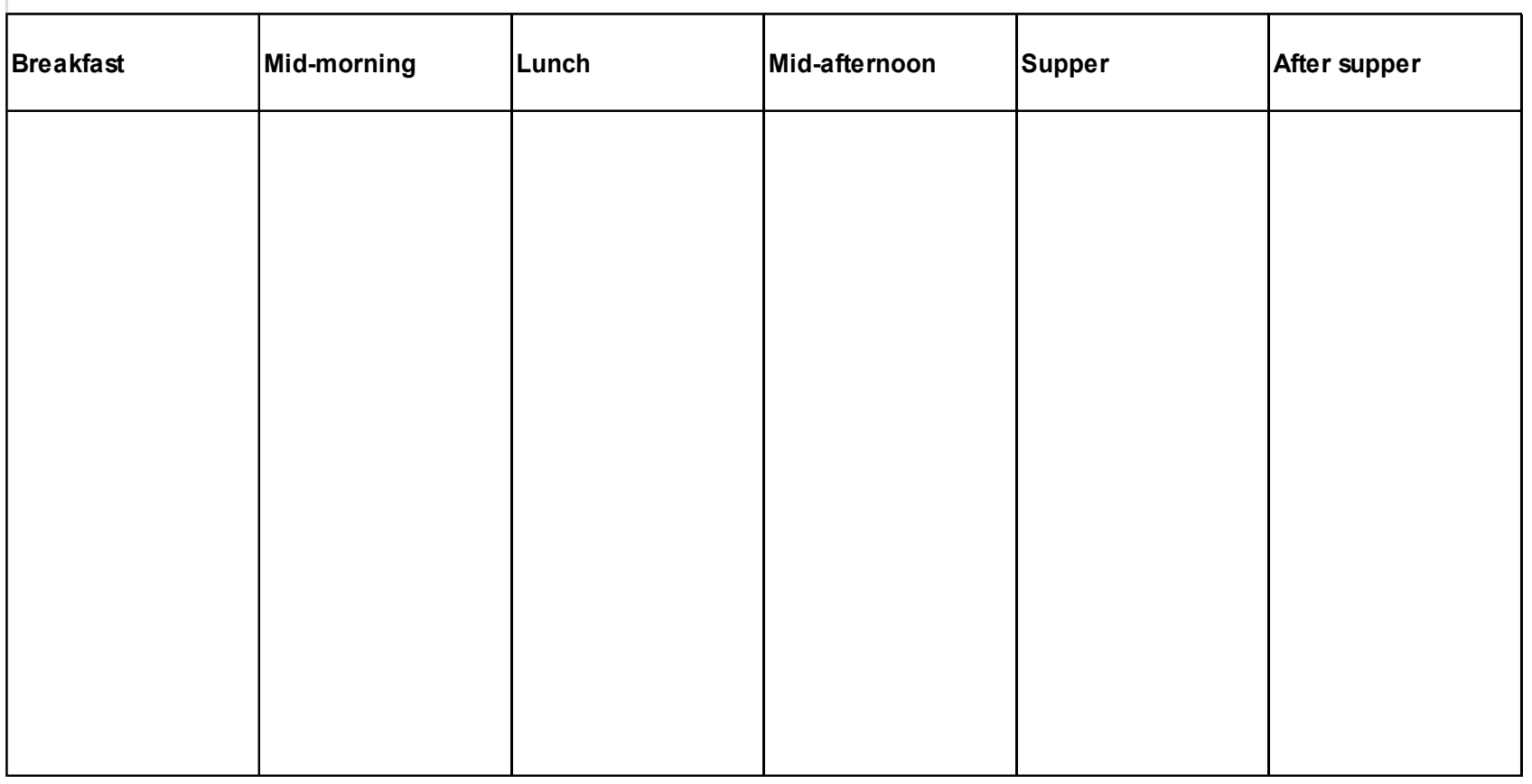

\begin{tabular}{l|l|l}
\hline NO. & QUESTIONS AND FLTTERS & CODING CATEGORIES \\
\hline 1 & $\begin{array}{l}\text { Did you eat anything (meal or snack) OUTSIDE your home } \\
\text { yesterday? }\end{array}$ & Yes.......1 \\
\hline 2 & $\begin{array}{l}\text { Was yesterday a celebration or feast day where you ate special } \\
\text { foods or where you ate more, or less than usual? }\end{array}$ & $\begin{array}{l}\text { Yes........1 } \\
\text { No.........2 }\end{array}$ \\
\hline
\end{tabular}




\section{INSTRUCTION TO INTERVIEWER}

SECTION G

Group

3 Cereals

4 White roots and tubers

5 Yellow/orange vegetables

6 Dark-green leaves

\begin{tabular}{|l|l}
\hline 7 & Vegetables other than dark- green leafy and yellow/orange
\end{tabular}

8 Yellow / orange fruits

9 Fruit other than yellow / orange fleshed

10 Organ meat (offal)

11 Meat and poultry (flesh meats)

12 Eggs

13 Fish and seafood

14 Legumes, nuts and seeds

15 Milk and milk products

16 Fats and oils

17 Sugars and sweets

18 Spices and condiments

19 Drinks

20 Drinks

21 Drinks

21 Drinks
When the interview with the participant is completed, the fieldworker must select the individual food items listed in Section D-1 and link them to the appropriate food groups listed in Section D-2.

At the end of the day, the team leader will then double check that the linking has been done correctly

Foods

Code

Corn/maize/samp, rice, wheat, sorghum, porridge, phutu, bread, pasta, breakfast cereals, oats, Mabella, Morvite, Potato, white sweet potato

Carrot, butternut, pumpkin, orange-fleshed sweet potato

Spinach, imifino, morogo

Yes.........1 No...........2

Yes........1 No...........2

Yes.........1 No...........2

Beetroot, brinjals, broccoli, brussels sprouts, cabbage, cauliflower, gem squash, green beans, onion, peas, tomato, Yes........1 No..........2

Apricot, mango, pawpaw, sweet melon, yellow flesh peach, yellow flesh plums, $100 \%$ fruit juice made from these Apple, avocado, banana, berries, fig, granadilla, grape, grapefruit, guava, lemon, litchi, maroela, melon, orange,

Liver, kidney, heart, spleen, lungs, chicken giblets, malomogudo (offal), intestines

Beef, goat, lamb, mutton, pork, venison, game, chicken, birds, ostrich, insects, mopani worms, chicken head/feet, Any type of egg

Fresh, frozen fish or canned fish (sardines, pilchards, tuna), dried fish, shellfish

Dried beans, dried peas, lentils, nuts, peanuts, seeds (or foods made from these e.g. peanut butter)

Milk, sour milk, cheese, yogurt, custard, or any other milk products, or any drinks made with milk eg. cocoa

Oils, fats, margarine or butter added to foods or used for cooking

Sugar, sweets, chocolates, cake and sweetened biscuits, honey, jam, sugar sweetened drinks e.g. cold drinks,

Spices (salt, pepper, etc), condiments (e.g. chutney, tomato sauce)

Coffee, tea

Alcoholic drinks

Cold drinks (except diet cold drinks) and sweetened beverages

Cold drinks-diet cold drinks

Yes........1 No...........2

Yes........1 No..........2

Yes........1 No...........2

Yes........1 No..........2

Yes........1 No..........2

Yes........1 No..........2

Yes........1 No..........2

Yes........1 No..........2

Yes.........1 No...........2

Yes........1 No..........2

Yes........1 No..........2

Yes........1 No..........2

Yes........1 No..........2

Yes........1 No...........2

Yes........1 No...........2 


\section{Appendix G: Participant consent Form-Qualitative data}

Related to Chapter 6

Title of study: Costs and management of diabetes among South African adults

\section{Who we are}

Hello, my name is I am working for the Human Sciences Research Council.

\section{What we are doing}

We are conducting research on the Costs and management of diabetes among South African adults. We are conducting a study to establish the impact of diabetes on households and individuals and also determine the factors associated with adherence to medication and lifestyle modification.

\section{Your participation}

We are asking you whether you will allow us to interview with you about your experiences with diabetic health care and adherence to diabetic medication and lifestyle modification. If you agree, we will ask you to participate in one interview.

Please understand that your participation is voluntary and you are not being forced to take part in this study. The choice of whether to participate or not, is yours alone. If you choose not to take part, you will not be affected in any way whatsoever. If you agree to participate, you may stop participating in the research at any time and tell me that you don't want to go continue. If you do this, there will be no penalties and you will not be prejudiced in any way.

We would also like to seek your permission to extract information regarding your height, weight, presence of comorbidities, type of diabetes, glycaemic control levels, medication regime and date of diagnosis from your medical records.

\section{Confidentiality}

All identifying information will be kept in a locked file cabinet and will not be available to others and will be kept confidential to the extent possible by law. The records from your participation may be reviewed by people responsible for making sure that research is done properly, including 
members of the ethics committee at the Human Sciences Research Council. (All of these people are required to keep your identity confidential.) Otherwise, records that identify you, such as your patient number will be available only to people working on the study, unless you give permission for other people to see the records.

We are asking you to give us permission to tape-record the interview so that we can accurately record what is said. Your answers will be stored electronically in a secure environment and used for research or academic purposes now or at a later date in ways that will not reveal who you are. All future use of the stored data will be subject to further Research Ethics Committee review and approval.

We will not record your name anywhere and no one will be able to connect you to the answers you give. Your answers will be linked to a fictitious code number or a pseudonym (another name) and we will refer to you in this way in the data, any publication, report or other research output.

\section{Risks/discomforts}

At the present time, we do not see any risk of harm from your participation. The risks associated with participation in this study are no greater than those encountered in daily life.

\section{Benefits}

There are no immediate benefits to you from participating in this study. However, this study will be extremely helpful to us in that we hope will promote understanding of the impact of diabetes on households and individuals and also determine the factors associated with adherence to medication and lifestyle modification in diabetic individuals

If you would like to receive feedback on our study, we will record your phone number on a separate sheet of paper and can send you the results of the study when it is completed sometime after.

\section{Who to contact if you have been harmed or have any concerns}

This research has been approved by the Human Science Research Council Research Ethics Committee. If you have any complaints about ethical aspects of the research or feel that you have 
been harmed in any way by participating in this study, please call the HSRC's toll-free ethics hotline 0800212123 (when phoned from a landline from within South Africa) or contact the Human Sciences Research Council Research Ethics Committee Administrator, on Tel 012302 2012 or e-mail research.ethics@hsrc.ac.za.

If you have concerns or questions about the research please feel free to contact the researchers - Ms Chipo Mutyambizi (Email - CMutyambizi@hsrc.ac.za; Tel - 012302 2340) and Prof Charles Hongoro (Email - CHongoro@hsrc.ac.za) at the Human Sciences Research Council in Pretoria.

\section{CONSENT}

I hereby agree to participate in research on Costs and management of diabetes among South African adults. I understand that I am participating freely and without being forced in any way to do so. I also understand that I can stop participating at any point should I not want to continue and that this decision will not in any way affect me negatively. I understand that this is a research project whose purpose is not necessarily to benefit me personally in the immediate or short term. I understand that my participation will remain confidential.

Signature of participant

Date:

I hereby agree to the audio tape-recording of the discussions during my participation in the interview

Signature of participant

Date:

I understand that the information that I provide will be stored electronically and will be used for research purposes now or at a later stage. 
Date: 


\section{Appendix H: Interview guide}

Related to Chapter 6

\section{Questionnaire number}

COSTS AND MANAGEMENT OF DIABETES AMONG SOUTH AFRICAN ADULTS

Qualitative data collection - Interview protocol

INSTRUCTION TO INTERVIEWER

SECTION A: Record the answers in the blank space provided.

SECTION B: This is an in-depth interview. Use a voice recorder and note taker this section.

\section{Preamble}

Now I would like you to discuss your experiences with diabetes care management.

I encourage you to respond freely and openly and say as much as you would like. I will be voice recording the discussion because I would like to capture all your comments and remarks. Please be assured that no one outside of this room will have access to my recordings.

The majority of the questions are relatively easy and straight to the point. Please answer all questions honestly.

Is it ok to audiotape this interview?

\begin{tabular}{|l|l|}
\hline \multicolumn{2}{|c|}{ PARTICULARS } \\
\hline 1. Date & \\
\hline 2. Patient Study number & \\
\hline 3. Interviewer details & \\
\hline 3. Health facility name & \\
\hline 5. Interview starting time & $1=$ Interview completed \\
\hline 6. Response code - Tick the appropriate one & $\begin{array}{l}\text { 3 = Partly completed } \\
6=\text { Refusal by respondent } \\
7=\text { Other (Specify) }\end{array}$ \\
\hline 7. Consent form completed (tick) & \\
\hline
\end{tabular}




\begin{tabular}{|c|c|c|c|}
\hline \multicolumn{4}{|c|}{ SECTION A - PERSONAL INFORMATION } \\
\hline Q \# & Questions and Filters & Coding categories & Response \\
\hline \multirow{3}{*}{1} & \multirow{3}{*}{ What type of diabetes do you have } & 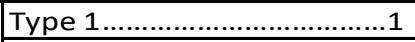 & \\
\hline & & 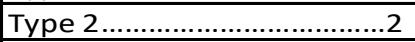 & \\
\hline & & Unknown............................... & \\
\hline 2 & $\begin{array}{l}\text { How old were you when a doctor or other health } \\
\text { professional first told you that you had high blood sugar or } \\
\text { sugar diabetes }\end{array}$ & (years) & \\
\hline 3 & What is your age & (years) & \\
\hline 4 & What is your height & metres, centi metres & \\
\hline 5 & What is your weight & kgs & \\
\hline \multirow{4}{*}{6} & \multirow{4}{*}{ What diabetic medication are you currently taking } & Oral.................................. & \\
\hline & & Injection............................ & \\
\hline & & Oral and Injection..............3 & \\
\hline & & Diet plan/exercise only.....4 & \\
\hline \multirow{4}{*}{7} & \multirow{4}{*}{ How often do you take your ORAL medication in a day } & Once a day.........................1 & \\
\hline & & Twice a day.......................... & \\
\hline & & Three times a day...............3 & \\
\hline & & Other (specify) ...................4 & \\
\hline \multirow{4}{*}{9} & \multirow{4}{*}{ How often do you take your INJECTION medication in a day } & Once a day..........................1 & \\
\hline & & Twice a day.........................2 & \\
\hline & & Three times a day.................3 & \\
\hline & & Other (s pecify)..................4 & \\
\hline \multirow{15}{*}{9} & $\begin{array}{l}\text { Has a doctor or nurse or health worker at a clinic or } \\
\text { hospital told you that you have any of the following } \\
\text { conditions (multiple response possible) }\end{array}$ & & \\
\hline & \multirow{2}{*}{ (a) High blood pressure } & Yes .......................................1 & \\
\hline & & No......................................... & \\
\hline & \multirow{2}{*}{ (b) Depression } & Yes ........................................1 & \\
\hline & & 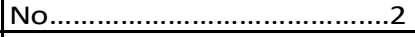 & \\
\hline & \multirow{2}{*}{ (c) Heart disease } & Yes ..........................................1 & \\
\hline & & 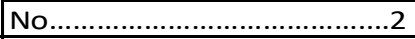 & \\
\hline & \multirow{2}{*}{ (d) Retinopathy (Eye disease) } & Yes ......................................... & \\
\hline & & 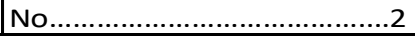 & \\
\hline & \multirow{2}{*}{ (e) Neuropathy (problem with the nerves) } & Yes ........................................1 & \\
\hline & & No......................................... & \\
\hline & \multirow{2}{*}{ (f) Kidney disease } & Yes ....................................... & \\
\hline & & No..................................... & \\
\hline & \multirow{2}{*}{ (g) Other } & Yes ................................. & \\
\hline & & 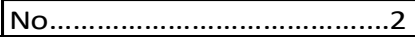 & \\
\hline \multirow{2}{*}{10} & Has a doctor or nurse or health worker at a clinic or & Yes ......................................1 & \\
\hline & hospital told you that you have had a stroke & No....................................... & \\
\hline 11 & What is vour sex & Male.......................................1 & \\
\hline & & 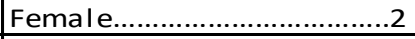 & \\
\hline & & African.................................1 & \\
\hline & & White..................................2 & \\
\hline 12 & What is your race & Coloured................................. & \\
\hline & & Indian/Asian......................4 & \\
\hline & & Other(specify).... & \\
\hline & & Single...................................1 & \\
\hline & & Married............................ & \\
\hline 13 & Please indicate your marital status & Divorced.................................. & \\
\hline & & Widowed...... & \\
\hline & & Co-habiting............................ & \\
\hline 14 & Has any close blood relative (father, mother, brother, sister & Yes .......................................1 & \\
\hline & or child) ever been told by a doctor or other health & No & \\
\hline & & 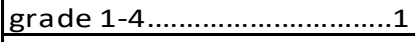 & \\
\hline & & 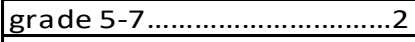 & \\
\hline 15 & Please indicate vour highest education level & 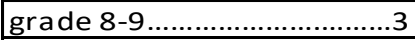 & \\
\hline 10 & 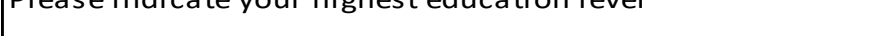 & 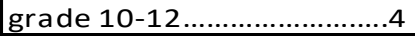 & \\
\hline & & College....... & \\
\hline & & 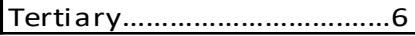 & \\
\hline
\end{tabular}

\section{INSTRUCTION TO INTERVIEWER - USE THE QUESTIONS BELOW TO GUIDE YOUR DISCUSSION WITH PATIENT (RECORD INTERVIEW)}




\section{Qualitative interview guide}

1. What was your first response when the doctor told you that you had diabetes?

Probing questions

- Diabetes knowledge prior to diagnosis

- What do you think caused the diabetes - lifestyle, family history, other beliefs

2. Can you tell me about your experiences with diabetes care at this facility?

Probing questions

- How do you feel about the information provided by doctors and nurses?

- How do you feel about the service you receive?

3. Overall what is your experience with managing your diabetes since you were diagnosed?

Probing questions

- How has diabetes affected your day to day activities?

- How confident are you with managing your diabetes?

4. How does your family and friends view your diabetes?

Probing questions

- What sort of support do you receive from them?

- What sort of support would you like them to give you?

5. What are your experiences engaging in the recommended diabetic self-care practices?

Probing questions

- How did your life change since you received your diabetes diagnosis?

- What was the biggest change?

- What did you find was the easiest change?

Medication adherence

- What purpose does your medication serve?

- What are your general feelings towards your diabetic medication?

- What are your experiences sticking to medication intake?

- What is your understanding of your medication regimen? Exercise recommendations

- What your experiences regarding the usefulness of exercising

Diet recommendations

- What are your experiences with following your diet recommendations? 
- Can you tell me more about the problems you face with sticking to diet recommendation?

- How would you describe your eating habits over the last seven days in comparison to diet recommendations?

6. Is there something else you would like to add?

7. Do you have any questions? 


\section{Appendix I: COREQ (COnsolidated criteria for REporting Qualitative research) Checklist}

Related to Chapter 6

\begin{tabular}{|c|c|c|c|c|}
\hline Topic & $\begin{array}{l}\text { Item } \\
\text { No. }\end{array}$ & Guide Questions/Description & Response & $\begin{array}{l}\text { Reported on } \\
\text { Page No. } \\
\text { Paragraph (P) }\end{array}$ \\
\hline \multicolumn{5}{|l|}{$\begin{array}{l}\text { Domain 1: Research } \\
\text { team and reflexivity }\end{array}$} \\
\hline \multicolumn{5}{|l|}{$\begin{array}{l}\text { Personal } \\
\text { characteristics }\end{array}$} \\
\hline Interviewer/facilitator & 1 & $\begin{array}{l}\text { Which author/s conducted the interview or } \\
\text { focus group? }\end{array}$ & $\begin{array}{l}\mathrm{CM} \text { and research } \\
\text { assistants }\end{array}$ & $\begin{array}{l}\text { Data collection } \\
(\mathrm{P} 1 \& 2)\end{array}$ \\
\hline Credentials & 2 & $\begin{array}{l}\text { What were the researcher's credentials? } \\
\text { E.g. PhD, MD }\end{array}$ & CM & $\begin{array}{l}\text { Data collection } \\
(\mathrm{P} 2)\end{array}$ \\
\hline Occupation & 3 & $\begin{array}{l}\text { What was their occupation at the time of } \\
\text { the study? }\end{array}$ & Researcher assistants & $\begin{array}{l}\text { Data collection } \\
\text { (P1) \& Author } \\
\text { contributions }\end{array}$ \\
\hline Gender & 4 & Was the researcher male or female? & Female & - \\
\hline $\begin{array}{l}\text { Experience and } \\
\text { training }\end{array}$ & 5 & $\begin{array}{l}\text { What experience or training did the } \\
\text { researcher have? }\end{array}$ & $\begin{array}{l}\text { Researcher has } \\
\text { previous knowledge } \\
\text { and experience in } \\
\text { qualitative research }\end{array}$ & - \\
\hline \multicolumn{5}{|l|}{$\begin{array}{l}\text { Relationship with } \\
\text { participants }\end{array}$} \\
\hline $\begin{array}{l}\text { Relationship } \\
\text { established }\end{array}$ & 6 & $\begin{array}{l}\text { Was a relationship established prior to } \\
\text { study commencement? }\end{array}$ & Yes & $\begin{array}{l}\text { Settings and } \\
\text { participants }\end{array}$ \\
\hline $\begin{array}{l}\text { Participant } \\
\text { knowledge of the } \\
\text { interviewer }\end{array}$ & 7 & $\begin{array}{l}\text { What did the participants know about the } \\
\text { researcher? e.g. personal goals, reasons for } \\
\text { doing the research }\end{array}$ & & $\begin{array}{l}\text { Data collection } \\
\text { (P3) }\end{array}$ \\
\hline $\begin{array}{l}\text { Interviewer } \\
\text { characteristics }\end{array}$ & 8 & $\begin{array}{l}\text { What characteristics were reported about } \\
\text { the inter viewer/facilitator? e.g. Bias, } \\
\text { assumptions, reasons and interests in the } \\
\text { research topic }\end{array}$ & $\begin{array}{l}\text { No conflict of interest } \\
\text { or interviewer related } \\
\text { biases were identified }\end{array}$ & - \\
\hline \multicolumn{5}{|l|}{$\begin{array}{l}\text { Domain 2: Study } \\
\text { design }\end{array}$} \\
\hline \multicolumn{5}{|l|}{$\begin{array}{l}\text { Theoretical } \\
\text { framework }\end{array}$} \\
\hline $\begin{array}{l}\text { Methodological } \\
\text { orientation and } \\
\text { Theory }\end{array}$ & 9 & $\begin{array}{l}\text { What methodological orientation was } \\
\text { stated to underpin the study? e.g. } \\
\text { grounded theory, discourse analysis, } \\
\text { ethnography, phenomenology, content } \\
\text { analysis }\end{array}$ & & $\begin{array}{l}\text { Data analysis } \\
\text { (P1) }\end{array}$ \\
\hline \multicolumn{5}{|l|}{ Participant selection } \\
\hline Sampling & 10 & $\begin{array}{l}\text { How were participants selected? e.g. } \\
\text { purposive, convenience, consecutive, } \\
\text { snowball }\end{array}$ & & $\begin{array}{l}\text { Settings and } \\
\text { Participants }\end{array}$ \\
\hline Method of approach & 11 & $\begin{array}{l}\text { How were participants approached? e.g. } \\
\text { face-to-face, telephone, mail, email }\end{array}$ & & $\begin{array}{l}\text { Settings and } \\
\text { Participants }\end{array}$ \\
\hline
\end{tabular}




\begin{tabular}{|c|c|c|c|c|}
\hline Sample size & 12 & How many participants were in the study? & & $\begin{array}{l}\text { Results - } \\
\text { demographic } \\
\text { characteristics }\end{array}$ \\
\hline Non-participation & 13 & $\begin{array}{l}\text { How many people refused to participate or } \\
\text { dropped out? Reasons? }\end{array}$ & $\begin{array}{l}\text { Study was conducted } \\
\text { as part of a large } \\
\text { quantitative study. } \\
\text { Participants who } \\
\text { participated in the } \\
\text { qualitative study had } \\
\text { previously agreed to } \\
\text { be interviewed in the } \\
\text { quantitative study. }\end{array}$ & - \\
\hline \multicolumn{5}{|l|}{ Setting } \\
\hline $\begin{array}{l}\text { Setting of data } \\
\text { collection }\end{array}$ & 14 & $\begin{array}{l}\text { Where was the data collected? e.g. home, } \\
\text { clinic, workplace }\end{array}$ & & $\begin{array}{l}\text { Settings and } \\
\text { Participants }\end{array}$ \\
\hline $\begin{array}{l}\text { Presence of } \\
\text { nonparticipants }\end{array}$ & 15 & $\begin{array}{l}\text { Was anyone else present besides the } \\
\text { participants and researchers? }\end{array}$ & & $\begin{array}{l}\text { Data collection } \\
\text { (P1\&2) }\end{array}$ \\
\hline Description of sample & 16 & $\begin{array}{l}\text { What are the important characteristics of } \\
\text { the sample? e.g. demographic data, date }\end{array}$ & & $\begin{array}{l}\text { Results - } \\
\text { demographic } \\
\text { characteristics }\end{array}$ \\
\hline \multicolumn{5}{|l|}{ Data collection } \\
\hline Interview guide & 17 & $\begin{array}{l}\text { Were questions, prompts, guides provided } \\
\text { by the authors? Was it pilot tested? }\end{array}$ & & Assessment tool \\
\hline Repeat interviews & 18 & $\begin{array}{l}\text { Were repeat inter views carried out? If yes, } \\
\text { how many? }\end{array}$ & No & - \\
\hline $\begin{array}{l}\text { Audio/visual } \\
\text { recording }\end{array}$ & 19 & $\begin{array}{l}\text { Did the research use audio or visual } \\
\text { recording to collect the data? }\end{array}$ & Yes & $\begin{array}{l}\text { Data collection } \\
\text { (P3) }\end{array}$ \\
\hline Field notes & 20 & $\begin{array}{l}\text { Were field notes made during and/or after } \\
\text { the interview or focus group? }\end{array}$ & $\begin{array}{l}\text { No additional field } \\
\text { notes }\end{array}$ & - \\
\hline Duration & 21 & $\begin{array}{l}\text { What was the duration of the inter views } \\
\text { or focus group? }\end{array}$ & & - \\
\hline Data saturation & 22 & Was data saturation discussed? & & $\begin{array}{l}\text { Data collection } \\
\text { (P3) }\end{array}$ \\
\hline Transcripts returned & 23 & $\begin{array}{l}\text { Were transcripts returned to participants } \\
\text { for comment and/or }\end{array}$ & No & - \\
\hline \multirow[t]{2}{*}{ Topic } & $\begin{array}{l}\text { Item } \\
\text { No. }\end{array}$ & Guide Questions/Description & & $\begin{array}{l}\text { Reported on } \\
\text { Page No. }\end{array}$ \\
\hline & & correction? & & \\
\hline \multicolumn{5}{|l|}{$\begin{array}{l}\text { Domain 3: analysis } \\
\text { and findings }\end{array}$} \\
\hline \multicolumn{5}{|l|}{ Data analysis } \\
\hline $\begin{array}{l}\text { Number of data } \\
\text { coders }\end{array}$ & 24 & How many data coders coded the data? & & $\begin{array}{l}\text { Data analysis } \\
\text { (P1) }\end{array}$ \\
\hline $\begin{array}{l}\text { Description of the } \\
\text { coding tree }\end{array}$ & 25 & $\begin{array}{l}\text { Did authors provide a description of the } \\
\text { coding tree? }\end{array}$ & & $\begin{array}{l}\text { Data analysis } \\
\text { (P1) }\end{array}$ \\
\hline Derivation of themes & 26 & $\begin{array}{l}\text { Were themes identified in advance or } \\
\text { derived from the data? }\end{array}$ & & $\begin{array}{l}\text { Data analysis } \\
\text { (P1) }\end{array}$ \\
\hline
\end{tabular}




\begin{tabular}{|c|c|c|c|c|}
\hline Software & 27 & $\begin{array}{l}\text { What software, if applicable, was used to } \\
\text { manage the data? }\end{array}$ & & $\begin{array}{l}\text { Data analysis } \\
\text { (P1) }\end{array}$ \\
\hline Participant checking & 28 & $\begin{array}{l}\text { Did participants provide feedback on the } \\
\text { findings? }\end{array}$ & No & - \\
\hline \multicolumn{5}{|l|}{ Reporting } \\
\hline Quotations presented & 29 & $\begin{array}{l}\text { Were participant quotations presented to } \\
\text { illustrate the themes/findings? } \\
\text { Was each quotation identified? e.g. } \\
\text { participant number }\end{array}$ & & Results \\
\hline $\begin{array}{l}\text { Data and findings } \\
\text { consistent }\end{array}$ & 30 & $\begin{array}{l}\text { Was there consistency between the data } \\
\text { presented and the findings? }\end{array}$ & & Results \\
\hline $\begin{array}{l}\text { Clarity of major } \\
\text { themes }\end{array}$ & 31 & $\begin{array}{l}\text { Were major themes clearly presented in } \\
\text { the findings? }\end{array}$ & & Results \\
\hline $\begin{array}{l}\text { Clarity of minor } \\
\text { themes }\end{array}$ & 32 & $\begin{array}{l}\text { Is there a description of diverse cases or } \\
\text { discussion of minor themes? }\end{array}$ & No & - \\
\hline
\end{tabular}


Dissertation Summary 


\section{Economic impact and inequalities in diabetes in South Africa - Summary}

The overall goal of this dissertation is to evaluate the economic impact and the inequality aspects of diabetes in South Africa. Diabetes is fast becoming a world pandemic, with global estimates indicating an increase in prevalence from 463 million in 2019 to 700 million in 2045. It is expected that the increase in the prevalence of diabetes within low- and middle-income countries (LMICs) will be faster when compared to high income countries. In LMICs diabetes was previously referred to as a disease of the affluent, being more concentrated amongst the rich, but evidence now suggests that it is more frequently being recorded amongst low socio-economic groups. The disease is one of the leading causes of mortality globally. In South Africa was recorded as the second biggest underlying cause of death in 2016. What makes diabetes an even bigger problem in LMICs, such as South Africa, is that it affects mostly the working age populations. This has the potential to affect the livelihood of families, productivity and economic growth. Besides, more than half of the people with diabetes are not diagnosed. Late diagnosis can be fatal and causes complications, which further lead to an increase in diabetes health care costs. Thus, diabetes is a chronic illness that is better managed when diagnosed early. The management of the disease also improves with self-care. However, in many LMICs, patients fail to adhere to recommended self-care practices due to the unavailability of resources.

The aim of this dissertation is to evaluate the economic impact and the inequality aspects of diabetes in South Africa. Given the anticipated increases in the prevalence of diabetes, a study of this nature is important in South Africa for various reasons. South Africa is reported to have one of the highest income inequalities in the world. These inequalities are intertwined with health and could drive the uneven distribution of diabetes and its health outcomes. Furthermore, the anticipated rise in the prevalence of diabetes affects the working age populations and could impact on the economic growth and the livelihood of families. The South African public health care system is already burdened by various challenges, an upsurge in demand for health care services by people with diabetes, will place pressure on already strained public health care resources. 


\section{Chapter 1}

In Chapter 1, we provide an introduction to the dissertation. The chapter sheds light into the growing burden of diabetes, its prevalence, economic impact and self-care management practices. The chapter further sheds light onto the concept of health inequalities and in particular health inequalities in South Africa. In order to contextualize the economic impact of diabetes and diabetes management in South Africa, the chapter also provides a description of the country's socio-economic outlook and burden of disease. The challenges associated with the diabetes costs and its management in South African can be better understood in relation to the country's general health care system. Thus, the chapter also provides a description of the health care system in South Africa. The objectives of the dissertation and the subsequent chapters are also defined in Chapter 1.

Objective 1: To capture the evidence on the cost of diabetes in Africa, review the methods used to calculate costs and identify areas for future research

Given the chronic nature of diabetes and that diabetes management is characterized by frequent hospital visits, the first objective of this dissertation is to review the evidence on the overall direct and indirect costs of diabetes. The overall focus is on the question "what are the direct and indirect costs associated with diabetes in Africa and what methods have been applied in the calculation of these costs".

Objective 2: To determine the catastrophic health expenditure and impoverishment amongst people with diabetes in South Africa

Given the huge income inequalities in South Africa, the second objective is to investigate the incidence, socio-economic inequalities and determinants of catastrophic health expenditure and impoverishment amongst people with diabetes in South Africa. Data were collected at two public hospitals with out-patient diabetes clinics. The analysis provides an opportunity to establish whether the government has been successful in protecting people with diabetes at public facilities from financial hardships.

Objective 3: To estimate socio-economic inequalities in diabetes and to examine the contribution of lifestyle factors to diabetes inequalities in South Africa.

Previous research has revealed the existence of inequalities in the prevalence of diabetes in South Africa. Therefore, the third objective investigates the socio-economic inequalities in 
diabetes in South Africa. For this we use both data on undiagnosed diabetes and self-reported data on a previous diabetes diagnosis. Given the role of modifiable lifestyle risk factors, the investigation also establishes the contribution of lifestyle factors to the prevalence of diabetes in South Africa.

Objective 4: To determine the inequalities in diabetes self-care practices and the determinants of diabetes self-care practices in South Africa

Evidence on the burden of diabetes would be incomplete without considering how individuals cope with managing the chronic illness. Given this, the inequalities and factors associated with diabetes self-care practices amongst people with diabetes are explored. Quantitative data gathered among patients attending two public diabetes health care clinics are used. In order to address the objectives of this study, findings from the quantitative data are complemented by findings from qualitative data on patients' experiences with diabetes health management.

Objective 5: To investigate the self-reported prevalence of and factors associated with diabetes and cardiovascular comorbidity in South Africa

Diabetes self-care is an essential element to prevent or delay the occurrence of diabetes complications, as indicated by the International Diabetes Federation (IDF). The presence of cardiovascular disease (CVD) in patients with diabetes leads to a reduction in the quality of life and an increase in health care related costs. Hence, after an assessment of self-care management practices amongst people with diabetes, we establish the prevalence of and factors associated with diabetes and CVD comorbidities.

\section{Chapter 2}

In this chapter, we provide a systematic review of the literature on the costs of diabetes mellitus in Africa. The chapter also reviews the methods used to estimate costs related to diabetes health care. The literature review is guided by the Preferred Reporting Items for Systematic Reviews (PRISMA) guidelines. A desk research was conducted in various databases including Pubmed, Medline, Science direct and Google Scholar. Keywords were carefully selected and checked by an experienced university librarian. The following eligibility criteria were used: peer reviewed English articles published between 2006 and 2016, that include at least one African country, are published in peer review journals and report original research findings on the cost of diabetes. 
Twenty-six studies were identified that met the inclusion criteria. The data extracted from the studies revealed that outpatient costs varied by study design, data source, perspective and health care cost categories included in the cost estimation. The studies that reported both drug costs and total costs, showed that drug costs took up a significant portion of total costs. Our review finds that the highest burden of diabetes health care costs is amongst those within the lowest socio-economic groups. Overall, the review reveals that there is a paucity of research on the cost of diabetes in African countries. The chapter concludes that given this gap in research, there is a need for further research aimed at providing evidence on diabetes costs.

\section{Chapter 3}

An analysis of the incidence, socio-economic inequalities and determinants of catastrophic health expenditure and impoverishment for diabetes care is provided in Chapter 3 . The study uses crosssectional data collected in 2017 from 396 patients visiting two public hospitals in Tshwane, Gauteng, that operate diabetes clinics. Face-to-face interviews were conducted with the patients using a structured questionnaire. Health care costs and transport costs were classified as catastrophic if they exceeded the $10 \%$ threshold of household capacity to pay (WHO method) or if they exceeded a variable threshold of the total household expenditure (Ataguba method). The study uses Erreygers concentration indices to estimate socio-economic inequalities and multivariable regression to identify the determinants of catastrophic health expenditure and impoverishment. Results from the study show that the incidence of catastrophic health expenditure and impoverishment vary with the methods used to estimate catastrophic health expenditure. Inequalities in catastrophic health expenditure favoured those in higher socioeconomic groups. The factors associated with catastrophic health expenditure and impoverishment vary with the method used in catastrophic health expenditure estimation. Overall the study reveals that financial protection for people with diabetes in public hospitals is limited. The chapter concludes that financing interventions must target the poor and there is a need for targeted interventions to improve access to health care.

\section{Chapter 4}

In Chapter 4, employing data from the 2012 South African National Health and Nutrition Examination Survey (SANHANES-1), concentration indices and the Wagstaff decomposition are 
used to estimate inequalities in diabetes and to determine the contribution of lifestyle factors to these inequalities. Previous studies that have estimated socio-economic inequalities in diabetes, made use of self-reported data. The IDF however reports that the majority of the people who are living with diabetes are not aware that they are, thus, our study estimates diabetes inequalities by using both diabetes self-reported data and data on undiagnosed diabetes. Our study shows the presence of pro-rich socio-economic inequalities in diabetes that are exacerbated by differences in dietary, lifestyle and metabolic factors. The decomposition shows that dietary, lifestyle and metabolic factors such as obesity and physical activity contribute to inequalities in diabetes. Although in comparison to other determinants, the contribution of these factors is modest, their contributions are important in the development of policies that address socioeconomic inequalities in the prevalence of diabetes.

\section{Chapter 5}

Chapter 5 focuses on estimating the inequalities and the determinants of diabetes self-care practices. For this purpose, we use quantitative data collected from two diabetes clinics located at two public hospitals in Tshwane. Face-to-face interviews were conducted with 396 diabetes patients using a structured questionnaire. The study considers diabetes self-care practices of dietary diversity, medication adherence, physical activity, self-monitoring of blood glucose, avoiding smoking and limited alcohol consumption. Concentration indices are used to estimate socio-economic inequalities in self-care practices and regression analysis conducted to determine factors associated with self-care. Our results show that physical activity was the least adhered to self-care practice, followed by dietary diversity, medication adherence, not smoking, selfmonitoring of blood glucose and limited alcohol consumption. The presence of complications or additional illnesses may be linked to the high prevalence of non-adherence to physical activity. Adherence to dietary diversity and physical exercise was more concentrated amongst those in higher socio-economic groups whilst not smoking was concentrated amongst those in low socioeconomic groups. Our regression analysis shows that even after including the same variables, the association of each variable with each self-care behaviour differs. The findings suggest that efforts to improve self-care must address socio-economic inequalities. Furthermore, the strategies used to address each self-care behaviour should be behaviour-specific given the different determinants. 


\section{Chapter 6}

In order to capture the experiences of diabetes self-care management practices amongst people with diabetes, we complemented the findings from the previous chapter with a qualitative study. The key objective is to explore the challenges people with diabetes face in diabetes self-care and the factors that enable or inhibit them from adhering to recommended diabetes self-care practices. Using an interview guide with open ended questions, 28 purposively sampled patients visiting two public diabetes clinics and one private diabetes clinic were interviewed. Transcribed interviews were entered into Atlas.ti and data analysis was conducted using content analysis and guided by the Theory of Triadic Influence. The results from the analysis are presented for the intra-personal, inter-personal and environmental streams. The results reveal enablers and inhibitors of diabetes emanating from all three streams of influence. Intra-personal factors such as diabetes knowledge and depression were identified as enabler and inhibitor of diabetes selfcare respectively. Inter-personal factors such as supportive interactions with family and nonsupportive interactions with family were identified as enabler and inhibitor of diabetes self-care respectively. Environmental factors such as the use of technology devices and financial difficulty were identified as enabler and inhibitor of diabetes self-care respectively. The study demonstrates the importance of multi-level approaches to promoting diabetes self-care.

\section{Chapter 7}

People with diabetes are at an increased risk of developing CVD when compared to people without diabetes. However, no study has investigated CVDs in people with diabetes in South Africa. Therefore, using data from the 2012 SANHANES-1, Chapter 7 examines the self-reported prevalence of and factors associated with diabetes and diabetes CVD comorbidity in South Africa. Our findings show that indeed the majority of people with diabetes in our study, self-reported having at least one additional CVD. The results from the regression analysis suggested that after applying a common set of variables, the statistical significance of the effect of each determinant on diabetes or diabetes CVD comorbidity was different. Even when variables were associated with both diabetes and diabetes CVD comorbidity, the magnitude of the effect differed. We find for example that older age was associated with both the presence of diabetes and the presence of diabetes CVD comorbidity. The magnitude of the coefficients was slightly larger for diabetes CVD when compared to diabetes only. This indicates a slightly higher likelihood of association. 
Other differences are for example that being Indian was associated with having diabetes but not associated with diabetes CVD comorbidity, high income was associated with diabetes CVD comorbidity but not with diabetes. These findings give insight into the factors associated with having diabetes only and having diabetes and CVD comorbidity. The results can aid decision making for improved diabetes outcomes.

\section{Chapter 8}

Finally, Chapter 8 summarises and discusses the main findings from the studies conducted in the dissertation. As discussed in the chapter, in South Africa, differences in socio-economic inequalities exist in terms of diabetes prevalence, diabetes health care costs and diabetes selfcare practices. It is reported that high income inequalities are intertwined with high health inequalities. South Africa is reported to have one of the highest income inequalities in the world and we see this translated into inequalities in various aspects of diabetes. By using concentration indices, we find that the direction of inequality varies for diabetes prevalence, diabetes health care costs and each diabetes self-care practice. Efforts to address these inequalities should focus on addressing income inequalities within the country. Our findings on the inequalities in the economic impact of diabetes call for targeted policies that protect people with diabetes within low socio-economic groups from catastrophic health expenditure.

We also show that financial difficulty amongst people with diabetes in South Africa is both an outcome of self-care and a barrier to some diabetes self-care practices. Our literature review in Chapter 2 shows that the economic burden of diabetes is huge. This is consistent with findings from Chapter 3, which show that the fees for using health care may lead to financial difficulty for some patients as reflected in the estimates of catastrophic health expenditure and impoverishment. Chapter 6 also goes on to show that individuals with diabetes may fail to adhere to self-care practices such as healthy diet due to financial difficulties. The findings from our study call for the elimination of user fees for people with diabetes accessing public hospitals and for the consideration of the financial challenges that patients face during the tailoring of individualised patient care. 
Evidence in South Africa suggests that the factors that influence health care behaviour, are varied and originate from multiple levels. Given that the determinants of self-care and the magnitude of their effect is not consistent across the self-care behaviour, interventions that aim to improve diabetes self-care practice should take into consideration the health behaviour to be addressed and the context in which the behaviour occurs. Given that multi-level factors influence the occurrence of diabetes self-care, programmes that promote self-care need to be comprehensive and target all levels of influence. Due to the multifaceted and multivariable nature of diabetes and its link to other diseases, we recommend a holistic approach to diabetes management that also takes into consideration the various social aspects linked to diabetes. 


\section{Valorisation Addendum}

Diabetes is a major threat to global health that affects one in eleven adults between the ages of 20 to 79 years. Global estimates indicate an increase in prevalence from 463 million in 2019 to 700 million in 2045. It is expected that the increase in the prevalence of diabetes within low- and middle-income countries will be faster when compared to high income countries. Diabetes is also a major threat to economic activity as it mostly affects the working age population ( 3 out of 4 people with diabetes). Diabetes is also one of the leading causes of mortality globally. In South Africa it was recorded as the second biggest underlying cause of death in 2016. Without strong, targeted, evidence-based interventions diabetes has the potential to consume huge health budgets and overwhelm the country's health care system.

The South African government's Strategic Plan for the Prevention and Control of NCDs in South Africa provides direction for reducing the morbidity and mortality from non-communicable diseases, such as diabetes. As set out in the plan, one of the three primary approaches identified to minimise the burden of NCDs, such as diabetes, is the expansion of monitoring, surveillance and research of NCDs and their risk factors (National Department of Health, 2013).

This dissertation builds on the above by providing knowledge and insight that may influence policy or interventions targeted at the prevention and control of diabetes and its health outcomes. The overall goal of the dissertation was to evaluate the economic impact and the inequality aspects of diabetes in South Africa. The empirical analyses of this dissertation are based on two datasets: (1) the 2012 South African National Health and Nutrition Examination Survey (SANHANES-1); (2) primary data collected from three diabetes health care clinics in Tshwane health district in the Gauteng Province.

Once scientific research is completed, the dissemination of its results becomes critical to the prevention and control of diabetes. To achieve the targets set out in the Sustainable Development Goals targeted for 2030, it is important for the research to reach various stakeholders such as fellow researchers, people living with diabetes, their families, health care professionals, and policy makers. The PhD regulations at Maastricht university in 2018 define the process of valorisation as "the process of creating value from knowledge, by making knowledge 
suitable and/or available for social (and/or economic) use and by making knowledge suitable for translation into competitive products, services, processes and new commercial activities". Hence, the purpose of this chapter is to provide an overview of the value of the results presented in this dissertation and how they can be put into practice.

\section{Relevance for researchers}

To promote further research, the results from this dissertation must be made available to fellow researchers and practitioners. To allow for this, five out of the six articles described in this dissertation have been published and the remaining one is currently under review. The five published articles are available in open access journals. In addition to stimulating debate, gathering additional information and obtaining additional insights, the results from some of the studies were presented at various conferences such as the African Health Economics Association conference in Ghana, the Public Health Association of South Africa conference and the Human Sciences Research Council conference in South Africa, before publication.

The studies described in this dissertation are relevant for researchers in the field of diabetes, cost of illness and socio-economic inequalities in health. Whilst the entire dissertation is relevant for all researchers interested in diabetes, Chapters 2 and 3 focus on cost of illness and Chapters 3, 4 and 5 assess the various inequality aspects of diabetes. The systematic review presented in Chapter 2 makes researchers aware of the shortcomings of cost of illness studies but also highlights their important role in conducting full economic evaluations of treatments and other health care interventions. Our results from Chapter 3 also point out to researchers the shortcomings of the various methods used within literature to measure catastrophic health expenditure and impoverishment. For robustness, our study employs two different methods in the estimation of catastrophic health expenditure and impoverishment. The study also applies various thresholds in the estimation of catastrophic health expenditure, leaving it to the reader to determine their selection. Whilst providing an overview of the economic impact of diabetes and inequality aspects in diabetes, the dissertation provides knowledge gaps in each chapter. This may guide future researchers to close these gaps in research. In addition to its contribution to the current scientific knowledge on the topic, the research may provide a reference case for future studies fellow researchers might conduct. 


\section{Relevance for people living with diabetes, people without diabetes, and health care professionals}

The studies presented in this dissertation are relevant to people living with diabetes and people without diabetes as well as health care professionals. Whilst diabetes self-care is mostly the patient's responsibility (Funnell \& Anderson, 2000), it is well established that health care professionals play a role in supporting diabetes self-care by patients and ultimately, in improving clinical outcomes. Healthcare professionals have the role of providing diabetic education and advice regarding self-care practices. There is increasing recognition that there is need for health care professionals to facilitate patient empowerment by focusing on the understanding the patient's diabetes experiences and treatment goals. An identification of the challenges that patients face in diabetes self-care (Chapter 5 and Chapter 6), presupposes health-care professional and patient interactions to be collaborative rather than directive. Such interactions encourage patient involvement in the care process, and promotes shared decision making.

The studies presented in this dissertation are also relevant for people living with diabetes and people without diabetes. Although it may be unrealistic to expect everyone to understand the studies presented in this dissertation completely the conclusions have been presented in a comprehensible way. It is important for both people living with diabetes and people without diabetes to be aware of: (1) the role that lifestyle factors play in the progression of socioeconomic inequalities in both the onset and progression of diabetes (Chapter 4 and Chapter 5); (2) the factors that influence diabetes cardio-vascular disease comorbidity (Chapter 7); and (3) the economic implications of diabetes (Chapter 3). Such awareness may influence actions that prevent the development of diabetes or diabetes related complications. Being acquainted with diabetes and its challenges may mean family members are better equipped to participate in diabetes management. In the long run, people living with diabetes and people without diabetes do benefit from the policy implications of the scientific research reported in this dissertation.

\section{Relevance of policy makers}

For policy makers, the dissertation provides information on the economic impact (Chapter 3 ) and inequality aspects of diabetes (Chapters 4 to 7 ) in South Africa. The systematic review provided in Chapter 2 highlights to policy makers the huge economic impact (direct and indirect) of 
diabetes in African countries. The review may inspire and give a message regarding the importance of addressing such costs, which impact economies, health care systems, societies, and individuals. Evidence generated from this dissertation provides policy implications for the renewed commitment to ensuring that the health care system offers financial protection from risks of catastrophic expenditure or impoverishment due to diabetes related health care payments. The dissertation suggests that financial protection of people with diabetes in public hospitals is limited highlighting the weaknesses of the current uniform fee payment schedule. This finding points to the need to eliminate user fees for diabetes patients who access public hospitals. This is particularly important for the achievement of universal health coverage in South Africa.

In addition to facing costs related to accessing healthcare, people with diabetes are faced with financial difficulties in adhering to some self -care practices. These challenges are discussed in Chapter 5 of the dissertation. The results suggest that individuals are often faced with financial difficulties in adhering to diet recommendations, medication, and glucose monitoring costs. Whilst pointing to the economic challenges faced by many individuals these challenges also highlight the need for a National Health Insurance Scheme that ensures distribution of chronic medication and glucose monitoring kits for people with diabetes.

The huge income inequalities in South Africa are intertwined with inequalities in health. Persisting social disparities, poverty and inequality in socio-economic status continue to influence the disproportionate distribution of social determinants of health and ultimately exacerbate inequalities in health and well-being (Ataguba et al., 2011). The dissertation suggests that in South Africa, differences in socio-economic inequalities exist in terms of diabetes prevalence, diabetes health care costs and diabetes self-care practices. We also find that socio-economic status has a role to play in the distribution of diabetes cardio-vascular disease comorbidity. These findings are important to policy makers in terms of informing the design of effective strategies and policies for addressing income inequalities and encouraging healthy lifestyles.

In the fight against diabetes South Africa could also draw lessons from the huge HIV programme within the country. Adherence clubs, community level programmes, facility-based interventions 
and the introduction of case managers may be useful strategies for the promotion of healthy lifestyles, which are essential to the prevention and control of diabetes. 


\section{Word of thanks}

As my PhD journey comes to an end, I cannot help but be filed with a sense of immense gratitude to those who have walked this journey with me. I am very grateful to God for the countless blessings and strength that allowed me to complete this research. An overwhelming sense of gratitude towards my supervisors, Prof Wim Groot and Prof Milena Pavlova, without whom this dissertation would not have been possible. I thank you for your commitment, patience, and guidance throughout this journey. In the many occasions in which I felt uncertain, you always made things clearer. Your easy to approach personas made this journey manageable. Under your guidance I have transformed from being a perplexed, inexperienced researcher to be the author of several research publications. I feel extremely blessed to have worked with you. You were such excellent supervisors and I learnt a lot through your guidance. Also, thanks to Suus for all the administrative support and organising our meetings.

My utmost gratitude also goes to Prof Charles Hongoro whom I met during my PhD internship at the Human Sciences Research Council. During the first few months of this journey, I was often overwhelmed by stressful situations and anxiety. Somehow you always managed to bring me back to focus. You have been a big part of my life during this period and your open-door policy helped make this journey manageable and enjoyable. On several occasions I remember texting or calling you when in need of help, even during non-work hours and weekends. You are a book of knowledge and many of your words will continue to ring in my head as I continue my work in research.

There are several people within the HSRC who helped me during my PhD journey. Many provided input and advice in my proposal development, funding applications, data analysis and in the review of my work. Your words of advice and encouragement are greatly appreciated. My gratitude goes to Dr Lumbwe Chola. You are what I would refer to as my 'day ONE'. You were there right from the beginning when this $\mathrm{PhD}$ was still an idea. Even after your departure from the HSRC you remained a valuable part of my journey. My gratitude also goes to Prof Booysen for co-co-authoring with us. I am forever grateful for your support and providing so much detailed guidance. To Tanja and Nozuko, I thoroughly enjoyed working with you and I am forever grateful for your support and friendship. My gratitude also goes to Benelton. Thank you for the 
administrative support you provided at the HSRC. Without your commitment this journey would have been difficult.

I would also like to thank the reviewing committee. I greatly appreciate the time you took to review my work. Your feedback and comments helped improve the final dissertation. I am also so very grateful to the Study Fund for South Africans that funded my yearly travels to the Netherlands. Thank you for making this possible.

To the friends I made during my visits to Maastricht University, this journey would not have been exciting without you. To Tatenda, thank you for welcoming me to Maastricht on each of my visits and ensuring I settled in well on each occasion. Our trips within Europe will always be one of the most memorable parts of my journey.

I am humbled by the number of family and friends who have been critical to the completion of my PhD and who have made this journey exciting. I am grateful to my parents and sisters. To my sister Nyarai, who saw value in this research right from the beginning. Having been diagnosed with diabetes more than 15 years ago you were my inspiration for this research. To my dad, Elliot Mutyambizi and little sister, Tendai who was diagnosed with diabetes during the course of my PhD you are both incredibly amazing. I always enjoyed the chats we had regarding my research and the insight you always brought to these chats. Thank you, dad for seeing the value in education and supporting me in my chosen field. To my mum, Amai, you have been a constant pillar of support right from the beginning. Thank you for enduring my numerous distraught, stressful phone calls, encouraging me and reassuring me that I can do this. To my beloved husband, Benjamin, who had to endure the seemingly never ending and constant stressful episodes of my PhD during its final year, thank you for the support and ensuring that during the hectic times I was fed and taken care of, you are heaven sent. To the rest of my family and friends, who are too many to mention, thank you so much for your role in this journey.

To God be the glory and honour. 


\section{Curriculum Vitae}

Chipo Mutyambizi was born on 21 February 1985. After graduating from high school in 2003, she enrolled at the University of Zimbabwe and began her Bachelor's degree in Economics in 2004. She finished her Bachelor's degree in August 2007 and joined Stanbic Bank Zimbabwe immediately after graduating. In 2009, she left Stanbic Bank and moved to Stellenbosch University in South Africa where she did both her Honours and Master's degree in Economics. Whilst at Stellenbosch University, she worked as a tutor for the undergraduate programme at the department of Economics. Upon completion of her Masters, she joined Venn Diagnostics as a Utility Cost Analyst. In parallel to this, she worked as a research assistant, on the Western Cape Government's Municipal Economic Review and Outlook yearly report from 2012 - 2015. She also took part in various ad-hoc research projects under the leadership of her Masters' thesis supervisor, Professor Philip Black. She then joined the Human Sciences Research Council (HSRC) as a PhD Intern in 2016 in the Population Health, Health Systems and Innovation Unit (PHHSI) where she has been involved in various research projects. In the same year, she became a PhD Student at the School of Public Health and Primary Care of the Faculty of Health, Medicine and Life Sciences at the department of Health Services Research at Maastricht University. In March 2019 she moved to the Research Use and Impact Assessment Unit, still within the HSRC, where she continued taking part in research projects such as the Study on Global Aging and Adult Health Wave 3 (SAGE). In November 2019, Chipo was the recipient of the HSRC junior research excellence award. On the $1^{\text {st }}$ of January 2020 she left the HSRC and joined Anova Health Institute to work as a Public Health Analyst. 


\section{List of publications and presentation}

\section{Articles - peer reviewed journals}

- Mutyambizi C, Pavlova M, Hongoro C, Groot W. Inequalities and factors associated with adherence to diabetes self-care practices amongst patients at two public hospitals in Gauteng, South Africa. BMC Endocrine Disorders. 2020;20(1):15

- Mutyambizi C, Booysen F, Stornes P, Eikemo T.A. Subjective social status and inequalities in depressive symptoms: a gender specific decomposition analysis for South Africa. International journal for equity in health. 2019

- Mutyambizi C, Pavlova M, Hongoro C, Booysen F, Groot W. Incidence, socio-economic inequalities and determinants of catastrophic health expenditure and impoverishment for diabetes care in South Africa: a study at two public hospitals in Tshwane. International journal for equity in health. 2019;18(1):73.

- Mutyambizi C, Booysen F, Stoke A, Pavlova M, Groot W. Lifestyle and socio-economic inequalities in diabetes prevalence in South Africa: A decomposition analysis. PLOS ONE. 2019.

- Mutyambizi C, Pavlova M, Chola L, Hongoro C, Groot W. Cost of diabetes mellitus in Africa: a systematic review of existing literature. Globalization and health. 2018;14(1):3

- Mchiza ZJ, Parker Wa, Sewpaul R, Job N, Chola L, Mutyambizi C, et al. Understanding the determinants of hemoglobin and iron status: adolescent-adult women comparisons in SANHANES-1. Annals of the New York Academy of Sciences. 2018.

- Mutyambizi C, Chola L, Groot W, Pavlova M, Labadarios D, Hongoro C. The extent and determinants of diabetes and cardiovascular disease comorbidity in South Africa-results from the South African National Health and Nutrition Examination Survey (SANHANES-1). BMC Public Health. 2017;17(1):745.

- Chola, L. Mutyambizi, C. Sewpaul, R. Parker, W. Mchiza,Z. Labadarios, D. Hongoro, H. Selfreported diabetes during pregnancy in the South African National Health and Nutrition Examination Survey: extent and social determinants. BMC Pregnancy and Childbirth. 2017. 
Articles currently under peer - review

- Mutyambizi C, Mokhele T, Ndinda C, Hongoro C. Access to and satisfaction with basic services in informal settlements: results from a baseline assessment survey

- Mutyambizi C, Pavlova M, Hongoro C, Groot W. Barriers and enablers of diabetes self-care - a qualitative study of diabetic patients in South Africa. 


\section{Conference presentations}

\begin{tabular}{|c|c|c|c|}
\hline Presentation title & \begin{tabular}{|l|} 
Conference \\
\end{tabular} & Dates & Place \\
\hline \multirow{3}{*}{$\begin{array}{l}\text { Incidence, socio-economic } \\
\text { inequalities and determinants of } \\
\text { catastrophic health expenditure } \\
\text { and impoverishment for } \\
\text { diabetes care in South Africa: a } \\
\text { study at two public hospitals in } \\
\text { Tshwane. }\end{array}$} & $\begin{array}{l}\text { African Health } \\
\text { Economic and Policy } \\
\text { Association } \\
\text { Conferences }\end{array}$ & $\begin{array}{l}11-14 \text { March } \\
2019\end{array}$ & Accra, Ghana \\
\hline & $\begin{array}{l}\text { Human Science } \\
\text { Research Council } \\
\text { Conference }\end{array}$ & $\begin{array}{l}\text { 12-14 September } \\
2018\end{array}$ & $\begin{array}{l}\text { St George Hotel, } \\
\text { Pretoria }\end{array}$ \\
\hline & $\begin{array}{l}\text { Public Health } \\
\text { Association of South } \\
\text { Africa Conference } \\
\end{array}$ & $\begin{array}{l}10-12 \text { September } \\
2018\end{array}$ & Parys \\
\hline \multirow{3}{*}{$\begin{array}{l}\text { Inequalities in depression } \\
\text { symptoms due to subjective } \\
\text { social status: a decomposition } \\
\text { analysis }\end{array}$} & $\begin{array}{l}\text { African Health } \\
\text { Economic and Policy } \\
\text { Association } \\
\text { Conferences }\end{array}$ & $\begin{array}{l}11-14 \text { March } \\
2019\end{array}$ & Accra, Ghana \\
\hline & $\begin{array}{l}\text { Human Science } \\
\text { Research Council } \\
\text { Conference } \\
\end{array}$ & $\begin{array}{l}\text { 12-14 September } \\
2018\end{array}$ & $\begin{array}{l}\text { St George Hotel, } \\
\text { Pretoria }\end{array}$ \\
\hline & $\begin{array}{l}\text { Public Health } \\
\text { Association of South } \\
\text { Africa Conference } \\
\end{array}$ & $\begin{array}{l}10-12 \text { September } \\
2018\end{array}$ & Parys \\
\hline $\begin{array}{l}\text { Extent and determinants of } \\
\text { diabetes and cardiovascular } \\
\text { disease comorbidity in South } \\
\text { Africa - results from the South } \\
\text { African National Health and } \\
\text { Nutrition Examination Survey } \\
\text { (SANHANES 1)", }\end{array}$ & $\begin{array}{l}\text { Johannesburg } \\
\text { Health District } \\
\text { Research } \\
\text { Conference }\end{array}$ & $\begin{array}{l}17 \text { November } \\
2016\end{array}$ & Johannesburg \\
\hline $\begin{array}{l}\text { Out of Pocket health care } \\
\text { expenditure by diabetic } \\
\text { individuals in South Africa: } \\
\text { Results from the South African } \\
\text { Nutrition and Health } \\
\text { Examination Survey }\end{array}$ & $\begin{array}{l}\text { Human Science } \\
\text { Research Council } \\
\text { Conference }\end{array}$ & $\begin{array}{l}\text { 22-23 September } \\
2016\end{array}$ & $\begin{array}{l}\text { Indaba Hotel } \\
\text { Conference } \\
\text { Centre, } \\
\text { Johannesburg }\end{array}$ \\
\hline $\begin{array}{l}\text { Closing the Collateral Gap } \\
\text { among the Poor: A theoretical } \\
\text { Note }\end{array}$ & $\begin{array}{l}\text { Value } 2014 \\
\text { Conference }\end{array}$ & $\begin{array}{l}26 \text { to } 27 \text { May } \\
2014\end{array}$ & $\begin{array}{l}\text { Johannesburg, } \\
\text { South Africa }\end{array}$ \\
\hline
\end{tabular}


